\title{
The Influence of Slanted Ground Surfaces on Trunk Biomechanics and Stability During Trunk Bending
}

Boyi Hu

Follow this and additional works at: https://researchrepository.wvu.edu/etd

\section{Recommended Citation}

$\mathrm{Hu}, \mathrm{Boyi}$, "The Influence of Slanted Ground Surfaces on Trunk Biomechanics and Stability During Trunk Bending" (2016). Graduate Theses, Dissertations, and Problem Reports. 5828.

https://researchrepository.wvu.edu/etd/5828

This Dissertation is protected by copyright and/or related rights. It has been brought to you by the The Research Repository @ WVU with permission from the rights-holder(s). You are free to use this Dissertation in any way that is permitted by the copyright and related rights legislation that applies to your use. For other uses you must obtain permission from the rights-holder(s) directly, unless additional rights are indicated by a Creative Commons license in the record and/ or on the work itself. This Dissertation has been accepted for inclusion in WVU Graduate Theses, Dissertations, and Problem Reports collection by an authorized administrator of The Research Repository @ WVU.

For more information, please contact researchrepository@mail.wvu.edu. 
The Influence of Slanted Ground Surfaces on Trunk Biomechanics and Stability During Trunk Bending

Boyi Hu

Dissertation submitted to the Statler College of Engineering and Mineral Resources at West Virginia University in partial fulfillment of the requirements for the degree of DOCTOR OF PHILOSOPHY

\author{
Committee Members: \\ Xiaopeng Ning, Ph.D., Chair \\ Fei, Dai, Ph.D. \\ Majid, Jaridi, Ph.D. \\ Ashish, D. Nimbarte, Ph.D. \\ Feng, Yang, Ph.D.
}

Department of Industrial and Management System Engineering

Morgantown, West Virginia

2016

Keywords: Low Back Pain, Slanted Ground Surface, Lumbar Flexion and Extension, Flexion Relaxation Phenomenon, Spinal Stability

Copyright $(\mathcal{C}$ Boyi Hu, 2016. All rights reserved. 


\section{ABSTRACT \\ The Influence of Slanted Ground Surfaces on Trunk Biomechanics and Stability During Trunk Bending}

\section{Boyi Hu}

Previous studies have shown that working on uneven ground surfaces is linked to alternations of the lumbar active and passive tissue load sharing mechanism and reductions in spinal stability, which is highly associated with the risk of low back pain. The purpose of the current research was to investigate the changes of the lumbar flexion relaxation phenomenon and lumbar segmental stability while working on slanted ground surfaces. In experiment one, fourteen male participants performed sagittal plane, trunk flexion-extension tasks on three laterally slanted ground surfaces (flat ground, $15^{\circ}$ and $30^{\circ}$ ) while their lumbar muscle activities and trunk kinematic performances were recorded. Results showed that flexion relaxation occurred up to $6.2^{\circ}$ earlier among the ipsilateral lumbar muscles with an increase in laterally slanted ground angle; however, the contralateral side was not affected as much. The results of experiment one demonstrated that the uneven ground surface has the potential of changing the lumbar tissue load-sharing mechanism and affect lumbar biomechanical responses.

Based on the results of experiment one, a follow up experiment was conducted to further explore the effects of antero-posteriorly slanted ground surfaces and trunk asymmetry on spine biomechanics during trunk bending motion. More specifically, we investigated the influences of antero-posteriorly slanted ground surfaces, trunk asymmetry and their interaction on the lumbar extensor muscle flexion relaxation phenomenon during trunk flexion 
motion, as well as lumbar segmental stability performance in deep trunk bending postures. Fourteen healthy male participants performed sagittally symmetric and asymmetric trunk bending tasks on one flat and two antero-posteriorly slanted surfaces $\left(-15^{\circ}\right.$ (uphill facing) and $15^{\circ}$ (downhill facing)), while their lumbar muscle electromyography and trunk kinematics were recorded. Results showed that standing on a downhill facing slanted surface could delay the onset of lumbar muscle flexion relaxation phenomenon, while standing on uphill facing ground causes lumbar muscle flexion relaxation to occur earlier. During asymmetric trunk bending, flexion relaxation occurred earlier among the contralateral side of lumbar muscles; significantly smaller maximum lumbar flexion and trunk inclination angles were also observed.

Regarding lumbar segmental stability, our results demonstrated that the asymmetric condition significantly reduced lumbar segmental stability by enlarging C7, T12 and S1 segments' sway distance and sway velocity and these effects were more pronounced in the antero-posterial (AP) direction. Furthermore, results indicated AREA CE might be a more appropriate method to be applied to analyze lumbar segmental stability in the asymmetric conditions than other sway area methods (i.e. AREA CC, AREA SW, etc.).

In terms of lumbar segmental stability, results showed that uphill facing surface generated negative effects on lumbar segmental stability, and thus is the least desirable working condition. Downhill facing surface showed insignificant effects on lumbar segmental stability compared with the flat ground condition in most cases. Finally, when performing tasks on slanted surfaces with a deep bending posture, S1 segment demonstrated the worst segmental stability among all three segments tested in the current research. 
To summarize, our results confirmed that uneven ground surfaces and asymmetric working postures could negatively affect the trunk biomechanics performance, and thus possibly increase the risk of low back pain. Findings from the current research demonstrated the potential need of including ground working conditions to ergonomic risk assessment tools and biomechanical models. 


\section{TABLE OF CONTENTS}

LIST OF FIGURES ….........................................................................................iii

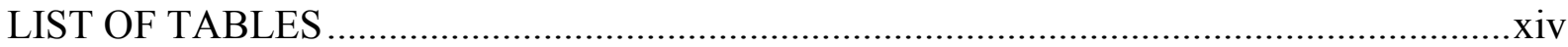

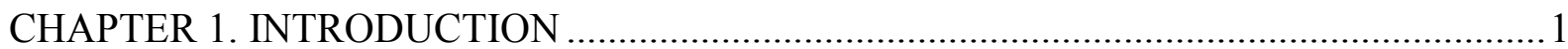

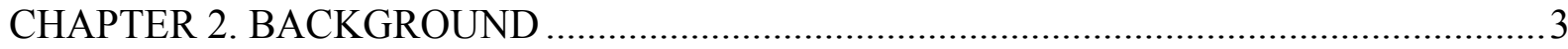

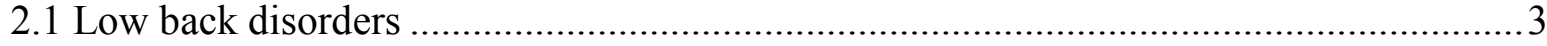

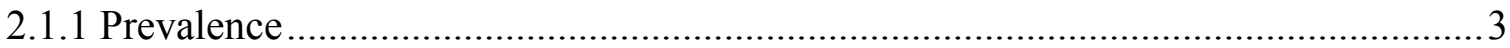

2.1.2 Health and economic impact....................................................................... 7

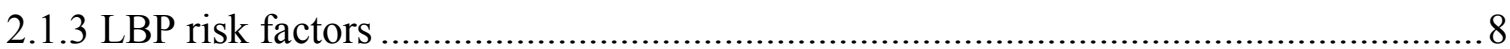

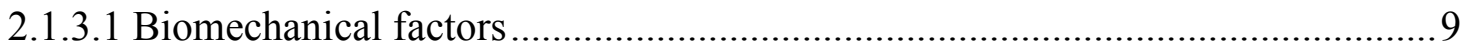

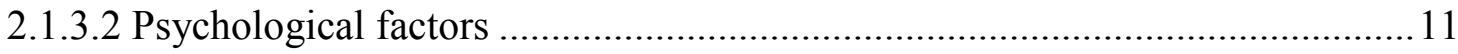

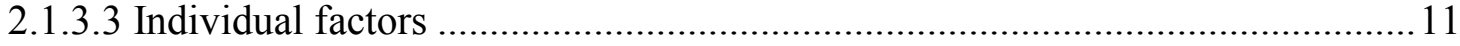

2.2 Uneven ground condition as an occupational injury risk factor................................ 12

2.2.1 Uneven ground in occupational environment ................................................. 12

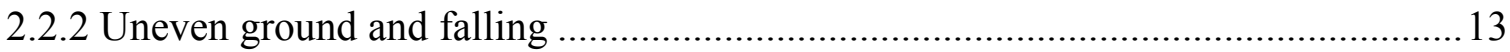

2.2.3 Uneven ground and musculoskeletal disorders ................................................. 16

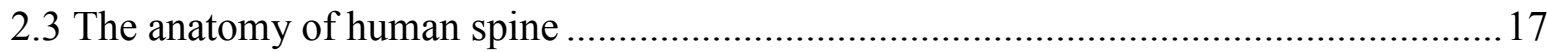

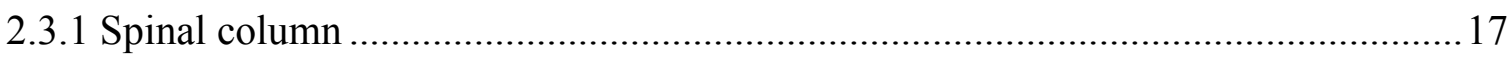

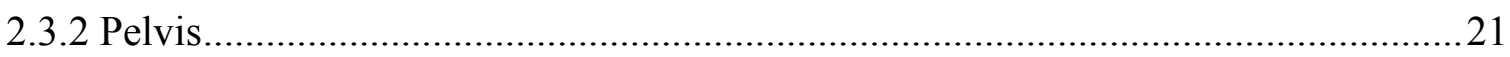

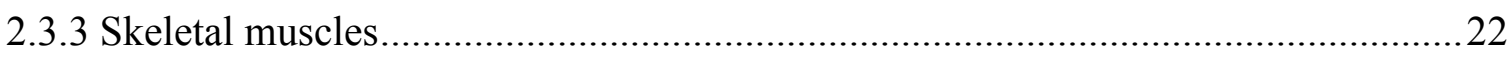

2.3.3.1 Lumbar muscles .................................................................................... 22

2.3.3.2 Abdominal muscles....................................................................... 24

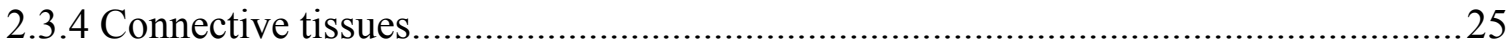

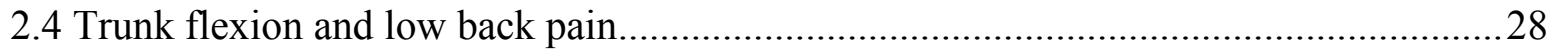

2.4.1 Trunk muscle concentric and eccentric exertions ..........................................28

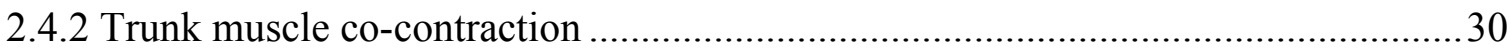

2.5 Lumbar muscle flexion-relaxation phenomenon................................................. 32

2.5.1 The underlying mechanism of lumbar muscle FRP......................................... 33

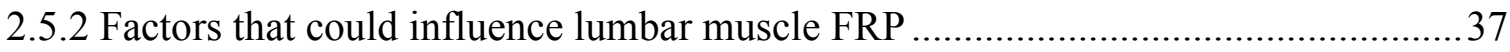

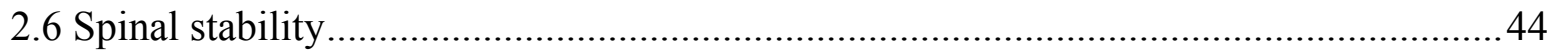




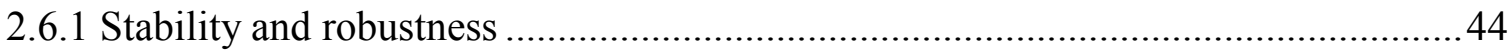

2.6.2 Feedback control in the spinal balance control system...........................................46

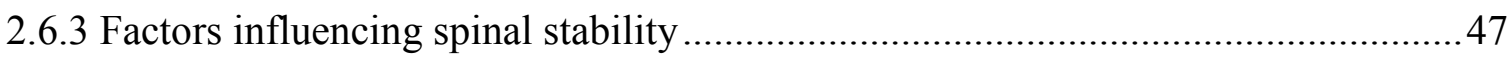

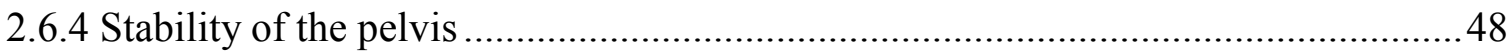

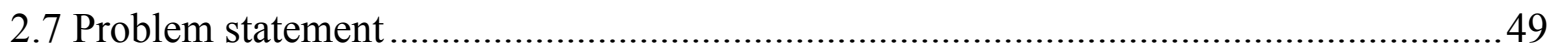

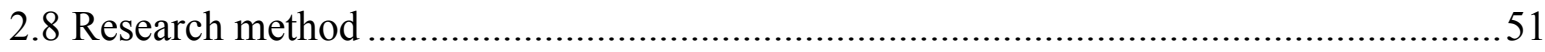

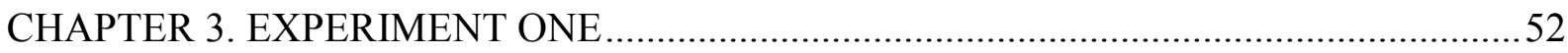

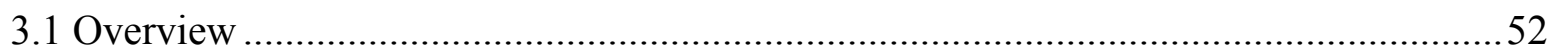

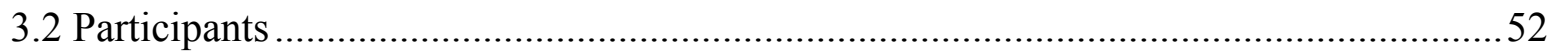

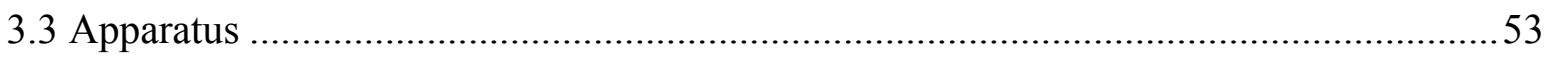

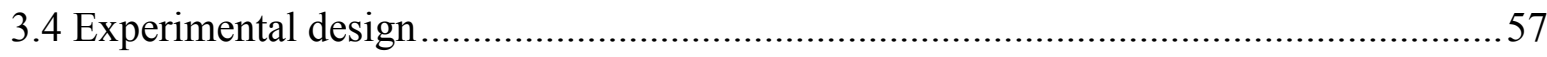

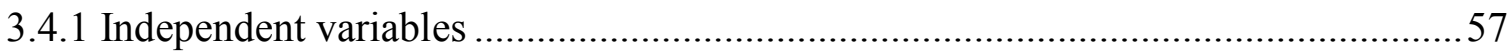

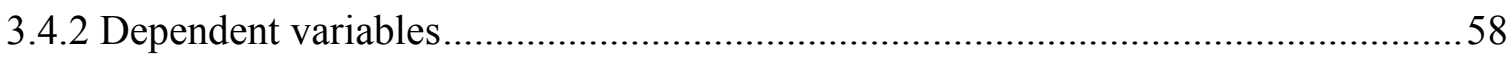

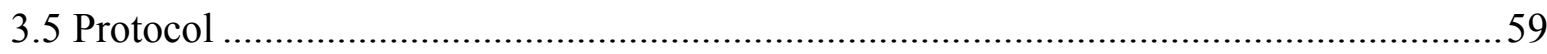

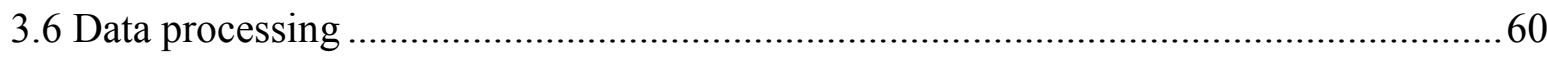

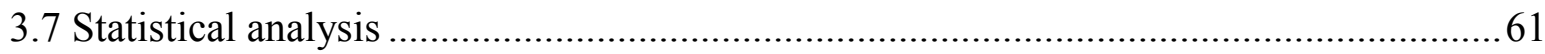

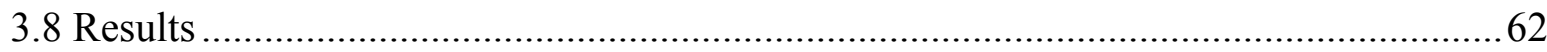

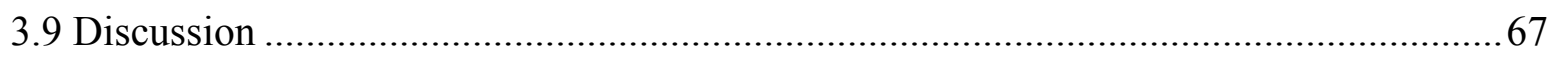

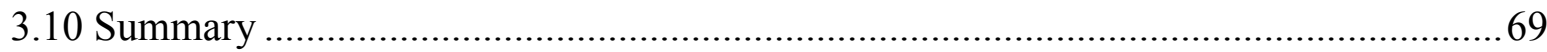

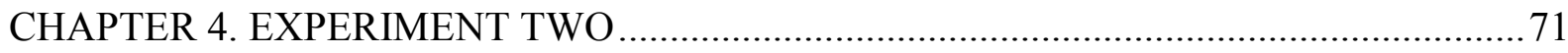

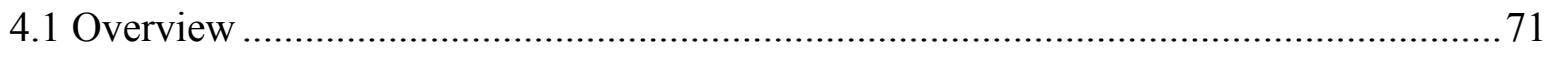

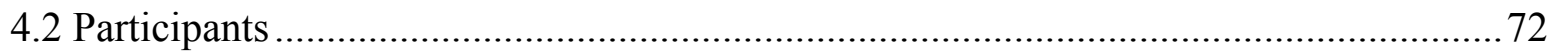

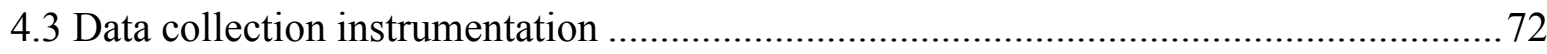

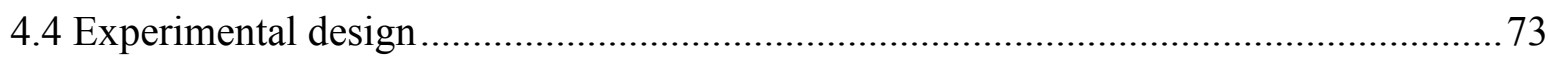

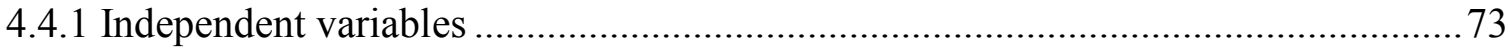

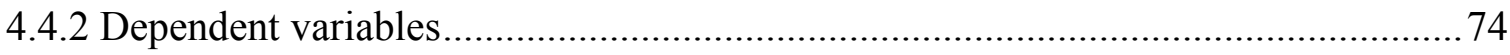

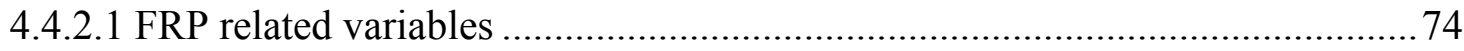

4.4.2.2 Lumbar segmental stability related variables................................................. 74

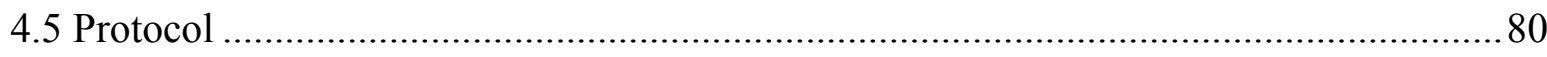

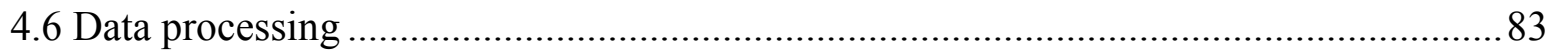

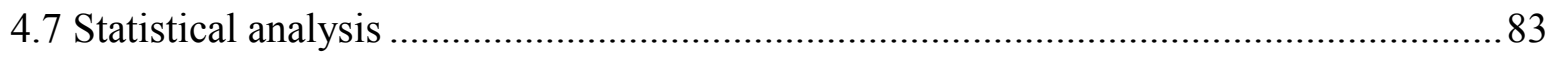




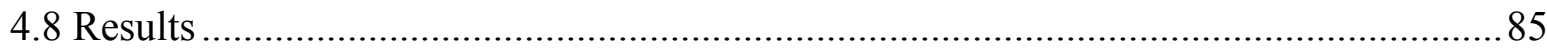

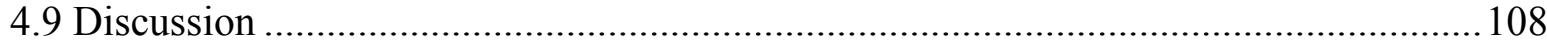

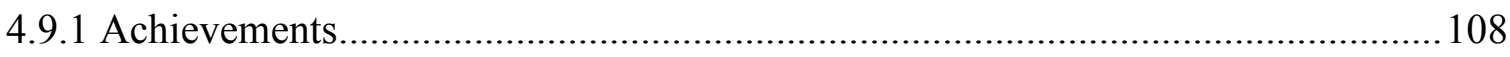

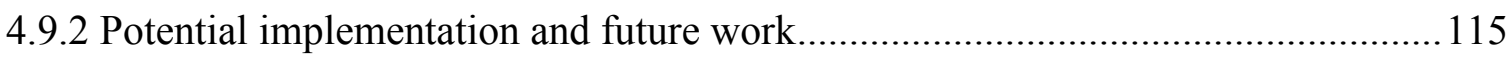

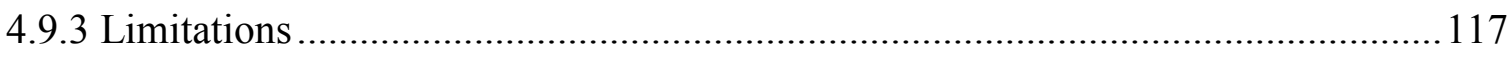

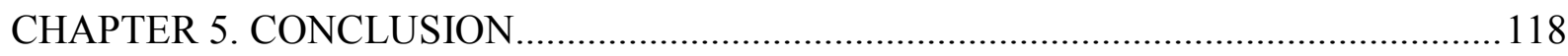

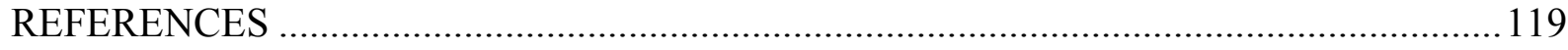

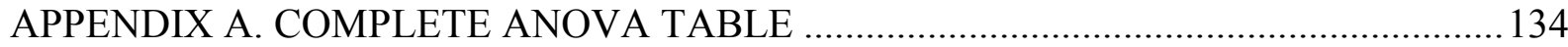

APPENDIX B. STATISTICAL MODEL ADEQUECY CHECKING .............................. 145

APPENDIX C. EFFECTS OF GROUND ON SEGMENTAL STABILITY (SUPPLEMENT)

171

APPENDIX D. INFORMED CONSENT DOCUMENT ................................................ 184 


\section{LIST OF FIGURES}

Figure 1: Severity distribution of low back pain (Casside et al., 1998).................................5

Figure 2: MSDs incidence rates of typical construction tasks (Wang et al. 2015) .................. 14

Figure 3: An illustration of human spine (from 2015 Gray's Anatomy)................................. 19

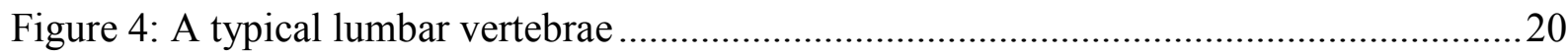

Figure 5: Intervertebral disc illustration (from 2015 Gray's Anatomy) ...............................20

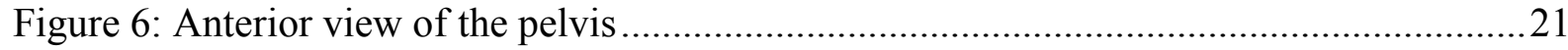

Figure 7: An illustration of transversus abdominis (TrA), obliquus internus abdominis (OI)

and obliquus externus abdominis (OE) (photo credit: www.brentbrookbush.com) ................25

Figure 8: A demonstration of spinal ligaments (from 2015 Gray's Anatomy) .......................26

Figure 9: Fascial layers of the posterior abdominal wall (from 2015 Gray's Anatomy) .........27

Figure 10: A demonstration of concentric and eccentric muscle contraction for lumbar

extensor muscles

30

Figure 11: A demonstration of the "EMG-off" and "EMG-on" point of a back muscle and the

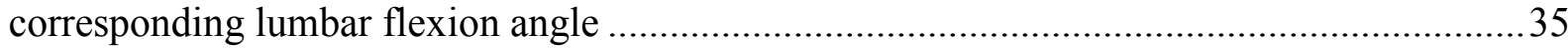

Figure 12: Demonstration of the six different stance conditions (Hu et al., 2014).................43

Figure 13: Examples to demonstrate the concept of stability (Reeves et al.,2007) ................45

Figure 14: Examples to demonstrate the concept of robustness (Reeves et al.,2007) .............46

Figure 15: A demonstration of sacrotuberous ligaments and sacroiliac ligaments (from 2015

Gray's Anatomy)

Figure 16: Side and frontal views of the slanted ground conditions: right panel, $30^{\circ}$ slanted;

middle panel, $15^{\circ}$ slanted; left panel, flat ground .............................................................53

Figure 17: (a) Delsys biopolar EMG electrodes; (b) EMG signal amplifier ..........................54

Figure 18: An illustration of the location of EMG sensors.................................................54

Figure 19: A demonstration of magnetic field based motion tracking system: (a) the transmitter which is the origin of the coordinate; (b) A/D board; (c) motion sensors; (d) an

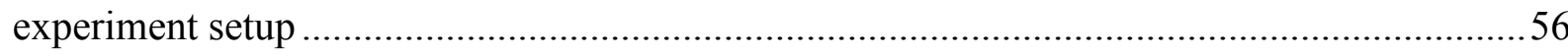

Figure 20: The dynamometer used in the current study ................................................57

Figure 21: Illustration of the definition of lumbar flexion angle and trunk inclination angle..59 
Figure 22: Maximum lumbar flexion angle under different ground conditions. Different letters denote angles that are statistically different from one another ........

Figure 23: Maximum trunk inclination angle under different ground conditions. Different

letters denote angles that are statistically different from one another

Figure 24: Lumbar EMG-Off angle for the ipsilateral side of erector spinae under different ground conditions. Different letters denote angles that are statistically different from one another.

Figure 25: Lumbar EMG-Off angle for the ipsilateral side of multifidus under different ground conditions. Different letters denote angles that are statistically different from one another ....65 Figure 26: Trunk EMG-Off angle for the ipsilateral side of erector spinae under different ground conditions. Different letters denote angles that are statistically different from one another. 65

Figure 27: Trunk EMG-Off angle for the ipsilateral side of multifidus under different ground conditions. Different letters denote angles that are statistically different from one another ....66 Figure 28: Mediolateral displacement of C7 sensor during trunk flexion motion. Positive value indicates rightward shift. Different letters denote displacements that are statistically different from one another .68

Figure 29: (a) side view of the $15^{\circ}$ downhill facing condition; (b) side view of the flat ground condition; (c) side view of the $-15^{\circ}$ uphill facing condition 73

Figure 30: An illustration of the sway motion profile of the motion sensor in one experimental trial .75

Figure 31: The demonstration of an asymmetric trunk bending task .82

Figure 32: A demonstration of how the trigger was used to determine a fully flexed trunk posture .82

Figure 33: The effect of ASYM on maximum lumbar flexion angle (MLF) ........................87

Figure 34: The effect of ASYM on maximum trunk inclination angle (MTI) ...................... 88

Figure 35: The effect of ASYM on right erector spinae EMG-off lumbar angle (RL3L).......88

Figure 36: The effect of ASYM on right erector spinae EMG-off trunk angle (RL3T)..........89

Figure 37: The effect of ASYM on right multifidus EMG-off lumbar angle (RL4L)............89

Figure 38: The effect of ASYM on right multifidus EMG-off trunk angle (RL4T).............. 90

Figure 39: The effect of GROUD on right erector spinae EMG-off lumbar angle (RL3L) .....91 
Figure 40: The effect of GROUD on right multifidus EMG-off lumbar angle (RL4L) ..........91

Figure 41: The effect of GROUD on right erector spinae EMG-off trunk angle (RL4L) ........92

Figure 42: The effect of GROUD on right multifidus EMG-off trunk angle (RL4L) ............ 92

Figure 43: Effects of GROUND on maximum lumbar flexion angle.................................. 93

Figure 44: Effects of GROUND on MTI angle under different ASYM conditions .................93

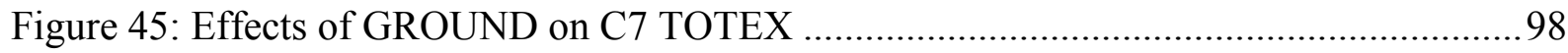

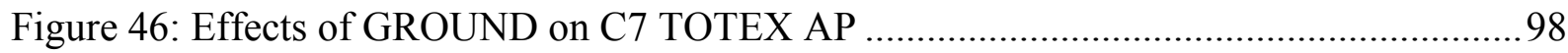

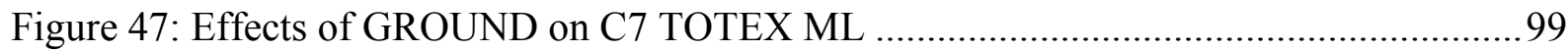

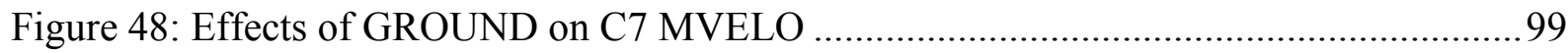

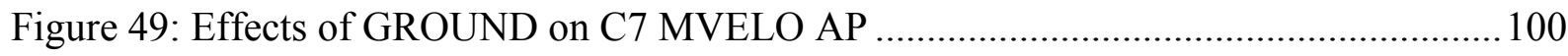

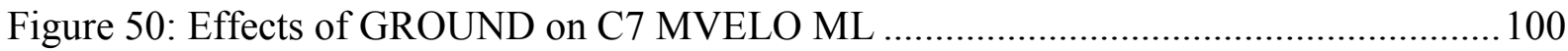

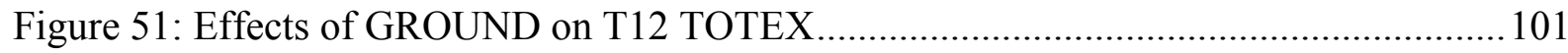

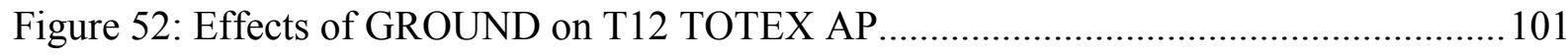

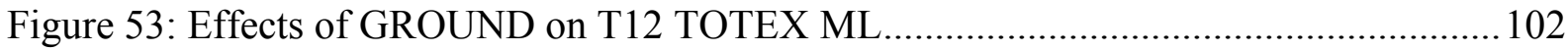

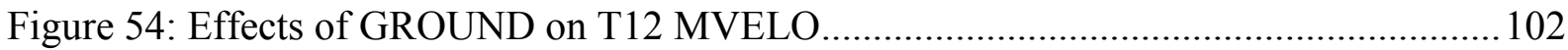

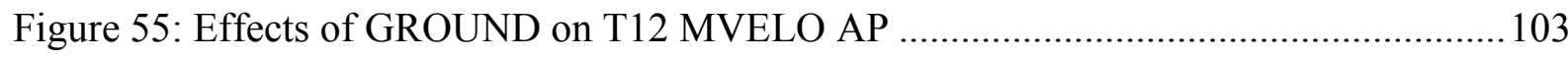

Figure 56: Effects of GROUND on T12 MVELO ML................................................. 103

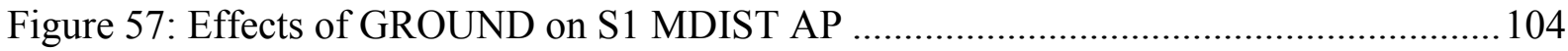

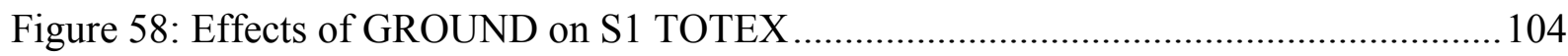

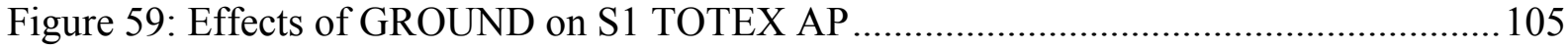

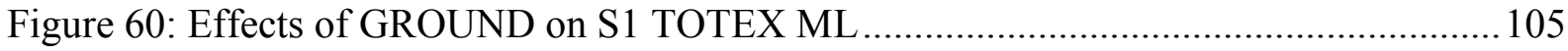

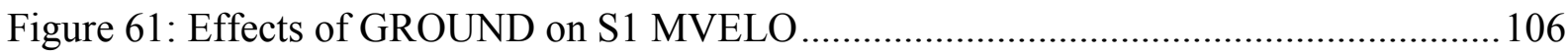

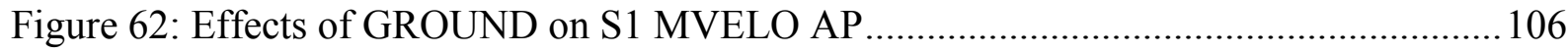

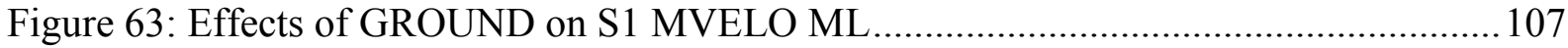

Figure 64: Effects of GROUND on S1 AREA SW .................................................... 107

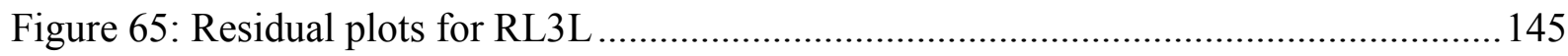

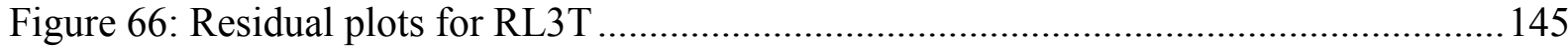

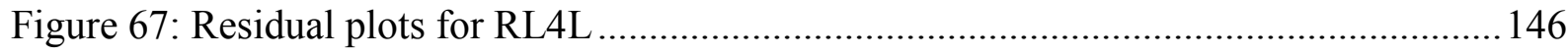

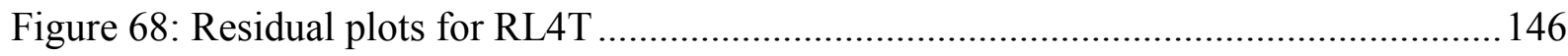

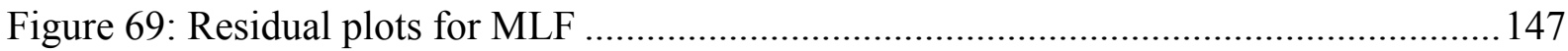

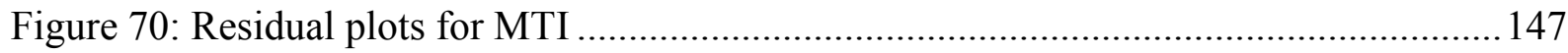




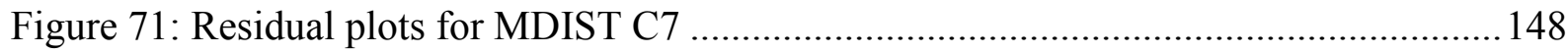

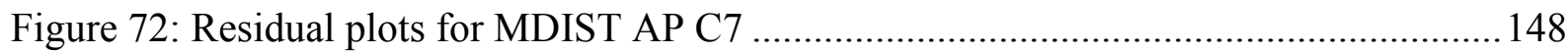

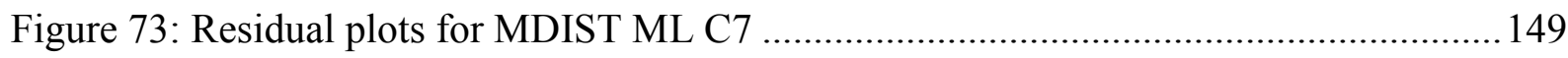

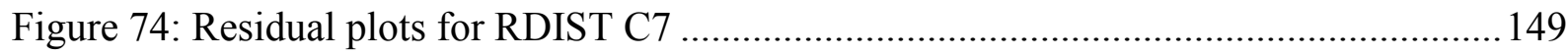

Figure 75: Residual plots for RDIST AP C7 ………........................................................ 150

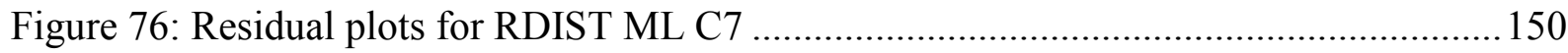

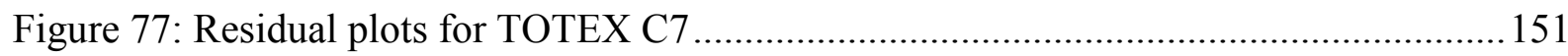

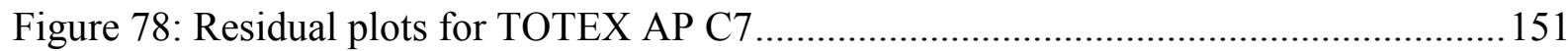

Figure 79: Residual plots for TOTEX ML C7 ………........................................................ 152

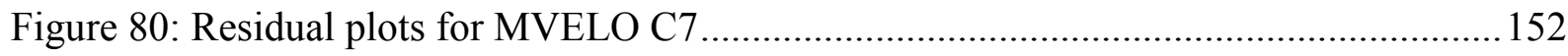

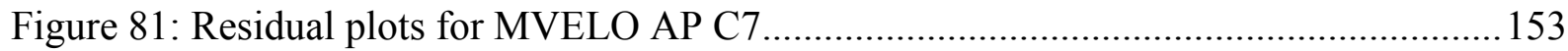

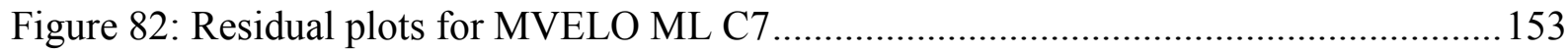

Figure 83: Residual plots for AREA CC C7 ....................................................................... 154

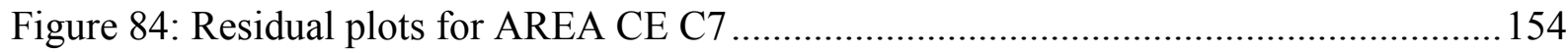

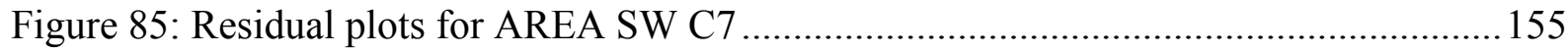

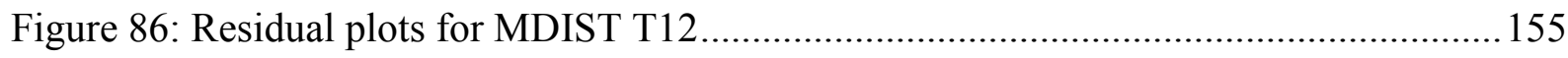

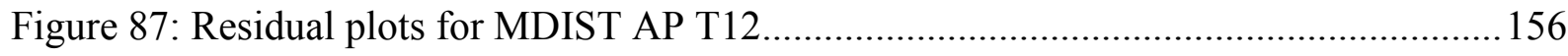

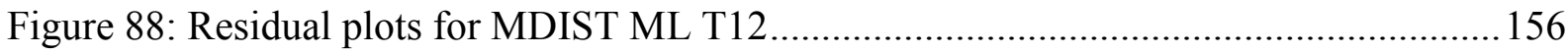

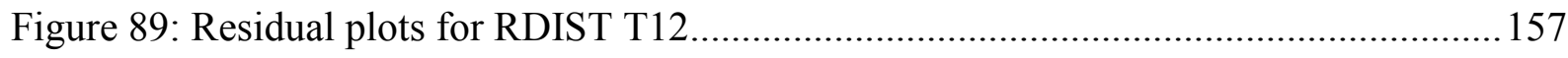

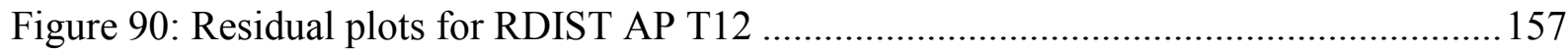

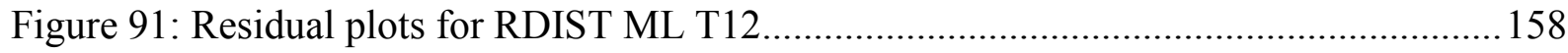

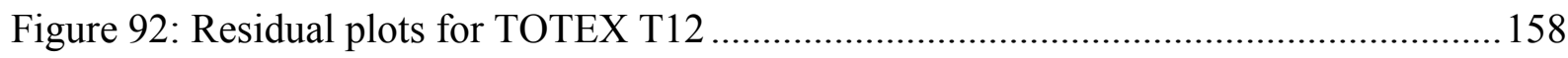

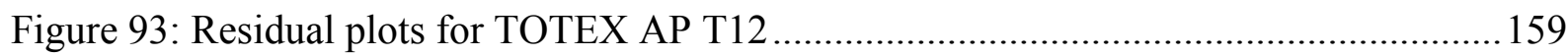

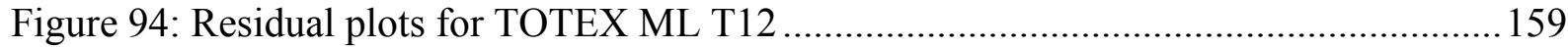

Figure 95: Residual plots for MVELO T12 …………...................................................... 160

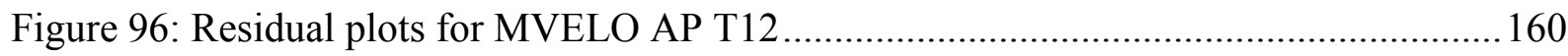

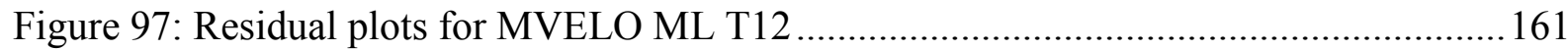

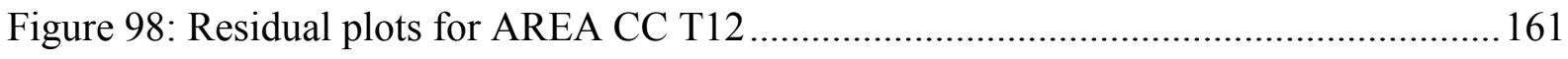

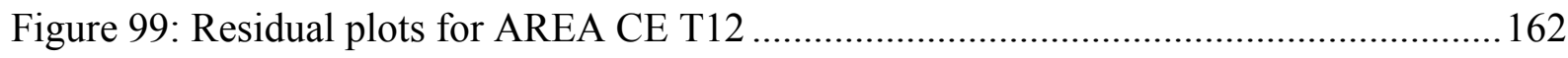

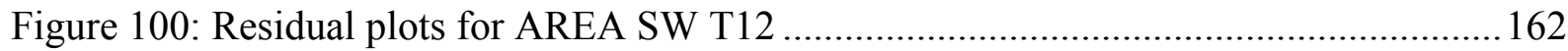

Figure 101: Residual plots for MDIST S1 …………....................................................... 163 


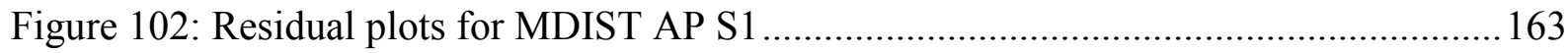

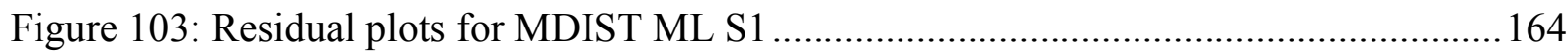

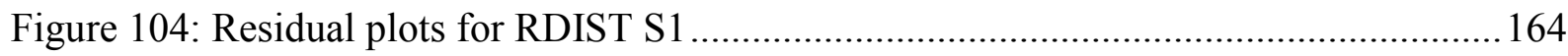

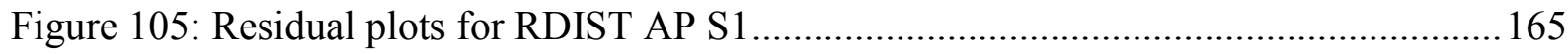

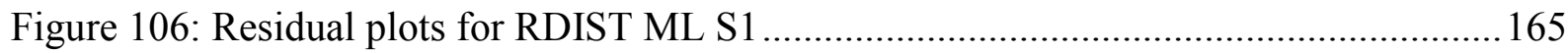

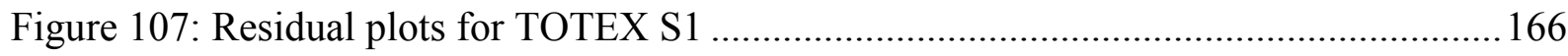

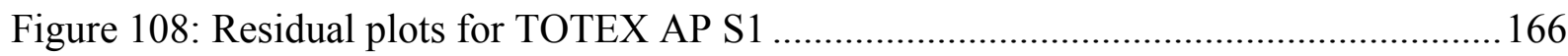

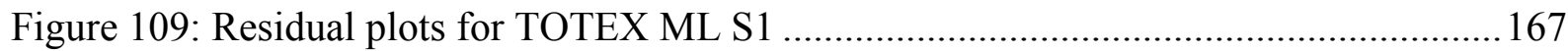

Figure 110: Residual plots for MVELO S1 ………........................................................... 167

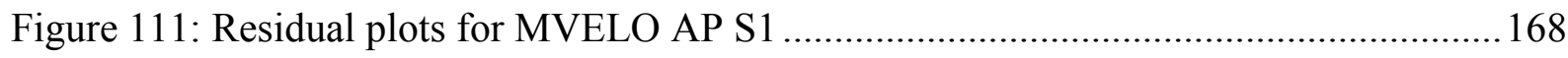

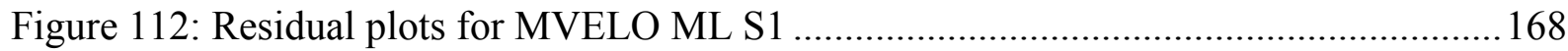

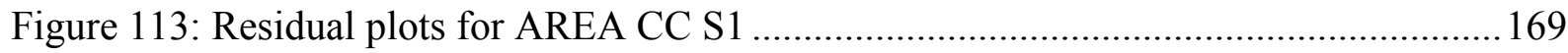

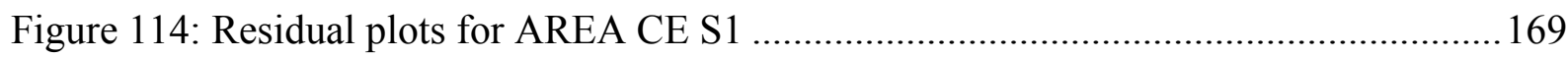

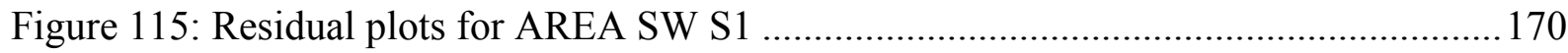

Figure 116: Effects of GROUND on C7 MDIST ………..................................................171

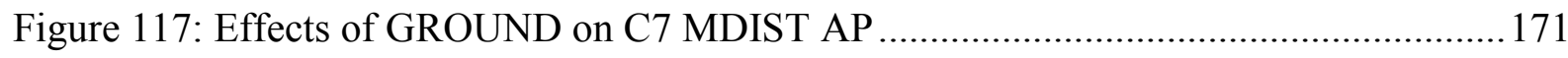

Figure 118: Effects of GROUND on C7 MDIST ML ........................................................172

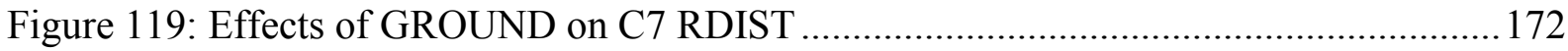

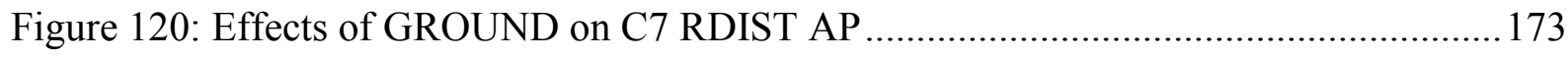

Figure 121: Effects of GROUND on C7 RDIST ML .......................................................... 173

Figure 122: Effects of GROUND on C7 AREA CC …………...........................................174

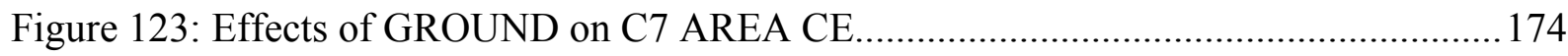

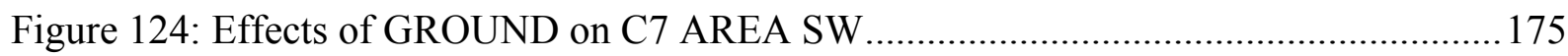

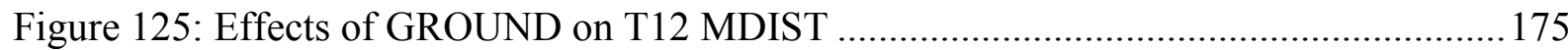

Figure 126: Effects of GROUND on T12 MDIST AP .......................................................176

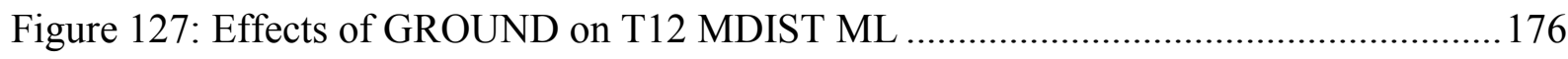

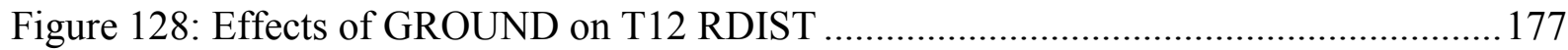

Figure 129: Effects of GROUND on T12 RDIST AP ........................................................ 177

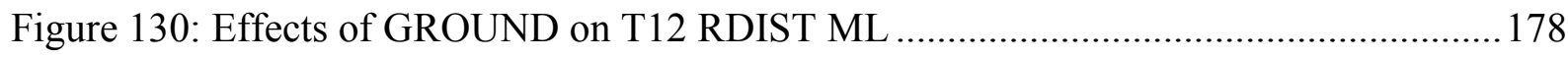

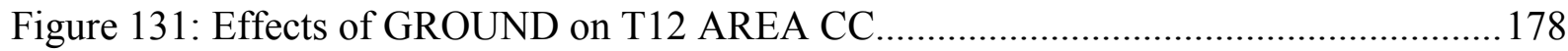

Figure 132: Effects of GROUND on T12 AREA CE.........................................................179 


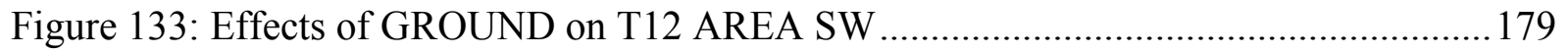

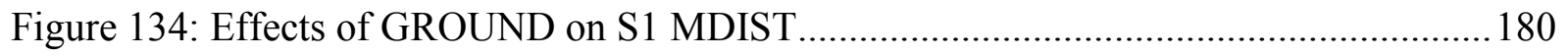

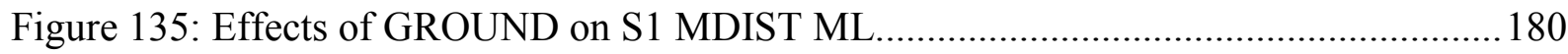

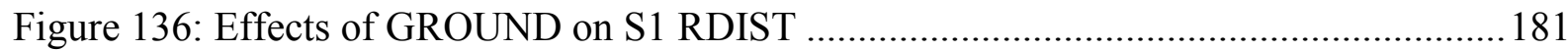

Figure 137: Effects of GROUND on S1 RDIST AP ................................................... 181

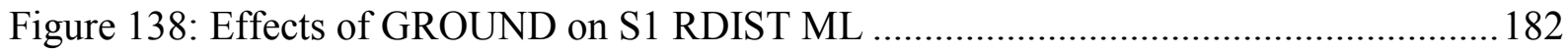

Figure 139: Effects of GROUND on S1 AREA CC ........................................................ 182

Figure 140: Effects of GROUND on S1 AREA CE .................................................... 183 


\section{LIST OF TABLES}

Table 1: Prevalence of LBP according to prevalence period and case definition variations

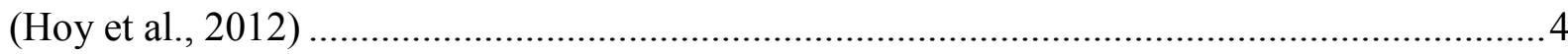

Table 2: Relative risk of LBP for occupational categories (Leigh \& Sheetz, 1989; Punnett et al., 2005)

Table 3: PCSA $\left(\mathrm{cm}^{2}\right)$ for each muscle. Bolded cells indicate a significant difference between different side (p-value < 0.05) (from Marras et al., 2001) .................................................23

Table 4: Methods for defining onset and cessation of FRP (Jin et al., 2012) .........................43

Table 5: The EMG-Off angle difference (right minus left) between erector spinae and

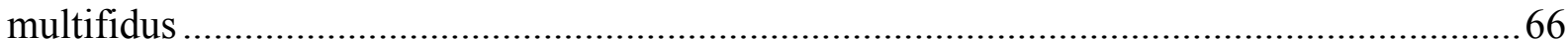

Table 6: Dependent variables in the lumbar stability category .......................................... 81

Table 7: Percentage of trials that showed FRP among the sampled lumbar muscles.............. 86

Table 8: The results of univariate ANOVA on FRP related variables ..................................86

Table 9: The results of univariate ANOVA on C7 segmental stability variables................... 94

Table 10: The results of univariate ANOVA on T12 segmental stability variables ................94

Table 11: The results of univariate ANOVA on S1 segmental stability variables .................95

Table 12: Effect of ASYM on C7 segmental stability .................................................... 96

Table 13: Effect of ASYM on T12 segmental stability …............................................. 96

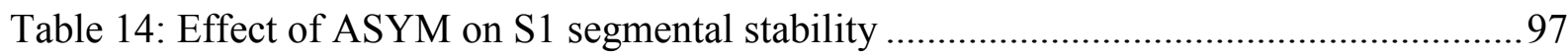




\section{CHAPTER 1. INTRODUCTION}

Low back pain (LBP) remains one of the most prevalent occupational health problems worldwide (Deyo et al., 2006). It is estimated that around $80 \%$ of the United States population experience at least one episode of LBP in their lifetime (Hellman \& Imboden, 2009) and the average age-related prevalence of persistent LBP is found around $15 \%$ in adults and the prevalence of benign back pain decreases with age after 60 (Dionne et al., 2006). LBP generates significant economic costs: LBP has been reported to response for more than 90 billion dollars of annual medical expenses (Luo et al., 2003). The expenditures for inpatient care was $\$ 27.9$ billion and it accounts for the largest proportion of the total expenditures (31\%), followed by the expenditures for the office-based visit (\$23.6 billion, 26\%).

LBP is a multifactorial problem with many possible causes and the exact etiology of LBP is still unclear. However, previous studies have identified a number of risk factors that are associated with LBP including occupational, non-occupational and psychosocial factors (Sitthipornvorakul et al., 2011; Manchikanti et al., 2009; Leigh \& Miller, 1997; Heuch et al., 2010). Among all the potential risk factors, uneven working surface is commonly seen in a number of different occupational settings such as agriculture, fishing and transportation. It has been reported that uneven surface could elevate the risk of getting LBP but the underling mechanism remains unknown.

The structure of the lumbar spine is very complex and mainly consists of two types of tissues: active tissues (e.g. contractile component of muscles) and passive tissues (non-contractile component of muscles, bone, ligaments and fascia discs). During sagittal bending motion a 
transition of load from active components to passive components occurs at deep trunk flexion postures (Ning et al., 2012). This synergy between lumbar active and passive tissues was termed as flexion relaxation phenomenon (FRP) by Floyd and Silver (Floyd \& Silver, 1955). Previous studies have made extensive efforts in investigating lumbar FRP while standing on flat and even ground surfaces (Sarti et al., 2001; Descarreaux et al., 2008; Shin et al., 2009; Alschuler et al., 2009) while the effect of slanted ground surface on the lumbar FRP has not been well understood. Furthermore, spine stability is critical for the human central neural system to control body motion and avoid injury (Gurfinkel et al., 1995; Hlavacka et al., 1996; Reeves et al., 2007). It is of importance to know how uneven ground surface can influence the spinal stability and knowledge regarding the spinal stability under uneven surface could be used to prevent low back injuries.

Therefore, the purpose of the current research was to investigate the influence of uneven ground surface on the spinal biomechanical responses. To be specific, we examined how lumbar muscle FRP was affected when performing trunk bending motions on slanted ground surfaces. At the same time, spinal stability was also evaluated and compared between different conditions. It was hypothesized that when standing on a slanted surface, alternations to lumbar active and passive tissue sharing mechanism could significantly affect the FRP performance and the spinal segmental stability. 


\section{CHAPTER 2. BACKGROUND}

\subsection{Low back disorders}

\subsubsection{Prevalence}

Low back pain (LBP) remains the leading health problem amongst the population and a principal cause of disability that influences work performance (Muslim et al., 2013). According to a recent global review (Hoy et al., 2012), the one year LBP prevalence rate was approaching 38.0\% while the lifetime prevalence rate was $39.9 \%$. LBP was shown to be a major health problem worldwide with the highest incidence among female and those ages between 40 to 80 years. The same study also indicated that the total number of people in the population with LBP is likely to keep increasing considerably over the coming years. Overall, the annual prevalence of chronic low back pain has been reported to range from $15 \%$ to $45 \%$, with a point prevalence of $30 \%$ (Manchikanti et al., 2009) (Table 1).

It has been reported that the average age-related prevalence of persistent LBP is around $15 \%$ in adults and $27 \%$ in the elderly and the prevalence of benign back pain decreases with age after 60 while the prevalence of severe back pain continues to grow with age (Dionne et al., 2006). The occurrence of LBP is also distributed among different types of occupations. One study used a nationwide survey and obtained 1417 participants' responses of their job and physical wellness (Leigh \& Sheetz, 1989). The job group with the lowest risk level was set as the reference and all other groups were compared with it. By this means, relative risk index for all jobs were generated. The same method was adopted by another study (Punnett et al., 2005) and the results were summarized in Table 2. 
Table 1: Prevalence of LBP according to prevalence period and case definition variations (Hoy et al., 2012)

\begin{tabular}{|c|c|c|c|c|c|c|c|c|}
\hline \multirow[b]{2}{*}{ Prevalence } & \multirow[b]{2}{*}{$\begin{array}{l}\text { No. of } \\
\text { estimates }\end{array}$} & \multicolumn{5}{|c|}{ Quantile } & \multirow[b]{2}{*}{$\begin{array}{l}\text { Mea } \\
\text { n }\end{array}$} & \multirow[b]{2}{*}{$\begin{array}{l}\text { SD } \\
\%\end{array}$} \\
\hline & & $\begin{array}{l}10 \\
\%\end{array}$ & $\begin{array}{l}25 \\
\% \\
\end{array}$ & $\begin{array}{l}50 \\
\% \\
\end{array}$ & $\begin{array}{l}75 \\
\%\end{array}$ & $\begin{array}{l}90 \\
\%\end{array}$ & & \\
\hline \multicolumn{9}{|l|}{ Prevalence Period } \\
\hline Point & 243 & 6.3 & 10.3 & 15 & 24.2 & 35.5 & 18.3 & 11.7 \\
\hline 1 month & 145 & 14.8 & 21.3 & 32.1 & 38 & 49 & 30.8 & 12.7 \\
\hline 1 year & 271 & 14.3 & 21 & 37.4 & 53 & 64.8 & 38 & 19.4 \\
\hline Lifetime & 133 & 6.2 & 15.1 & 42 & 60.4 & 66.4 & 38.9 & 24.3 \\
\hline \multicolumn{9}{|l|}{ Anatomic } \\
\hline Back & 268 & 9.9 & 15.8 & 26.6 & 36.4 & 53.6 & 28.5 & 16.4 \\
\hline Low back & 302 & 7.2 & 12.8 & 26.1 & 43.1 & 56 & 29.1 & 18.8 \\
\hline R12 to lower GFs' & 254 & 11 & 17.4 & 35.2 & 52 & 63.7 & 35.5 & 19.7 \\
\hline \multicolumn{9}{|l|}{$\begin{array}{l}\text { Minimum episode } \\
\text { duration }\end{array}$} \\
\hline Not specified & 661 & 8.7 & 15 & 31.5 & 48.8 & 62.5 & 33.2 & 20.3 \\
\hline 1 day & 146 & 14.1 & 22.1 & 34 & 44 & 56.4 & 33.8 & 15.8 \\
\hline 3 months & 86 & 8.7 & 12.8 & 19.2 & 24.3 & 33.6 & 20.1 & 9.8 \\
\hline \multicolumn{9}{|l|}{ Activity limitation } \\
\hline With or without activity & 912 & 9.1 & 15.8 & 29.1 & 45.5 & 58.2 & 31.8 & 19 \\
\hline Activity limiting only & 54 & 5 & 8.1 & 12.2 & 18.8 & 30.8 & 17 & 15.4 \\
\hline
\end{tabular}

Table 2: Relative risk of LBP for occupational categories (Leigh \& Sheetz, 1989; Punnett et al., 2005)

\begin{tabular}{l|c|c|c}
\hline Occupational category & $\begin{array}{c}\text { Relative risk (95\% CI) } \\
\text { (Leigh \& Sheetz 1989) }\end{array}$ & $\begin{array}{c}\text { Relative risk (95\% CI) } \\
\text { (Punnett et al., 2005) }\end{array}$ & $\begin{array}{c}\text { Exposure category } \\
\text { used }\end{array}$ \\
\hline $\begin{array}{l}\text { Managers and } \\
\text { professionals }\end{array}$ & $1.00(\mathrm{NA})$ & 1.00 & Background \\
\hline Clerical or sales worker & $1.38(0.85-2.25)$ & 1.38 & Low \\
\hline Operators & $2.39(1.09-5.25)$ & 2.53 & Moderate \\
\hline Service workers & $2.67(1.26-5.69)$ & 2.53 & Moderate \\
\hline Farmers & $5.17(1.57-17.0)$ & 3.65 & High \\
\hline
\end{tabular}


In an epidemiologic comparison of LBP patients, Cassidy et al. divided all patients into four grades, from Grade I to Grade IV based on their disease severity and disability (Cassidy et al., 1998): Grade I described low-intensity/ low-disability LBP, Grade II described highintensity/low-disability LBP, and Grades III and IV described high-intensity/high-disability LBP. Results of this study showed that $48.9 \%$ patients experienced Grade I, $12.3 \%$ experienced Grade II, and $11 \%$ of the patients had Grade III and Grade IV pain levels (Figure 1).

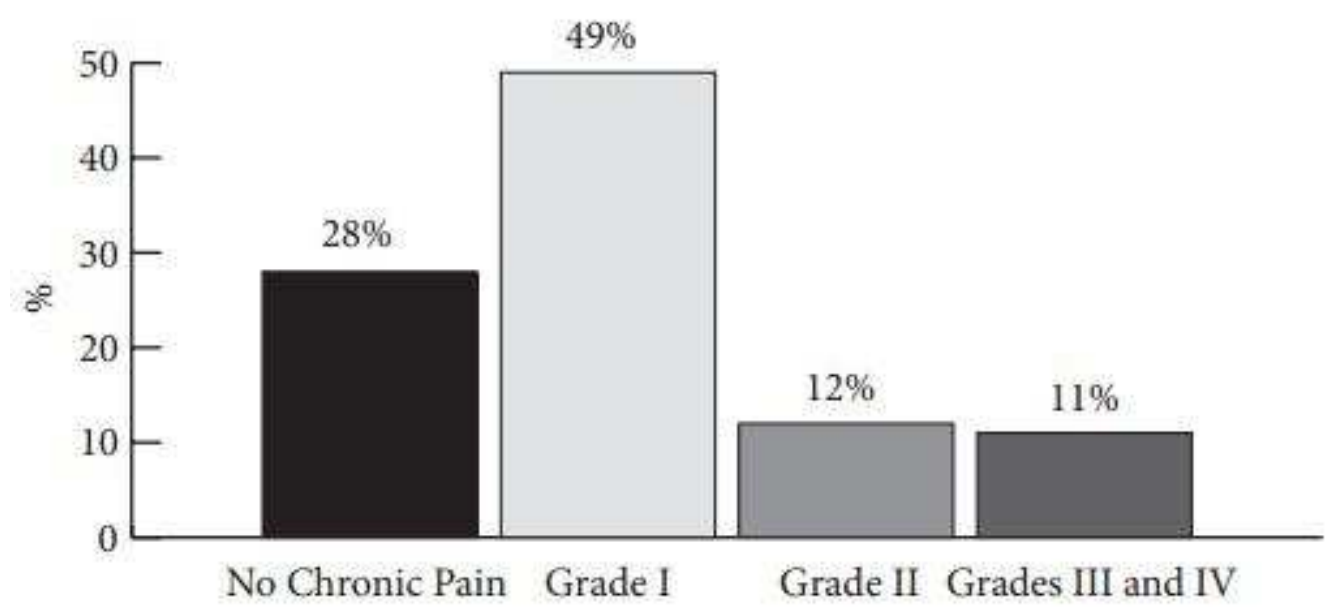

Figure 1: Severity distribution of low back pain (Casside et al., 1998)

In terms of the prevalence of occupational LBP, one study indicated that the percentage of the work force being affected varied from $2 \%$ to $8 \%$ with days of absence from work per person per year ranges from 9 days in the United States, to 10 days in West Germany, 20 days in Canada and Great Britain, 25 days in the Netherlands and 40 days in Sweden (Nachemson, 1992). In addition, It has been estimated that almost $28 \%$ of the United States industrial population will experience disabling LBP at some time in their lifetime and $8 \%$ of the entire working population will be disabled in any given year, contributing to two fifth of all lost work days (Manchikanti et al., 2009; Leigh, 2011). 
A study reported that bask pain is an extremely common presenting complaint that occurs in upward of $80 \%$ of persons and the natural history of acute episodes of back pain in favorable in most patients. Evaluation of these patients includes performing a comprehensive physical examination and in many cases, obtaining imaging in the form of plain radiographs and MRI (Patrick et al., 2016; Shmagel et al., 2016).

In contradiction to the popular belief that the prevalence of LBP is steady or even declining, many studies have reported an increasing prevalence of LBP. For instance, one study showed the rising prevalence of LBP following an evaluation in 1992 and repeated in 2006 (Freburger et al., 2009). Results from that study indicated an increase in the prevalence of chronic impairing LBP over a 14 year interval, from $3.9 \%$ in 1992 to $10.2 \%$ in 2006 . Increases were observed for all adults, regardless of their gender, race, the symptom severity or the general health condition. Moreover, the proportion of individuals who sought care from a health care provider in the past year of the studies increased from $73.1 \%$ to $84.0 \%$, whereas the mean number of visits to all health care providers was roughly similar.

Similar to the increase in the prevalence of LBP, national statistical data showed that the proportion of population receiving Social Security Disability Income claiming musculoskeletal disease as the reason of disability also increased considerably from $8.2 \%$ in 1960 and $15.2 \%$ in 1992 to $33.8 \%$ in 2011 (Social Security Administration, 2011). It has been suspected that the increase in the use of health care services for chronic LBP is due to the pursuit of increased health care in the form of surgical and other interventions by patients with the condition (Deyo et al., 2006). 


\subsubsection{Health and economic impact}

LBP is associated with significant economic costs. One study indicated that LBP is responsible for more than 90 billion dollars of annual medical expenses (Luo et al., 2003). Specifically, the expenditures for inpatient care was $\$ 27.9$ billion and it accounts for the largest proportion of the total expenditures $(31 \%)$, followed by the expenditures for the office-based visit ( $\$ 23.6$ billion, 26\%). In addition, prescription drugs ( $\$ 14.1$ billion) and outpatient service ( $\$ 11.9$ billion) account for $15.6 \%$ and $13.1 \%$ accordingly. A more recent estimates of the economic burden of LBP in the United States, encompassing both direct and indirect costs was more than $\$ 84.1$ billion annually (Gore et al., 2012).

One study investigated the economic cost of pain in the United States using the 2008 Medical Expenditure Panel Survey (Gaskin \& Richard, 2012). Results showed that the estimated total cost was around $\$ 600$ billion in year 2010 . Additional health care cost due to pain ranged around $\$ 280$ billion. Moreover, the estimated value of lost productivity due to pain was roughly $\$ 315$ billion. The annual cost of pain was even greater than diabetes ( $\$ 188$ billion), cancer $(\$ 243$ billion) and heart disease ( $\$ 309$ billion). In addition, this estimation is conservative because it did not include the direct cost associated with pain for nursing home residents and persons who were incarcerated.

A study that has evaluated the economic cost of occupational injury in the United States showed the costs were more than $\$ 290$ billion (Leigh, 2011). This study also concluded that the direct and indirect costs of occupational injuries were at least as large as the cost of cancer and the 
percentage of occupation related injuries to the total cost of medical care are higher than generally assumed.

Another study examined the health care expenditures in the United States of treating spine (back and neck) disorders and the analyses results showed the total cost for United States adults with spine problems increased 65\% from 1997 to 2005 (Martin et al., 2008). In a follow up study from the same research group, authors concluded that from 1997 to 2006 the adjusted per-person expenditure was the largest component in terms of increasing total cost (Martin et al., 2009).

Moreover, it has been reported that all occupational costs are not covered by the current workers' compensation system: one study evaluated occupational disorder and workers' compensation date and results showed that around 46000 to 93000 deaths and $\$ 8$ billion to $\$ 23$ billion of medical costs were not attributed to the current workers' compensation system. This portion of occupational cost represented substantial portion that shifting from the workers' compensation system to individual workers, their families, private medical insurance and taxpayers (Leigh \& Robbins, 2004). Finally, disability secondary to chronic LBP is also considerable (Ricci et al., 2006; Walker et al., 2003).

\subsubsection{LBP risk factors}

LBP is a multifactorial problem with many possible etiologies. Many epidemiologic researches have attempted to evaluate LBP from different aspects, including biomechanical, occupational, non-occupational and psychosocial, etc. 


\subsubsection{Biomechanical factors}

It has been reported that peak lumbar compression and shear forces are closely associated with LBP (Kerr et al., 2001). To be specific, shear forces in the anterio-posterial direction act transversely across the lumbar spine to resist the tendency of the top parts of the torso to slide forward relative to the lower parts. Elevated spinal shear force can result from a forward-inclined torso, particularly with extra weights in the hands or from pulling actions in an upright posture. Compression and shear forces could also initiate new inflammation or aggravate existing problems in the facet joint region including the annular fibers of the discs (Norman et al., 1998).

Marras and colleagues assessed the contribution of three-dimensional dynamic trunk motions to the risk of LBP during occupational lifting in industry (Marras et al., 1993). To that end, more than 400 repetitive industrial lifting jobs were examined and a triaxial electrogoniometer was used to measure the angular position, velocity and acceleration characteristics of the lumbar spine during the performance of each task. Results indicated that there were five factors could contribute to the high risk of LBP: 1) lifting frequency; 2) load moment; 3) trunk lateral velocity; 4) trunk twisting velocity and 5) the trunk sagittal angle.

Low back muscle fatigue has long been recognized as a risk factor for LBP. It has been reported that low back muscle fatigue could weakens the muscular support to the spinal structure (Gardner-Morse et al., 1995) and reduces muscle stiffness (Golhoffer et al., 1987). These alternations could result in increased mechanical stress to the spinal functional component (De Beeck \& Hermans, 2000) and this alternation is highly associated with LBP. One previous study has concluded that the magnitude of spinal loading is significantly associated with the 
occurrence of LBP since high spinal loading may over time degenerate the intervertebral disc, resulting in annulus fibrosus bulges or ruptures that may press on spinal nerve roots and result in LBP (Neumann et al., 1999).

Viscoelastic changes of lumbar passive tissues under different conditions could also elevate the LBP risk level (Solomonow et al, 2003). One study reported that passive spine stiffness decreased significantly after prolonged flexion (Adams and Dolan, 1996). In other words, the mechanical properties of lumbar passive tissue can be influenced by prolonged stretching: changes in the collagen network and its relation with the surrounding proteoglycans in the lumbar ligaments were concluded to be contributable for the reduction of spine stiffness. Other researches indicated that the mechanical properties changes of lumbar passive tissue could increase the laxity of the spine, reduce the spine stability and result in unstable joint behavior (Panjabi 1992a; Panjabi 1992b). Similarly, Bazrgari and colleagues examined the effects of creep deformation on trunk behaviors and concluded that the spinal stability reduced after creep deformation such reduction might be due to the fact that the alterations in reflexive trunk behavior following creep deformation exposures was not adequate to compensate the reductions in intrinsic stiffness which is caused by the creep deformation (Bazrgari et al., 2011).

In addition, employment and workplace factors, both physical and psychological, have been reported to influence the prevalence of LBP. Pushing, pulling and prolonged walking were found to be predictors of future back pain (Sitthipornvorakul et al., 2011). Vehicle driving had been associated with a higher incidence of back symptoms and was attributed to the effects of wholebody vibration on the intervertebral disc (Manchikanti et al., 2009; Leigh \& Miller, 1997). 


\subsubsection{Psychological factors}

The influence of psychological distress in the development of low back pain has been studied by a number of researchers. Factors such as anxiety and depression, kinesophobia (i.e. the fear of movement) and somatization (i.e. the expression of distress as physical symptoms) have been suggested as risk factors of LBP (Manchikanti et al., 2012; Mannion et al., 1996). For example, one review study suggested that the perceived stress by workers may become a factor in the process of developing musculoskeletal disorders (Bongers et al., 1993). By modifying the lifting posture and lifting maneuver (i.e., lifting kinematics and kinetics), mechanical load on spine could be influenced.

\subsubsection{Individual factors}

A number of studies have reported that the body mass index may be linked to LBP. For instance, in one review study, Leboeuf-Yde investigated whether body weight is associated with LBP and results indicated that among all 65 studies reviewed, 32\% reported a statistically significant positive association between body weight and LBP (Leboeuf-Yde 2000). In a cross-sectional study of the effects of body mass index (BMI) on the prevalence of LBP, results demonstrated that in both genders, a larger BMI was significantly associated with an increased prevalence of LBP (Heuch et al., 2010).

In addition, smoking is an important life style factor that can affect prevalence of LBP. One study reported a positive association between smoking and LBP and that association increased with the duration of LBP (Leboeuf-Yde et al., 1998). Additionally, Leboeuf-Yde performed a systematic review of the literature on LBP and smoking (Leboeuf-Yde 1999). This study covered 
47 previous epidemiologic studies and analyses results showed consistent evidence that smoking and LBP have close connection.

It has been stated that LBP usually starts at the early stage of life with the highest frequency of symptoms occurring in the age range from 35 to 55 and the symptom duration increase with age (Cecchi et al., 2006; Dionne et al., 2006; Stanton et al., 2008). Increasing age has been associated with an increase in musculoskeletal disorders. A United States national survey revealed that back pain is the third commonly reported symptom in general and the most commonly reported in the musculoskeletal system (Koch \& Smith, 1985).

In addition, LBP has been reported consistently in a higher proportion of female than male (Hoy et al., 2012) and LBP appears to be a significant problem during pregnancy and often remains after delivery (Kristiansson \& Schoultz, 1996). Systematic reviews have indicated that the risk factors most commonly assessed include: previous LBP history before pregnancy, smoking habits, mother's height, weight, number of previous pregnancies and the use of epidural anesthesia or cesarean delivery during previous labor (Battie et al., 1995).

\subsection{Uneven ground condition as an occupational injury risk factor}

\subsubsection{Uneven ground in occupational environment}

Although flat and balanced ground surfaces are common in indoor occupational settings, uneven ground surfaces are commonly seen in many outdoor based industries. Steep slope and height are typical characteristics of roof environments and construction industry (Simeonov et al., 2003; Wang et al., 2015). Figure 2 lists most common construction tasks and a majority of those are 
related with uneven working surface and high MSDs incidence rate (Figure 2). In addition, agriculture industry (Zhao et al., 1987), fishing industry (Ning \& Mirka, 2010; Mirka et al., 2011) and transportation industry (Andres et al., 2005) also involve substantial amount of uneven working surface.

\subsubsection{Uneven ground and falling}

Falling in the workplace is a major reason for occupational injuries and fatalities. Falling remains the second most common cause of occupational fatalities and accounts for $14 \%$ of the fatal occupational injuries in the United States (BLS, 2007a). Falling is the third most common cause for non-fatal injuries that involving days away from work, accounts for nearly $20 \%$ of the 1183500 injuries in 2006 (BLS, 2007b). It has been reported that falling from roof accounts for one third of all fatal injuries in the construction section (BLS, 1999). Typical falling injuries are usually extremely severe which would require long recovery and treatment periods and result in considerable amount of medical cost (Gillen et al., 1997). Losing balance has been reported to be one of the major causes of falling injuries and uneven surfaces can significantly influence human balance performance. Therefore, substantial amount of studies have been conducted to explore the effects of uneven surface on human balance. 


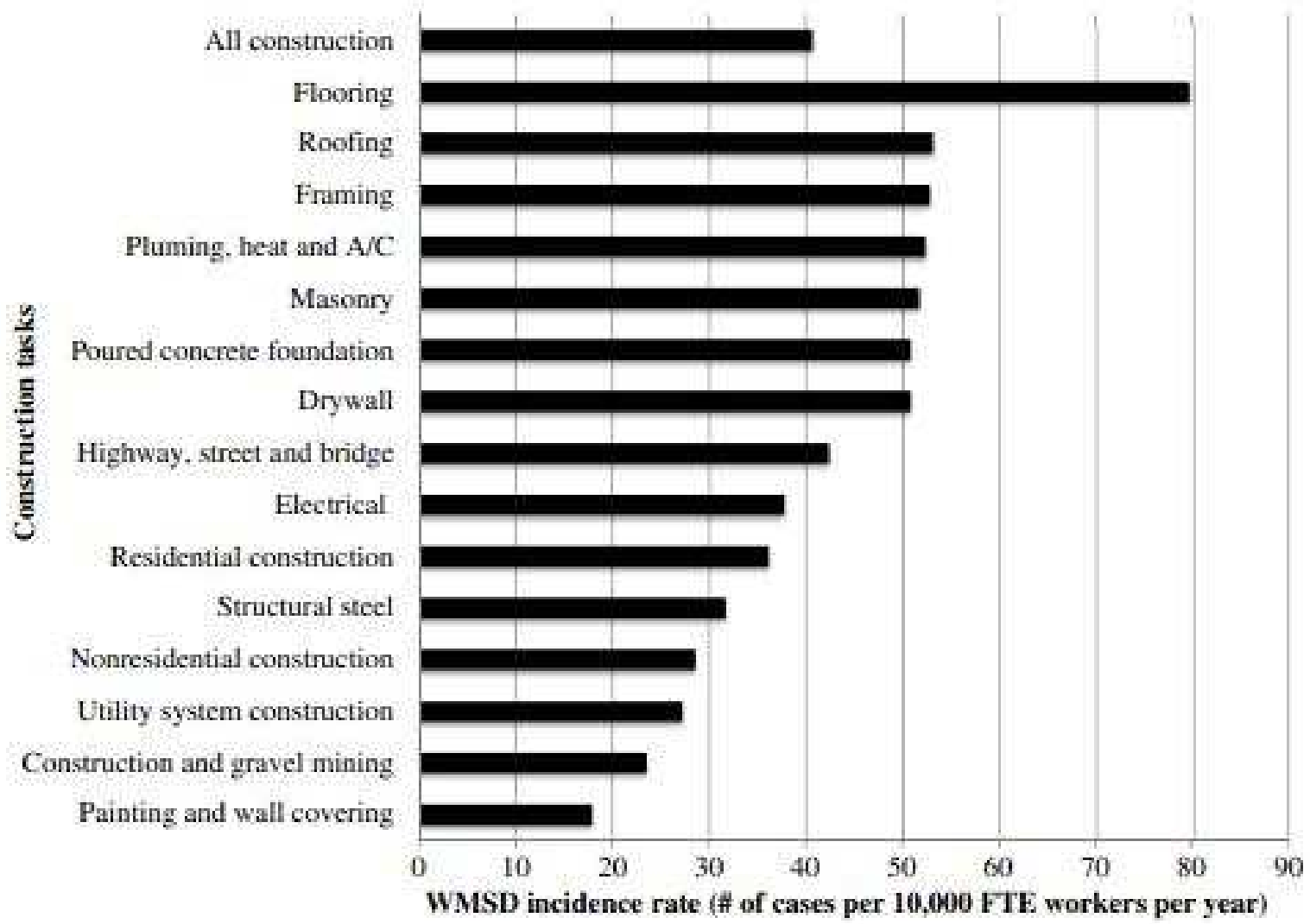

Figure 2: MSDs incidence rates of typical construction tasks (Wang et al. 2015)

One study evaluated the risk of slipping during material handling in agriculture industry (Zhao et al., 1987). Eight participants were recruited to conduct leg lifts and back lifts on two uneven surfaces $\left(15^{\circ}\right.$ and $\left.25^{\circ}\right)$ while their ground reaction force and path of the weighted box were recorded. Participants' subjective slipping potential indexes were also reported. Analyses results demonstrated that larger slope angle would increase the risk of slipping significantly and subjective slipping index also supported this finding.

Simeonov and colleagues investigated the effects of the roof slope magnitude on standing balance among construction workers (Simeonov et al., 2003). Twenty four participants were recruited to perform static standing tasks on 4 anterior-posterior slopes $\left(0^{\circ}, 18^{\circ}, 26^{\circ}\right.$ and $\left.34^{\circ}\right)$ in a 
laboratory environment while their postural sway characteristics were recorded with a force plate. Results showed that sloped surfaces in roof work environments significantly increased workers' standing postural instability. A more recent follow up study by the same research group concluded that the medial-lateral direction instability also increased with increase of slope when participants were standing on a semi-lateral slope-related surface (Simeonov et al., 2009).

One study evaluated joint kinematics (e.g. ankle, knee and hip) and muscle activation pattern alternation when standing on inclined surface (Mezzarane \& Kohn, 2007). Participants stood in quiet stance over a force platform positioned in one of three positions (horizontal, $14^{\circ}$ toes-up and $14^{\circ}$ toes-down) while their center of pressure and electromyogram of the lower extremity muscles were recorded and analyzed. Results indicated that standing over an inclined surface could induce changes in both short-term and long-term postural control. A more recent study conducted by Sasagawa and colleagues reported similar findings (Sasagawa \& Ushiyama, 2009).

Furthermore, one study examined the effects of surface inclination angle, standing direction and lumbar extensor muscle fatigue on postural control during quiet standing. In that particular study, three inclination angles $\left(0^{\circ}, 18^{\circ}\right.$ and $\left.26^{\circ}\right)$ and three standing directions (toes-up, toes-down and lateral facing) were tested and sixteen participants were involved (Lin \& Nussbaum, 2012). Center of pressure time series and subjectively perceived stability were obtained to calculate several postural control measurements. Results showed that adverse effects of standing on uneven surface differed in three different standing directions. In the lateral facing direction, a dose-response function could be obtained and tasks performed in a laterally facing direction could introduce higher falling risks. 


\subsubsection{Uneven ground and musculoskeletal disorders}

Uneven ground surface can also alter the behavior and lumbar musculature, biomechanical loading of the spine and influence musculoskeletal disorders (MSDs) risk level. One study investigated effects of the antero-posteriorly sloped ground on spinal loading (Shin \& Mirka, 2004). Participants' whole body motions were recorded as they lifted a $10 \mathrm{~kg}$ weight on 5 different surfaces $\left(10^{\circ}\right.$ and $20^{\circ}$ facing uphill, $10^{\circ}$ and $20^{\circ}$ facing downhill and flat ground). Then a two dimensional (2D) multi-segments top down model was used to evaluate the influence of slope surfaces on the L5/S1 joint moment. Results of that study showed in the freestyle lifting condition the maximum L5/S1 joint moment was considerably larger for the inclined surface compared to flat ground and declined surface. Collectively, this study concluded that the ground slope condition could influence the lifting kinematics and kinetics and needs to be considered in occupational environment.

Jiang et al. quantified the biomechanical responses while lifting on a laterally slanted ground (Jiang et al., 2005). Participants were asked to perform both isometric and dynamic weight lifting ( $40 \%$ of their maximum capacity) while stepping on four different conditioned platforms $\left(0^{\circ}, 10^{\circ}\right.$, $20^{\circ}$ and $30^{\circ}$ laterally tilted). Their whole body kinematics data were exported to a biomechanical model in order to estimate the L5/S1 joint moment and lateral force. Results of the isometric weight holding task showed that when participants stood on uneven surface, their left lumbar extensor muscle had 26\% higher electromyography activity and right lumber extensor muscle showed $70 \%$ higher electromyography level. In addition, results of the dynamic lifting tasks showed an increase in the instability producing lateral forces with the increase of slant magnitude. 
Another study which investigated the effects of ground rolling motion on bilateral low back muscle activity during weight lifting on a simulated boat demonstrated clear differences between the bilateral trunk extensor muscles with the lateral angular displacement of the ground surface (Ning \& Mirka, 2010). In the same study, an elevated trunk muscle co-contraction was also observed which has been shown to increase spinal loading (Granata \& Marras, 1995) thereby elevate the risk of LBP (Neumann et al., 1999).

In addition, one study investigated whether uneven ground condition could affect workers' maximum acceptable weights of lift with symmetric freestyle (Wickel \& Reiser, 2008). Participants were required to step on five different slanted grounds $\left(10^{\circ}\right.$ and $20^{\circ}$ facing uphill, $10^{\circ}$ and $20^{\circ}$ facing downhill and flat ground) and lift weight to knuckle height. Participants' whole body kinematics was recorded to examine their sagittal plane joint angles and foot placement relative to the weight. Results indicated that slope did not alter maximum acceptable weights of lift while the participants' foot placement was significantly affected by different ground conditions. Therefore, authors concluded that care should be taken when facing uphill due to the tendency to stand further from the load horizontally and when facing downhill due to increased trunk lean.

\subsection{The anatomy of human spine}

\subsubsection{Spinal column}

To maintain the structural integrity of the lumbar spine, there are several distinct tissues, including bones, fascia, cartilage, ligaments etc. (Chaffin et al., 2006). The vertebral column is defined as the series of vertebrae extending from the base of the skull to the coccyx (i.e. tailbone) 
that forms the supporting axis of the body in vertebrate animals. It encloses and protects the spinal cord and provides a stable attachment for the muscles of the trunk. It consists of 33 vertebrae in total, including 7 cervical vertebrae (C1-C7), 12 thoracic vertebrae (T1-T12), 5 lumbar vertebrae (L1-L5), 5 fused sacral vertebrae (S1-S5) and 4 fused coccygeal vertebrae (tailbone) (Dorland, 2011) (Figure 3). The vertebra in an individual bone in the spine serves as the foundation and mainly provides skeletal support of the body. A functional spinal unit includes two end-to-end vertebrae, an intervertebral disc and associated ligaments (superspinal ligament, interspinal ligament, posterior longitudinal ligament and anterior longitudinal ligament). The vertebra is defined as the bones or segments composing the spinal column, consisting of a cylindrical body and an arch with various processes, and forming a foramen, or opening, through which the spinal cord passes (Figure 4). 

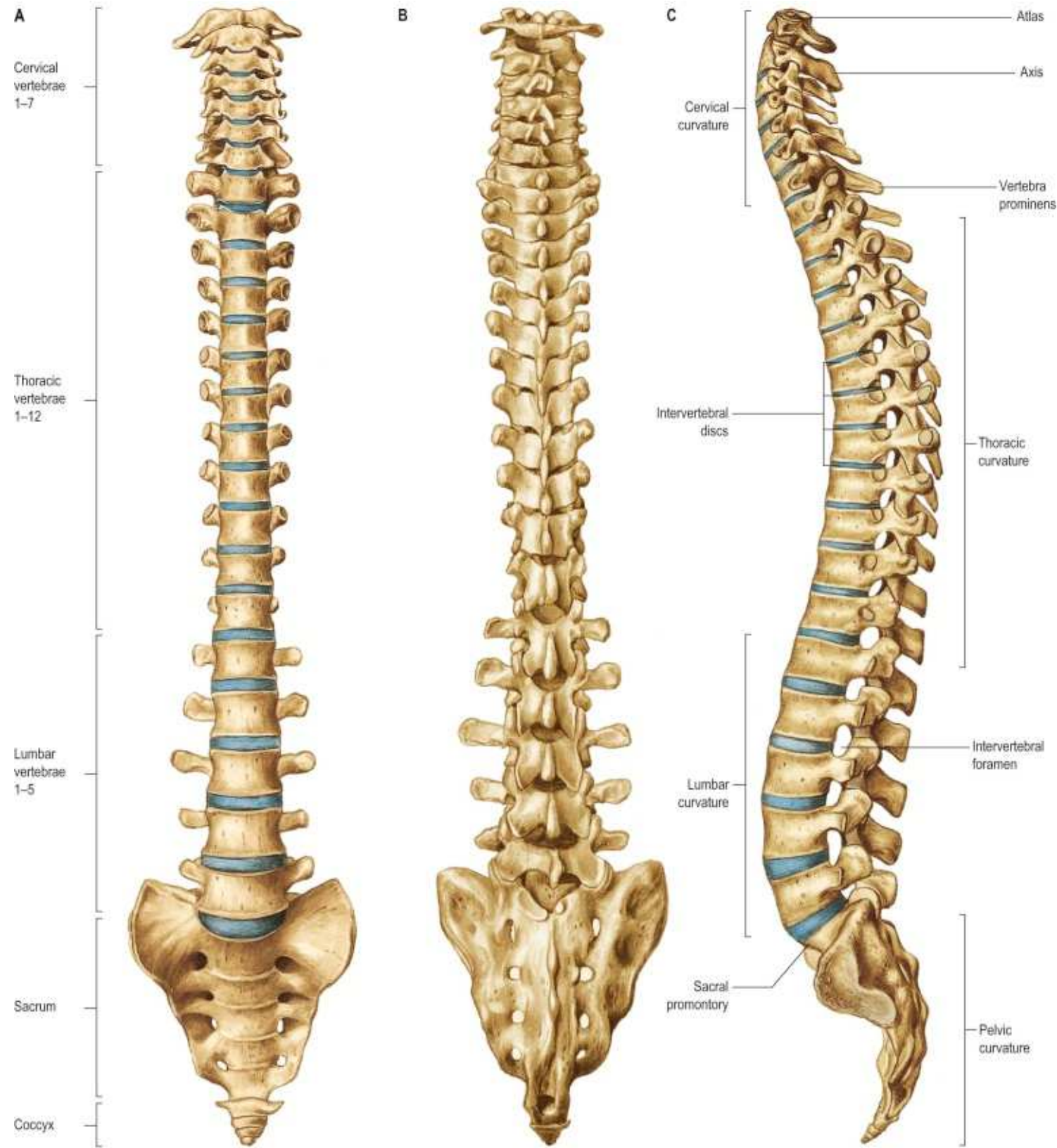

Figure 3: An illustration of human spine (from 2015 Gray's Anatomy) 


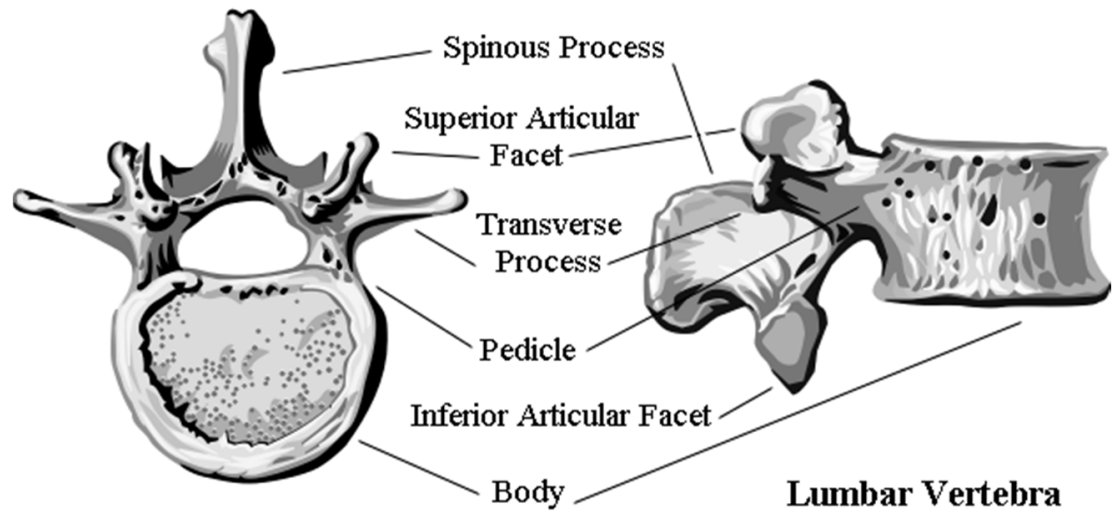

Figure 4: A typical lumbar vertebrae

Between every two vertebrae, there is a tiny structure called the intervertebral disc. The intervertebral disk is a unique articular structure, being part of a load bearing system including intervertebral disks, vertebrae, ligaments and muscles. The intervertebral disc includes the nucleus pulposus, the annulus fibrosis and the cartilaginous end-plates (Figure 5). The main functions of the intervertebral disc are absorbing shocks on the vertebrae, connecting two adjacent vertebrae and providing resistance when the spine rotates.

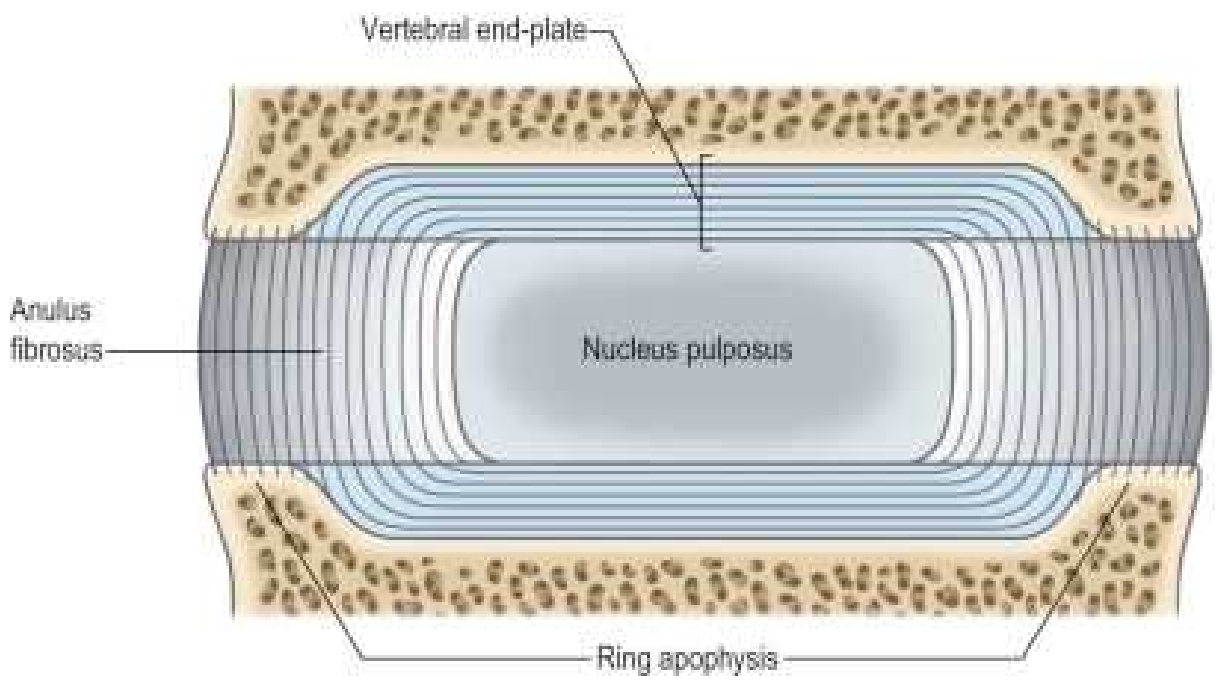

Figure 5: Intervertebral disc illustration (from 2015 Gray's Anatomy) 


\subsubsection{Pelvis}

The pelvis girdle connects the spinal column to the femurs. The pelvis skeleton is formed in the area of the back by the sacrum and coccyx (Figure 6). The sacroiliac joint (SI) is defined as the joint in the bony pelvis between the sacrum and the ilium of the pelvis which are joined by strong ligaments. In humans, the sacrum supports the spine and is supported by ilium on each side (Figure 6). There are five ligaments that connect ilium with sacrum in the SI joints: 1) interosseous SI ligament; 2) anterior SI ligament; 3) posterior SI ligament; 4) sacrotuberous ligament; 5) sacrospinous ligament. In general, the pelvis provides an important connection between upper body and lower body and influences the length and both active muscles and passive tissues during motion.

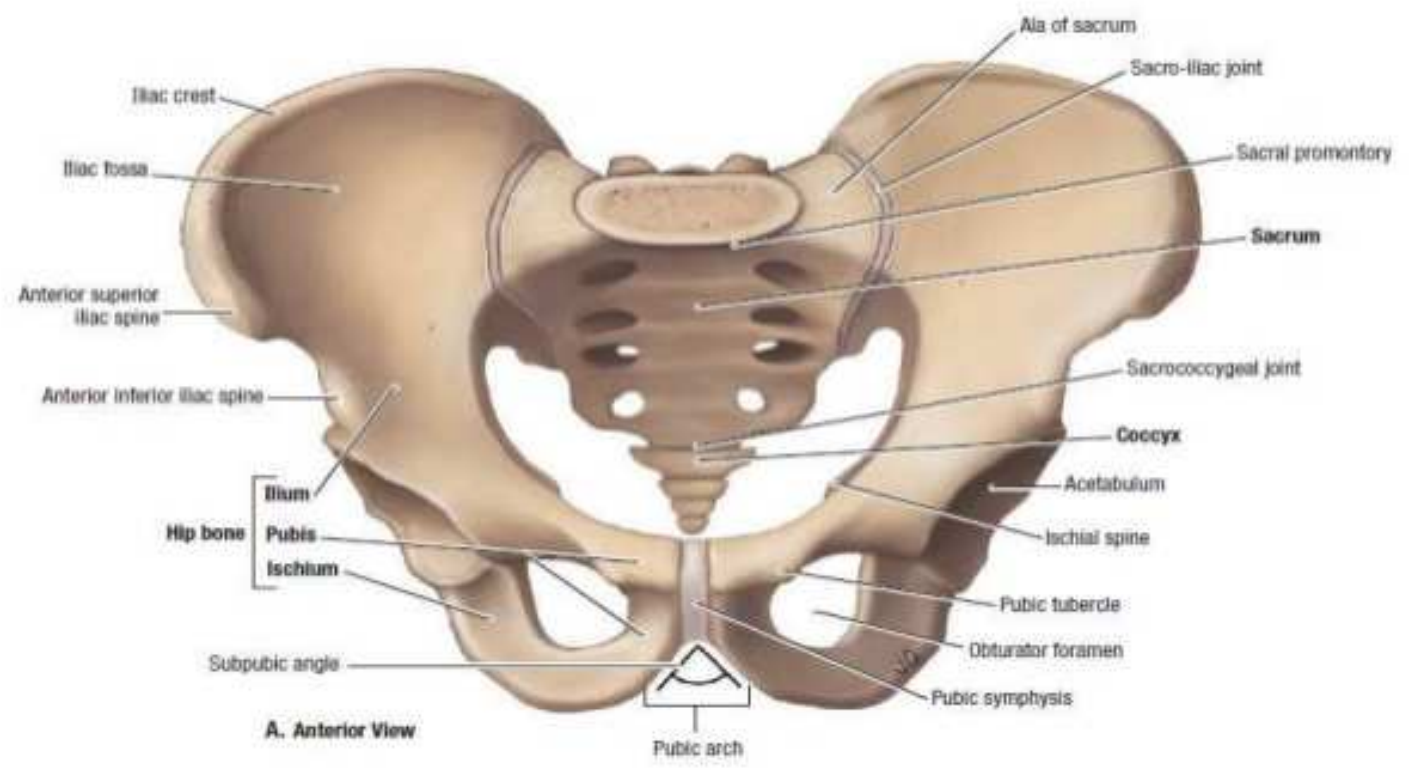

Figure 6: Anterior view of the pelvis 


\subsubsection{Skeletal muscles}

\subsubsection{Lumbar muscles}

There are multiple major muscle groups in the low back region to initiate and maintain the flexion-extension motion and stabilize the spinal column (Bogduk et al., 1992). Major ones include erector spinae, multifidus and latissimus dorsi and psoas major.

To begin with, erector spine is a big muscle mass that lies laterally to the multifidus. It is largely covered by the erector spinae aponeurosis which originates from the dorsal segment of the iliac crest (Bogduk, 1980). It consists of three components, including spinals in the medial column, the longissimus in the intermediate column and the iliocostalis in the lateral column. Erector spinae is the most important lumbar extensor, based on a previous study, it has the largest physiological cross sectional area in the lumbar region (PCSA) (Marras et al., 2001) (Table 3).

Multifidus muscle is the most medial muscle in the low back region and its primary function is to stabilize the spinal column. It arises from the mammillary processes of the sacrum or lumbar vertebrae and is inserted into the spinous process of lumbar vertebra. A previous study was aimed to evaluate the unique design of the multifidus for the lumbar spine stability (Ward et al., 2009). In that study, multifidus muscles were isolated from eight cadaver samples and the following measurements were recorded: mass, sarcomere length, normalized fiber length, physiological cross-sectional area and fiber length-to-muscle length ratio. Analyses results showed that the multifidus muscle has an architectural design and thus its main function is to stabilize the spinal column and generate large force. Besides, the unique position of the muscle allows it to become stronger as the spine bends forward. 
Table 3: PCSA $\left(\mathrm{cm}^{2}\right)$ for each muscle. Bolded cells indicate a significant difference between different side (p-value < 0.05 ) (from Marras et al., 2001)

\begin{tabular}{|c|c|c|}
\hline Muscle & Females & Males \\
\hline R. latissimus dorsi & $13.29(5.0)$ & $21.74(4.2)$ \\
\hline L. latissimus dorsi & $12.01(4.7)$ & $19.44(5.1)$ \\
\hline R. erector spinae & $16.16(3.8)$ & $25.95(4.1)$ \\
\hline L. erector spinae & $16.12(3.4)$ & $26.00(4.2)$ \\
\hline R. rectus abdominis & $6.28(2.1)$ & $9.05(2.3)$ \\
\hline L. rectus abdominis & $6.46(2.3)$ & $9.04(2.3)$ \\
\hline R. external oblique & $7.24(1.1)$ & $10.6(2.0)$ \\
\hline L. external oblique & $6.92(1.1)$ & $10.59(2.2)$ \\
\hline R. internal oblique & $6.18(1.3)$ & $10.26(2.2)$ \\
\hline L. internal oblique & $6.43(1.1)$ & $10.54(2.4)$ \\
\hline R. psoas major & $10.39(1.7)$ & $19.49(3.6)$ \\
\hline L. psoas major & $10.96(1.7)$ & $19.76(2.8)$ \\
\hline R. quadratus lumborum & $2.24(0.4)$ & $5.26(1.6)$ \\
\hline L. quadratus lumborum & $2.64(0.6)$ & $5.42(1.9)$ \\
\hline
\end{tabular}

The latissimus dorsi covers the back of the thorax and has widespread and distant attachments to the thoracic, lumbar and sacral spinous processes and also to the ilium. The function of latissimus dorsi is mainly for initiating the movement of the upper limb and shoulder adduction, not generating force to extend the trunk (Bogduk et al., 1998).

Lastly, the psoas major originates from the lateral surface of the T12 vertebrae and runs down to reach the psoas tendon. The psoas major is designed to provide force to flex the hip and also stabilize the spinal column. However, because of its relative short distance to the center of spinal column (i.e. short moment arm), the moment it can generate is minimal. 


\subsubsection{Abdominal muscles}

Lateral anterior abdominal region has three muscle groups: transversus abdominis $(\operatorname{TrA})$, obliquus internus abdominis (OI) and obliquus externus abdominis (OE) (Figure 7). The OE is the most superficial muscle among the three. It originates from the external surface of ribs $\left(5^{\text {th }}\right.$ to $12^{\text {th }}$ ) and inserts into the anterior abdominal aponeurosis and iliac crest. OE has the middle range of muscle thickness among the three but has the longest fascicles (Urquhart et al., 2005). The function of this muscle includes raise the intra-abdominal pressure and compress the abdominal cavity.

The OI lies deep to the OE and superficial to the TrA, runs perpendicular to the OE activation direction. This muscle originates from anterior two thirds of the iliac crest and lumbodorsal fascia of the low back and inserts into the costal cartilages of ribs ( $8^{\text {th }}$ to $\left.12^{\text {th }}\right)$ and abdominal aponeurosis. OI is designed to support the abdominal wall, flex and rotate the trunk and also raise intra-abdominal pressure.

The TrA lies underneath of the OI and is the innermost of the flat abdominal muscles. It originates from the lumbodorsal fascia of the low back, anterior iliac crest and cartilages of ribs $\left(6^{\text {th }}\right.$ to $\left.12^{\text {th }}\right)$ and inserts to the abdominal aponeurosis, xiphoid process and public symphysis. The TrA is designed to provide compression force on the abodomen. Therefore, its activation is always associated with adjusting intra-abdominal pressure (Cresswell et al., 1992). When contracting the abdominal muscles, the increase of intra-abdominal pressure is also believed to provide stability to the lumbar spine (Cholewicki et al., 1999; Hodges \& Gandevia, 2000). 


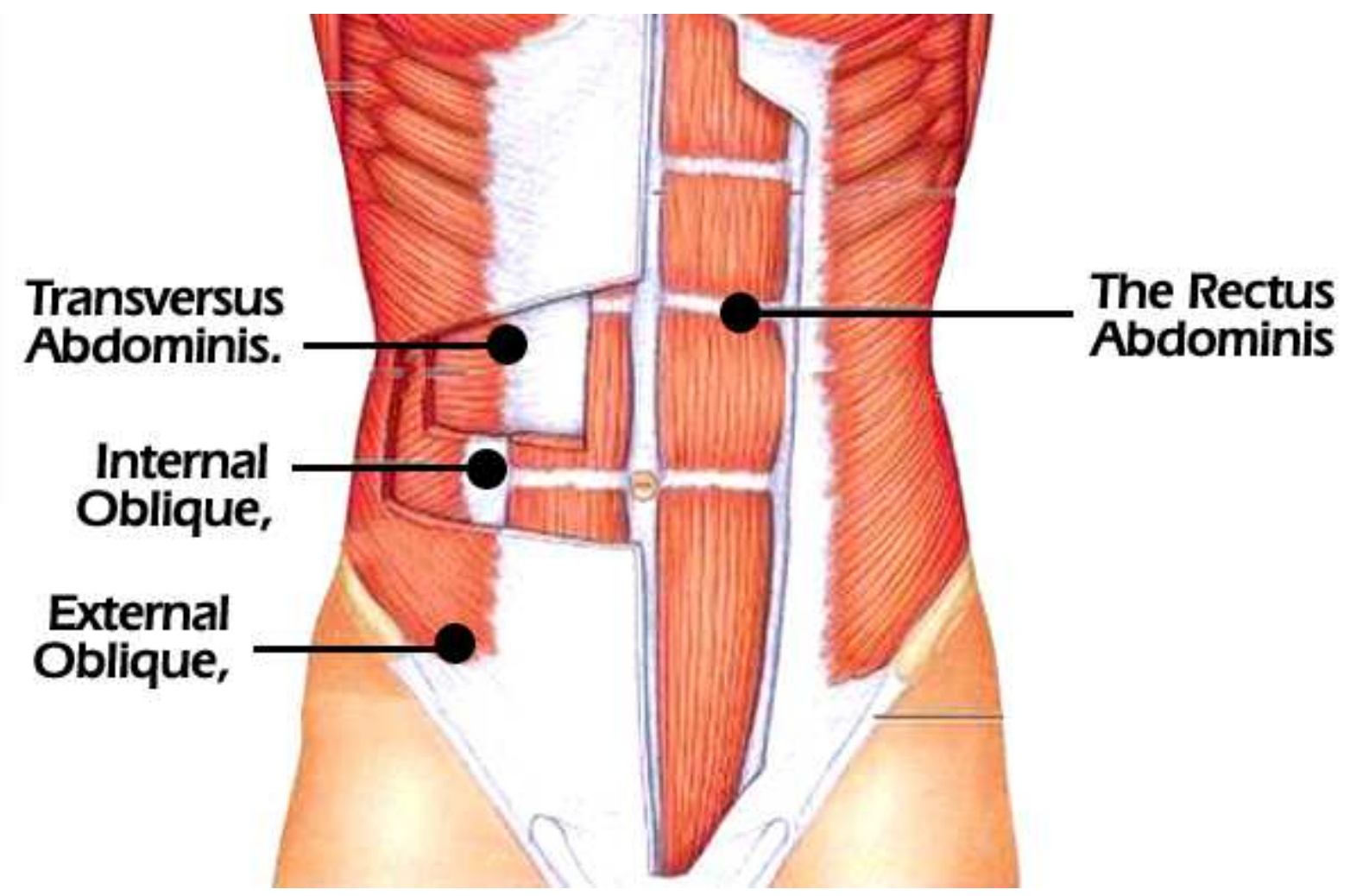

Figure 7: An illustration of transversus abdominis (TrA), obliquus internus abdominis (OI) and obliquus externus abdominis (OE) (photo credit: www.brentbrookbush.com)

\subsubsection{Connective tissues}

Another important component of the spine is the ligament and there are six types of ligaments in the vertebral column: 1) anterior longitudinal ligament which is a relatively strong band spreading along the anterior surface of the vertebral; 2) posterior longitudinal ligament and it lies inside of the vertebral canal, on the posterior side of the vertebral bodies and goes with the membrane tectoria above; 3) ligament flava connects laminae of adjacent vertebrae in the vertebral canal; 4) interspinous ligaments are defined as the ligaments connect the edges of consecutive spinous processes and spread as far as the ligamentum flavum and dorsally to the supraspinous ligament when this ligament is present; 5) supraspinous ligament connects the tips 
of spinous processes from $\mathrm{C} 7$ to L4 level; 6) intertransverse ligaments go between adjacent transverse processes (Figure 8). In general, ligaments are short and wide and include a great percentage of ground substance (Chaffin et al., 2006). The main function of spinal ligaments is to connect adjacent vertebrae and limit the movement of spine. Furthermore, when being stretched, these ligaments could generate considerable amount of passive force that serves to balance the external loads.

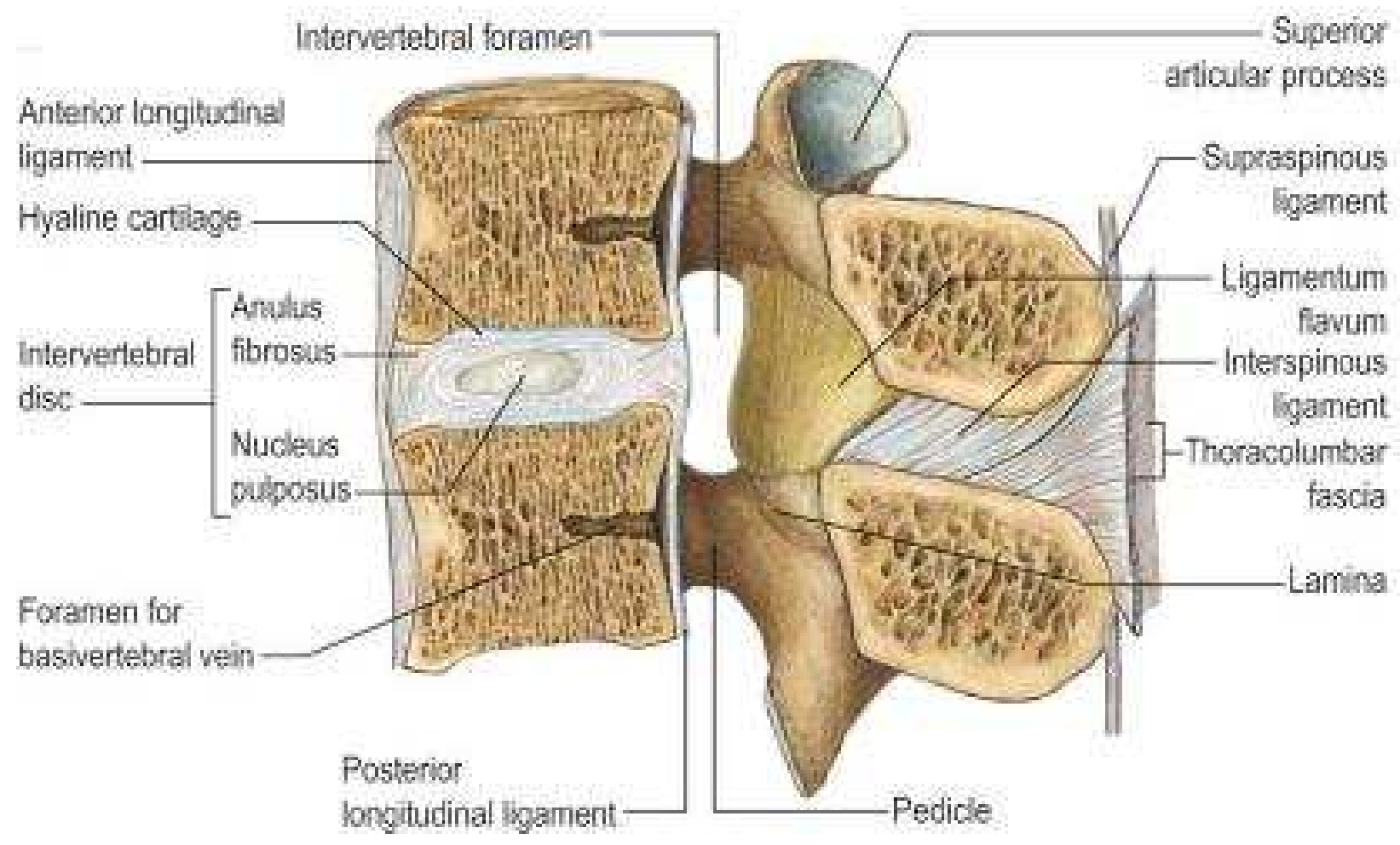

Figure 8: A demonstration of spinal ligaments (from 2015 Gray's Anatomy)

Fascia is a dense connective tissue that covers organs or parts of organs and separates them from each other (Chaffin et al., 2006). The primary fascial layers in the axial and paraxial regions of the trunk include the thoracolumbar fascia and the pelvic fasciae (prevertebral, endothoracic, 
retroperitoneal and posterior part) (Figure 9). The function of fascia is to transfer load from the low back region to the lower extremities and through the pelvis. Previous study also reported that lower extremity muscles coordinate with lumbar muscles through lumbodorsal fascia (Vleeming et al., 1995).

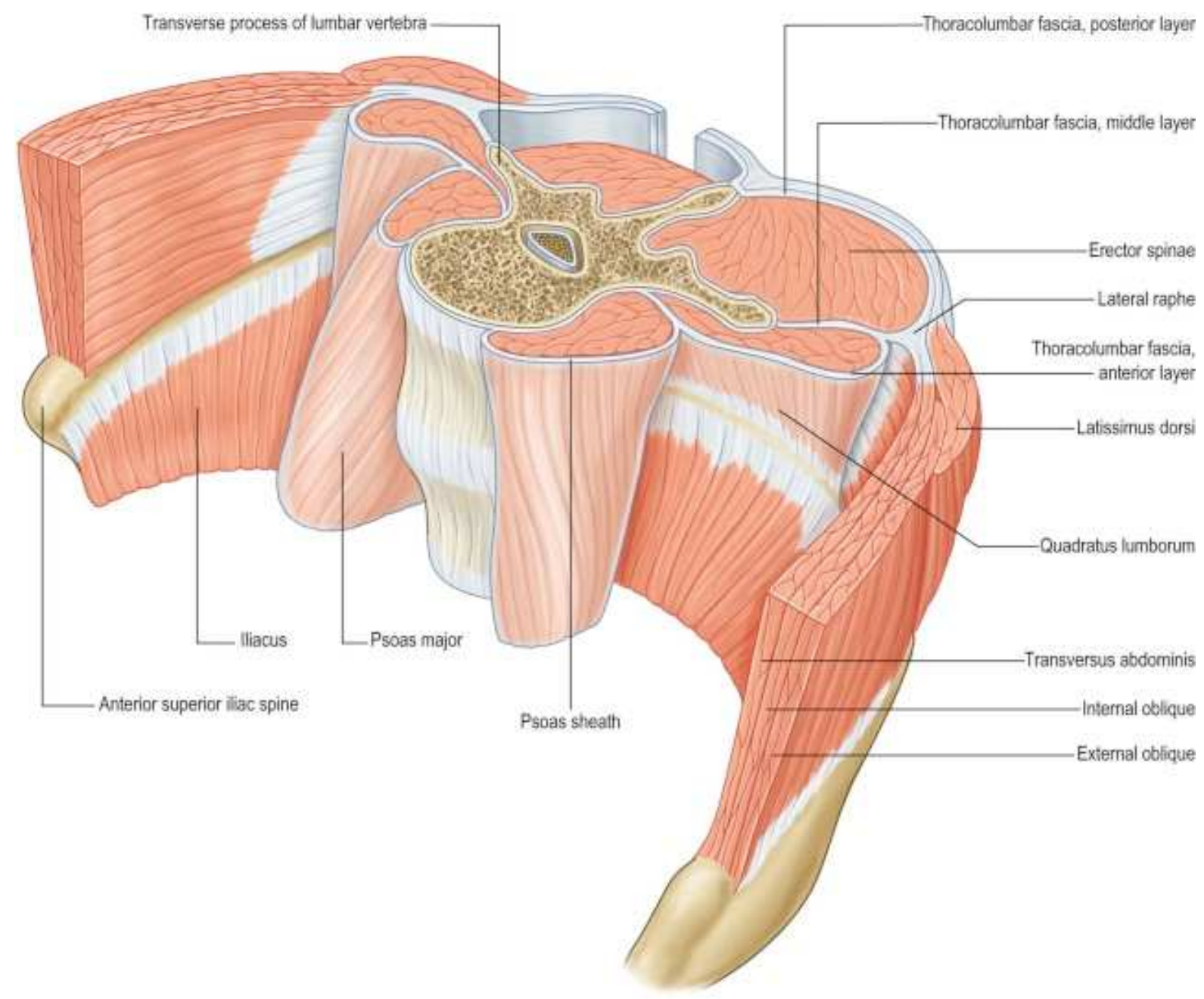

Figure 9: Fascial layers of the posterior abdominal wall (from 2015 Gray's Anatomy) 


\subsection{Trunk flexion and low back pain}

Trunk flexion is commonly seen in occupational tasks such as mining (Gallagher 2008), agriculture (Fathallah 2010) and construction (Boschman et al., 2011). Previous studies have identified both static (e.g. posture holding) and dynamic (e.g. lifting and lowering) trunk flexion as risk factors for the LBP (Liira et al., 1996), especially when prolonged trunk flexion and repetitive trunk bending tasks are involved (BLS, 1999; Muslim et al., 2013; Hoogendoorn et al., 2000). As the trunk flexes forward from the upright standing position, lumbar extensor muscles (erector spinae, multifidus, etc.) need to be activated to counterbalance the gradually increasing external moment. Due to the relatively small moment arms that lumbar extensor muscles have, large amount of loading (compression and shear forces) on the vertebrae and intervertebral discs is induced (Toussaint et al., 1995). Previous literature has shown that excessive loading on the spinal structure could lead to spinal disorder and pain (Marras et al., 2001). Occasionally performed trunk flexion with moderate hand load is acceptable and may not cause immediate severe damage to the spinal structure. However, prolonged or repetitive trunk flexion could generate damages to the spinal structure and lead to LBP eventually (Coenen et al., 2012).

Trunk flexion influences the spinal biomechanical responses mainly via two means: through the unique muscle contraction attributes in the lumbar region (i.e. difference between concentric and eccentric) and through the influence of muscle co-contraction.

\subsubsection{Trunk muscle concentric and eccentric exertions}

Muscle contraction was defined as the process of force generation in the fibers of any class of muscle, by the interaction of myosin head-groups in the thick filaments with actin molecules in 
one of the immediately neighboring thin filaments. Muscle contraction can be classified into three categories based on the alternations in muscle length: concentric contraction (results in shortening muscle length), isometric contraction (muscle length remains unchanged) and eccentric contraction (results in lengthening muscle length) (Figure 10).

Regarding trunk flexion and extension motion, the flexion phase is the eccentric contraction and the degree of overlap between myosin and actin decreases with body motion while the extension phase is the concentric contraction and the overlap between the myosin and actin increases with the body motion. According to a study by Proske and Morgan (Proske \& Morgan, 2001), eccentric contraction has higher risk for causing damage to the tissues since the bonds between myosin and actin are disrupted by external mechanical force during the fiber lengthening motion. In other words, trunk flexion has higher risk level and could generate larger damage compared with trunk extension motion.

Previous studies have reported muscular activation differences between eccentric and concentric contraction. It has been reported that under the same level of force generation condition, eccentric contraction had smaller electromyography level compared to concentric contraction (Huang \& Tohrstensson, 2000). Tesch and colleagues also reported that the ratio of electromyography level and moment is significantly larger in the concentric contraction motion (Tesch et al., 1990). In addition, during the eccentric contraction motion, greater force generation capacity has been observed during the maximum voluntary contraction and the larger force generation might be attributable to the passive force from the stretched passive tissues (McCully \& Faulkner, 1985). 


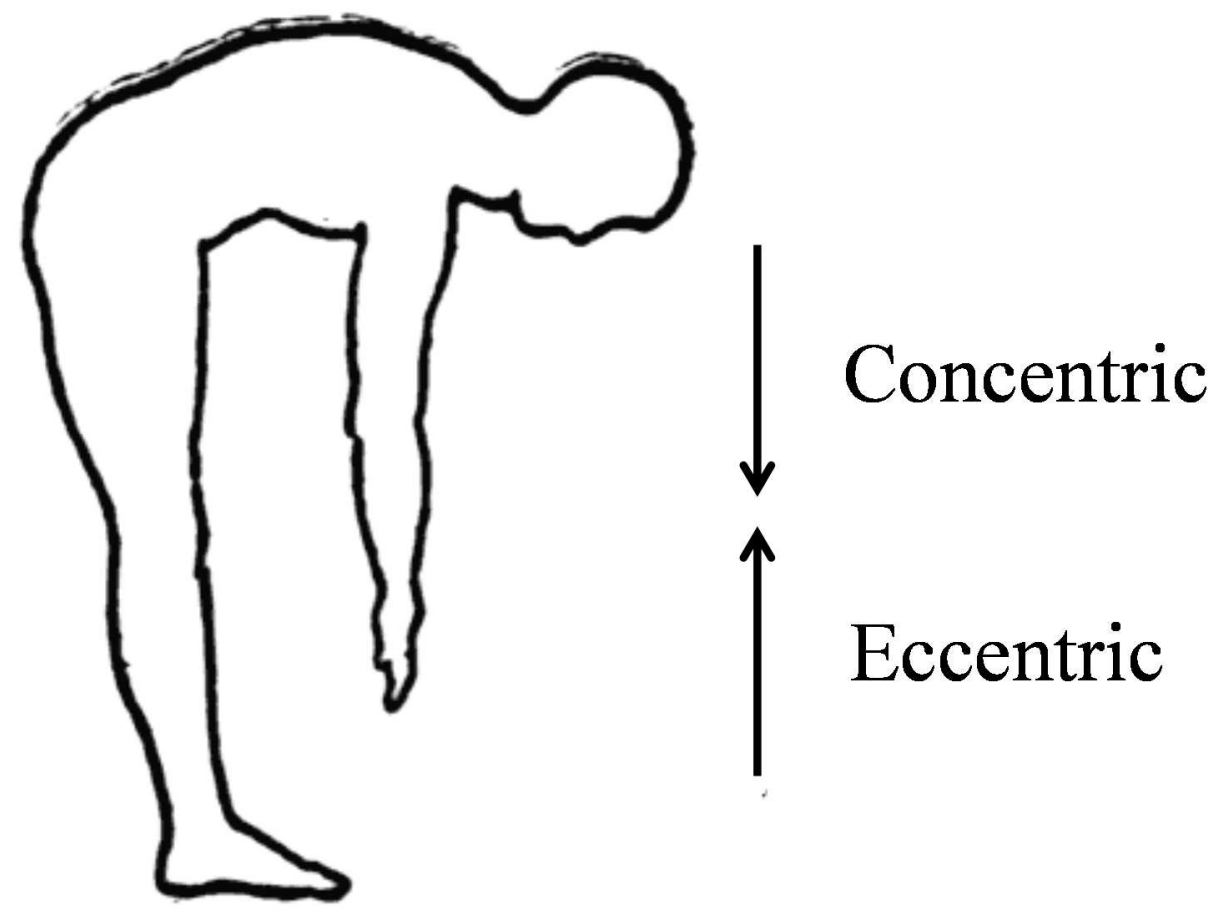

Figure 10: A demonstration of concentric and eccentric muscle contraction for lumbar extensor muscles

\subsubsection{Trunk muscle co-contraction}

Muscle co-contraction is defined as the simultaneous contraction of both the agonist and the antagonist around a joint to hold a stable position. The primary function of antagonistic exertion is not to initiate or maintain any motion since it does not contribute to generating any driving force. As a matter of fact, excessive antagonist muscle contraction could increase the spinal compression load and the risk of spinal damage (Hughes et al., 1995; Marras \& Granata, 1996). In one study, the influence of antagonist muscles co-contraction on spinal loading (i.e. compression force) is evaluated with Karush-Kuhn-Tucker (K-K-T) multipliers. A mathematical model whose function is spinal compression force is used to calculate the influence of cocontraction on spinal compression force. Results indicated that the marginal effect of co- 
contraction on spinal compression force can be 5 times larger than without co-contraction condition.

In another study, Marras and Granata (Marras \& Granata, 1996) investigated the effects of muscle co-contraction on spinal loading during lateral bending motion. To that end, twelve participants were required to lift loads using lateral bending posture at three dynamic velocities and one static posture. Electromyography activities of 10 lumbar extensor and flexor muscles were recorded using surface electromyography sensors while their kinematics performances were also recorded. All data were exported to an EMG-assisted biomechanical model to predicted spinal loading under different experimental conditions. Analyses results showed that muscle cocontraction occurred in all lateral bending trials and it could increase spinal loading up to $25 \%$ compared to without co-contraction model prediction. In addition, spinal anterior-posterior shear and lateral shear force also increased.

Many previous studies have shown that the function of lumbar muscle co-contraction is to enhance spinal stability and protect the spine under abnormal low back conditions. One study evaluated the effects of muscle co-contraction on spinal stability during lifting exertions (Granata \& Orishimo, 2001). In that study, a two dimensional spinal stability biomechanical model (lumbar muscle electromyography data and whole body kinematics were set as model input) was used to evaluate the relation of muscle co-contraction and lifting height and external load level. Results indicated the improvement of trunk stiffness by the recruitment of antagonistic cocontraction. 
More recently, Lee et al conducted a study to evaluate the effects of muscle co-contraction on trunk stiffness (Lee et al., 2006). In that study, pseudo-random force disturbances were applied during trunk extension to generate trunk displacements in varied conditions and electromyography from eight lumbar muscles were recorded as a baseline measure of cocontraction. Trunk stiffness was determined from impulse response functions (IRFs) analyses. Results demonstrated a $37.8 \%$ increase in terms of trunk stiffness from the minimal to maximal co-contraction conditions and these results supported the assumption that antagonist muscle exertion could be attributable to stabilize the spinal system.

Finally, Granata and Marras investigated the cost-benefit relationship of increasing muscle cocontraction and spinal stability (Granata \& Marras, 2000). A biomechanical model was developed to estimate lumbar stability and spinal load and analyses results showed a $34 \%$ to $64 \%$ increase in terms of spinal stability and a $12 \%$ to $18 \%$ increase in terms of spinal compression load with higher muscle co-contraction. In conclusion, the central nervous system (CNS) may use muscle co-contraction as a means to balance between spinal loading and stability performance and achieve the system optimization status.

\subsection{Lumbar muscle flexion-relaxation phenomenon}

The human lumbar spine is a structure with high degree of complexity. In general, lumbar tissues can be divided into active tissues and passive tissues. Active lumbar tissues refer to the contractive component of lumbar muscles; passive lumbar tissues include ligaments, fascia, vertebrae, discs, the passive component of lumbar muscles and all other tissues that do not

voluntarily generate force. Both active and passive lumbar tissues act in concert to initiate, 
maintain or stop trunk motions. Early studies discovered that during trunk bending, lumbar extensor muscles would suddenly cease action when reaching close to full trunk bending posture (Floyd \& Silver, 1955). This phenomenon illustrated the close interaction between active and passive lumbar tissues and was later referred as flexion relaxation phenomenon (FRP). More recently, FRP was frequently investigated to enhance our understanding in the lumbar tissue neuromuscular behaviors and the synergy between active and passive lumbar tissues.

\subsubsection{The underlying mechanism of lumbar muscle FRP}

FRP has been described as a synergistic load sharing mechanism between active muscles and passive viscoelastic tissues in low back region during the trunk flexion and extension motion (Schultz et al., 1985). As the trunk bends deeper, larger external moment on the L5/S1 joint (caused by the upper body) is expected to increase since moment arm is increasing. Therefore, it would be intuitive to expect that the low back muscle activity level will increase gradually to compensate for the increased external moment. However, passive tissues are stretched at the same time and at some point, stretching force from the passive tissue will be enough to counterbalance the external torque and finally result in no muscle activity in the paraspinal muscles (i.e. EMG off point) (Figure 11). During trunk extension phase, the low back extensor muscle activity reappears at certain point (i.e. EMG on point) and remains until full extension posture to generate active extension moment. The point of EMG off and EMG on is normally described in lumbar flexion angle or trunk inclination angle since FRP is directly affected by lumbar curvature. A number of researches have shown the presence of the FRP in asymptomatic participants (Solomonow et al., 2003; Mathieu \& Fortin, 2000). One study recorded EMG activity at the L3 and L4 level over the erector spinae musculature during upright trunk flexion 
tasks and calculated trunk flexion angle with 49 asymptomatic participants. Results showed that FRP was observed among all participants during trunk flexion motion with the average EMG off lumbar angle ranging from $46^{\circ}$ to $50^{\circ}$ of trunk flexion (Solomonow et al., 2003).

Other research has been performed to evaluate load sharing between spinal tissues in the trunk flexion posture (Schultz et al., 1985). Participants were required to conduct trunk flexion task with varied loading conditions which their lumbar extensors' EMG were recorded and used to estimate loading on the lumbar trunk structures. An optimization method was used to calculate the net support reaction needed to counterbalance the external load at the L3 level followed by estimation of muscle activation level that could supply the net reaction. Results showed that during trunk flexion motion, EMG signals of the erector spinae (i.e. lumbar extensor muscle) were significantly smaller than quiet static standing posture despite the need to generate posterior tissue tensions equivalent to erector spinae contractions over $700 \mathrm{~N}$ at $40^{\circ}$ or more of flexion to keep equilibrium. Therefore, passive tissue forces are required to maintain the load requirements in the trunk flexion posture. 


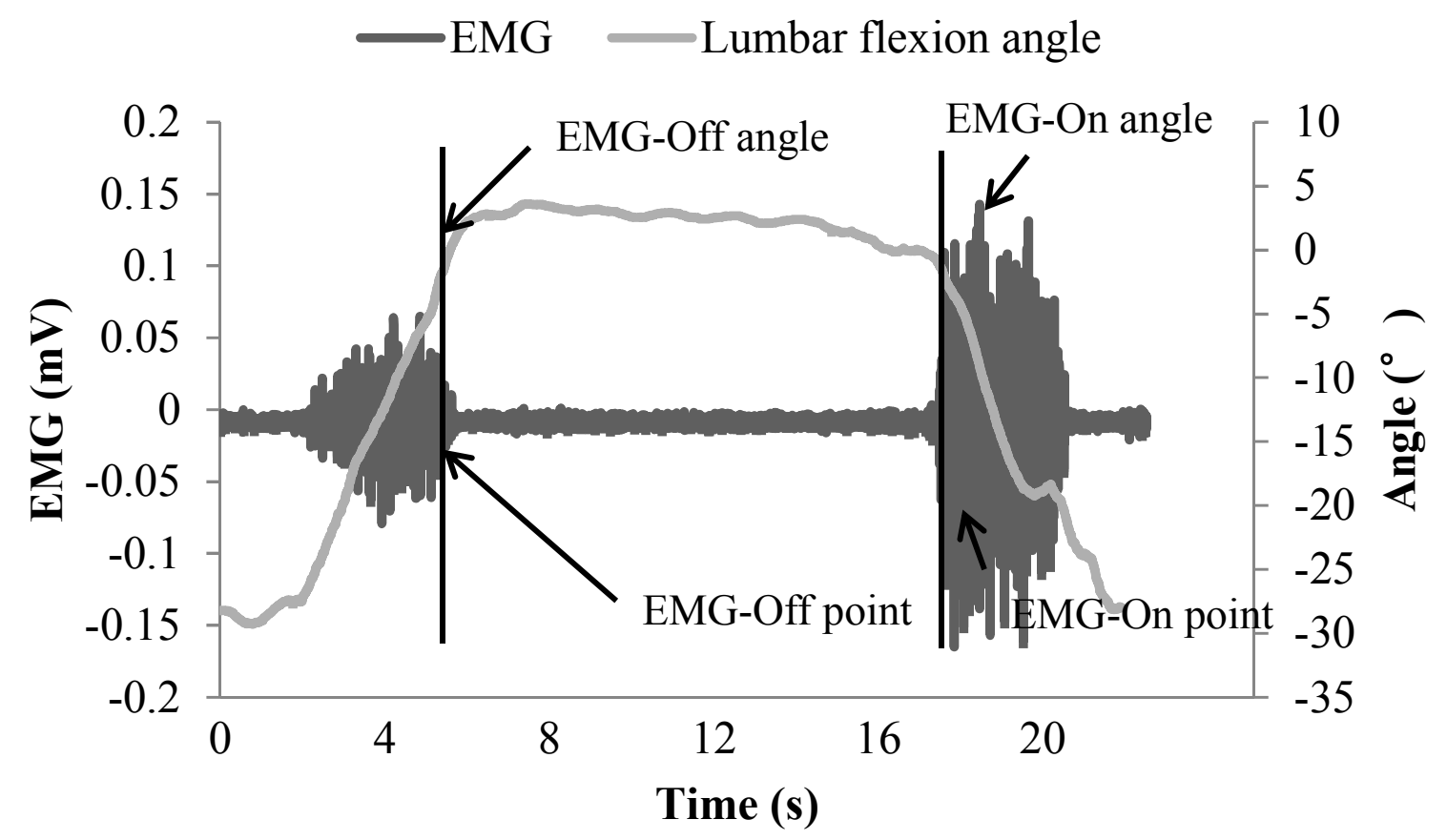

Figure 11: A demonstration of the "EMG-off" and "EMG-on" point of a back muscle and the corresponding lumbar flexion angle

Another study conducted by McGill and Kippers was aimed to investigate the loads on individual tissue during deep trunk flexion motion (McGill \& Kippers, 1994). An anatomically detailed model of the lumbar spine (vertebral displacement and EMG signals were used as model input) was used to estimate individual muscle and passive tissue force profile. Analyses results indicated that in full trunk flexion posture, loading of lumbar passive tissues (e.g. interspinous and supraspinous ligaments, posterior annulus of the intervertebral disk) was found to be high compared to their failure tolerances. Therefore, deep trunk flexion increases the failure risk of lumbar posterior passive tissues. In addition, results also showed even though EMG activity level of many lumbar extensor muscles remained low in full flexion posture, forces were still generated in the lumbar extensor muscles since those muscles were stretched far beyond their resting length, enabling the muscles to generate forces in the absence of neural activation. 
Dolan and colleagues investigated the lumbar passive tissue contribution in weight lifting task (Dolan et al., 1994). In the study, 149 participants were recruited and the experimental tasks included pulling upward with steadily increasing force on a load cell which was amounted on the floor while EMG signals from the erector spinae at the L3 and T10 levels were recorded. Data from the load cell was used to estimate the extensor moment and was plotted against the rectified EMG data. A linear relationship could be found which indicated that flexion moment was resisted by passive tissues (with an extra intercept on the extensor moment axis). Analysis of the dependence of the extensor moment axis was confirmed with follow up analyses and results showed that 3 to 4 fold increases in the extensor moment axis occurred for the full flexion posture from the lordotic posture which was in agreement of the findings from McGill and Kippers' study.

One study was aimed to explore the biomechanical consequences of the FRP during trunk flexion (Gupta, 2001). Author measured the EMG signal differences by inducing abnormal combinations of hip and trunk movement: participants were asked to bend forward again a wall at backward which restricted the posterior movement of the pelvis and limited the pelvic component of trunk flexion. Furthermore, extra weights were added around participants' iliac crest and hands. EMG signals from the erector spinae (at L3 level), rectus abdominis, hamstrings and gluteus maximus were recorded with surface EMG electrodes. Participants' kinematics performances were also recorded using a video camera. Results demonstrated that FRP occurred in all participants at $57 \%$ and $84 \%$ of mean maximum hip and vertebral flexion, respectively. Strong EMG activity was observed among the hamstring muscles. When forward bending was performed against the wall, the FRP occurred much earlier $(28 \%$ and $75 \%$ of the hip and vertebral maximum flexion value). The extra weight placed either anteriorly or posteriorly was 
found to increase the tensile torque about the spine and required the lumbar extensors to remain active through a longer period until the passive moment generated by the passive tissues is enough to counterbalance the external moment.

It has been reported that the reflex response is controlled with trunk flexion and extension and controls low back muscle recruitment (Granata \& Rogers, 2005). However, instead of muscular activation, FRP was observed (i.e. muscle was silenced). Dyhre-Poulsen and Krogsgaard concluded that the inhibitory reflexes could be initiated in the ligament muscular reflex to prevent extreme stress on the joint in addition to the excitatory reflex from the ligaments to muscles (Dyhre-Poulsen \& Krogsgaard, 2000). What is more, one study showed that even though low back muscle activity was at minimal level at deep trunk flexion posture, the trunk stiffness was improved due to the tension of the passive tissue stretching effects (Granata \& Rogers, 2005).

\subsubsection{Factors that could influence lumbar muscle FRP}

Previous studies have reported that lumbar extensor muscle FRP (and the underlining lumbar tissue synergy) could be affected by a number of factors. First of all, one study was conducted to

determine if varied speed and loading conditions during trunk flexion/extension motion could affect the occurrence and disappearance of the erector spinae muscles (Sarti et al., 2001). A total of twenty two participants performed multiple trunk flexion-extension tasks with varied speed and hand load while the motion of the lumbar spine and electromyography of erector spinae were recorded. The percentage of the maximum lumbar spine flexion at the EMG on and off moment and relative lumbar spine motion time during experimental trials were calculated and compared 
between different conditions. Statistical analyses indicated that the increase in speed of motion considerably increased the relative lumbar flexion time and reduced the relative lumbar extension time. Furthermore, increase of speed significantly delayed the EMG off point in the range of motion. However, load had no effect on FRP in that study.

Ning et al. evaluated the influences of asymmetry on the flexion relaxation phenomenon during trunk flexion task (Ning et al., 2011). Twelve participants performed deep trunk flexionextension motion under three different asymmetric conditions (sagittally symmetric, $15^{\circ}$ and $30^{\circ}$ from the mid-sagittal plane). The electromyographic activity from the paraspinals at the L3 and L4 levels and trunk kinematics from the magnetic field based motion sensors over the C7, T12 and S1 vertebrae were recorded. Results indicated that flexion relaxation was observed in the muscles contralateral to the direction of the asymmetric trunk flexion motion. The response of the ipsilateral extensor musculature was significantly different and the FRP occurrence rate was much lower. Increasing asymmetry from $0^{\circ}$ to $30^{\circ}$ led to a $10 \%$ reduction in the maximum lumbar flexion. Lumbar flexion angles necessary to achieve flexion relaxation in the contralateral muscles also decreased.

A study was conducted to identify the effects of erector spinae muscle fatigue and spine loading on flexion relaxation onset and off performance in healthy individuals during a trunk flexion and extension task (Descarreaux et al., 2008). In this study, twenty healthy participants were recruited and performed three trunk flexion trials in four different experimental conditions (no fatigue without load, no fatigue with load, fatigue without load and fatigue with load). Sorenson protocol was used to generate lumbar extensor muscle fatigue and electromyographic power spectral analysis was performed the muscular fatigue was adequate. Furthermore, surface EMG 
of the erector spinae at L2 and L5 levels and trunk and pelvis rotation angles were recorded during flexion extension tasks. Repeated measures ANOVA analyses were performed to evaluate the influences of independent variables on the EMG off and EMG on trunk angles. Results showed that onset of myoelectric silence during the flexion motion appeared significantly earlier after the fatigue task. In addition, the silence of myoelectric off was observed later during the trunk flexion task after the fatigue protocol. When there was extra loading in hand during the trunk flexion task, erector spinae was activated during the whole trial and no FRP was observed in experimental trials. To make a conclusion, the results of this study showed that the presence of fatigue of the erector spinae modifies the FRP. Lumbar extensor muscle fatigue seems to induce a shift in load sharing towards passive stabilizing structures. The loss of muscle contribution together with or without laxity in the viscoelastic tissues may have a considerable impact on post fatigue stability.

A study was conducted by Shin and colleagues to identify the occurrence of passive tissue creep development in the low back during static upper body deep flexion that resembled an above ground occupational posture (Shin et al., 2009). To this end, twenty participants were recruited and performed submaximal isometric trunk extension exertions and an isokinetic trunk flexion before and immediately after a lumbar flexion protocol (5 minutes in total) which the trunk sagittal flexion angle and the electromyography activities of the back extensor muscles were recorded at the same time. Next, lumbar extensor muscle activity level, median power frequency of EMG associated with the static flexion, changes in the flexion relaxation angle and the maximum flexion angle were calculated and compared. Results of statistical analyses indicated that flexion relaxation onset angle in isokinetic flexion and EMG amplitude of isometric extension was considerably greater after the static flexion protocol which showed creep of spinal 
tissues in static flexion. Median power frequency of lumbar erector spinae EMG during isometric extension was significantly lower after static flexion, suggesting that the development of lumbar extensor muscle fatigue. Also, consistent but low level of EMG was observed together with sporadic muscle spasms during the static flexion period. In conclusion, fatigue of low back extensor muscles may occur in static flexion due to prolonged passive stretching of the muscles. Low back extensor muscles are required to generate more active forces in weight holding or lifting after static flexion to compensate for the reduced contribution of creep deformed passive tissues in maintaining spinal stability and the posture. The degraded force generating capacity of the fatigued muscles can be a high risk factor for low back disorders.

One study was targeted to evaluate FRP in chronic LBP patients relative (Alschuler et al., 2009). EMG data were collected from seventy-six LBP patients in maximum voluntary flexion and extension conditions. Results demonstrated that the ratio of maximum EMG during flexion to average EMG during maximum voluntary flexion had the highest association with their musculoskeletal and clinical status and LBP patients showed significantly different pattern compared with their healthy counterparts.

Gender could also influence the occurrence of FRP (Solomonow et al., 2003). Male and female participants performed three groups of trunk flexion-extension before and immediately after a 10-minute period of static lumbar flexion protocol. The EMG data from the erector spinae were recorded surface electromyographic sensors while the lumbar flexion angle was also recorded. FRP off and FRP on angles were compared among different conditions. Results indicated that the erector spinae were activated longer (had larger EMG off and EMG on angles) during the trunk flexion-extension task after the static flexion protocol. Females showed much more 
noticeable changes than male participants. The effects of gender on the passive tissue creep had been reported previous to that paper (McGill \& Brown, 1992). As a result, female participants demonstrated larger increase in lumbar flexion after the flexion protocol. Larger creep of the viscoelastic structures indicated that the active muscles needed to remain active longer in flexion phase and initiate activation earlier in extension phase.

Previous studies have indicated that changes in stance posture could directly influence trunk kinematics and potentially change the lumbar tissue synergy. One study was conducted to investigate the effects of stance width and foot posture on the lumbar muscle relaxation responses during trunk flexion motion (Hu et al., 2013). In that study, participants were required to perform trunk flexion task using three different stance width and two different foot postures (toe-forward or toe-out) (Figure 12). Lumbar muscle EMG was collected from the erector spinae and multifidus; meanwhile three magnetic motion sensors were placed over the S1, T12 and C7 vertebrae to track lumbar and trunk kinematics. The lumbar angle at which muscle activity diminished to a near resting level was recorded. Analyses results indicated that for the erector spinae the flexion relaxation lumbar angle reduced $1.3^{\circ}$ with the increase of stance width. When changed from 'toe-forward' to 'toe-out' foot posture, the flexion relaxation lumbar angle reduced $1.4^{\circ}$ and $1.1^{\circ}$ for the erector spinae and multifidus respectively. These results suggest that it is necessary to control and maintain constant lower extremity posture during the assessment of the lumbar muscles and motion segments; especially when lumbar muscle FRP is used as an indicator and the spine biomechanical response.

Finally, EMG off (FRP initiation moment) and EMG on (FRP cessation moment) and the lumbar flexion angle at such moments are the most commonly used indexes to evaluate FRP 
performance in previous studies. Different algorithms of processing and calculating these indexes can also significantly affect how we interpret FRP. Many studies have been done to develop computer aid algorithms for obtaining FRP indexes and the methods being used are summarized in Table 4. In general, these algorithms include fixed components: 1) certain data smoothing methods are required to generate the muscle activation profile; 2) certain filters are required to remove electronic and background high and low frequency noises; 3) EMG on and off moment determination algorithm. One method being used was a reference based technique. To be specific, FRP will be determined to occur if EMG magnitude dropped to less than certain percentage of MVC value (usually $3 \%$ or $5 \%$ ). The potential problem regarding this method is that MVC is not obtainable from LBP patients and the reference measurement creates considerable variances between trials (Mathiassen et al., 1995). An alternative method is using submaximal reference (50\% of MVC or using EMG magnitude at full flexion as reference). However, this method also has the problem of high intra-participant variance. In addition, in certain conditions such as muscle fatigue or passive tissue elongation, muscular spasm will significantly affect the accuracy and reliability of this algorithm.

To compare the reliability and accuracy of different FRP determination algorithms, one study was conducted by Jin and colleagues (Jin et al., 2012). In that study, six smoothing techniques were tested (no smoothing, moving average, moving standard deviation, Butterworth low pass filter at $0.5 \mathrm{~Hz}, 5 \mathrm{~Hz}$ and $50 \mathrm{~Hz}$ ) while four FRP determination thresholds were tested (EMG less than 3\% of MVC, EMG less than 5\% of MVC, EMG less than 2 times of fully relax EMG and EMG less than 3 times of fully relax EMG).Predictions from those algorithms were compared with the gold standard of expert determined EMG off and EMG on points. The results showed that the " 3 times of fully relax EMG" may generate the most reliable predictions. 
Table 4: Methods for defining onset and cessation of FRP (Jin et al., 2012)

\begin{tabular}{c|c|c|c}
\hline Authors & Threshold & Signal processing & Method \\
\hline $\begin{array}{c}\text { McGill and Kippers } \\
(1994)\end{array}$ & $3 \%$ of MVC & $\begin{array}{c}\text { Low pass filtered at } \\
2 \mathrm{~Hz}\end{array}$ & Reference-based \\
\hline Gupta (2001) & Abrupt changes & N/A & Visual inspection \\
\hline Sarti et al. (2001) & Abrupt changes & $\begin{array}{c}100 \text { ms moving } \\
\text { average }\end{array}$ & Visual inspection \\
\hline Dickey et al. (2003) & $1 \%$ MVC & $\begin{array}{c}\text { Low pass filter at 6 } \\
\text { Hz; Down-sampled } \\
\text { to 20.5 Hz }\end{array}$ & Visual inspection \\
\hline $\begin{array}{c}\text { Solomonow et al., } \\
(2003)\end{array}$ & N/A & $\begin{array}{c}100 \text { ms moving } \\
\text { average }\end{array}$ & Visual inspection \\
\hline $\begin{array}{c}\text { Olson et al. (2004) } \\
\text { during extension }\end{array}$ & $\begin{array}{c}\text { Smoothed at 0,5 Hz } \\
\text { N/A }\end{array}$ & Reference-based \\
\hline $\begin{array}{c}\text { Olson et al. (2006) } \\
\text { (2008) }\end{array}$ & N/A & $\begin{array}{c}10-450 \text { Hz bandpass } \\
\text { filtered }\end{array}$ & Visual inspection \\
\hline $\begin{array}{c}\text { Descarreaux et al. } \\
\text { Shin et al. (2009) }\end{array}$ & $3 \%$ of MVC & $\begin{array}{c}\text { Low pass filtered at } \\
3 \text { hz }\end{array}$ & Reference-based \\
\hline
\end{tabular}
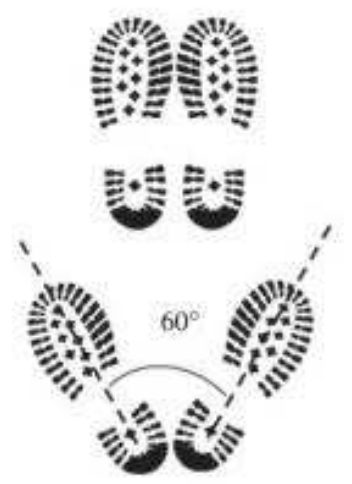
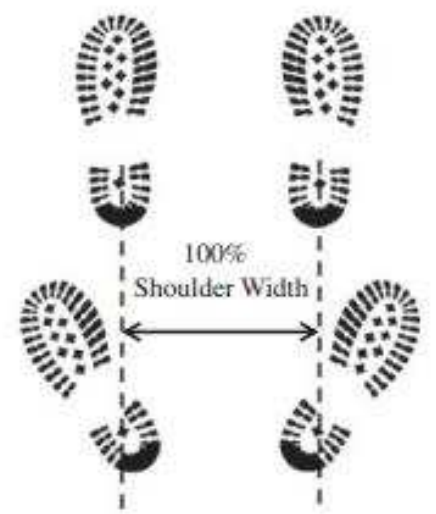
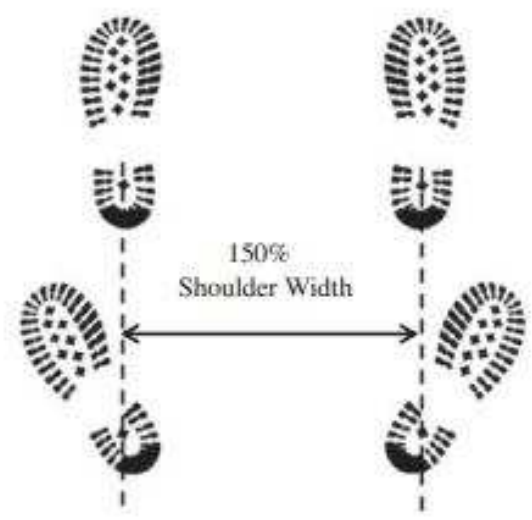

Figure 12: Demonstration of the six different stance conditions (Hu et al., 2014) 


\subsection{Spinal stability}

Stability is one of the most important characters of any system and regarding the spine, stability is critical for the spine to support body motion, undertake load and avoid injury. Human postural control is achieved through combined inputs from the vestibular, visual and proprioceptive systems and those inputs are manipulated and organized by the central nervous system (CNS). The ability to avoid undesirable responses triggered by internal or external perturbations is known to be an important property of the human postural control system (Gurfinkel et al., 1995). By adjusting the environment that affects these systems their function to balance control can be studied. For instance, vestibular input can be altered by changing of the head angle. Also, proprioception can be challenged by changing the support surface configuration (Hlavacka et al., 1996).

\subsubsection{Stability and robustness}

Two important aspects regarding the spinal balance are stability and robustness. These two concepts can be explained using an example of putting a ball on a surface (adapted from Reeves et al., 2007). The shape of the surface will determine if the ball is stable of not. In Figure 13 (a), even though the current status of the ball is stable, any external perturbation will interrupt this equilibrium the ball will roll away. On the other hand, in Figure 13 (b), external perturbation will initiate the motion of the ball, but eventually the ball will return to its initial position thus the status of the ball remains stable in the long run. 


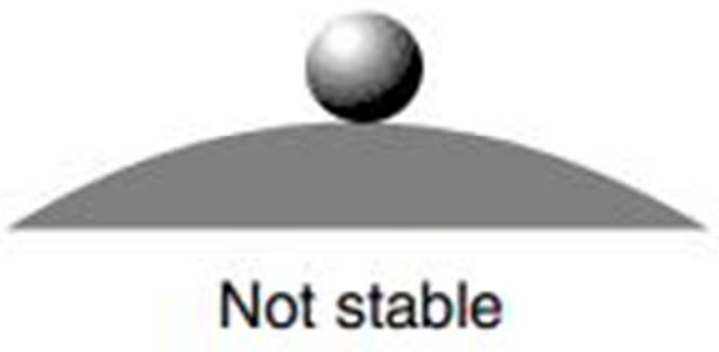

(a)

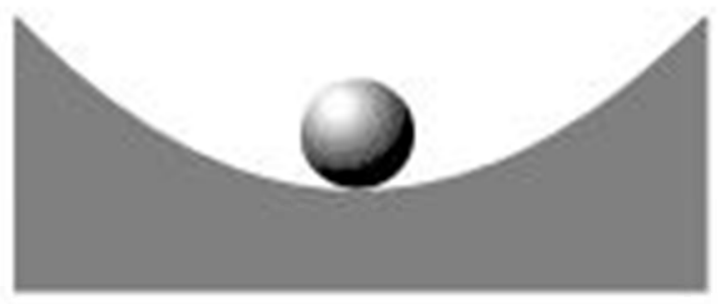

\section{Stable}

(b)

Figure 13: Examples to demonstrate the concept of stability (Reeves et al.,2007)

Robustness of the balance control system is how well the system can cope with the uncertainties and disturbances. A good performance system needs to have relative good robustness (can cope with perturbation in different magnitudes). The idea of robustness and also be illustrated using the surface and ball example. In Figure 14 (a), the slope of the surface is larger than that of in Figure 14 (b), therefore, the ball in the first condition can return to the undisturbed position much faster and thus has a better robustness performance. In the spine system, the slope of the wall in the example is the trunk stiffness and increased stiffness indicates steeper surface in the example. 


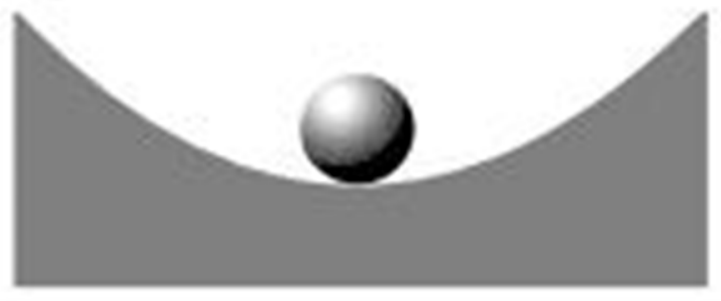

Good robustness

(a)

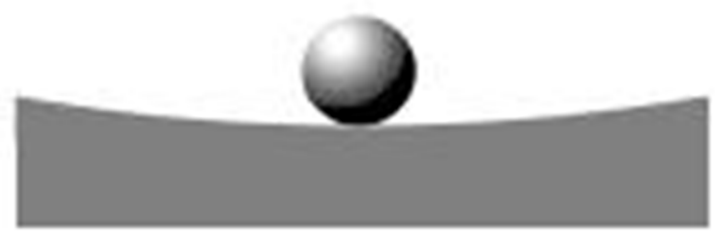

Bad robustness

(b)

Figure 14: Examples to demonstrate the concept of robustness (Reeves et al.,2007)

\subsubsection{Feedback control in the spinal balance control system}

The feedback controller for the spine includes intrinsic properties of trunk muscles (muscle stiffness, damping, etc.), intrinsic properties of intervertebral joints (joint stiffness, damping, etc.) and the CNS which can control both reflexive and voluntary muscle activation. CNS can use different feedback pathways to stabilize the spine depending on the magnitude of the perturbation. For example, trunk muscle co-contraction can increase muscle stiffness and increase the contribution of the intrinsic system properties. On the other hand, in situations that need to maintain for longer periods, CNS could adjust the weight of the reflexive pathways since metabolically speaking reflexive pathway has better effectiveness than muscle co-contraction method. For each particular task, CNS is responsible for finding the optimal solution and maximizing the system's performance. 


\subsubsection{Factors influencing spinal stability}

Many uncontrollable factors can contribute to the degradation of the balance control system such as decreased performance of the sensory-motor system or musculoskeletal disorders. The stability of spine can be affected from its three sub-systems: 1) the active musculoskeletal system; 2) the passive musculoskeletal subsystem and 3) neural subsystem. Micro-damage in any of the three components can influence the performance of the spine significantly.

Previous study has shown that spine proprioception can be affected by external factors including fatigue (i.e. the ability to sense a change in terms of lumbar position) (Taimela et al., 1999). To be specific, participants were asked to sit on a special trunk rotation unit and test their ability to sense a change in lumbar position both before and after a fatiguing protocol. Results showed that lumbar fatigue influences the ability to sense lumbar posture changes in both LBP patients and control groups (p-value $<0.001)$. Another study by Rogers and Granata was aimed to test the effects of prolonged stooping on the paraspinal muscle reflexes (Rogers \& Granata, 2006). Participants performed static flexion relaxation tasks both before and immediately flexion relaxation protocol. Paraspinal muscle reflexes were recorded and quantified using systems identification analysis method. Results showed significant larger trunk flexion angle in flexion relaxation postures after the flexion relaxation protocol ( $\mathrm{p}$-value $<0.001)$ which demonstrated passive tissues in the trunk region had considerable creep deformation. What is more, reflex response was never happened after the flexion relaxation protocol ( $\mathrm{p}$-value $<0.029)$ and failed to recover to normal values in the tested recovery period. These changes will induce spinal instability and increase the spine injury risk. 
The change of active musculoskeletal system can also affect spinal stability. For instance, in one study a spinal stability biomechanical model was developed to evaluate the effect of dynamically induced muscle fatigue on spinal stability (Granata et al., 2004). Muscle fatigue was induced dynamically by lifting a $12.7 \mathrm{~kg}$ load repetitively from the ground level to an upright posture with the frequency of $60 \mathrm{lifts} / \mathrm{min}$ for at least 2 minutes. Results showed that the experimental protocol considerably raised the antagonist co-contraction level and reduced the spinal stability.

\subsubsection{Stability of the pelvis}

The role of the pelvis in the spinal stability is critical since pelvis is the foundation for the movement of the spinal column. As in the above example, the ball could never enter the stable status unless the surface that holds it is stabilized first. In the human daily movement, active

muscles (muscles in the trunk, hip and thigh, etc.) coordinate with the pelvic passive tissues to control the pelvic rotation and keep the spinal column stable. Previous studies have shown that the sacrotuberous ligaments and sacroiliac ligaments are of importance in pelvic stability (Wingerden et al., 1993; Vleeming et al., 1996). The sacrotuberous ligaments can stabilize the SI joint through the activation of the biceps femoris and gluteus maximus muscles and nutation of the sacrum while the sacroiliac ligaments can stabilize the SI joint during counter-nutation of the sacrum and activation of the erector spinae muscles and the tension of the ligament decreased during activation of the gluteus maximus and traction of lumbodorsal fascia (Figure 15). 


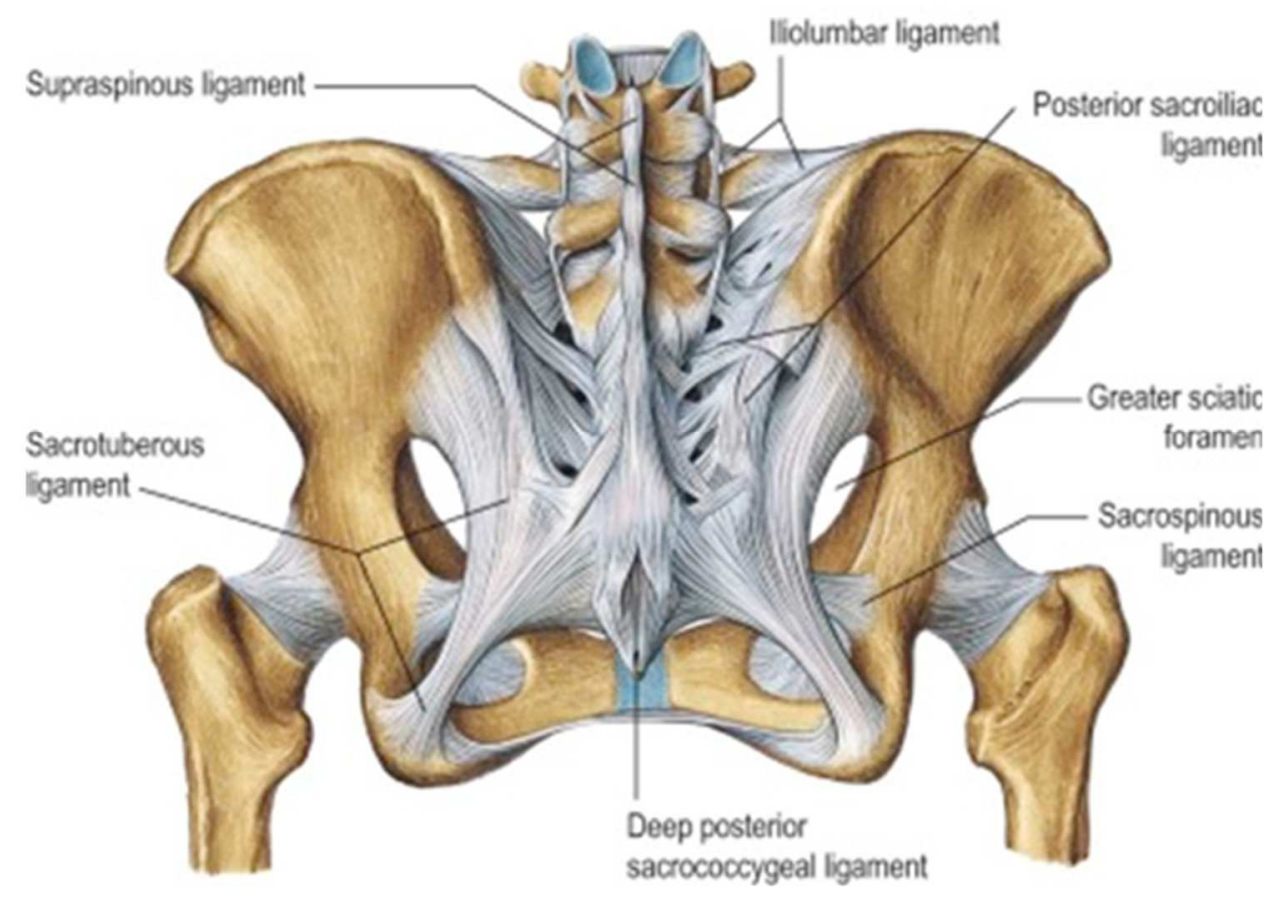

Figure 15: A demonstration of sacrotuberous ligaments and sacroiliac ligaments (from 2015 Gray's Anatomy)

\subsection{Problem statement}

LBP is a significant health problem with a high incidence rate and large economic cost. The exact etiology of LBP remains unclear. However, previous studies have identified a number of risk factors associated with LBP, such as high spinal compression and shear loading, muscle fatigue and lumbar tissue creep.

Uneven working surface is commonly found in a number of different occupational settings such as agriculture, fishing and transportation. Workplace health and safety organizations have reported that uneven surface could elevate the risk of developing LBP, but the underling mechanism remains unknown. FRP has been used as an important method to study the interaction between the active and passive lumber tissues in the lumbar region. Previous studies 
have made extensive efforts investigating lumbar FRP while study participants stand on flat, even ground surfaces. However, the effect of slanted ground surface on the lumbar FRP has not been well understood. Spine stability is critical to support body motion, undertake load and avoid injury. Knowledge regarding spinal stability on uneven surfaces could be used to prevent low back injuries.

Numerous ergonomic risk assessment tools and models have been developed in recent years. However, most of these risk assessment tools only consider common risk factors and do not take the working surface condition into consideration. Quantitative data are required to evaluate the influence of uneven surface on the risk of developing LBP before attempts to add a new "ground condition" parameter to existing ergonomic risk assessment models.

\subsection{Research questions}

The research questions of this research can be summarized as the following:

1) What are the effects of slanted ground surface on the spinal biomechanics and lumbar segmental stability?

2) Could the changes of spinal biomechanics and lumbar segmental stability due to the slanted ground surfaces be detectable from the current motion tracking devices and EMG systems?

3) Could the traditional COP based whole body stability analysis methods be applied to the local segmental stability analysis? 
4) Could the influences of slanted ground surfaces on spinal biomechanics and lumbar segmental stability be applicable to the current ergonomics assessment tools?

\subsection{Research method}

The current research study was designed to investigate the influence of uneven ground surfaces and asymmetric postures on the spinal biomechanical responses and lumbar segmental stability. A two-step approach was adopted. In experiment one, an experiment with a single independent variable was conducted to explore the effects of lateral slanted ground surfaces on spinal biomechanical response. FRP was selected to represent spinal biomechanical response. Based on the findings of experiment one, the second phase of the study was conducted to further explore the influences of antero-posteriorly slanted ground surface and asymmetric posture on spinal biomechanical responses and lumbar segmental stability. 


\section{CHAPTER 3. EXPERIMENT ONE}

\subsection{Overview}

As discussed in the previous section, in principle, the uneven ground condition has the potential to influence the lumbar biomechanical performance. However, it remains unclear whether the magnitude of the influence is detectable with the current equipment and the difference between conditions is statistically significant. Therefore, the purpose of the experiment one was to evaluate the effects of slanted ground surface on the lumbar biomechanical response. To be specific, FRP was selected to represent the lumbar biomechanical changes and was tested under different laterally slanted surfaces. Based on existing evidence, it was hypothesized that the change in the magnitude of laterally slanted surface would create discrepancy in bilateral lumbar muscle FRP during trunk flexion-extension motion.

\subsection{Participants}

Fourteen male participants were recruited from the student population at West Virginia University. Their average age, height and weight were 27.4 (SD 3.1) years old, 176.0 (SD 4.9) $\mathrm{cm}$ and 71.0 (SD 9.2) $\mathrm{kg}$, respectively. All participants were free from any chronic lower extremity or low back injuries. Participants with balance disorders were excluded from the study since the relative high risk of falling when standing on a slanted ground surface. Female participants were excluded from the current study due to the consideration of eliminating the potential confounding effects of gender. The experimental procedure was approved by the West Virginia University Institutional Review Board and written informed consent was obtained from all participants prior to data collection. 


\subsection{Apparatus}

Custom-made wood structures were built to simulate the laterally slanted ground surface $\left(15^{\circ}\right.$ and $30^{\circ}$ ) (Figure 16). Anti-slippery strips were attached to the surface to provide a higher friction coefficient to reduce the risk of falling. In addition, participants' stance width (distance between the center lines of their shoes) was controlled at their shoulder width across all conditions.

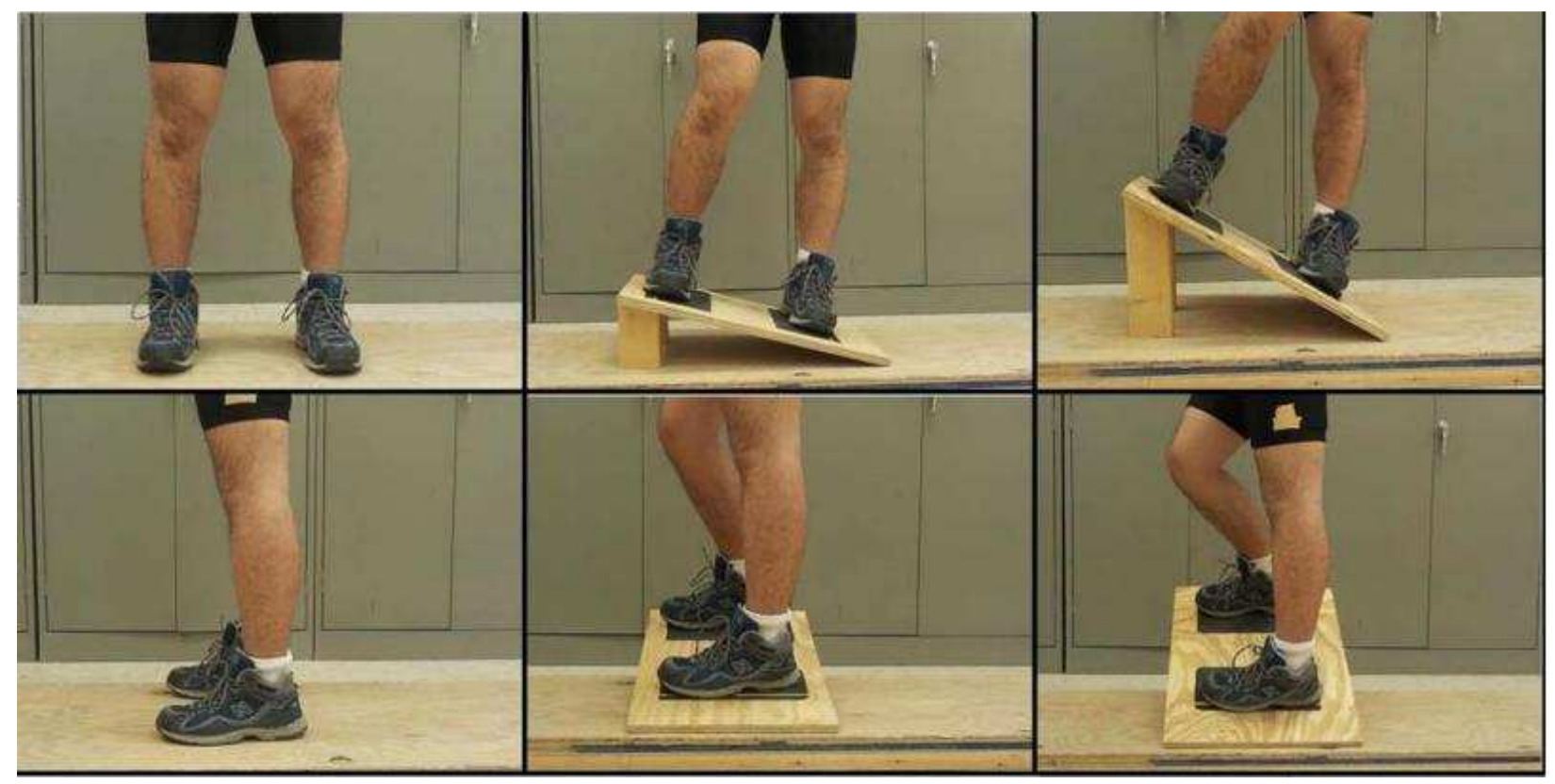

Figure 16: Side and frontal views of the slanted ground conditions: right panel, $30^{\circ}$ slanted; middle panel, $15^{\circ}$ slanted; left panel, flat ground

Muscular activity was collected from the erector spinae (lumbar paraspinal muscle at the L3 level) and multifidus (lumbar paraspinal muscle at the L4 level) using a surface electromyography (EMG) system (Bagnoli, Delsys, Boston, MA, USA) (Figure 17). Bipolar surface EMG electrodes were placed $2 \mathrm{~cm}$ lateral from the $\mathrm{L} 4$ spinous process and $4 \mathrm{~cm}$ lateral from the L3 spinous process (Ning et al., 2011) (Figure 18). The EMG signals were sampled at $1024 \mathrm{~Hz}$. 


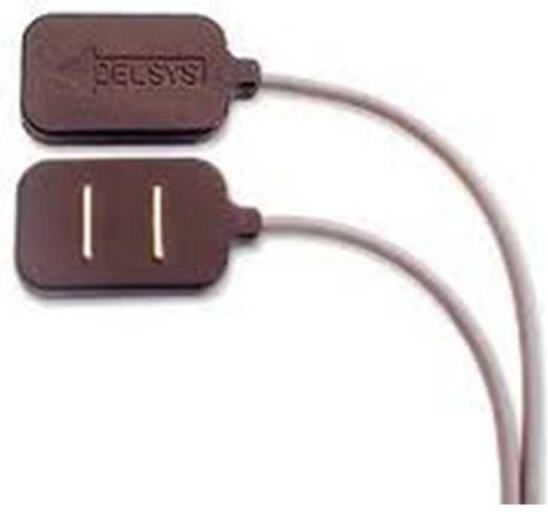

(a)

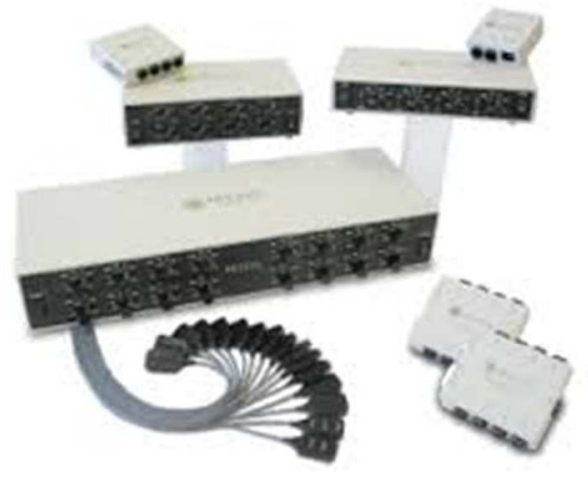

(b)

Figure 17: (a) Delsys biopolar EMG electrodes; (b) EMG signal amplifier

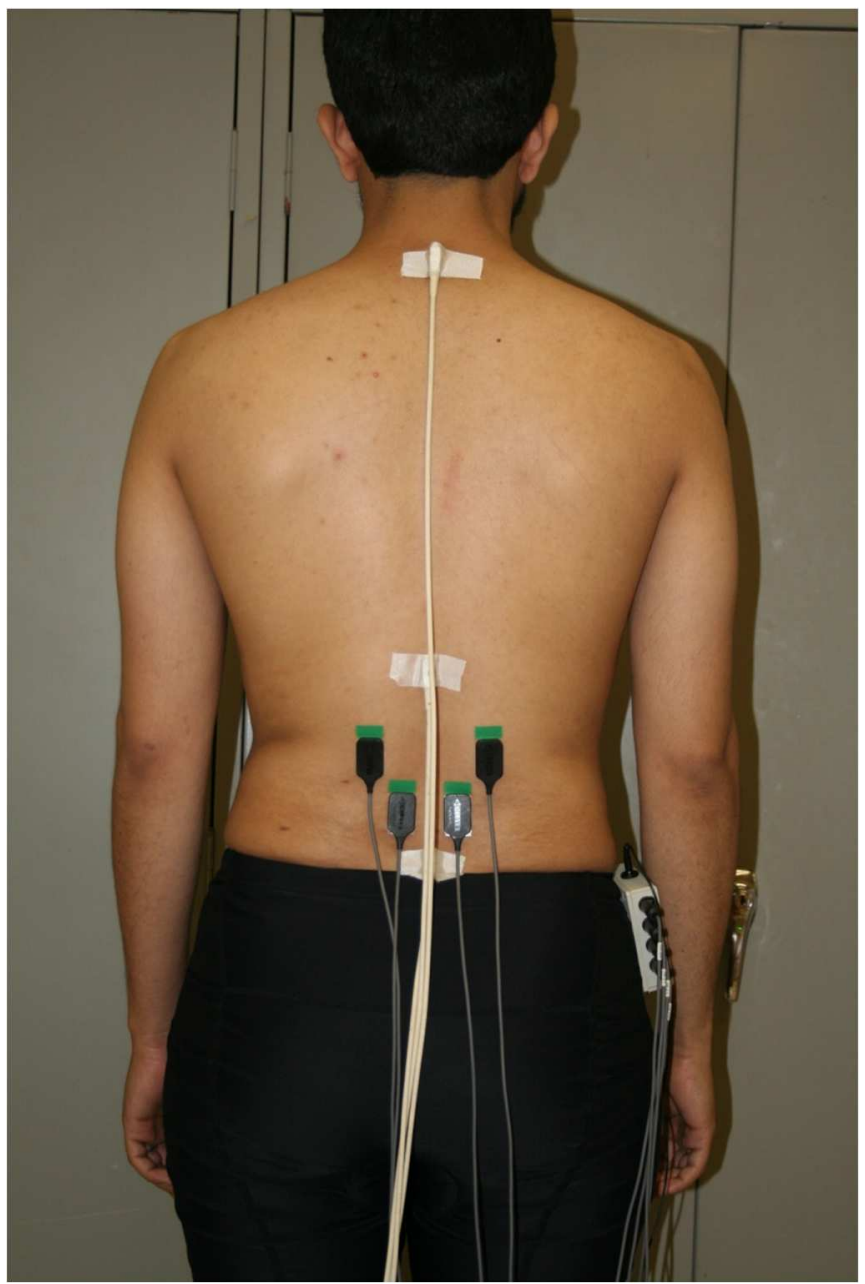

Figure 18: An illustration of the location of EMG sensors 
Participants' trunk kinematics data were collected by a magnetic field based motion tracking system (Motion Star, Ascension, Burlington, VT, USA) (Figure 19). Three motion sensors were attached to the skin surface over the C7, T12 and S1 vertebrae levels. During the data collection, the three dimensional coordinates and the sagittal, coronal and rotational angles of each motion sensor (regard to the magnetic field center) were sampled at $102.4 \mathrm{~Hz}$. The EMG and trunk kinematics data were synchronized and preprocessed using MotionMonitor software (Model MotionMonitor, Innovative Sports Training, Chicago, IL, USA). 


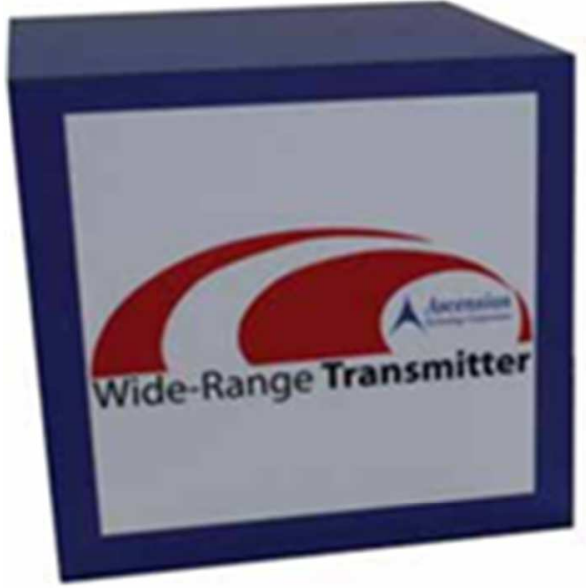

(a)

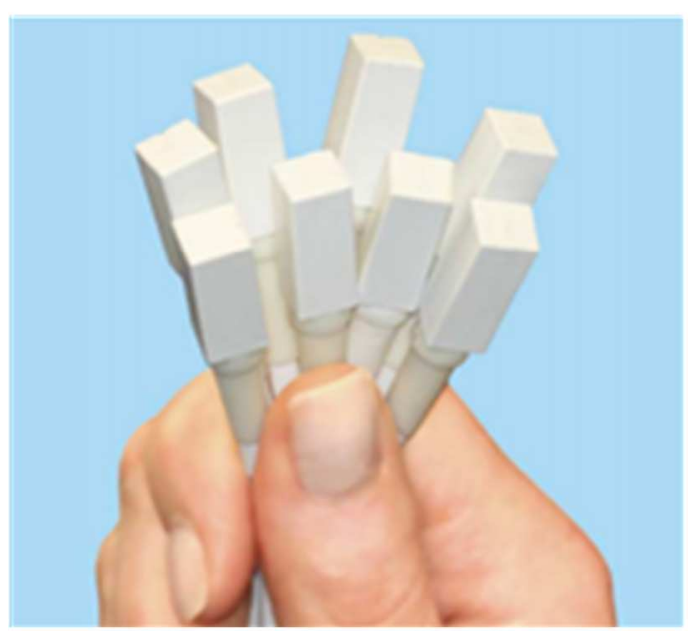

(c)

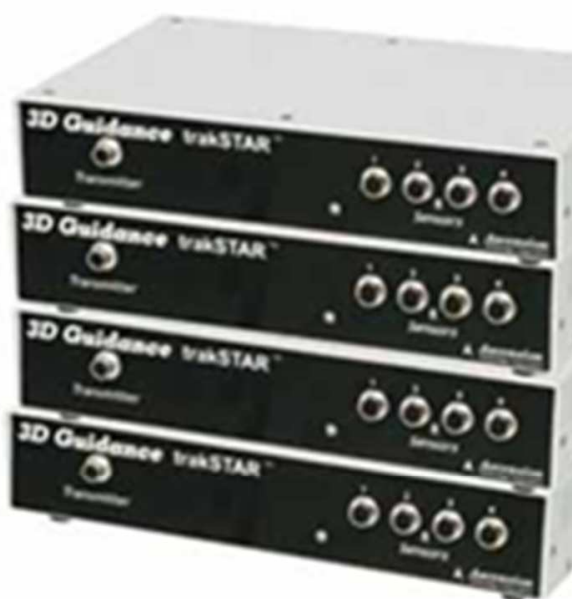

(b)

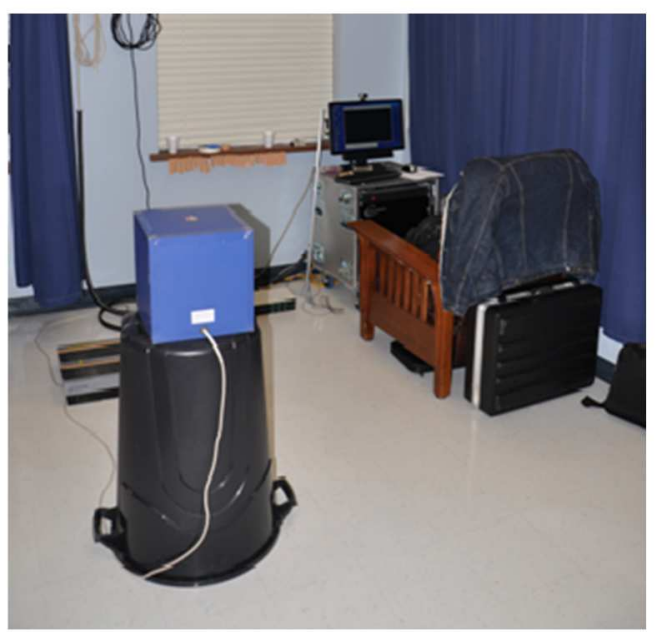

(d)

Figure 19: A demonstration of magnetic field based motion tracking system: (a) the transmitter which is the origin of the coordinate; (b) A/D board; (c) motion sensors; (d) an experiment setup

Participants' maximum voluntary contraction (MVC) data of the lumbar muscles were collected using a dynamometer (HUMAC Norm, Computer Medicine, Stoughton, MA, USA) (Figure 20). 


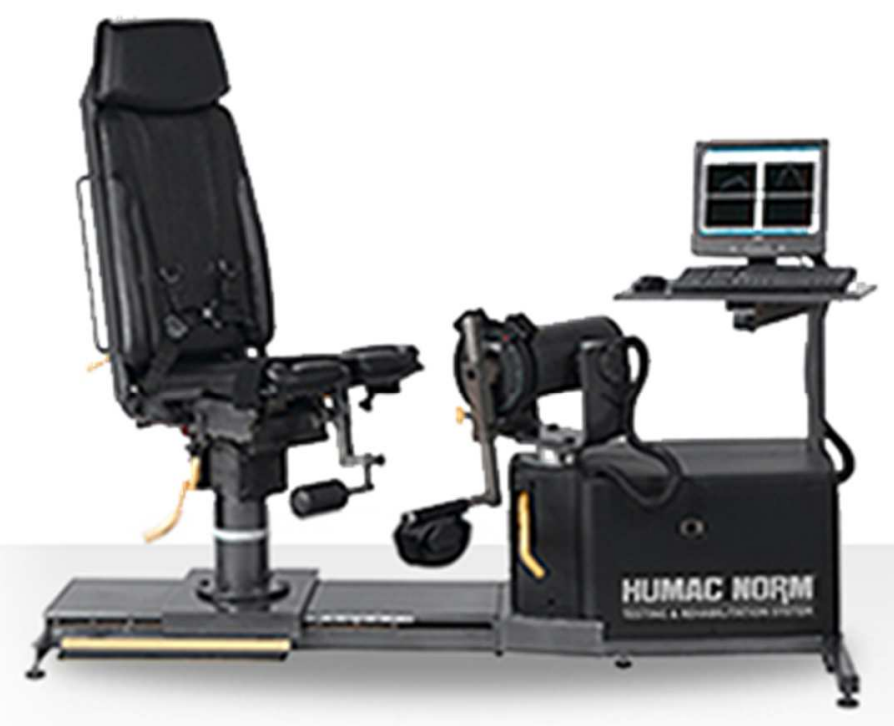

Figure 20: The dynamometer used in the current study

\subsection{Experimental design}

\subsubsection{Independent variables}

The ground surface angle (ANGLE) was the only independent variable involved in this experiment and it was evaluated at three levels: $0^{\circ}$ (flat ground), $15^{\circ}$ and $30^{\circ}$ (laterally slanted ground). The slanted angle in this experiment was defined as the angle between the ground surface and the horizontal plane. Based on results from a pilot test, the direction of the laterally slanted surface (whether the right side is higher or the left side is higher) does not affect the lumbar biomechanical response. Therefore, during the data collection participants were required to always have their right foot placed at the higher position on the slanted surface to simplify the experimental procedure (Figure 16). In the current experiment, muscles on the right side would 
be referred as the contralateral side while muscles on the left side would be referred as the ipsilateral side.

\subsubsection{Dependent variables}

Ten dependent variables were evaluated in this experiment and they can be divided into two categories: lumbar flexion angle and trunk inclination angle. Lumbar flexion angle was calculated as the angular difference between the pitch angles (in the sagittal plane) of the T12 and S1 motion sensors while trunk inclination angle was defined as the angle between the normal line and the line connects the C7 and S1 motion sensors (Figure 18). A natural upright posture will generate a close to zero trunk inclination angle and a negative lumbar flexion angle (represents the lordosis of the lumbar spine) (usually around $-20^{\circ}$ ). The first two dependent variables were the maximum lumbar flexion angle and the maximum trunk inclination angle which were defined as the peak angles at the full flexion posture. The other eight dependent variables were defined as the corresponding lumbar flexion and trunk inclination angles at the onset point of FRP during trunk flexion motion for the four sampled low back muscles: left erector spinae (ipsilateral L3 paraspinals), right erector spinae (contralateral L3 paraspinals), left multifidus (ipsilateral L4 paraspinals) and right multifidus (contralateral L4 paraspinals). The

onset of FRP is referred to as the "EMG-off" point to better reflect the cessation of muscular EMG activity at the beginning of FRP. 


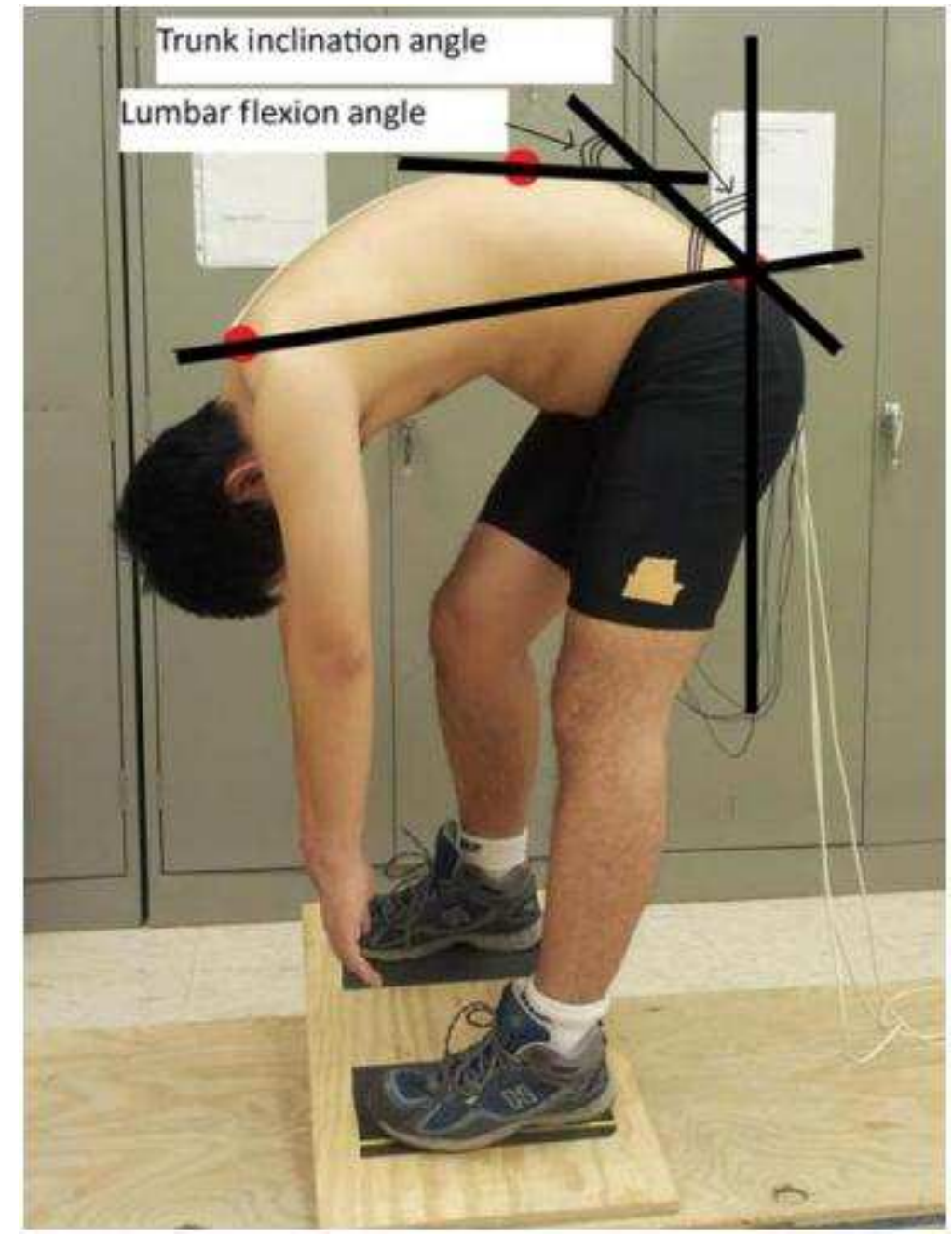

Figure 21: Illustration of the definition of lumbar flexion angle and trunk inclination angle

\subsection{Protocol}

Upon arrival at the work site, the experimental procedure was described to the participant followed by a warm up session ( 5 minutes). The purpose of the warm up session was letting the participant get familiar with the equipment and also prevent muscle strain. Surface electrodes and motion sensors were then attached to the designed locations with double-sided tape according to instructions from previous literature (Ning et al., 2011). During the data collection phase, each participant performed a total of 15 trunk flexion-extension tasks ( 3 ANGLE x 5 
repetitions) in a random order. The order of the trials has been randomized to eliminate the influence of the learning effect. The experimental protocol required the participant to perform a slow and smooth trunk flexion-extension motion: 7 seconds to flex the trunk from upright standing to the full flexion posture, remained in full flexion posture for 6 seconds and 7 seconds to come back to the upright standing position. The full flexion posture was defined as the maximum trunk flexion that one can maintain with all trunk muscles relaxed. A metronome was used to assist the participant to better control the pace of the motion. At least 1 minute of rest was provided between trials in order to avoid the effect of lumbar muscle fatigue and viscoelastic changes of posterior lumbar tissues.

\subsection{Data processing}

The unprocessed raw EMG data were first filtered with a low pass frequency of $500 \mathrm{~Hz}$, a high pass frequency of $10 \mathrm{~Hz}$ and a notch filter of $60 \mathrm{~Hz}$ and its aliases. The data were then fully wave rectified. A moving window filter (half second width) was applied to generate a standard deviation profile for each muscle. The standard deviation at the full flexion posture was selected as the full flexion standard deviation (FSD). The standard deviation profile from each muscle was then compared with its corresponding FSD in order to identify the onset and cessation of FRP. The last point where the standard deviation of a particular muscle is larger than $3 *$ FSD during trunk flexion motion was defined as the EMG-Off point and the corresponding lumbar flexion and trunk inclination angles were defined as the lumbar/trunk EMG-Off angles for each muscle accordingly. 


\subsection{Statistical analysis}

A general linear model was used to perform the statistical analysis:

$$
Y_{i j}=\mu+\tau_{i}+\beta_{j}+\varepsilon_{i j k}
$$

Where $\mathrm{Y}_{\mathrm{ij}}, \mu, \tau_{\mathrm{i}}, \beta_{\mathrm{j}}$, and $\varepsilon_{\mathrm{ijk}}$ represent biomechanical responses (dependent variables), overall mean, main effect of independent variable, block effect and random error. Total sum of squares was calculated as:

$$
S S_{T}=\sum_{i=1}^{a} \sum_{j=1}^{b} \sum_{k=1}^{n} Y_{i j k}^{2}-\frac{y_{\ldots}^{2}}{a b n}
$$

Sums of squares for the main effects and block effects were found as follows:

$$
\begin{aligned}
& S S A=\frac{1}{b n} \sum_{i=1}^{a} y_{i . .}^{2}-\frac{y_{\ldots}^{2}}{a b n} \\
& S S B=\frac{1}{a n} \sum_{j=1}^{b} y_{. j .}^{2}-\frac{y_{\ldots}^{2}}{a b n}
\end{aligned}
$$

In the statistical analysis phase, each repetition was treated as a single observation. Prior to any statistical analysis, the assumptions of the analysis of variance (ANOVA) procedures (i.e. independence of observations, normal distribution of residuals and constant variance of residuals) were tested. Variables that did not satisfy one or more assumptions were transformed to meet the criteria (Montgomery 2012). The effects of ANGLE on each dependent variable were then evaluated with repeated measures ANOVA with 'participant' considered as the random factor. 
To further identify the differences between the three ANGLE conditions, Tukey-Kramer posthoc test was used on the dependent variables that were significantly affected by ANGLE. Finally, paired t-tests were performed to investigate the differences between the EMG-off angle of the bilateral low back muscles. The criteria p-value was 0.05 for all statistical analysis.

\subsection{Results}

First of all, the EMG data demonstrated that the FRP consistently occurred in all four back muscles in all conditions. Results indicated that the maximum lumbar flexion angle was not significantly affected by ANGLE while the maximum trunk inclination angle was considerably lower in the $30^{\circ}$ ground angle condition (on average $2.8^{\circ}$ lower than flat ground condition) (Figure 22 and Figure 23). In all figures bars indicate corresponding standard error. This result was probably caused by the fact that participants' bended right knee would physically limit the range of trunk motion. In addition, the increase in ANGLE reduced the lumbar EMG-Off angle on the left (ipsilateral) side of both the erector spinae and multifidus (Figure 24 and Figure 25), whereas the right (contralateral) side was not affected. Regarding the trunk EMG-Off angle, the ipsilateral side of both the erector spinae and multifidus demonstrated significantly lower values with the increase in ANGLE (Figure 26 and Figure 27). However, different from the results of the lumbar EMG-Off angles, the contralateral sides of both muscles indicated significantly lower trunk EMG-Off angles in the slanted ground conditions. This difference could be attributed to the lower maximum trunk inclination angle at these conditions.

Furthermore, results of the paired t-test discovered considerable different lumbar and trunk EMG-Off angles between the ipsilateral and contralateral sides of both the erector spinae and 
multifidus under both slanted ground conditions (Table 5). Our findings suggest that when performing deep trunk flexion motion on laterally slanted ground surfaces, the ipsilateral lumbar muscles will always cease activity earlier than the contralateral lumbar muscles. Results in Table 5 suggest the angular differences between the contralateral and ipsilateral lumbar muscle EMGoff angles. As shown in the table, these angular differences were more significant among the erector spinae than the multifidus. For instance, at $15^{\circ}$ slanted ground condition, $3.7^{\circ}$ versus $1.8^{\circ}$ trunk EMG-Off angle difference between the bilateral erector spinae and multifidus, respectively.

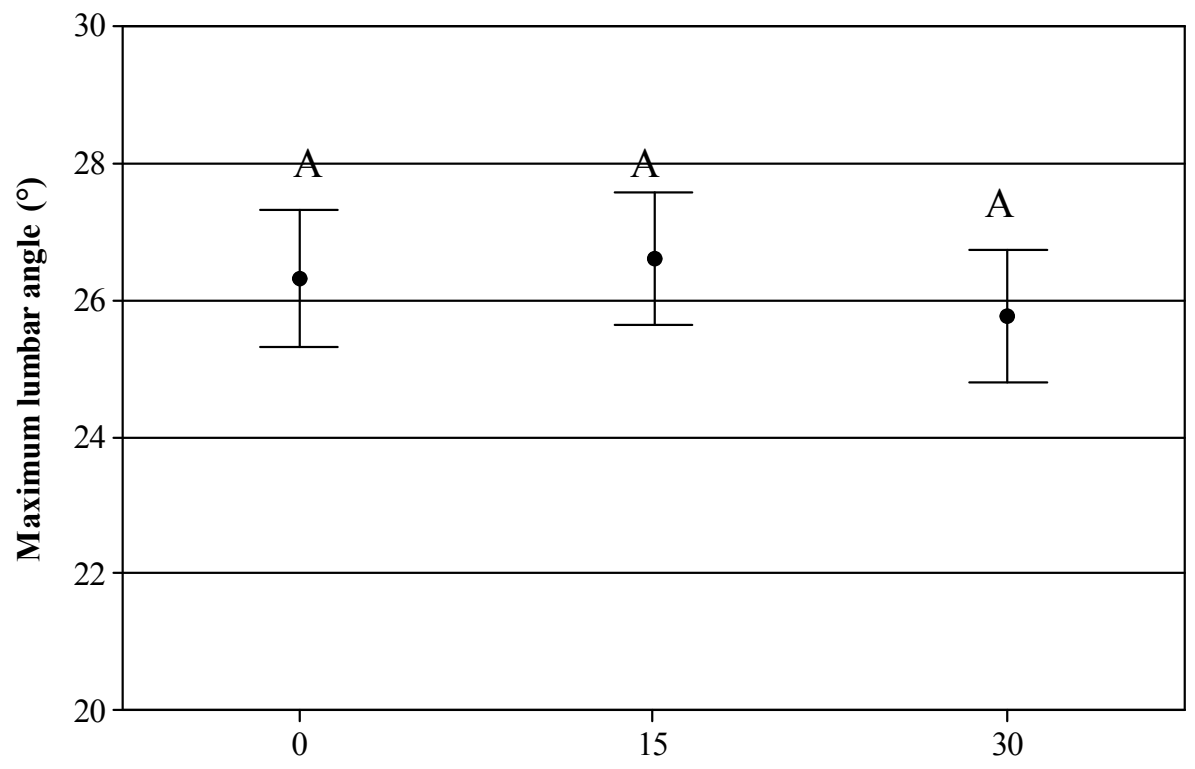

Figure 22: Maximum lumbar flexion angle under different ground conditions. Different letters denote angles that are statistically different from one another 


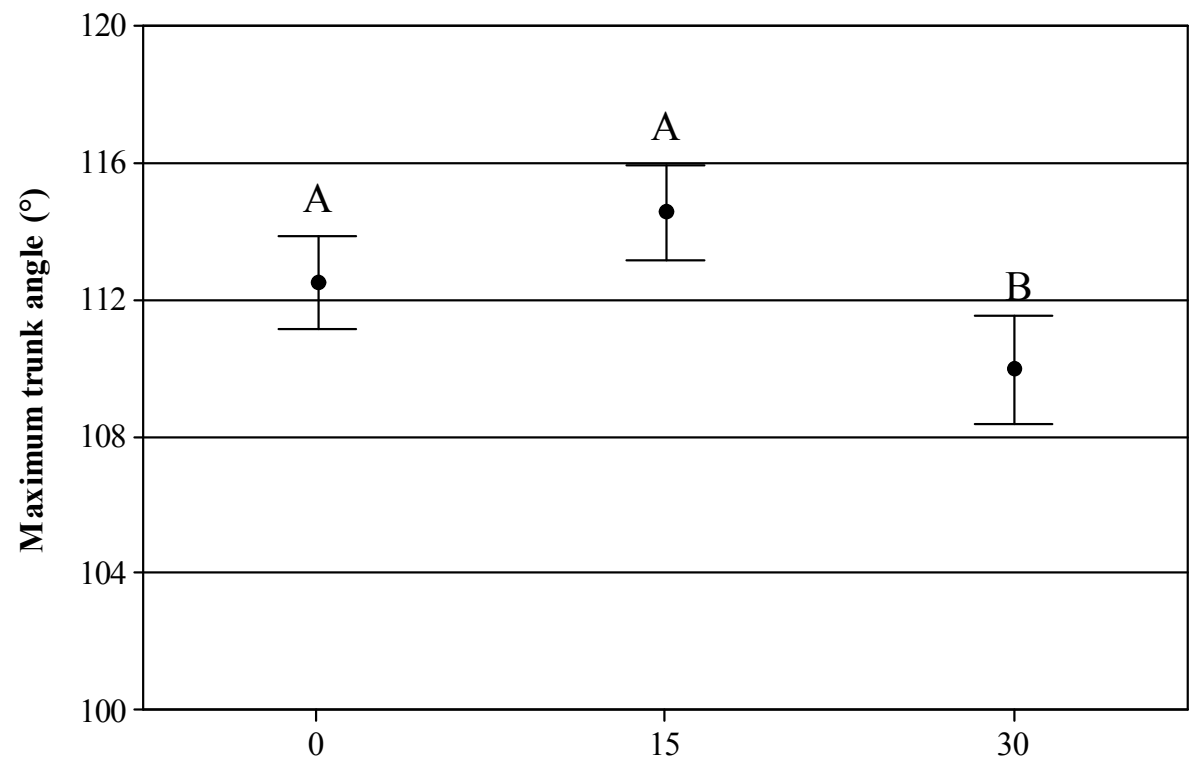

Figure 23: Maximum trunk inclination angle under different ground conditions. Different letters denote angles that are statistically different from one another

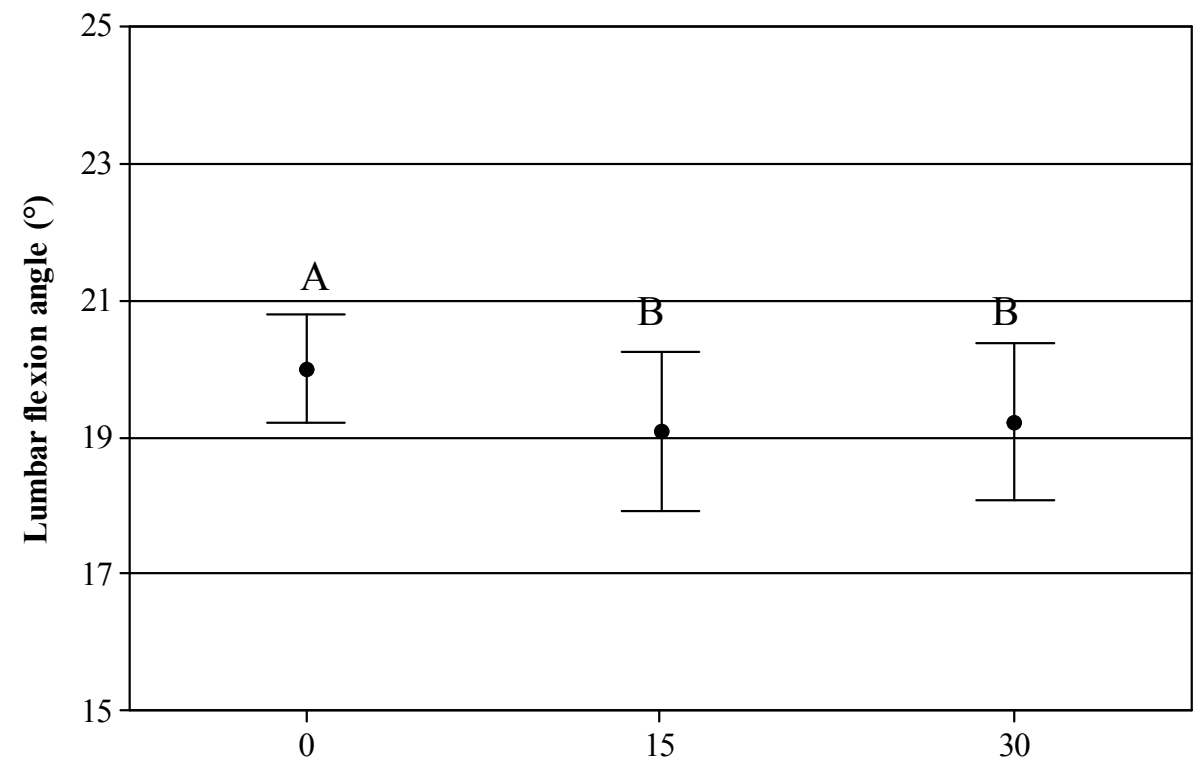

Figure 24: Lumbar EMG-Off angle for the ipsilateral side of erector spinae under different ground conditions. Different letters denote angles that are statistically different from one another 


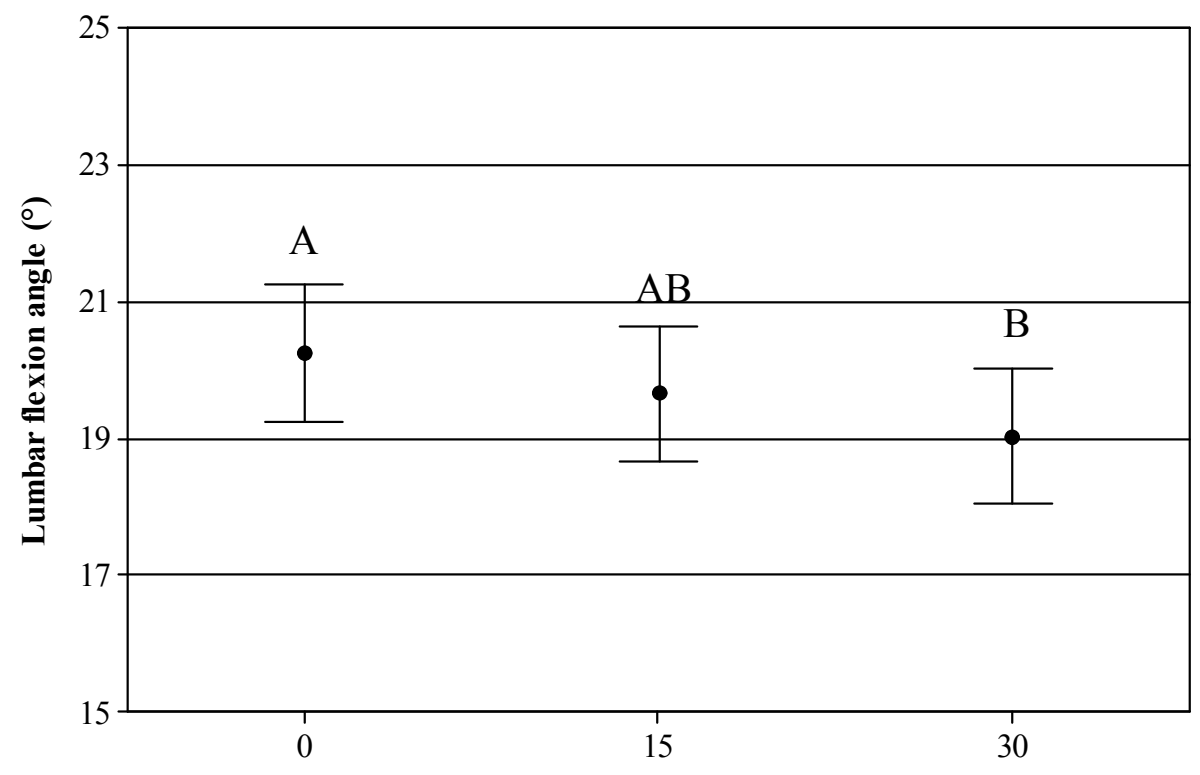

Figure 25: Lumbar EMG-Off angle for the ipsilateral side of multifidus under different ground conditions. Different letters denote angles that are statistically different from one another

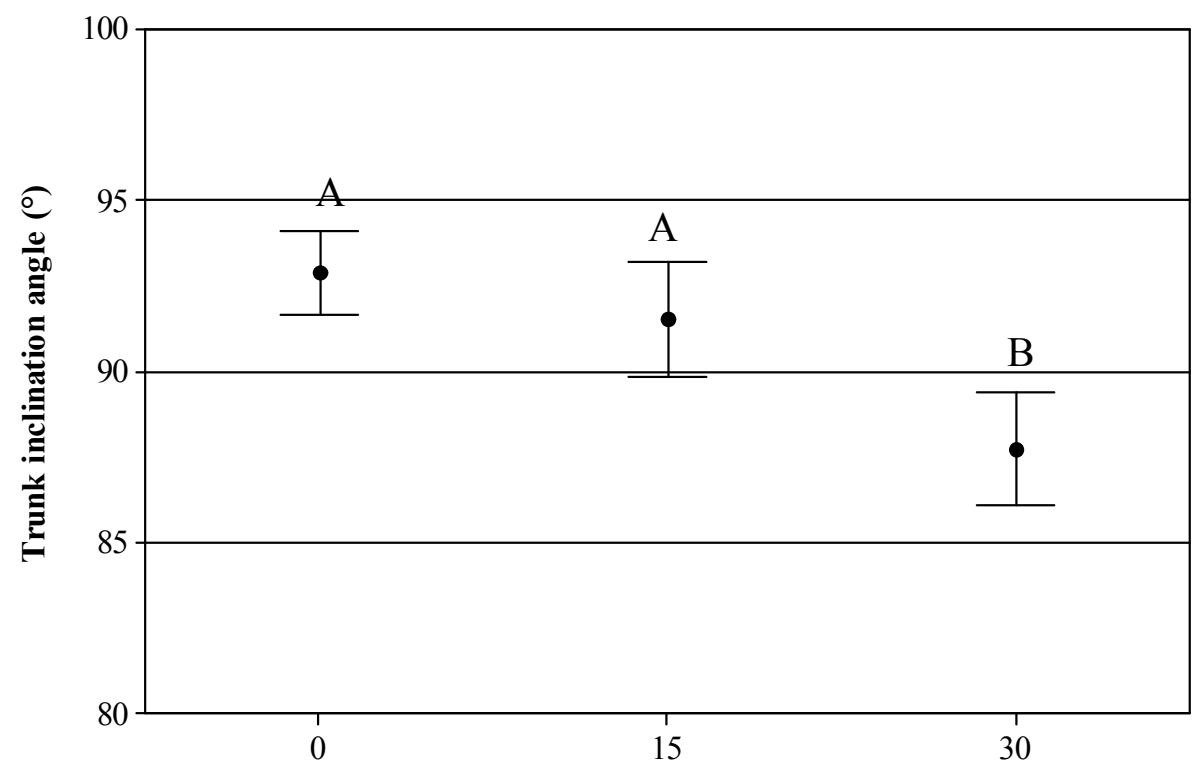

Figure 26: Trunk EMG-Off angle for the ipsilateral side of erector spinae under different ground conditions. Different letters denote angles that are statistically different from one another 


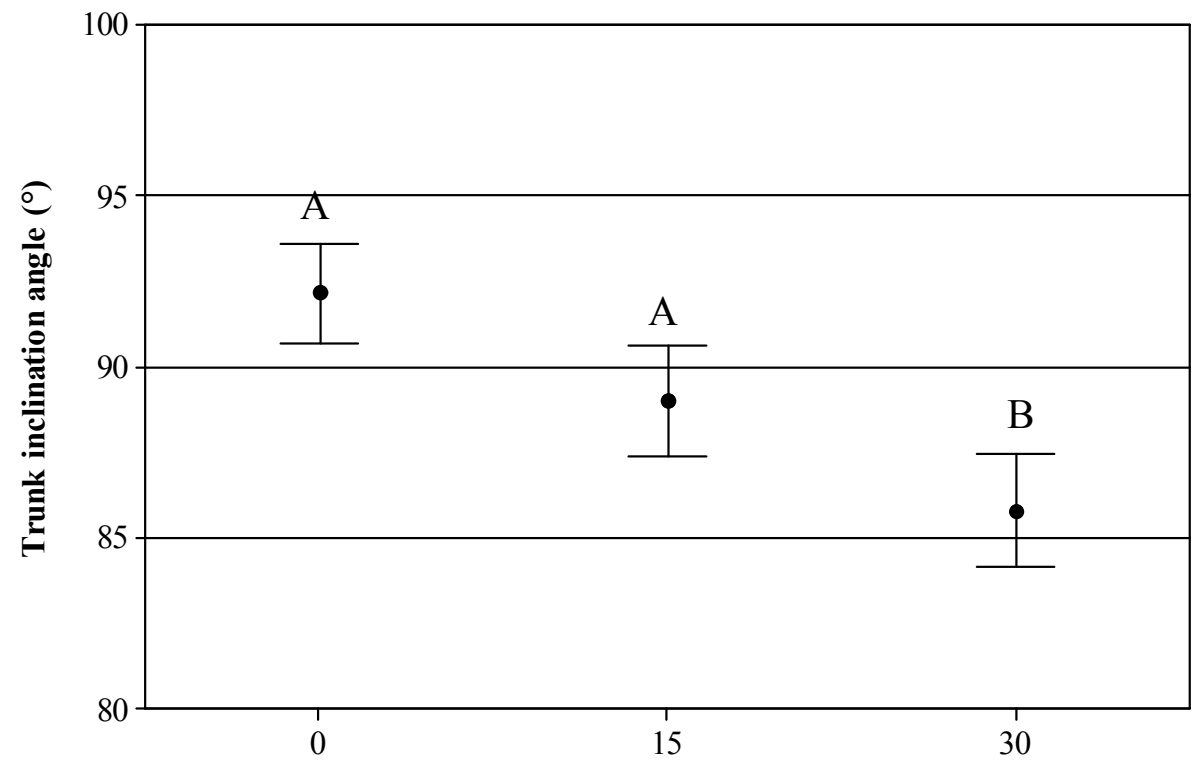

Figure 27: Trunk EMG-Off angle for the ipsilateral side of multifidus under different ground conditions. Different letters denote angles that are statistically different from one another

Table 5: The EMG-Off angle difference (right minus left) between erector spinae and multifidus

\begin{tabular}{|c|c|c|c|c|}
\hline \multirow[b]{2}{*}{ Angle } & \multicolumn{4}{|c|}{ Erector Spinae (difference) } \\
\hline & \multicolumn{2}{|c|}{ Lumbar EMG-off } & \multicolumn{2}{|c|}{ Trunk EMG-off } \\
\hline 0 & -0.1 & $\mathrm{p}=0.68$ & 0.1 & $\mathrm{p}=0.91$ \\
\hline 15 & 1.9 & $\mathbf{p}<\mathbf{0 . 0 0 1}$ & 3.7 & $\mathrm{p}=\mathbf{0 . 0 1 7}$ \\
\hline \multirow[t]{2}{*}{30} & 2 & $\mathbf{p}<0.001$ & 3.9 & $\mathbf{p}<0.001$ \\
\hline & \multicolumn{4}{|c|}{ Multifidus (difference) } \\
\hline Angle & \multicolumn{2}{|c|}{ Lumbar EMG-off } & \multicolumn{2}{|c|}{ Trunk EMG-off } \\
\hline 0 & 0 & $\mathrm{p}=0.72$ & -0.2 & $\mathrm{p}=0.77$ \\
\hline 15 & 1.1 & $\mathbf{p}<0.001$ & 1.8 & $p=0.043$ \\
\hline 30 & 1.7 & $p<0.001$ & 2.8 & $p=0.001$ \\
\hline
\end{tabular}




\subsection{Discussion}

Results showed that ipsilateral side of the erector spinae and multifidus ceased activity earlier under the slanted ground conditions. This result confirms our hypothesis that the uneven ground condition could affect spinal biomechanical response. Two potential mechanisms could explain this change. First of all, we observed a small but significant rightward shift of the $\mathrm{C} 7$ motion sensor during the trunk flexion motion under the two slanted ground conditions (Figure 28). This displacement in the mediolateral direction showed a shift of trunk motion toward the right side and induced a slight asymmetric bending status. Although the experiment was designed to be sagittally symmetric, when performing the trunk flexion task on laterally slanted surfaces, participants may have used a slight asymmetric trunk posture as a strategy to maintain their balance. This slight change in posture, although barely notable, could influence the back muscle activity patterns significantly. Previous study has shown that asymmetric trunk flexion introduces greater tension to the lumbar posterior tissues on the opposite side of asymmetry and this tension leads to an earlier onset of muscle relaxation. Results of the current pilot study supported this finding. Another alternative explanation came from the knee flexion point of view. Compared to the flat ground condition where both legs were kept straight, under the laterally slanted ground conditions the straight (left) could experience higher stress and strain due to the flexed knee of the other leg (right). This increase in strain would increase the tension on the hamstring muscles (e.g. biceps femoris) which could reduce forward pelvic rotation and increase the tension on lumbar posterior ligaments and the passive tension on the lumbar extensor muscles (Shin et al., 2004). This increased lumbar ligament tension could cause an early cessation of activity for lumbar muscles on the ipsilateral side. 
The FRP of the erector spinae and multifidus on the contralateral side was also affected by the slanted ground surfaces. As noted, participants kept a flexed right knee posture while standing on laterally slanted ground surfaces. The flexion of knee is known to cause a forward pelvic rotation (Murray et al., 2002) which generate reduced tension on passive lumbar tissues and should therefore delay the onset of FRP for the lumbar muscles on the corresponding side. To compensate for the reduction of the passive tissue loading, the lumbar muscles need to exert more force for a longer duration during the trunk flexion-extension motion. Results of the pilot study supported this assertion. The right erector spinae and multifidus lumbar EMG-off angles demonstrated a small but steady increase from $19.9^{\circ}$ to $21.1^{\circ}$ and $20.3^{\circ}$ to $20.8^{\circ}$, respectively, with an increase in slanted ground angle. Nevertheless, this result was not statistically significant, possibly due to the relatively small knee flexion angles adopted by the participants.

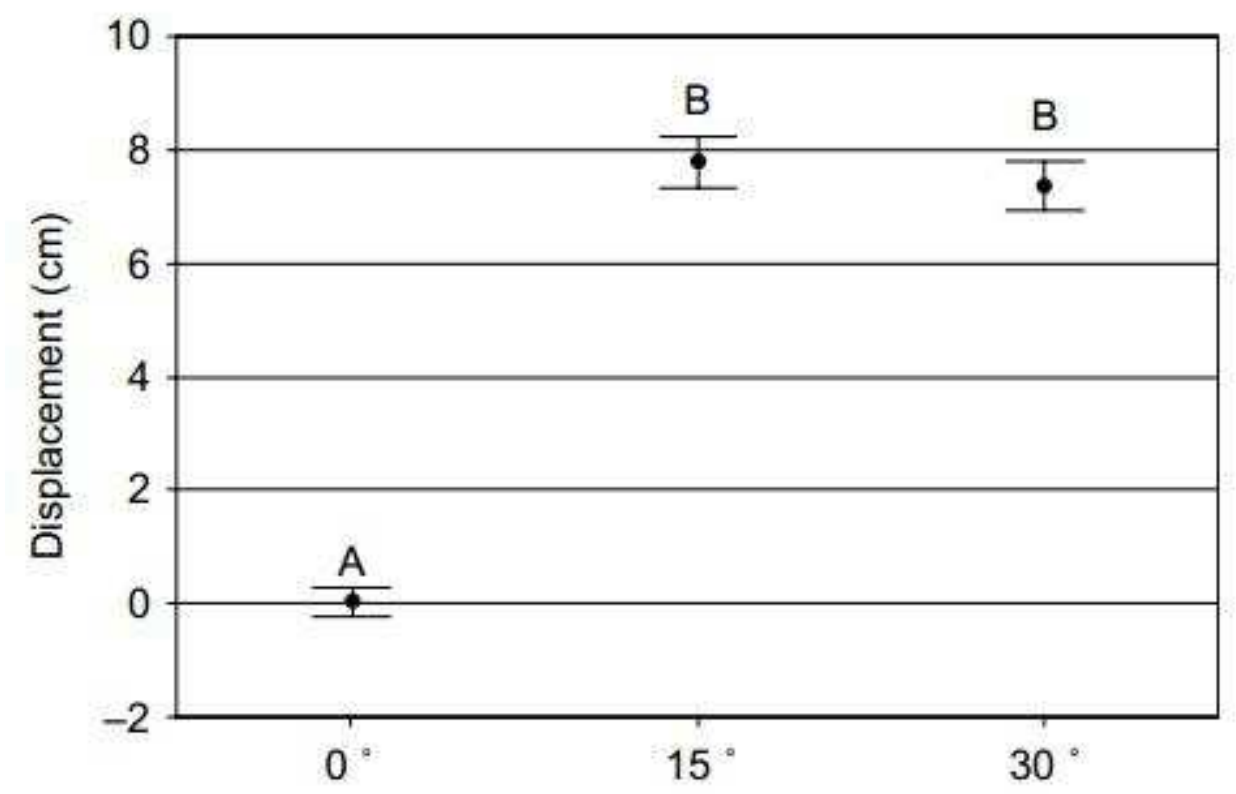

Figure 28: Mediolateral displacement of $\mathrm{C} 7$ sensor during trunk flexion motion. Positive value indicates rightward shift. Different letters denote displacements that are statistically different from one another 
In addition, the comparison between the bilateral erector spinae and multifidus revealed that the ipsilateral lumbar muscles cease activity earlier than the contralateral lumbar muscles. The difference is more pronounced among the erector spinae than the multifidus. A previous study reported that the laterally located back muscles are more affected by asymmetrical motion during trunk bending (Ning et al., 2011). In the current study, the EMG sensors on the erector spinae were more laterally placed than the multifidus $(4 \mathrm{~cm}$ vs. $2 \mathrm{~cm}$ away from the mid-line of spinal column). This coupled with the slight rightward bending adopted by the participants during the trunk flexion motion may have produced the EMG-off angle difference between the bilateral erector spinae and multifidus. Furthermore, according to lumbar spine anatomy and the sampling locations of the erector spinae and multifidus muscles, it was suspected that the recorded EMG signals from the erector spinae may include some components came from more laterally located lumbar extensor muscles such as the longissimus and iliocostalis and signals from the mulifidus may primarily came from more medially located muscles. The longissimus is considered a global muscle with a primary function of providing the necessary force and moment during trunk motion while the multifidus is considered as a local lumbar muscle with a primary function of maintaining lumbar stiffness and stability (Bergmark 1989; Ward et al., 2009). It is suspected that the local muscles are less responsive to the global trunk motion in response to slanted ground surfaces.

\subsection{Summary}

Results of experiment one verified that slanted ground condition could significantly influence the lumbar biomechanical response during dynamic motion. This finding provides support and foundation to the second phase of study which would continue to evaluate the effects the uneven 
ground surface on spinal biomechanical response. The main findings from the pilot study have been published on Ergonomics (Hu et al., 2013).

Meanwhile, there are several aspects of the experimental design can be further improved. First of all, the current design involved knee flexion and the magnitude of knee flexion was not balanced bilaterally. Previous literature showed that different knee flexion can significantly affect spinal biomechanical responses. To prevent the potential confounding effect and mainly investigate the influence of slanted surface, knee flexion would be restricted in the second phase of study.

In addition, the uneven surface will be changed into the antero-posterior direction. This would also help in eliminating the unbalanced knee flexion. Another major advantage of anteroposterior direction slanted surface is that it could eliminate the asymmetric tendency during the trunk flexion task. In the pilot study, participants demonstrated unconscious asymmetric bending when standing on laterally slanted surface. Since it has been reported that asymmetry had strong effect on spinal biomechanical responses, the second phase of the study will include asymmetry as an extra independent variable and the interaction of slanted surface and asymmetry would also be explored. 


\section{CHAPTER 4. EXPERIMENT TWO}

\subsection{Overview}

The objective of the experiment two was to explore the effects of antero-posteriorly slanted ground surfaces and trunk asymmetry on spine biomechanics during trunk bending. More specifically, we investigated the influences of antero-posteriorly slanted ground surfaces, trunk asymmetry and their interaction on the lumbar extensor muscle FRP during trunk flexion motion as well as lumbar segmental stability performance during deep trunk bending posture. Existing evidence suggested that prolonged standing on antero-posteriorly slanted surfaces could generate posture adaptions (Gallagher et al., 2012). Moreover, tension on the hamstring muscles could influence the lumbar biomechanics and the load-sharing mechanism between active and passive lumbar tissues (Dewberry et al., 2003; Shin et al., 2004). Therefore, it was hypothesized that standing on an uphill facing antero-posteriorly slanted surface would reduce the onset angles of lumbar muscle FRP while standing on a downhill facing antero-posteriorly slanted surface will increase the onset angles of lumbar muscle FRP during trunk bending. Based on the results of a previous study (Ning et al., 2011), an earlier onset of FRP on the contralateral side of lumbar extensor muscles was also expected during asymmetric bending. In addition, previous literature has reported sloped surfaces significantly increased participants' standing postural instability (Simeonov et al., 2003; Lin and Nussbaum 2012). As a result, we expected to observe reduced lumbar segmental stability with a deep flexion posture when standing on antero-posteriorly slanted surface. 


\subsection{Participants}

The power analysis based on our pilot data suggested a sample size of twelve participants (with $\alpha$ $=0.05$ and 0.8 power). In the current study, a total of fourteen male volunteers were recruited. Their average age, body height and weight were 26.5 (SD 2.6) years, 174.3 (SD 8.8) cm and 67.0 (SD 9.5) kg, respectively. Participants with a history of back surgery in the previous 12 months or currently experiencing discomfort in their lower extremities were excluded from the study.

\subsection{Data collection instrumentation}

A surface EMG system (Bagnoli, Delsys, Boston, MA, USA) was used to sample muscular activities from the lumbar extensor muscles. All signals were amplified with a factor of 1000 and the sampling frequency was set at $1024 \mathrm{~Hz}$. Four EMG electrodes were placed bilaterally at erector spinae (4 $\mathrm{cm}$ laterally from the $\mathrm{L} 3$ spinous process) and multifidus ( $2 \mathrm{~cm}$ laterally from the L4 spinous process). To collect trunk kinematics data, an electromagnetic field based motion tracking system (Motion Star, Ascension, Burlington, VT, USA) that consists of three magnetic motion sensors was used in the current study. Motion sensors were secured to the skin over the C7, T12 and S1 vertebrae. Custom-made wooden structures were used to generate the slanted ground surfaces. Anti-slip strips were attached to the wooden surface in order to increase the coefficient of friction and reduce the risk of falling. 


\subsection{Experimental design}

\subsubsection{Independent variables}

The current experiment evaluated two independent variables: 1) Slanted ground surface (GROUND): two different antero-posteriorly slanted surfaces (-15 , i.e. uphill facing and $15^{\circ}$, i.e. downhill facing) and one flat ground $\left(0^{\circ}\right)$ surface were tested in the current study (Figure 29); 2) Trunk asymmetry (ASYM): while standing on different ground surfaces, participants were required to perform trunk bending in $0^{\circ}$ (sagittally symmetric) and $30^{\circ}$ asymmetric directions. Pilot study had confirmed that asymmetric direction (i.e. whether rotates toward the left or right) does not affect the lumbar biomechanical response, therefore, in the study, all asymmetric trials were conducted toward leftward from the mid-sagittal plane.

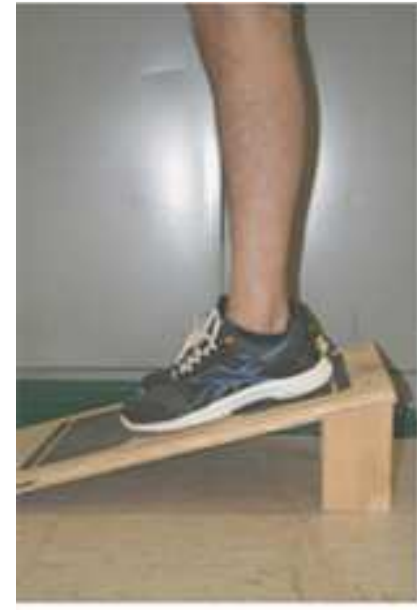

(a)

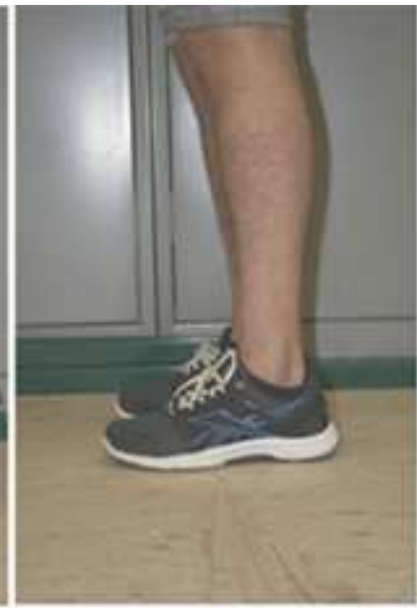

(b)

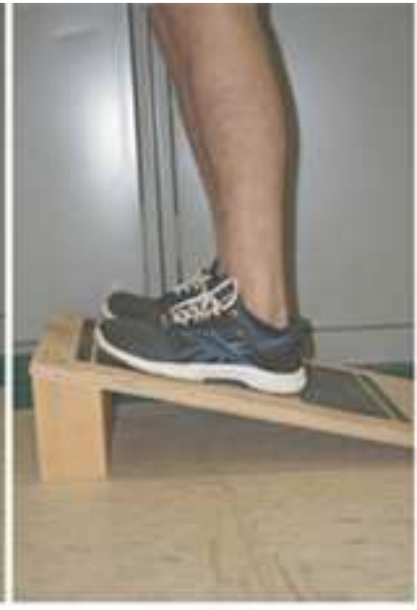

(c)

Figure 29: (a) side view of the $15^{\circ}$ downhill facing condition; (b) side view of the flat ground condition; (c) side view of the $-15^{\circ}$ uphill facing condition 


\subsubsection{Dependent variables}

\subsubsection{FRP related variables}

There were a total of 10 dependent variables in this category: 1) maximum lumbar flexion angle (MLF); 2) maximum trunk inclination angle (MTI); 3) left erector spinae (L3 paraspinal) EMGoff lumbar angle (LL3L); 4) left erector spinae (L3 paraspinal) EMG-off trunk angle (LL3T); 5) right erector spinae (L3 paraspinal) EMG-off lumbar angle (RL3L); 6) right erector spinae (L3 paraspinal) EMG-off trunk angle (RL3T); 7) left multifidus (L4 paraspinal) EMG-off lumbar angle (LL4L); 8) left multifidus (L4 paraspinal) EMG-off trunk angle (LL4T); 9) right multifidus (L4 paraspinal) EMG-off lumbar angle (RL4L) and 10) right multifidus (L4 paraspinal) EMGoff trunk angle (RL4T). Definition of lumbar flexion angle and trunk inclination remains the same as in the pilot work.

\subsubsection{Lumbar segmental stability related variables}

The stability analysis methods were selected from previous literature and those methods werecommonly used in traditional COP based stability analysis (Prieto et al., 1996, Lin \& Nussbaum, 2012). The sway motion of each segment was jointly defined by antero-posterior (AP) and medio-lateral (ML) coordinates (Figure 30). In the following equations, all summations are from 1 to $\mathrm{N}$, unless indicated otherwise while $\mathrm{N}$ is the total number of data points in a particular trial. The $\mathrm{AP}_{\mathrm{O}}$ and $\mathrm{ML}_{\mathrm{O}}$ time series define the sway path relative to the origin of the motion tracking system (Equation 4-1 and 4-2):

$$
\overline{A P}=1 /{ }_{N} \sum A P_{O}[n]
$$




$$
\overline{M L}=1 /{ }_{N} \sum M L_{O}[n]
$$

To simplify the following definitions, the AP and ML time series are referenced to the mean sway data (Equation 4-3 and 4-4):

$$
\begin{gathered}
A P[n]=A P_{O}[n]-\overline{A P} \\
M L[n]=M L_{O}[n]-\overline{M L}
\end{gathered}
$$

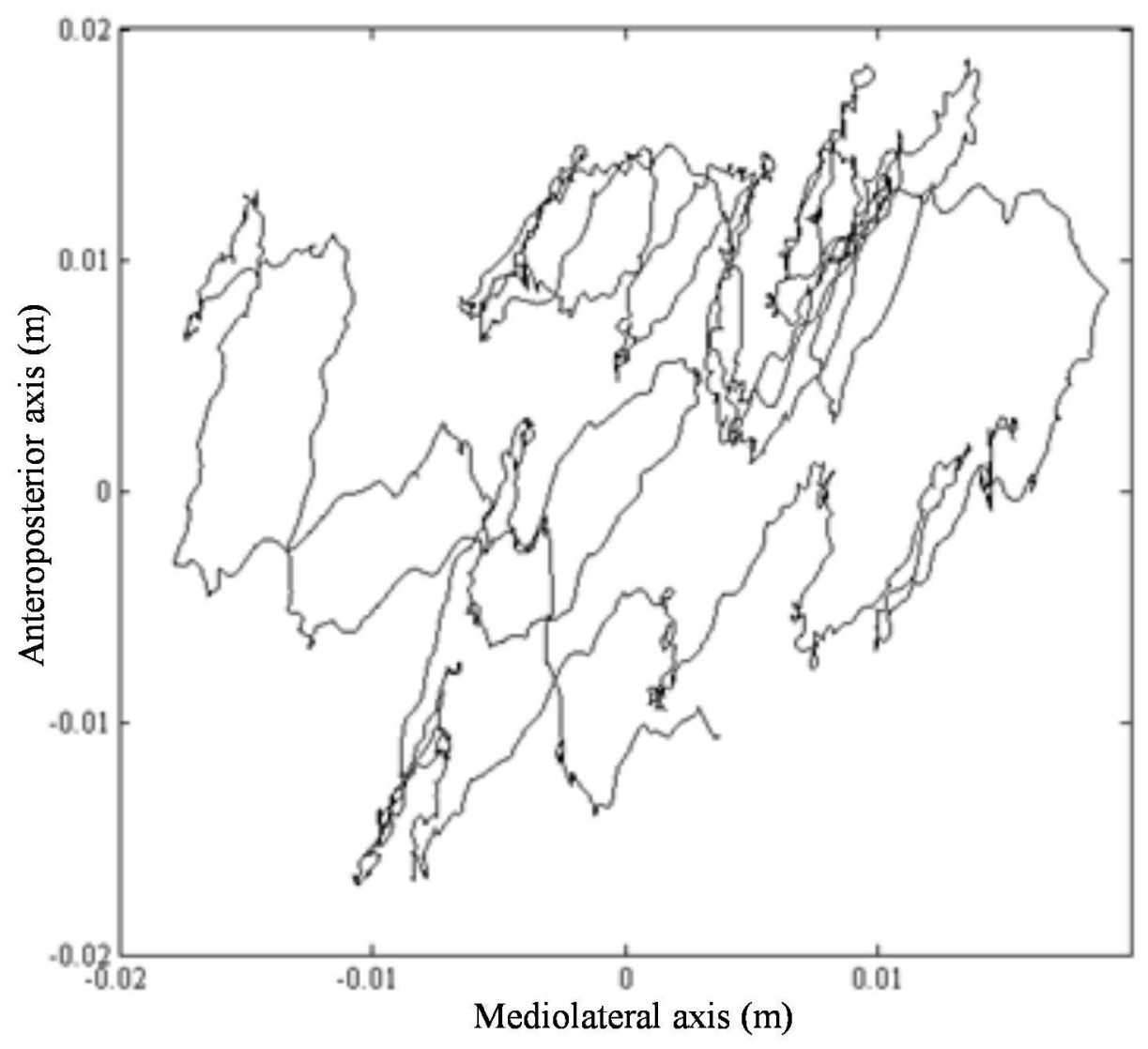

Figure 30: An illustration of the sway motion profile of the motion sensor in one experimental trial 
The motion coordinate time series are commonly used to calculate measures of postural stability and characterize the performance of the postural control system. In principal, these definitions are referenced to the origin of the magnetic field (i.e. transmitter of the motion tracking system). When conducting the experiment, the participant was required to face towards the positive AP direction of the magnetic field. However, since this direction is only approximately aligned with the axes of the magnetic field, measures based on the AP time series probably reflect some ML movements of the participant (vice versa). Therefore, there is a need to introduce a new variable, which is derived from the three dimensional position of sensors but is not sensitive to the orientation of the magnetic field. The resultant distance (RD) time series is the vector distance from the mean sensor position to each pair of points in the $\mathrm{AP}_{\mathrm{O}}$ and MLo time series (Equation 4-5):

$$
R D[n]=\left[A P[n]^{2}+M L[n]^{2}\right]^{1 / 2} n=1, \ldots, N
$$

\section{1) Time-Domain "Distance" Measures}

These are the most commonly used measures of postural stability analysis. Time domain distance measures evaluate a parameter associated with either the displacement of the segment from the center point of the stabilogram, or the velocity of the sensor.

The mean distance (MDIST) is the average of the RD time series and it represents the average distance from the mean of sensor position (Equation 4-6):

$$
M D I S T=1 / N \sum R D[n]
$$


The mean distance-AP (MDIST $\mathrm{AP}_{\mathrm{P}}$ ) is the mean absolute value of the AP time series and represents the average AP distance from the sensor (Equation 4-7):

$$
\operatorname{MDIST}_{A P}=1 / N \sum|A P[n]|
$$

The mean distance-ML (MDIST $\mathrm{ML}_{\mathrm{L}}$ ) is the mean absolute value of the ML time series and represents the average ML distance from the sensor (Equation 4-8):

$$
\operatorname{MDIST}_{M L}=1 / N \sum|M L[n]|
$$

The rms distance (RDIST) from the sensor is the RMS value of the RD time series (Equation 49):

$$
R D I S T=\left[1 / N \sum R D[n]^{2}\right]^{1 / 2}
$$

The rms distance-AP (RDIST ${ }_{\mathrm{AP}}$ ) from the sensor is the standard deviation of the AP time series (Equation 4-10):

$$
\operatorname{RDIST}_{A P}=s_{A P}=\left[1 / N \sum A P[n]^{2}\right]^{1 / 2}
$$

The rms distance-ML (RDIST $\mathrm{ML}$ ) from the sensor is the standard deviation of the ML time series (Equation 4-11):

$$
R D I S T_{M L}=s_{M L}=\left[1 / N \sum M L[n]^{2}\right]^{1 / 2}
$$

The total excursion (TOTEX) is the total length of the sway path and is approximated by the sum of the distances between consecutive points on the sway path (Equation 4-12): 


$$
\text { TOTEX }=\sum_{n=1}^{N-1}\left|(A P[n+1]-A P[n])^{2}+(M L[n+1]-M L[n])^{2}\right|^{1 / 2}
$$

The total excursion-AP (TOTEX $\mathrm{AP}_{\mathrm{AP}}$ ) is the total length of the sensor motion profile in the AP direction, and is approximated by the sum of the distances between consecutive points in the AP time series (Equation 4-13):

$$
\operatorname{TOTEX}_{A P}=\sum_{n=1}^{N-1}|A P[n+1]-A P[n]|
$$

The total excursion-ML (TOTEX $\mathrm{ML}_{\mathrm{L}}$ ) is the total length of the sensor motion profile in the ML direction, and is calculated by the sum of the distances between consecutive points in the ML time series (Equation 4-14):

$$
\operatorname{TOTEX}_{M L}=\sum_{n=1}^{N-1}|M L[n+1]-M L[n]|
$$

The mean velocity (MVELO) is the average velocity of the sway data and it can be divided into $\mathrm{AP}$ and ML directions (MVELO ${ }_{\mathrm{AP}}$ and $\mathrm{MVELO}_{\mathrm{ML}}$ ) (Equation 4-15 to Equation 4-17):

$$
\begin{gathered}
M V E L O=T O T E X / T \\
M V E L O_{A P}=T O T E X_{A P} / T \\
M V E L O_{M L}=T O T E X_{M L} / T
\end{gathered}
$$

2) Time-Domain "Area" Measures

The $95 \%$ confidence circle area (AREA-CC) is the area of a circle with a radius equal to the onesided $95 \%$ confidence limit of the RD time series. Assuming that the distances are normally 
distributed, the AREA-CC models the area of the stabilogram that includes approximately $95 \%$ of the distances from the sensor center (Equation 4-18):

$$
A R E A-C C=\pi\left(M D I S T+z_{0.05} S_{R D}\right)^{2}
$$

$\mathrm{SRD}_{\mathrm{RD}}$ is the standard deviation of the RD time series (Equation 4-19):

$$
S_{R D}=\left[1 / N \sum R D^{2}[n]-M D I S T^{2}\right]^{1 / 2}=\left[R D I S T^{2}-M D I S T^{2}\right]^{1 / 2}
$$

The $95 \%$ confidence ellipse area (AREA-CE) is the area of the $95 \%$ bivariate confidence ellipse, which is expected to enclose approximately $95 \%$ of the points on the sensor location profile. The procedure of obtaining a 95\% confidence ellipse follows previous literature (Sokal and Rohlf 1992). The major radii a and minor radii $b$ of the $95 \%$ confidence ellipse are (Equation 4-20 and Equation 4-21):

$$
\begin{aligned}
& a=\left[F_{.05[2, n-2]}\left(s_{A P}^{2}+s_{M L}^{2}+D\right)\right]^{1 / 2} \\
& a=\left[F_{.05[2, n-2]}\left(s_{A P}^{2}+s_{M L}^{2}-D\right)\right]^{1 / 2}
\end{aligned}
$$

$\mathrm{S}_{\mathrm{AP}}$ and $\mathrm{S}_{\mathrm{ML}}$ are the standard deviation of the AP and ML time series respectively (Equation 4-22):

$$
D=\left[\left(s_{A P}^{2}+s_{M L}^{2}\right)-4\left(s_{A P}^{2} s_{M L}^{2}-s_{A P M L}^{2}\right)\right]^{1 / 2}
$$

Where $\mathrm{s}_{\mathrm{APML}}$ is the covariance (Equation 4-23):

$$
s_{A P M L}=1 / N \sum A P[n] M L[n]
$$

By substituting (4-22) into (4-20) and (4-21), we can simplify the equation (Equation 4-24): 


$$
A R E A-C E=\pi a b=2 \pi F_{.05[2, n-2]}\left[s_{A P}^{2} s_{M L}^{2}-s_{A P M L}^{2}\right]^{1 / 2}
$$

Sway area (AREA-SW) estimates the area enclosed by the sensor position profile per unit of time. This measure is approximated by summing the area of the triangles formed by two consecutive points on the profile and the mean position of the sensor. AREA-SW can be conceptualized as proportional to the product of mean distance and mean velocity (Equation 425):

$$
A R E A-S W=\frac{1}{2 T} \sum_{n=1}^{N-1}|A P[n+1] M L[n]-A P[n] M L[n+1]|
$$

Therefore, for each spinal segment tested in the study (i.e. C7, T12 and L5/S1), there were 15 dependent variables and they are shown in Table 6 .

\subsection{Protocol}

Upon arrival at the work site, the procedure of the experiment was first described to each participant followed by a two-minute warm-up session. Then surface EMG electrodes and motion sensors were attached to the above-described locations using double-sided tape. The skin at placement sites was shaved and cleaned using $70 \%$ alcohol pads. When data collection started, participants were asked to stand on different slanted surfaces with their feet open shoulder width. Markers were placed on the wooden structure to help participants control their foot posture and indicate the direction of the bending motion. The combination of two levels of ASYM and three levels of GROUND generated six different conditions and each condition had four repetitions. Therefore, each participant performed 24 trunk bending trials with the presentation of experiment conditions completely randomized. In each trial, participants were required to 
perform a pace-controlled slow trunk flexion and extension motion with the assistance of a metronome (Figure 31). They were also asked to avoid knee flexion during the task performance in order to eliminate its influence on spinal biomechanical response (Shin et al., 2004). The rhythm of the motion was set as follows: move from an upright standing posture to a fully flexed posture in 7 seconds. When they reach the stable fully flexed status, the experimenter will use a trigger to mark this moment and participants will remain in this posture for another 6 seconds (Figure 32). Finally, they will move back to the upright standing position with another 7 seconds. Two minutes of rest was given between trials in order to avoid the accumulation of lumbar muscle fatigue.

Table 6: Dependent variables in the lumbar stability category

\begin{tabular}{|c|c|c|}
\hline C7 & T12 & L5/S1 \\
\hline C7 MDIST & T12 MDIST & L5 MDIST \\
\hline $\mathrm{C} 7 \mathrm{MDIST}_{\mathrm{AP}}$ & T12 MDIST $_{A P}$ & L5 MDIST $_{A P}$ \\
\hline $\mathrm{C} \mathrm{MDIST}_{\mathrm{ML}}$ & T12 MDIST $_{M L}$ & L5 MDIST $_{M L}$ \\
\hline C7 RDIST & T12 RDIST & L5 RDIST \\
\hline C7 RDIST ${ }_{\mathrm{AP}}$ & T12 RDIST AP & L5 RDIST $_{\mathrm{AP}}$ \\
\hline $\mathrm{C} \mathrm{RDIST}_{\mathrm{ML}}$ & 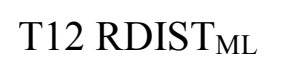 & $\mathrm{L} \mathrm{RDIST}_{\mathrm{ML}}$ \\
\hline C7 TOTEX & T12 TOTEX & L5 TOTEX \\
\hline C7 TOTEX & T12 TOTEX $_{\mathrm{AP}}$ & L5 TOTEX $_{\mathrm{AP}}$ \\
\hline C7 TOTЕХ & T12 TOTEX $\mathrm{ML}$ & L5 TOTEX $\mathrm{ML}$ \\
\hline C7 MVELO & T12 MVELO & L5 MVELO \\
\hline C7 MVELO & T12 MVELO $_{\mathrm{AP}}$ & L5 MVELO $_{\mathrm{AP}}$ \\
\hline C7 MVELOML & T12 MVELOML & L5 MVELOML \\
\hline C7 AREA-CC & T12 AREA-CC & L5 AREA-CC \\
\hline C7 AREA-CE & T12 AREA-CE & L5 AREA-CE \\
\hline C7 AREA-SW & T12 AREA-SW & L5 AREA-SW \\
\hline
\end{tabular}




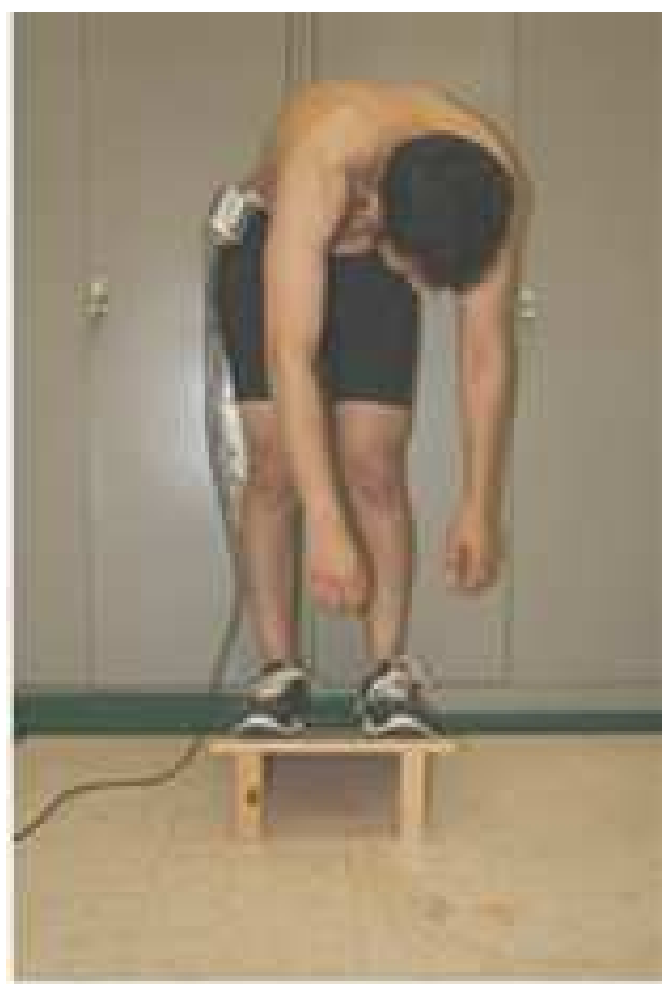

Figure 31: The demonstration of an asymmetric trunk bending task

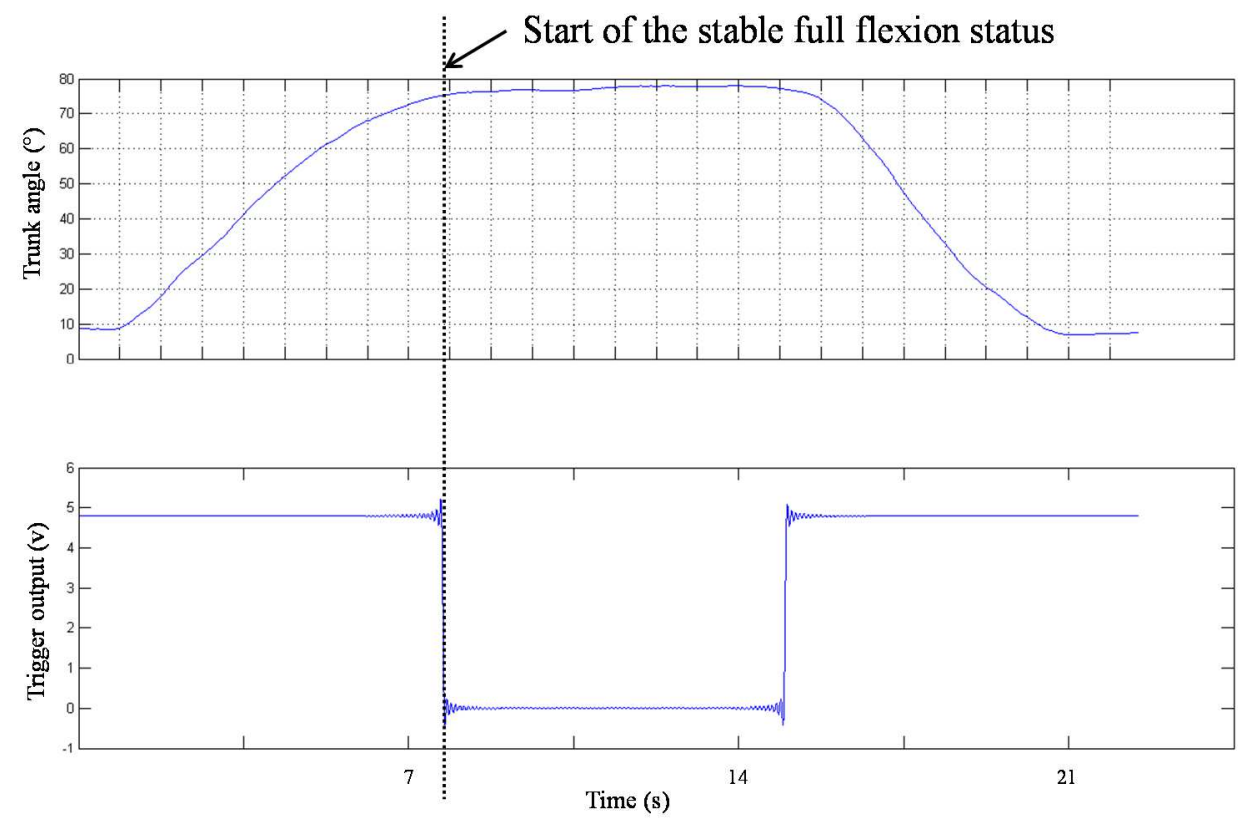

Figure 32: A demonstration of how the trigger was used to determine a fully flexed trunk posture 


\subsection{Data processing}

A customized program (Matlab 2010, MathWorks, USA) was developed to process the EMG and kinematics data. Raw EMG data were transformed into the frequency domain and passed through a $500 \mathrm{~Hz}$ low pass filter, a $10 \mathrm{~Hz}$ high pass filter and a notch filter at $60 \mathrm{~Hz}$ and its aliases. Then, the EMG data were transformed back to the time domain. A 512 points $(0.5$ second) moving window filter was used to generate standard deviation profiles for all sampled lumbar muscles. The EMG-off point was determined with algorithm that used in the pilot study. In the current study, the motion tracking system generated three dimensional coordinates as well as the Euler angle sequence of each motion sensor. The three dimensional coordinates were used to analyze the segmental stability with methods discussed in chapter 4.4. The Eular angle sequence was used to calculate the lumbar flexion angle and trunk inclination angle. Lumbar flexion angle was calculated as the angular difference between the T12 and the S1 sensor in the sagittal plane while the trunk inclination angle was defined as the angle between the vertical line and the line between the $\mathrm{C} 7$ and the $\mathrm{S} 1$ motion sensor.

\subsection{Statistical analysis}

A general linear model was used to perform the statistical analysis:

$$
Y_{i j}=\mu+\tau_{i}+\beta_{j}+\gamma_{k}+(\tau \beta)_{i j}+\varepsilon_{i j k l}
$$

Where $\mathrm{Y}_{\mathrm{ij}}, \mu, \tau_{\mathrm{i}}, \beta_{\mathrm{j}}, \gamma_{\mathrm{k}}, \tau \beta_{\mathrm{ij}}$ and $\varepsilon_{\mathrm{ijkl}}$ represent biomechanical responses (dependent variables), overall mean, main effect of independent variable A, main effect of independent variable B, 
block effect, interaction effect between $\mathrm{A}$ and B, and random error. Total sum of squares was calculated as:

$$
S S_{T}=\sum_{i=1}^{a} \sum_{j=1}^{b} \sum_{k=1}^{c} \sum_{l=1}^{n} Y_{i j k l}^{2}-\frac{y_{\ldots}^{2}}{a b c n}
$$

Sums of squares for the main effects and block effects were found as follows:

$$
\begin{gathered}
S S A=\frac{1}{b c n} \sum_{i=1}^{a} y_{i . . .}^{2}-\frac{y_{\ldots .}^{2}}{a b c n} \\
S S B=\frac{1}{a c n} \sum_{j=1}^{b} y_{. j . .}^{2}-\frac{y_{\ldots}^{2}}{a b c n} \\
S S C=\frac{1}{a b n} \sum_{k=1}^{c} y_{. . k .}^{2}-\frac{y_{\ldots . .}^{2}}{a b c n}
\end{gathered}
$$

Two factors interaction sum of squares was determined as follow:

$$
S S A B=\frac{1}{c n} \sum_{i=1}^{a} \sum_{j=1}^{b} y_{i j . .}^{2}-\frac{y_{\ldots}^{2}}{a b c n}-S S A-S S B
$$

After the assumptions verification procedure, the effects of GROUND, ASYM and their interaction on each dependent variable were then evaluated with repeated measures ANOVA with 'participant' considered as the random factor. To further identify the differences between conditions, Tukey-Kramer post-hoc test was used on the dependent variables that were significantly affected. The criteria p-value was 0.05 for all statistical analysis. All statistical analyses were performed in Minitab (Minitab 17, Minitab Inc., PA., USA). 


\subsection{Results}

The results of FRP analyses showed that when performing sagittally symmetric trunk bending, both sides of the lumbar muscles demonstrated FRP simultaneously. In comparison, when performing asymmetric bending tasks, the contralateral (i.e. to the bending direction) side of lumbar muscles continued to show a high percentage of FRP, while the ipsilateral side of lumbar muscles showed FRP much less frequently (Table 7). These results are in agreement with the findings of a previous study (Ning et al., 2011). Due to the highly unbalanced FRP occurrence rate between the contralateral and ipsilateral side of the lumbar muscles, only the contralateral side of lumbar muscles was further analyzed.

The results of ANOVA showed that ASYM significantly affected all dependent variables, while GROUND significantly influenced all the dependent variables except the MTI angle (Table 8). The full ANOVA table can be found in Appendix A. 
Table 7: Percentage of trials that showed FRP among the sampled lumbar muscles

\begin{tabular}{cccc}
\hline & \multicolumn{3}{c}{ Erector Spinae (\%) } \\
\cline { 2 - 4 } Asymmetry & Ground & Left & Right \\
\hline Symmetric & 15 & 100 & 94.6 \\
& 0 & 91.1 & 94.7 \\
\hline Asymmetric & -15 & 96.4 & 100 \\
& 15 & 55.3 & 94.6 \\
& 0 & 67 & 94.7 \\
& -15 & 75 & 98.2 \\
\hline Asymmetry & & & \\
\hline Symmetric & & Multifidus $(\%)$ & Right \\
\hline Asymmetric & Ground & Left & 82.1 \\
& 15 & 89.3 & 94.6 \\
\hline & 0 & 94.6 & 94.6 \\
& -15 & 100 & 85.7 \\
& 15 & 80.4 & 92.9 \\
& 0 & 83.9 & 82.1 \\
\hline & -15 & 78.6 & \\
\hline
\end{tabular}

Table 8: The results of univariate ANOVA on FRP related variables

\begin{tabular}{llllllll}
\hline & \multicolumn{7}{c}{ ANOVA } \\
\cline { 2 - 7 } $\begin{array}{l}\text { Independent } \\
\text { variables }\end{array}$ & MLF & MTI & RL3L & RL3T & RL4L & RL4T \\
\hline ASYM & $<\mathbf{0 . 0 0 1}$ & $<\mathbf{0 . 0 0 1}$ & $<\mathbf{0 . 0 0 1}$ & $<\mathbf{0 . 0 0 1}$ & $<\mathbf{0 . 0 0 1}$ & $<\mathbf{0 . 0 0 1}$ \\
GROUND & $\mathbf{0 . 0 2 9}$ & 0.328 & $\mathbf{0 . 0 1 5}$ & $<\mathbf{0 . 0 0 1}$ & $\mathbf{0 . 0 0 3}$ & $<\mathbf{0 . 0 0 1}$ \\
ASYM*GROUND & 0.111 & $\mathbf{0 . 0 4 9}$ & 0.277 & 0.263 & 0.098 & $\mathbf{0 . 0 3 1}$ \\
\hline
\end{tabular}

Notes: MLF = maximum lumbar flexion angle; MTI = maximum trunk inclination angle; RL3L = right L3 paraspinal EMG-off lumbar angle; RL3T = right L3 paraspinal EMG-off trunk angle; RL4L = right L4 paraspinal EMG-off lumbar angle; RL4T = right L4 paraspinal EMG-off trunk angle. 
During asymmetric bending, significantly reduced EMG-off lumbar flexion angle and trunk inclination angle was observed on the contralateral side of lumbar muscles, as compared to the symmetric condition. In addition, participants demonstrated significantly smaller maximum lumbar flexion angle and MTI angle (Figure 33 to Figure 38).

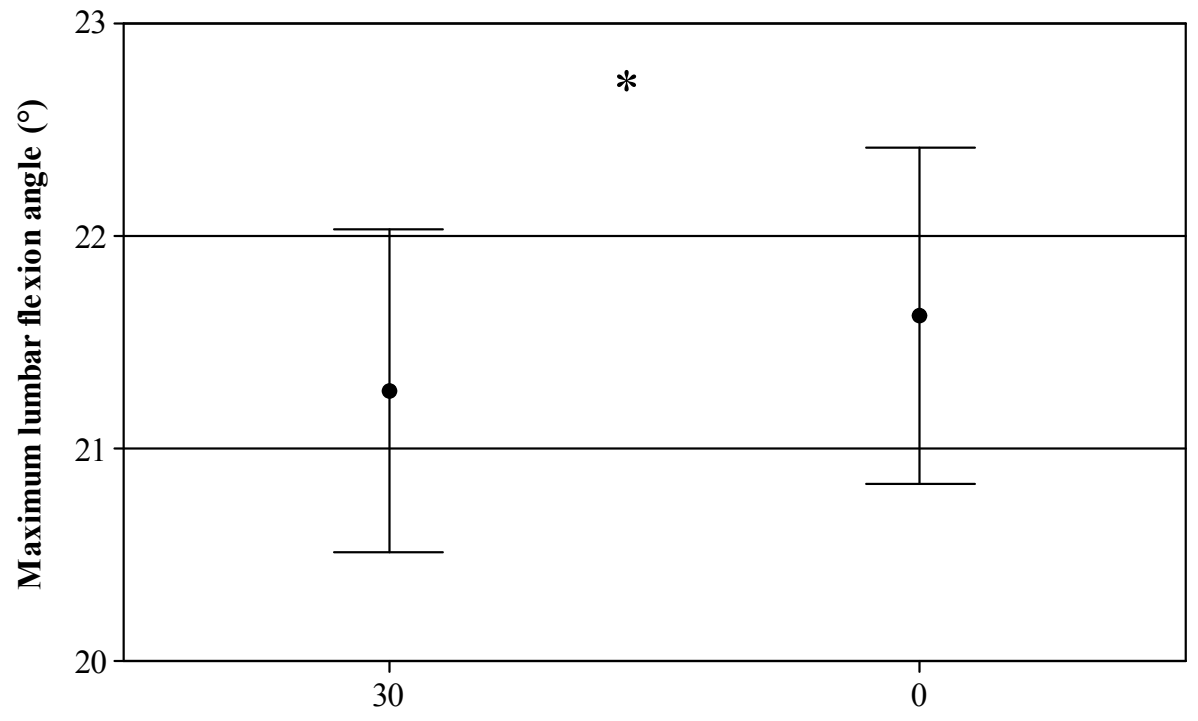

Figure 33: The effect of ASYM on maximum lumbar flexion angle (MLF) 


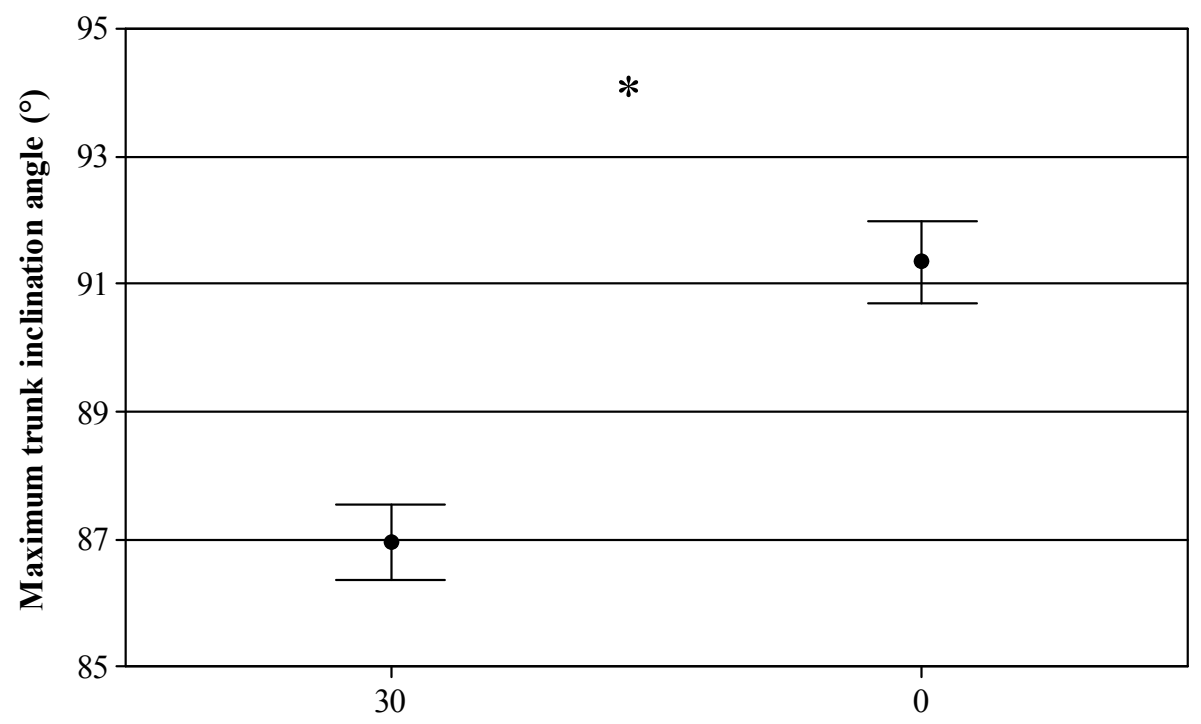

Figure 34: The effect of ASYM on maximum trunk inclination angle (MTI)

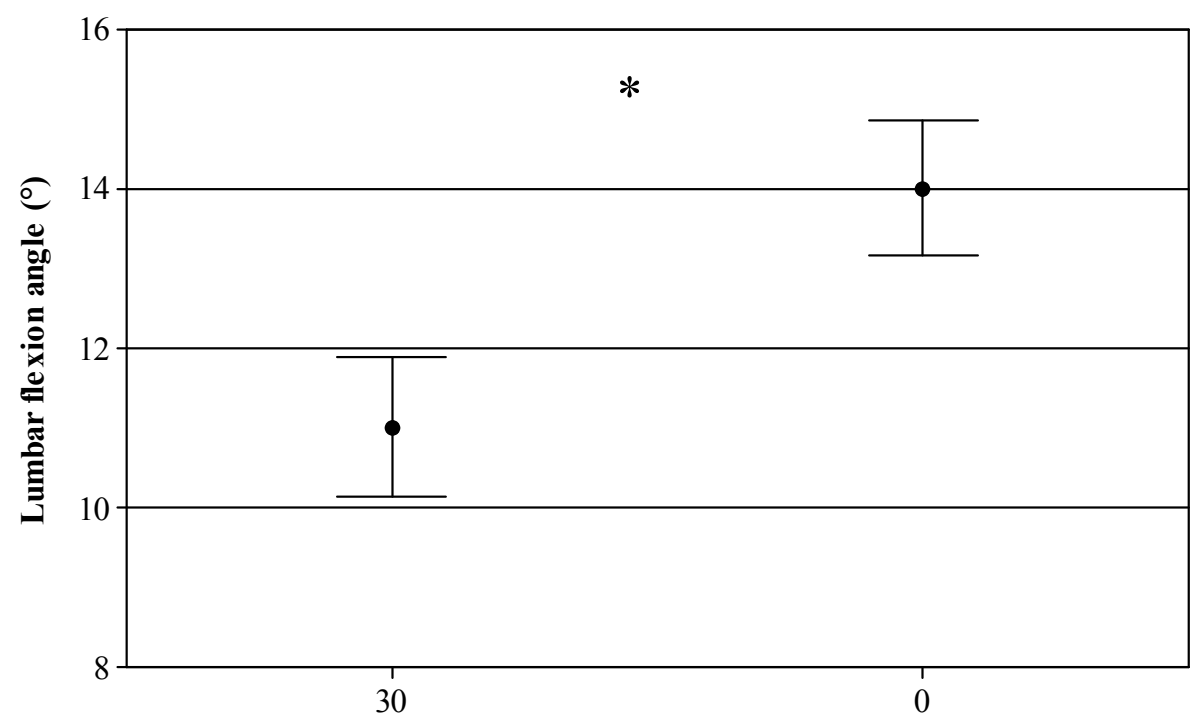

Figure 35: The effect of ASYM on right erector spinae EMG-off lumbar angle (RL3L) 


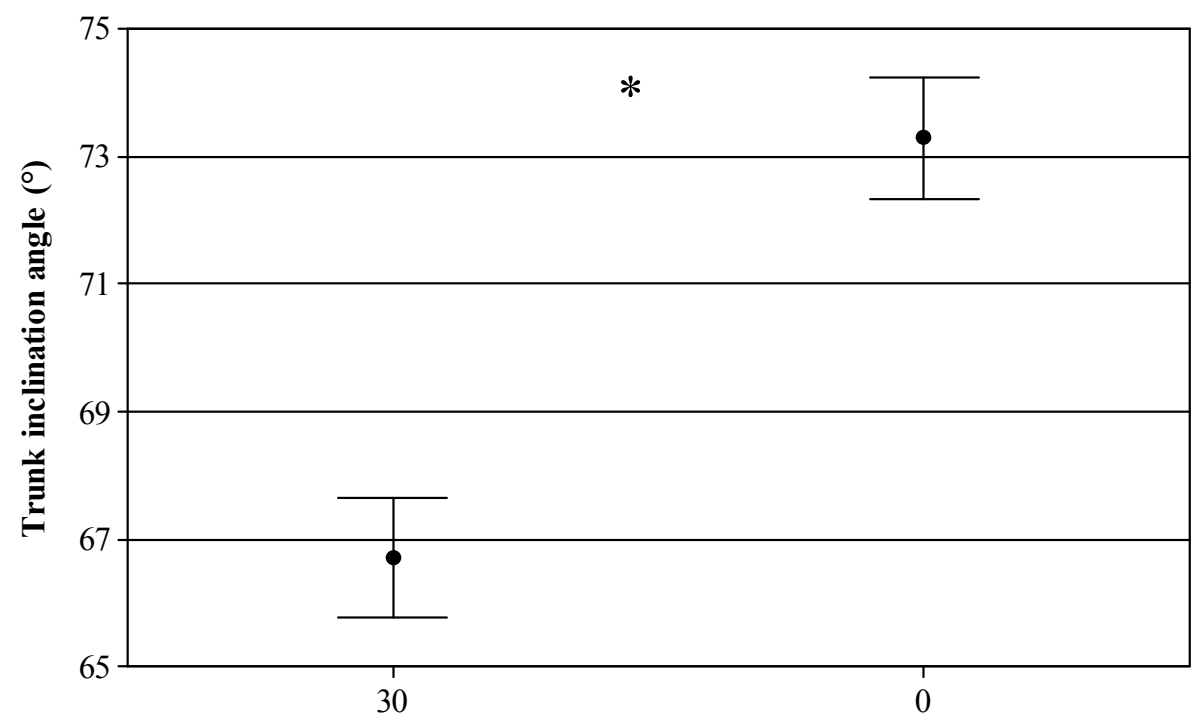

Figure 36: The effect of ASYM on right erector spinae EMG-off trunk angle (RL3T)

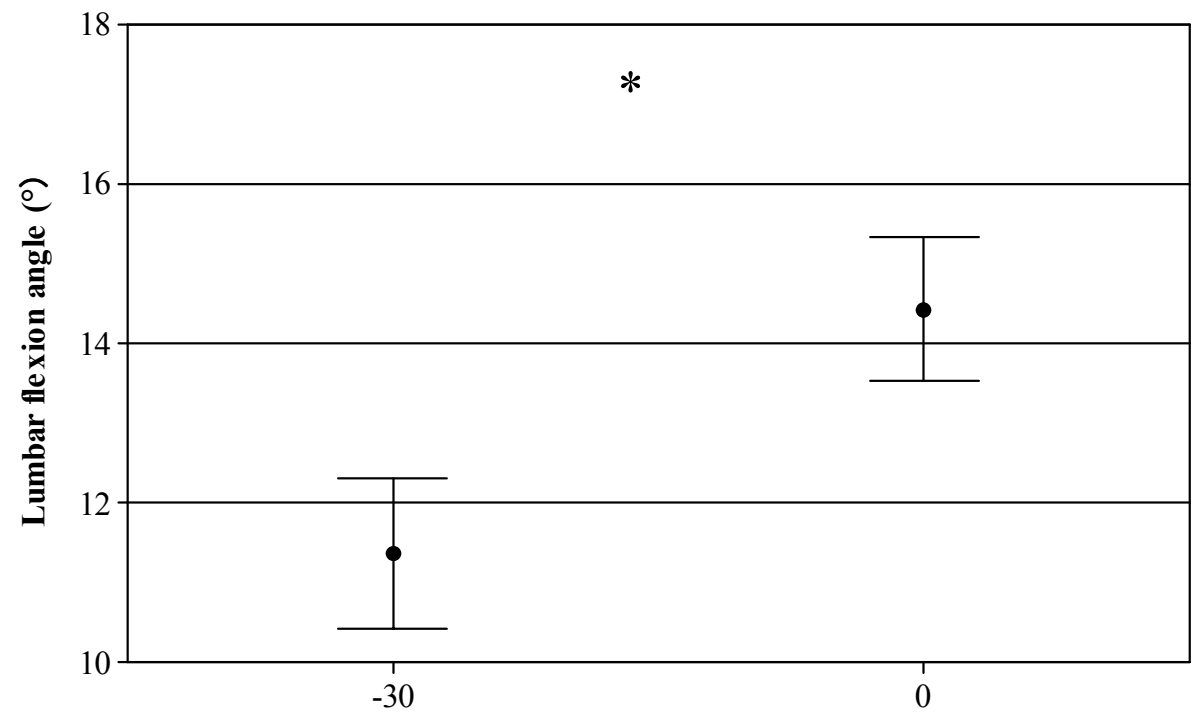

Figure 37: The effect of ASYM on right multifidus EMG-off lumbar angle (RL4L) 


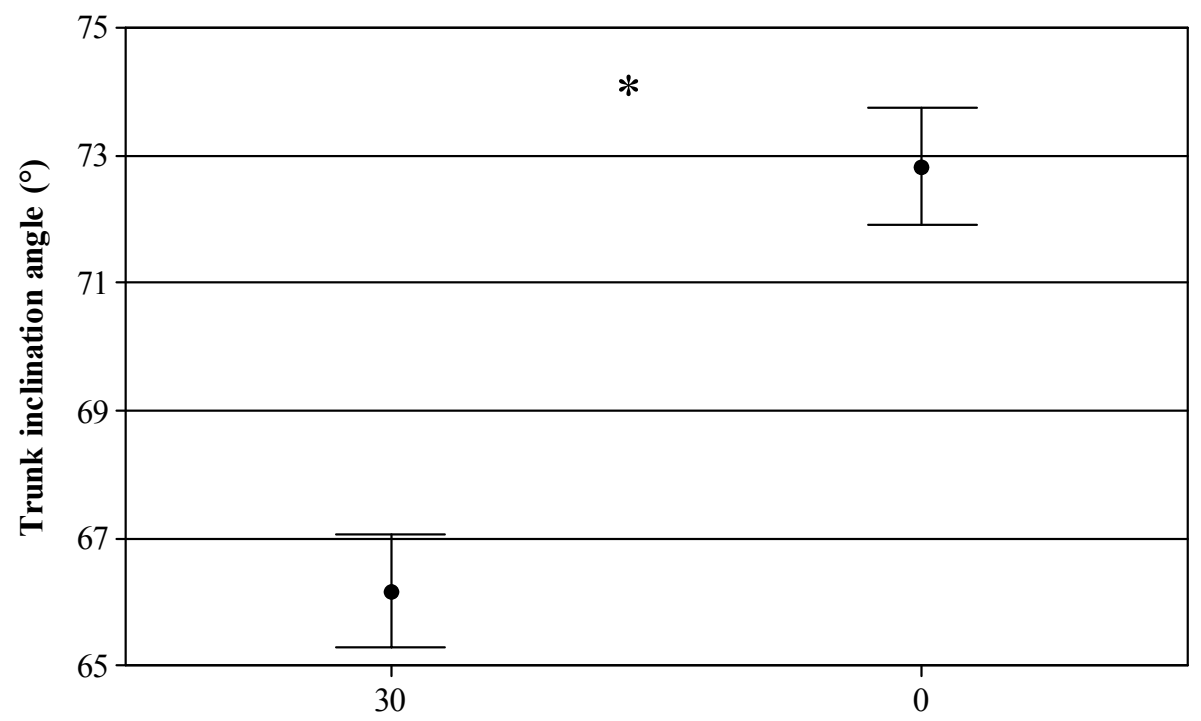

Figure 38: The effect of ASYM on right multifidus EMG-off trunk angle (RL4T)

Ground had strong influence on all FRP related variables. Specifically, standing on the - 15 uphill facing surface resulted in smaller EMG-off lumbar flexion angle and trunk inclination angle, as compared to the flat ground condition. Standing on the 15 downhill facing surface resulted in larger EMG-off angles (Figure 39 to Figure 42). In addition, larger maximum lumbar flexion angle was observed in the slanted ground conditions, especially in the 15 condition (Figure 43). Finally, the interaction effect of GROUND and ASYM was significant on the MTI angle (Figure 44). 


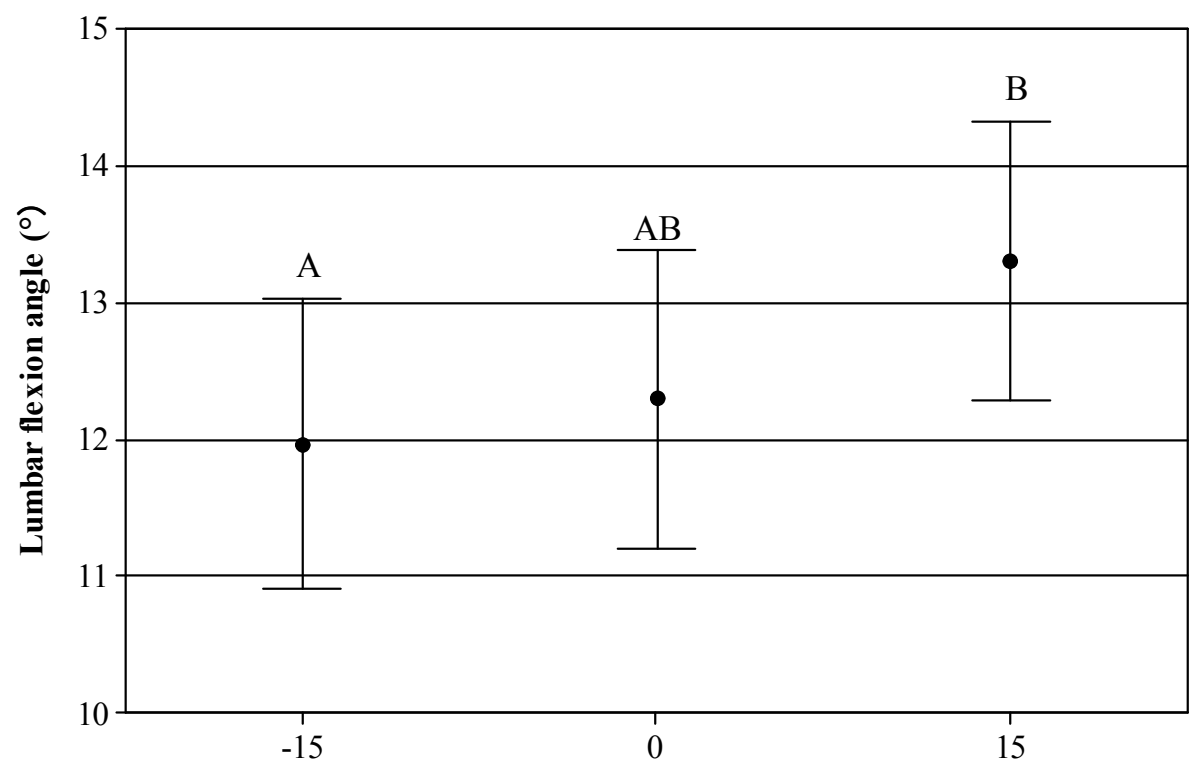

Figure 39: The effect of GROUD on right erector spinae EMG-off lumbar angle (RL3L)

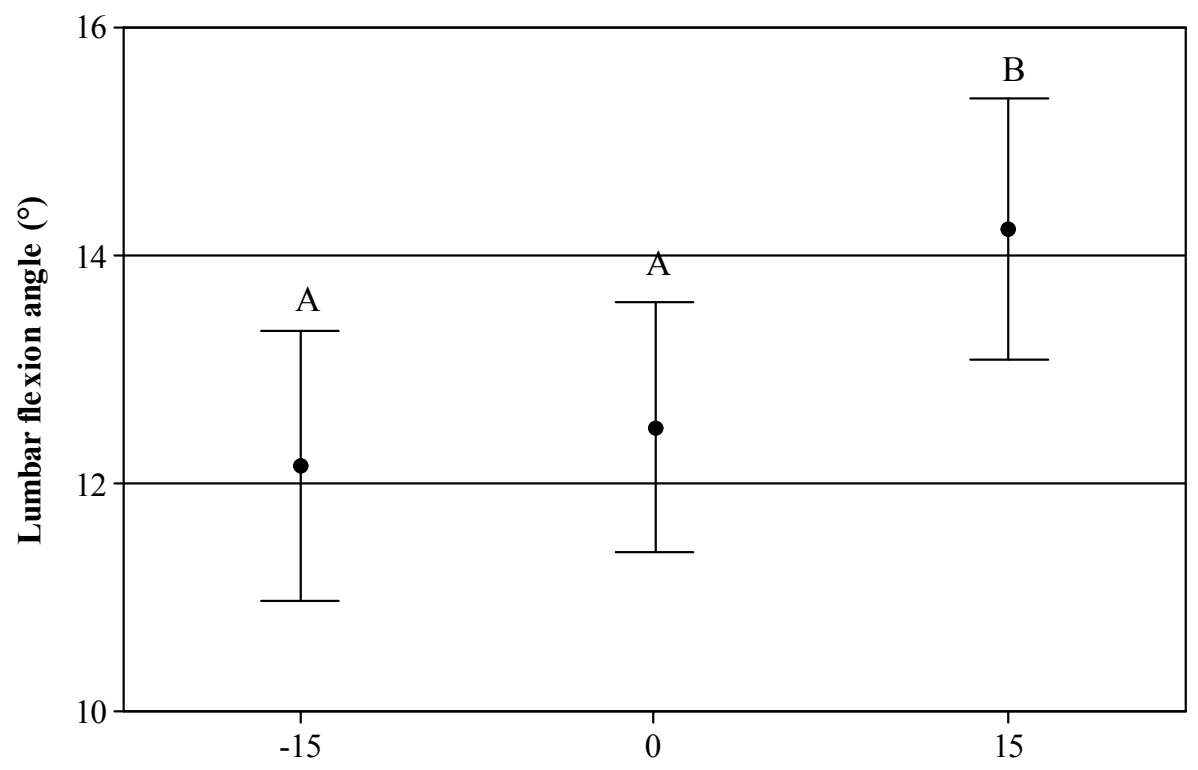

Figure 40: The effect of GROUD on right multifidus EMG-off lumbar angle (RL4L) 


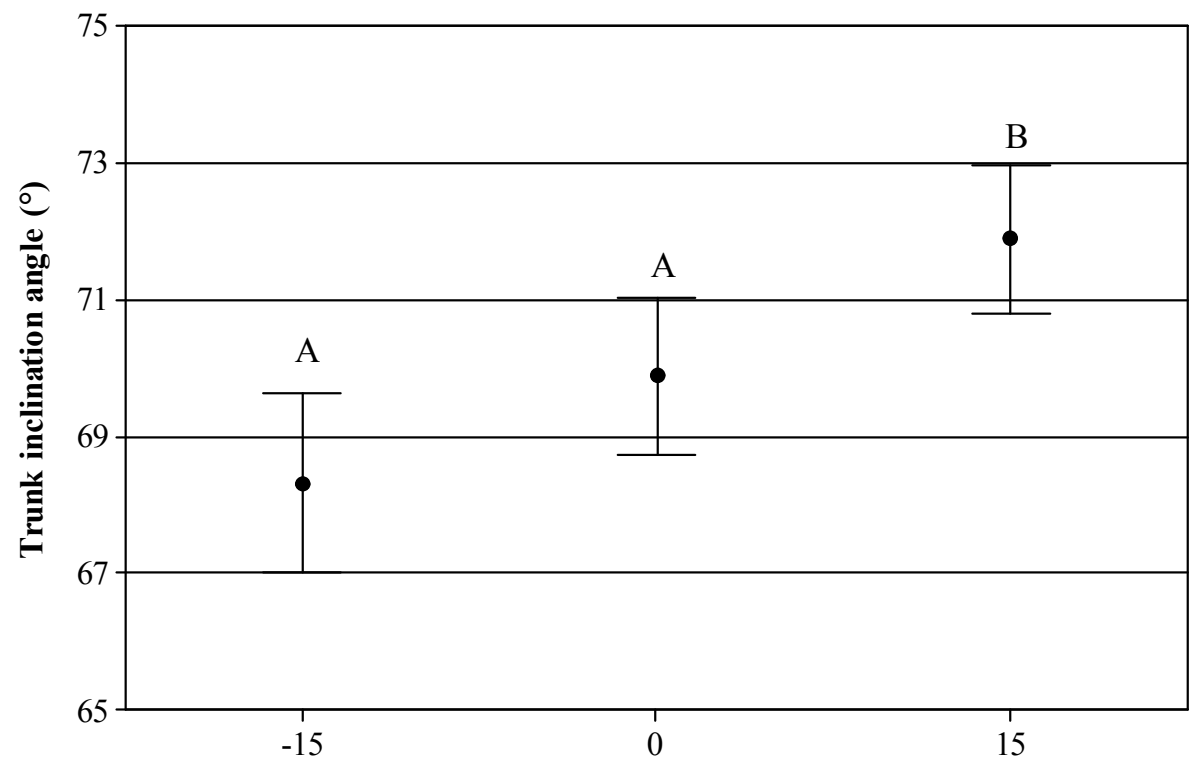

Figure 41: The effect of GROUD on right erector spinae EMG-off trunk angle (RL4L)

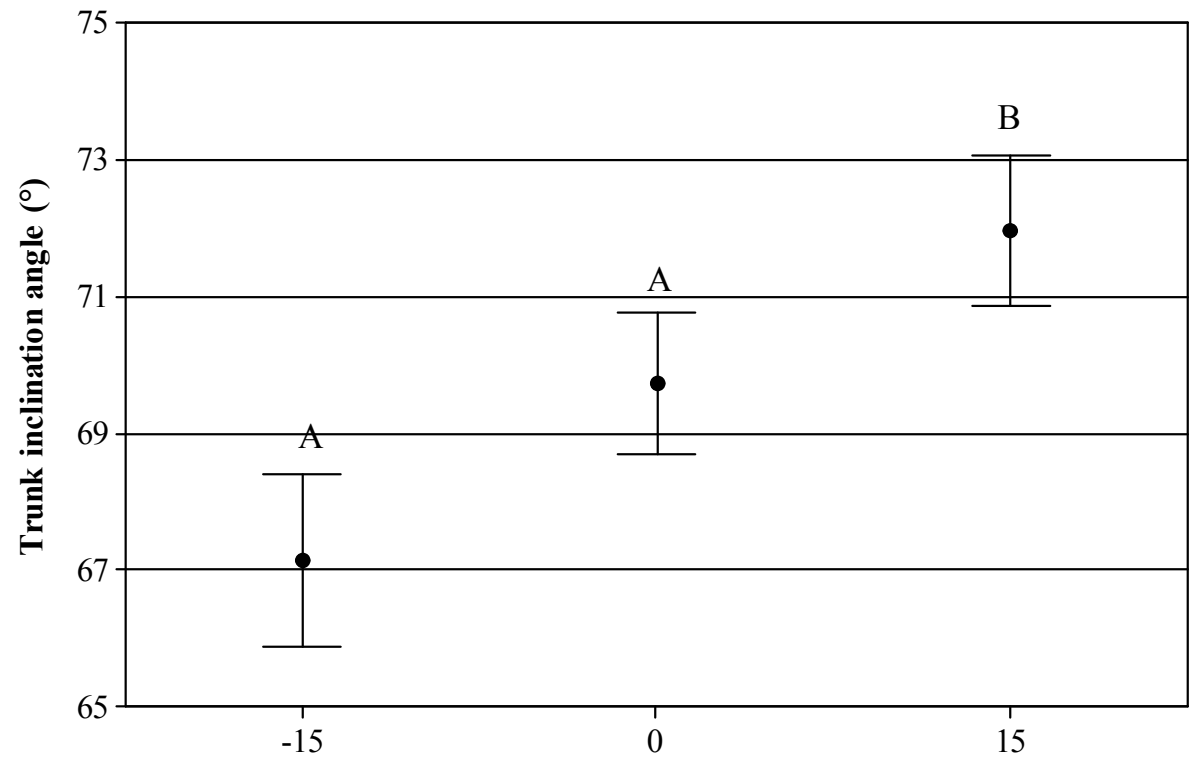

Figure 42: The effect of GROUD on right multifidus EMG-off trunk angle (RL4L) 


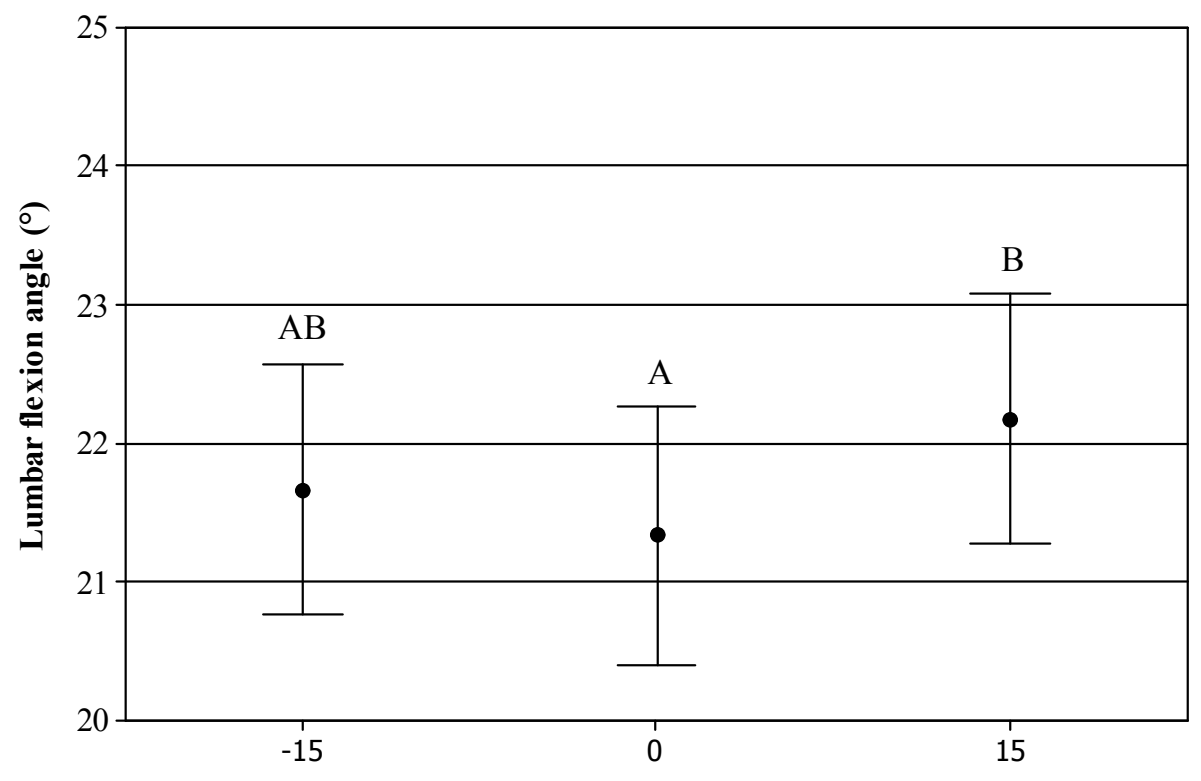

Figure 43: Effects of GROUND on maximum lumbar flexion angle

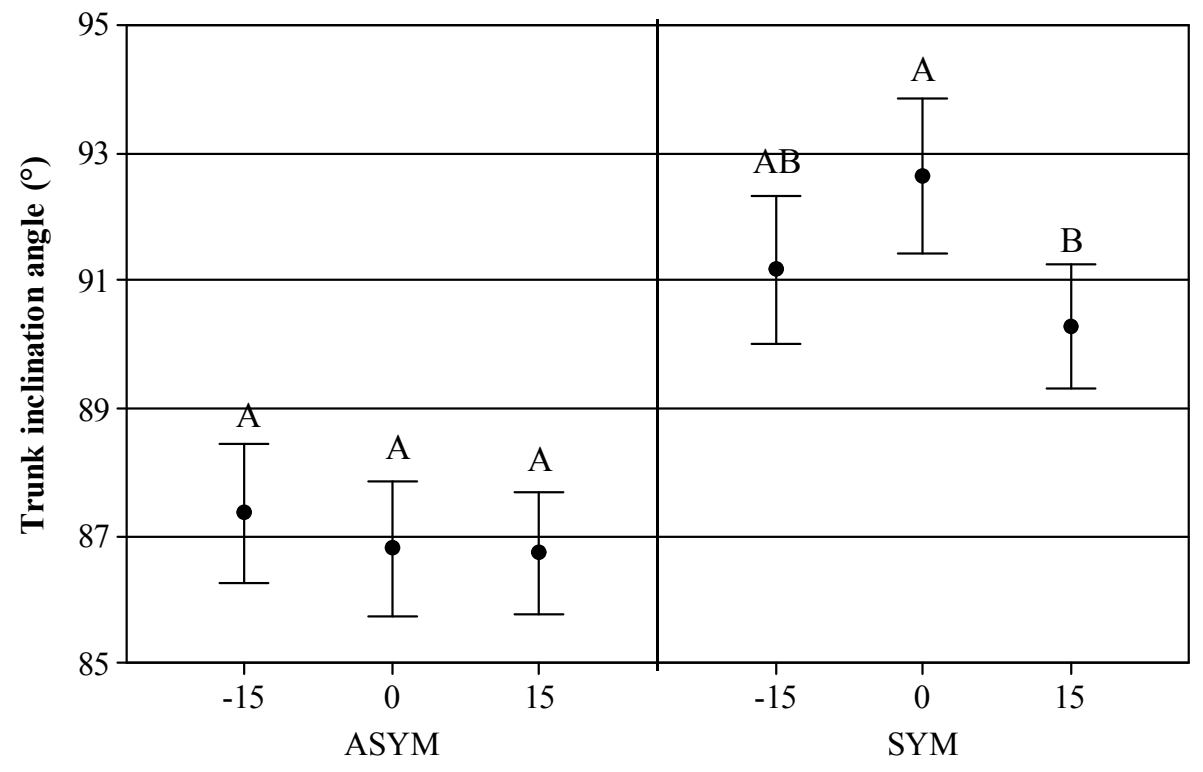

Figure 44: Effects of GROUND on MTI angle under different ASYM conditions

For the lumbar segmental stability analysis, no significant interaction effects on any dependent variable were found (Table 9 to Table 11). Full ANOVA table can be found in Appendix A. 
Therefore, no further analysis was performed to explore the influence of interaction effects in the current research.

Table 9: The results of univariate ANOVA on C7 segmental stability variables

\begin{tabular}{|c|c|c|c|c|c|c|c|c|}
\hline $\mathrm{C} 7$ & MDIST & $\begin{array}{c}\text { MDIST } \\
\text { AP }\end{array}$ & $\begin{array}{c}\text { MDIST } \\
\text { ML }\end{array}$ & $\begin{array}{c}\text { RDIST } \\
\text { C7 }\end{array}$ & $\begin{array}{c}\text { RDIST } \\
\text { AP }\end{array}$ & $\begin{array}{c}\text { RDIST } \\
\text { ML }\end{array}$ & TOTEX & $\begin{array}{c}\text { TOTEX } \\
\text { AP }\end{array}$ \\
\hline GROUND & 0.146 & 0.237 & 0.122 & 0.151 & 0.279 & 0.105 & $<0.001$ & 0.004 \\
\hline ASYM & $<0.001$ & $<0.001$ & 0.16 & $<0.001$ & $<0.001$ & 0.151 & 0.933 & 0.028 \\
\hline GROUND*ASYM & 0.509 & 0.307 & 0.578 & 0.637 & 0.297 & 0.723 & 0.254 & 0.199 \\
\hline $\mathrm{C} 7$ & $\begin{array}{c}\text { TOTEX } \\
\text { ML }\end{array}$ & MVELO & $\begin{array}{r}\mathrm{MVH} \\
\mathrm{A}\end{array}$ & & $\begin{array}{l}\text { MVELO } \\
\text { ML }\end{array}$ & $\begin{array}{c}\text { AREA } \\
\text { CC }\end{array}$ & $\begin{array}{c}\text { AREA } \\
\text { CE }\end{array}$ & $\begin{array}{c}\text { AREA } \\
\text { SW }\end{array}$ \\
\hline GROUND & $<0.001$ & $<0.001$ & 0.0 & & $<0.001$ & 0.228 & 0.278 & 0.158 \\
\hline ASYM & 0.025 & 0.937 & 0.0 & & 0.025 & 0.001 & $<0.001$ & 0.012 \\
\hline GROUND*ASYM & 0.403 & 0.247 & 0.1 & & 0.398 & 0.162 & 0.137 & 0.669 \\
\hline
\end{tabular}

Table 10: The results of univariate ANOVA on T12 segmental stability variables

\begin{tabular}{|c|c|c|c|c|c|c|c|c|}
\hline $\mathrm{T} 12$ & MDIST & $\begin{array}{c}\text { MDIST } \\
\text { AP }\end{array}$ & $\begin{array}{c}\text { MDIST } \\
\text { ML }\end{array}$ & RDIST & $\begin{array}{c}\text { RDIST } \\
\text { AP }\end{array}$ & $\begin{array}{c}\text { RDIST } \\
\text { ML }\end{array}$ & TOTEX & $\begin{array}{c}\text { TOTEX } \\
\text { AP }\end{array}$ \\
\hline GROUND & 0.129 & 0.71 & 0.131 & 0.165 & 0.583 & 0.098 & $<0.001$ & 0.002 \\
\hline ASYM & 0.393 & 0.001 & 0.397 & 0.009 & $<0.001$ & 0.65 & 0.878 & $<0.001$ \\
\hline GROUND*ASYM & 0.94 & 0.525 & 0.633 & 0.951 & 0.312 & 0.747 & 0.104 & 0.22 \\
\hline $\mathrm{T} 12$ & $\begin{array}{c}\text { TOTEX } \\
\text { ML }\end{array}$ & MVELO & $\begin{array}{c}\text { MVELO } \\
\text { AP }\end{array}$ & & $\begin{array}{l}\mathrm{ELO} \\
\mathrm{ML}\end{array}$ & $\begin{array}{c}\text { AREA } \\
\mathrm{CC} \\
\end{array}$ & $\begin{array}{c}\text { AREA } \\
\text { CE }\end{array}$ & $\begin{array}{c}\text { AREA } \\
\text { SW }\end{array}$ \\
\hline GROUND & $<0.001$ & $<0.001$ & 0.002 & & .001 & 0.575 & 0.843 & 0.276 \\
\hline ASYM & 0.059 & 0.886 & $<0.001$ & & 059 & 0.025 & 0.001 & 0.067 \\
\hline GROUND*ASYM & 0.156 & 0.104 & 0.218 & & 156 & 0.582 & 0.539 & 0.699 \\
\hline
\end{tabular}


Table 11: The results of univariate ANOVA on S1 segmental stability variables

\begin{tabular}{lcccccccc}
\hline \multicolumn{1}{c}{ S1 } & \multirow{2}{*}{ MDIST } & $\begin{array}{c}\text { MDIST } \\
\text { AP }\end{array}$ & $\begin{array}{c}\text { MDIST } \\
\text { ML }\end{array}$ & RDIST & $\begin{array}{c}\text { RDIST } \\
\text { AP }\end{array}$ & $\begin{array}{c}\text { RDIST } \\
\text { ML }\end{array}$ & TOTEX & $\begin{array}{c}\text { TOTEX } \\
\text { AP }\end{array}$ \\
\hline GROUND & 0.055 & $\mathbf{0 . 0 3 1}$ & 0.353 & 0.204 & 0.633 & 0.141 & $<\mathbf{0 . 0 0 1}$ & $<\mathbf{0 . 0 0 1}$ \\
ASYM & 0.904 & $\mathbf{0 . 0 0 8}$ & 0.164 & $\mathbf{0 . 0 3 2}$ & $<\mathbf{0 . 0 0 1}$ & 0.912 & 0.215 & $<\mathbf{0 . 0 0 1}$ \\
GROUND*ASYM & 0.773 & 0.947 & 0.624 & 0.991 & 0.367 & 0.707 & 0.104 & 0.116 \\
\hline \multicolumn{1}{c}{ S1 } & & & & & & & & \\
& TOTEX & MVELO & MVELO & MVELO & AREA & AREA & AREA \\
& ML & MP & ML & CC & CE & SW \\
\hline GROUND & $<\mathbf{0 . 0 0 1}$ & $<\mathbf{0 . 0 0 1}$ & $<\mathbf{0 . 0 0 1}$ & $\mathbf{0 . 0 0 1}$ & 0.746 & 0.881 & $\mathbf{0 . 0 5}$ \\
ASYM & 0.57 & 0.217 & $<\mathbf{0 . 0 0 1}$ & 0.572 & 0.077 & $\mathbf{0 . 0 0 2}$ & 0.113 \\
GROUND*ASYM & 0.249 & 0.103 & 0.114 & 0.246 & 0.751 & 0.671 & 0.312 \\
\hline
\end{tabular}

ASYM demonstrated statistically significant effects on participants' lumbar segmental stability performance at all three segments $(\mathrm{C} 7, \mathrm{~T} 12$ and $\mathrm{S} 1)$. In general, in asymmetric conditions, participants showed larger sway distance, sway velocity and sway area (Table 12 to Table 14). Specifically, all three segments had significantly larger MDIST and RDIST in the anteroposterior direction while virtually no changes were found in the medio-lateral direction. Participants also showed larger sway velocity in the asymmetric condition, especially in the antero-posterior direction. In addition, larger sway areas were found in the asymmetric condition. However, three segments did not show the same pattern. C7 segment had larger AREA CC, AREA CE and AREA SW in the asymmetric condition, while S1 segment only showed larger AREA CE. 
Table 12: Effect of ASYM on C7 segmental stability

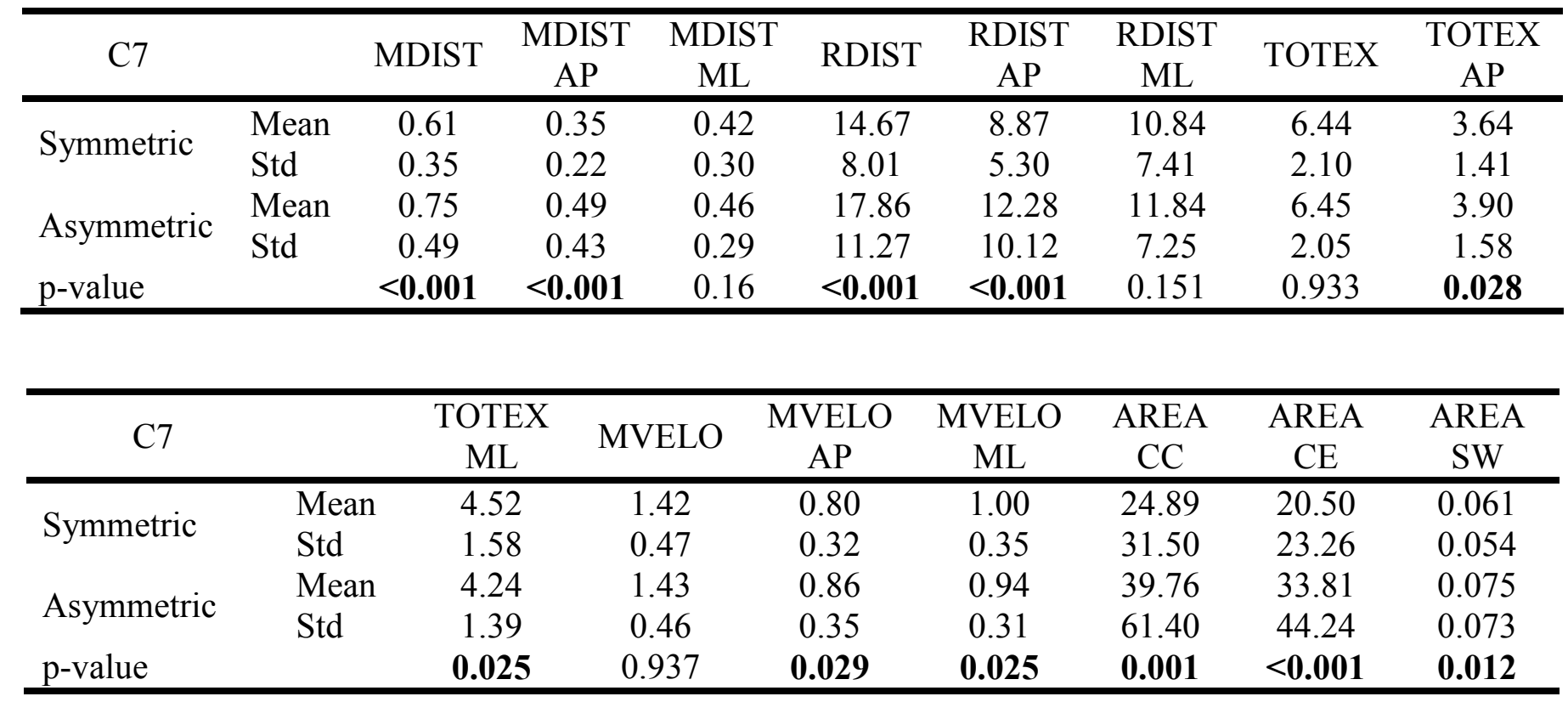

Table 13: Effect of ASYM on T12 segmental stability

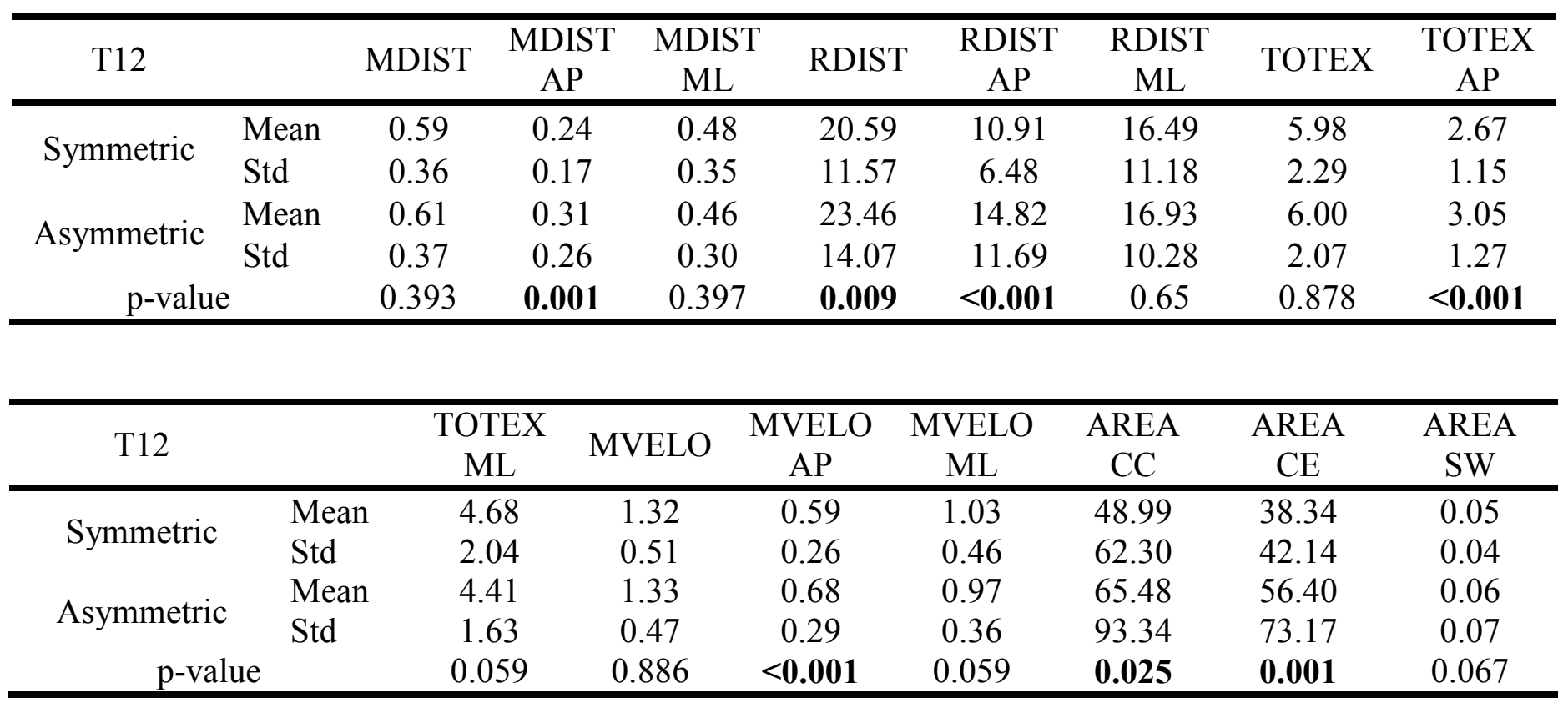


Table 14: Effect of ASYM on S1 segmental stability

\begin{tabular}{|c|c|c|c|c|c|c|c|c|c|}
\hline $\mathrm{S} 1$ & & MDIST & $\begin{array}{c}\text { MDIST } \\
\text { AP }\end{array}$ & $\begin{array}{c}\text { MDIST } \\
\text { ML }\end{array}$ & RDIST & $\begin{array}{c}\text { RDIST } \\
\text { AP }\end{array}$ & $\begin{array}{c}\text { RDIST } \\
\text { ML }\end{array}$ & TOTEX & $\begin{array}{c}\text { TOTEX } \\
\text { AP }\end{array}$ \\
\hline \multirow{2}{*}{ Symmetric } & Mean & 0.48 & 0.20 & 0.40 & 23.76 & 12.22 & 19.36 & 5.31 & 2.47 \\
\hline & Std & 0.30 & 0.14 & 0.29 & 13.38 & 7.14 & 13.00 & 1.83 & 1.05 \\
\hline \multirow{2}{*}{ Asymmetric } & Mean & 0.49 & 0.24 & 0.36 & 26.39 & 16.31 & 19.48 & 5.47 & 2.78 \\
\hline & Std & 0.28 & 0.21 & 0.21 & 15.28 & 12.45 & 11.39 & 1.77 & 1.12 \\
\hline \multicolumn{2}{|c|}{ p-value } & 0.904 & 0.008 & 0.164 & 0.032 & $<0.001$ & 0.912 & 0.215 & $<0.001$ \\
\hline \multicolumn{2}{|l|}{$\mathrm{S} 1$} & $\begin{array}{c}\text { TOTEX } \\
\text { ML }\end{array}$ & MVELO & $\begin{array}{c}\text { MVELO } \\
\text { AP }\end{array}$ & \multicolumn{2}{|c|}{$\begin{array}{c}\text { MVELO } \\
\text { ML }\end{array}$} & $\begin{array}{c}\text { AREA } \\
\text { CC }\end{array}$ & $\begin{array}{c}\text { AREA } \\
\text { CE }\end{array}$ & AREA SW \\
\hline \multirow{2}{*}{ Symmetric } & Mean & 4.08 & 1.17 & 0.55 & \multicolumn{2}{|c|}{0.90} & 64.72 & $\begin{array}{l}50.78 \\
5551\end{array}$ & 0.04 \\
\hline & Std & 1.56 & 0.41 & 0.24 & \multicolumn{2}{|c|}{0.35} & 83.73 & 55.51 & 0.03 \\
\hline \multirow{2}{*}{ Asymmetric } & Mean & 4.02 & 1.21 & 0.61 & \multicolumn{2}{|c|}{0.89} & 80.65 & 71.58 & 0.04 \\
\hline & Std & 1.32 & 0.40 & 0.25 & \multicolumn{2}{|c|}{0.30} & 110.79 & 92.24 & 0.05 \\
\hline \multicolumn{2}{|c|}{$\mathrm{p}$-value } & 0.57 & 0.217 & $<0.001$ & \multicolumn{2}{|c|}{0.572} & 0.077 & 0.002 & 0.113 \\
\hline
\end{tabular}

The results showed that GROUND demonstrated more subtle effects on participants' lumbar segmental stability performance when they maintained a static deep bending posture. In general, participants showed worse segmental stability in the uphill facing condition (Figure 45 to Figure 64). To be specific, Figures 45 to Figure 50 show significant variables at the C7 level; Figures 51 to Figure 56 show significant variables at the T12 level while Figures 57 to Figure 64 show significant variables at the S1 level. Among all three segments, MDIST and RDIST were not affected by GROUND except S1 MDIST AP. However, TOTEX and sway velocity at all three segments were significantly affected by GROUND. In terms of sway area variables, only AREA SW at the S1 was significantly affected by GROUND. Finally, it is important to note that compared to $\mathrm{C} 7$ and $\mathrm{T} 12$ segments, $\mathrm{S} 1$ demonstrated the worst stability performance. In addition, figures for insignificant results are showing in the Appendix C. 


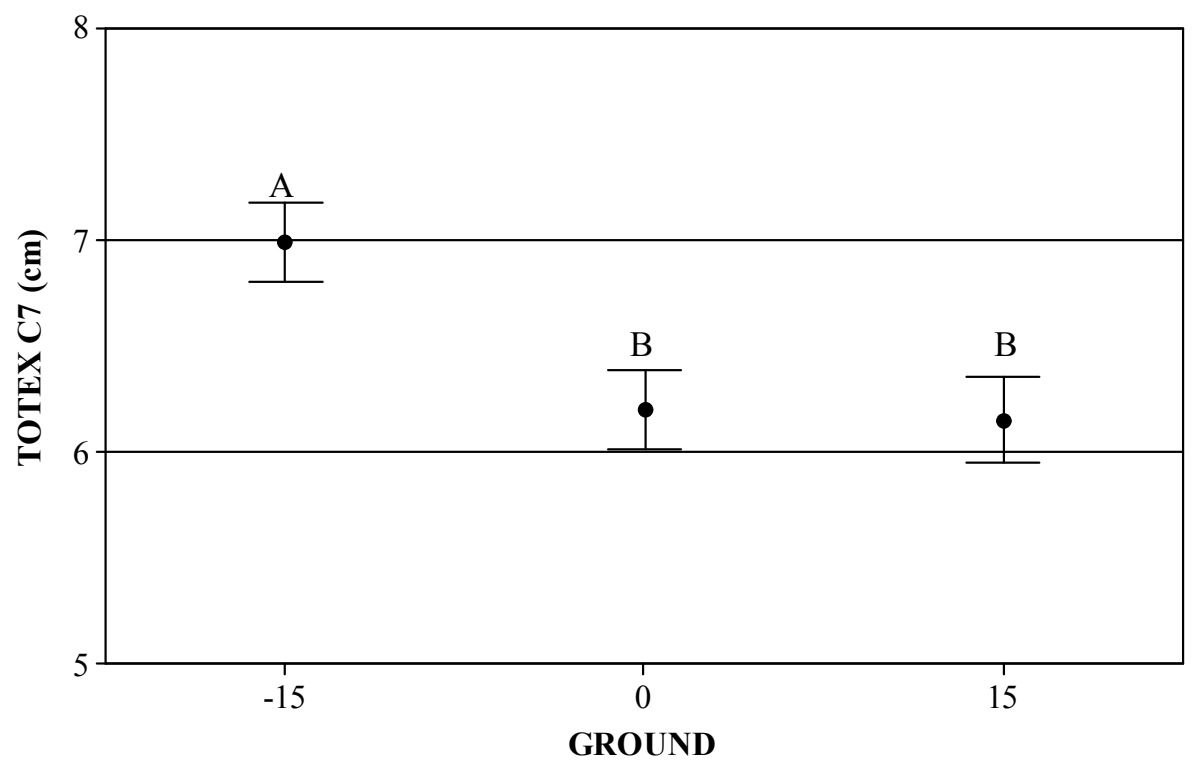

Figure 45: Effects of GROUND on C7 TOTEX

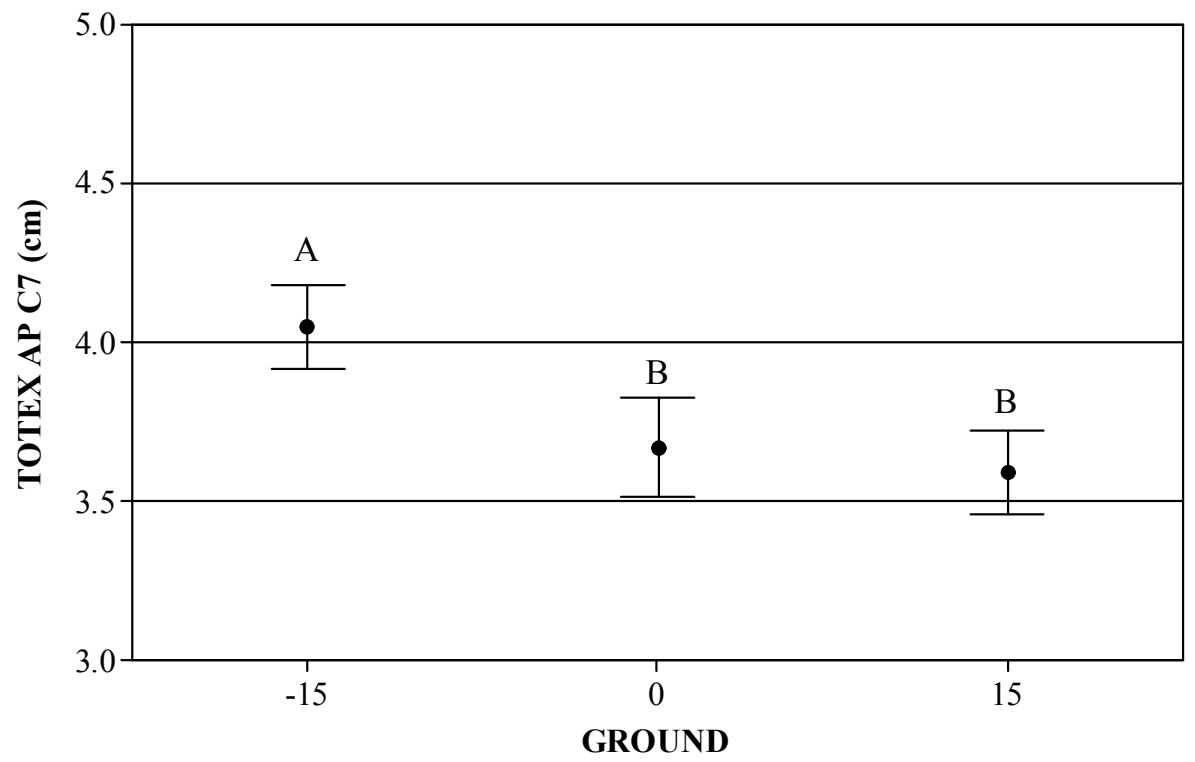

Figure 46: Effects of GROUND on C7 TOTEX AP 


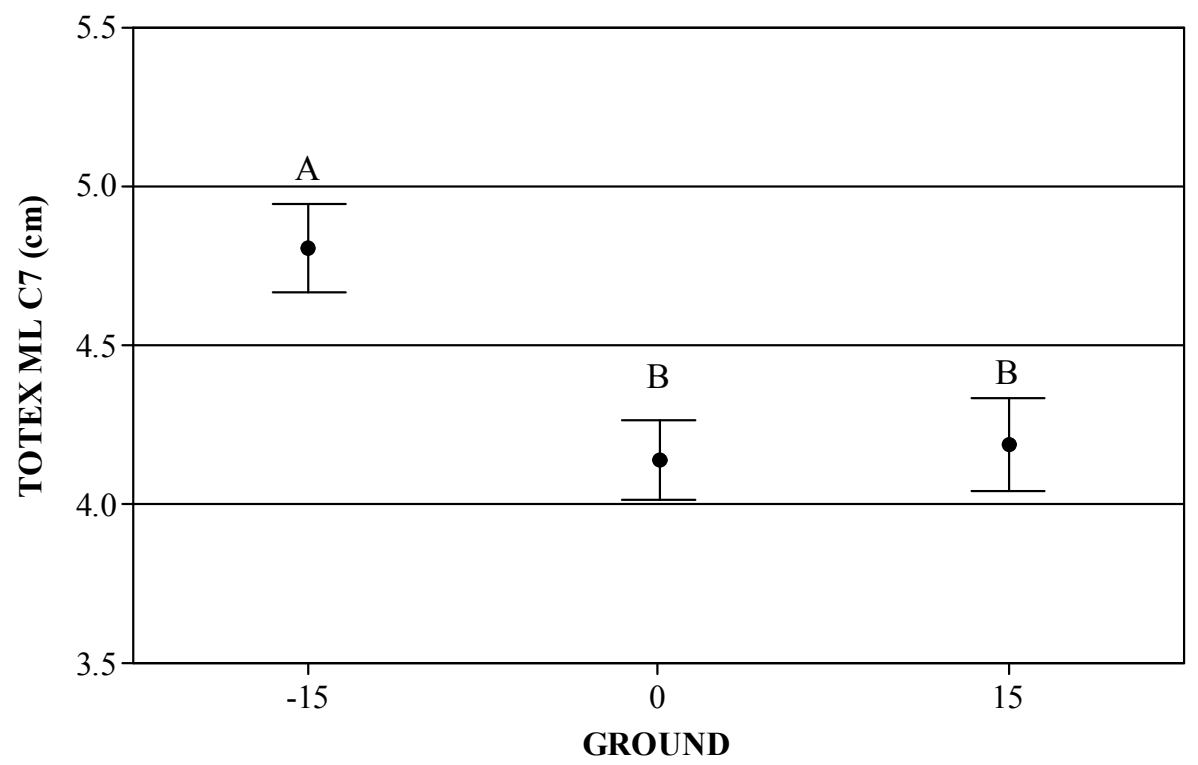

Figure 47: Effects of GROUND on C7 TOTEX ML

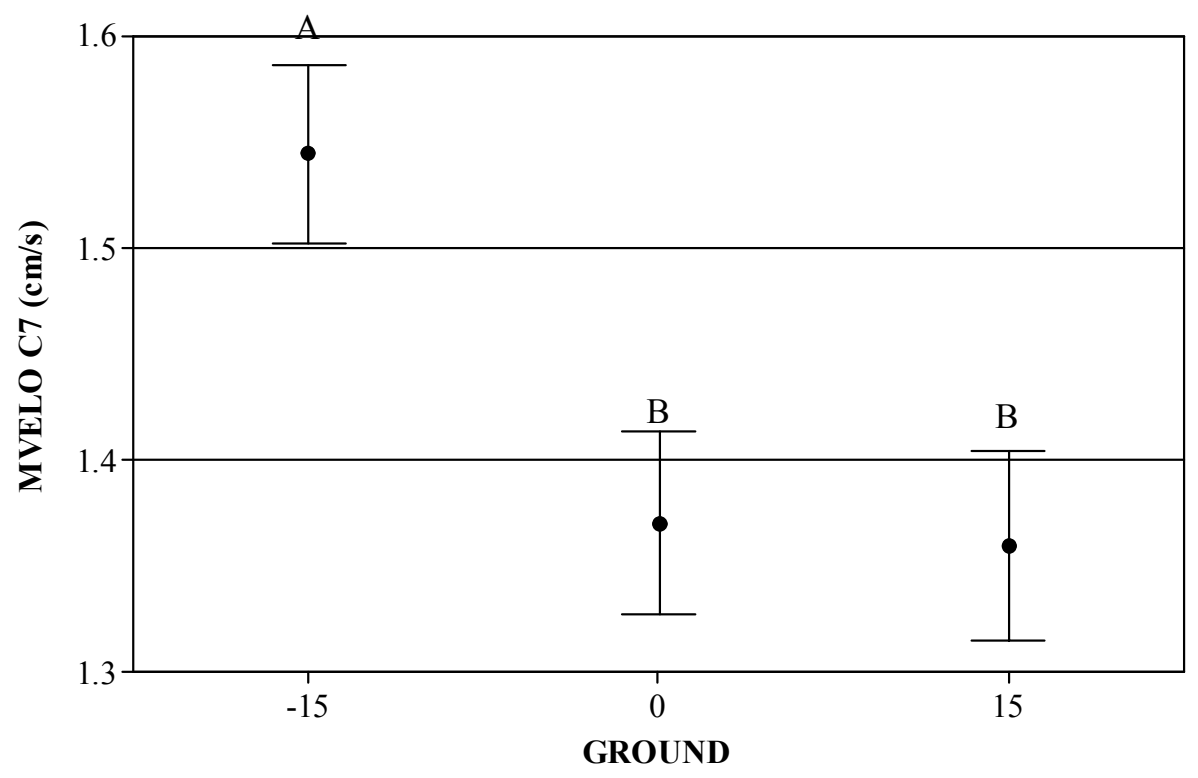

Figure 48: Effects of GROUND on C7 MVELO 


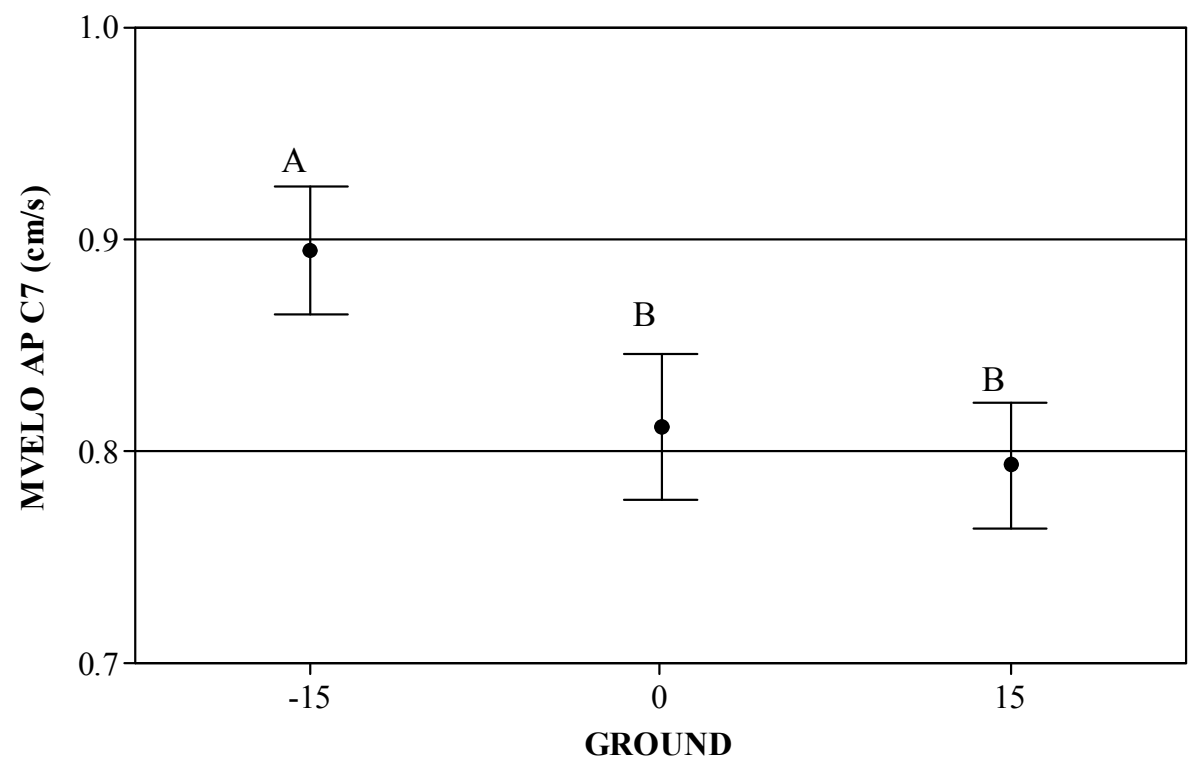

Figure 49: Effects of GROUND on C7 MVELO AP

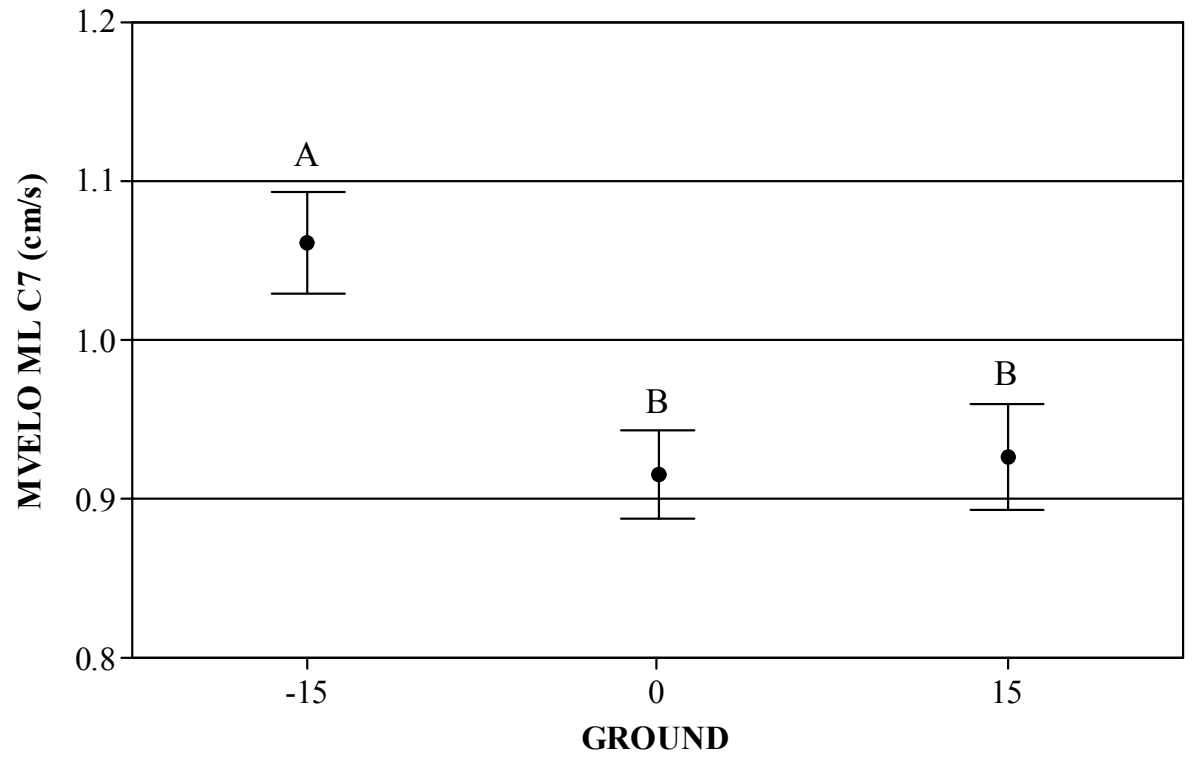

Figure 50: Effects of GROUND on C7 MVELO ML 


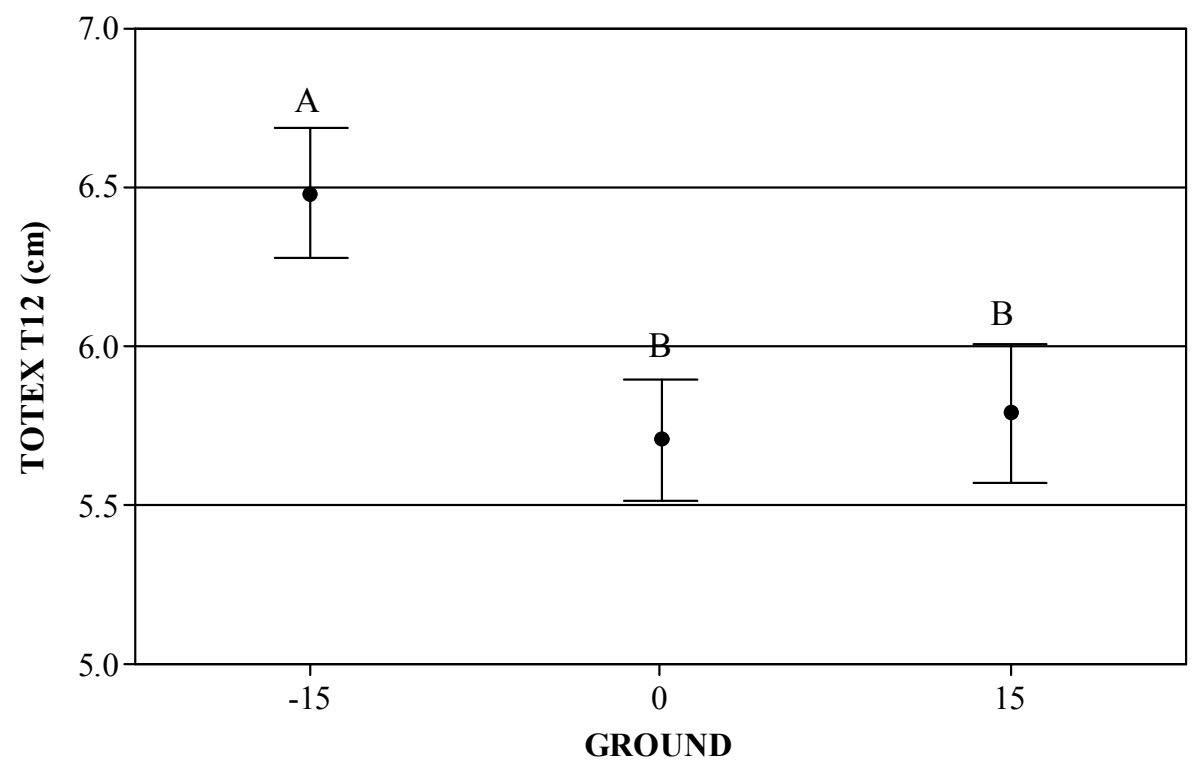

Figure 51: Effects of GROUND on T12 TOTEX

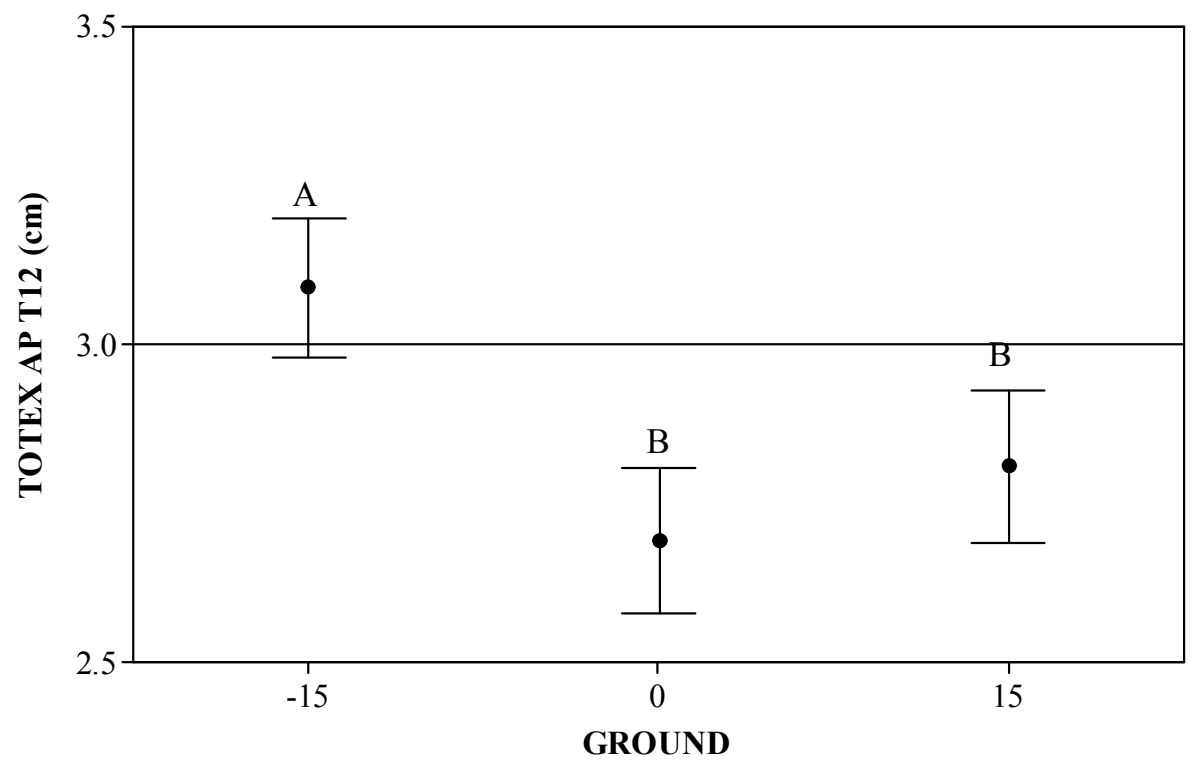

Figure 52: Effects of GROUND on T12 TOTEX AP 


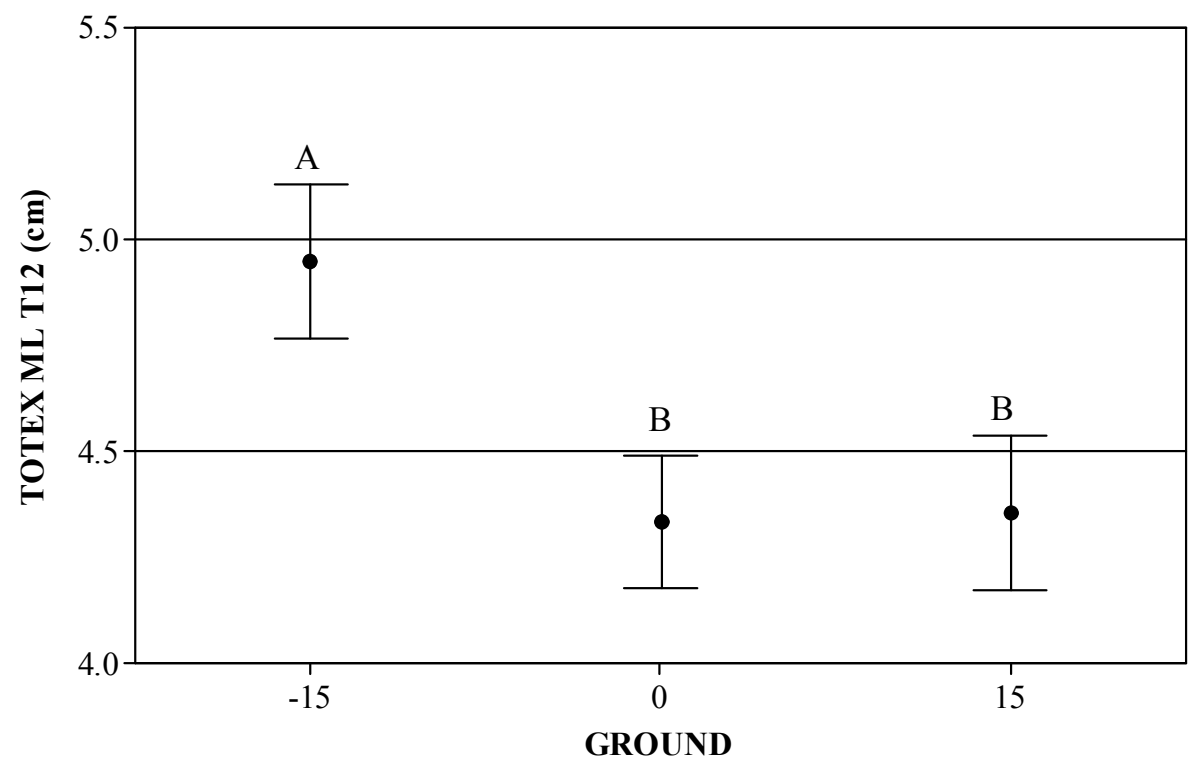

Figure 53: Effects of GROUND on T12 TOTEX ML

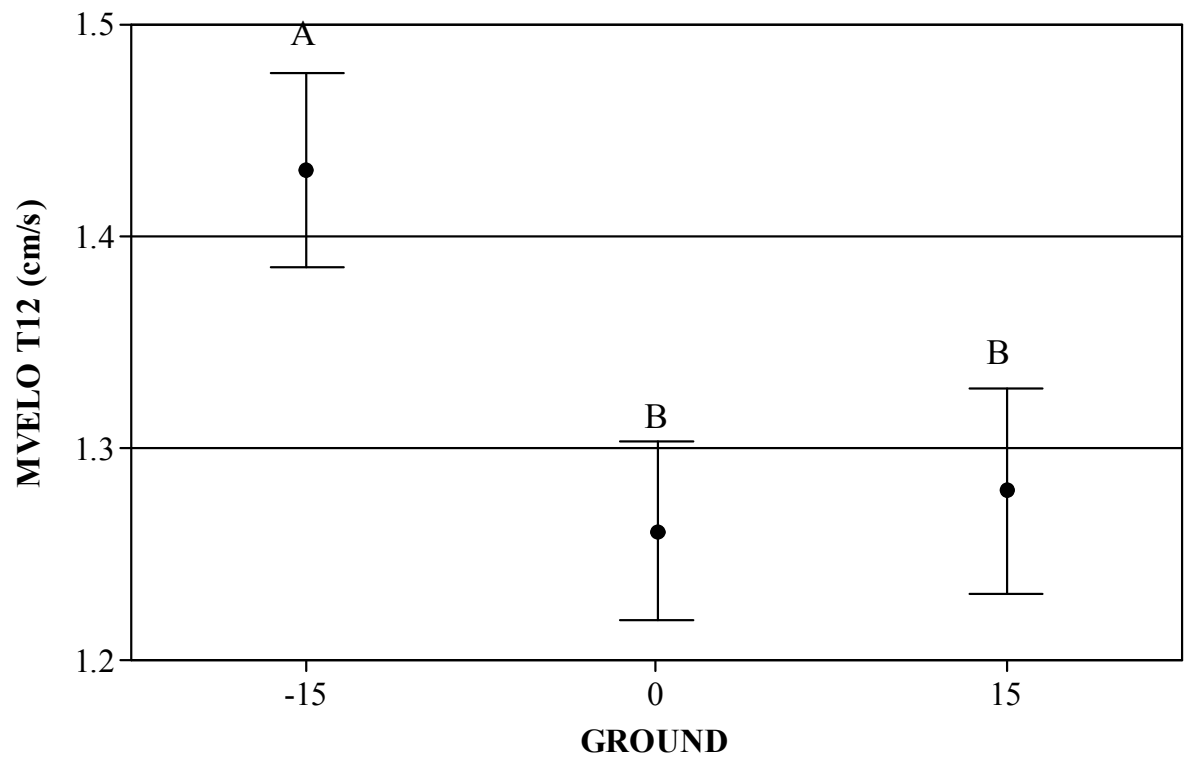

Figure 54: Effects of GROUND on T12 MVELO 


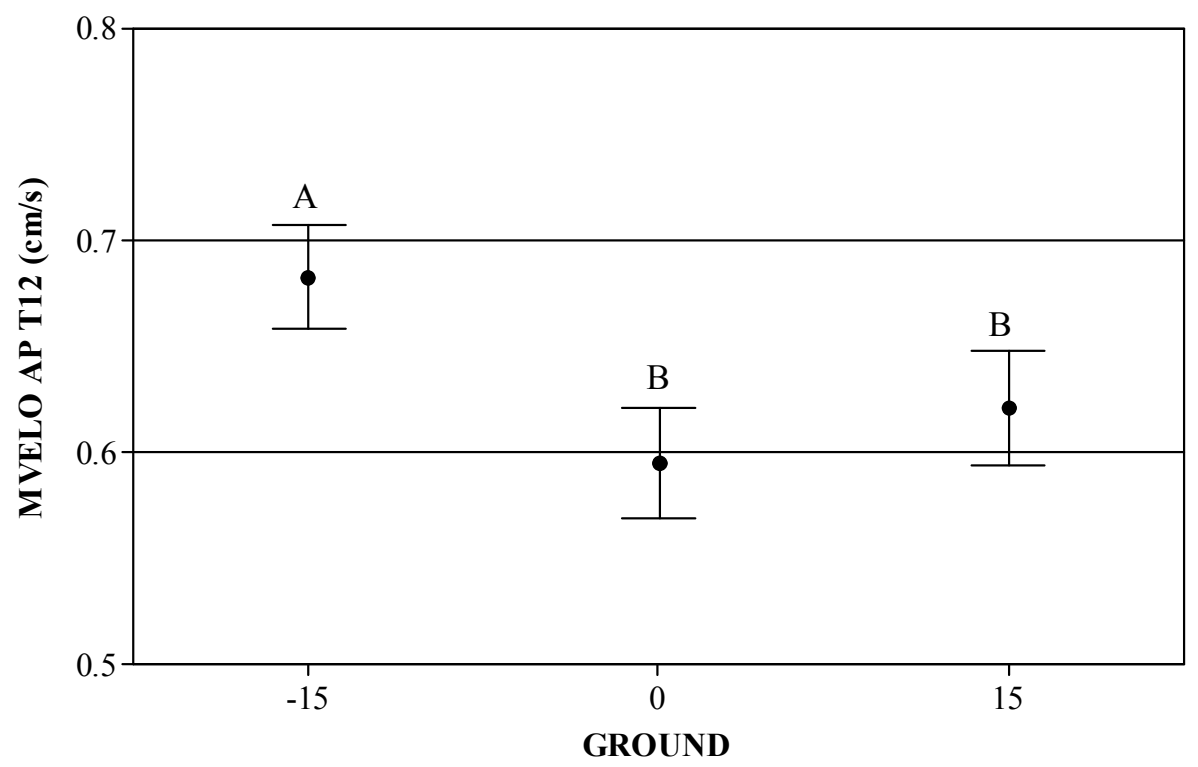

Figure 55: Effects of GROUND on T12 MVELO AP

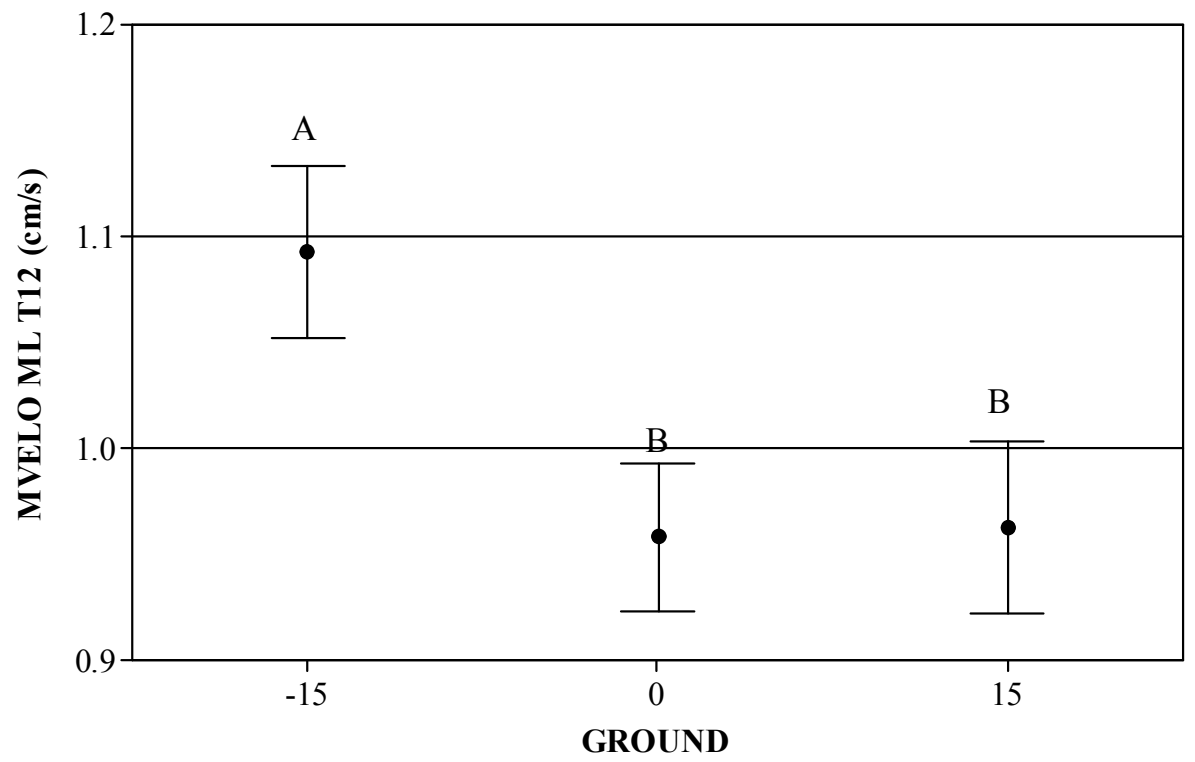

Figure 56: Effects of GROUND on T12 MVELO ML 


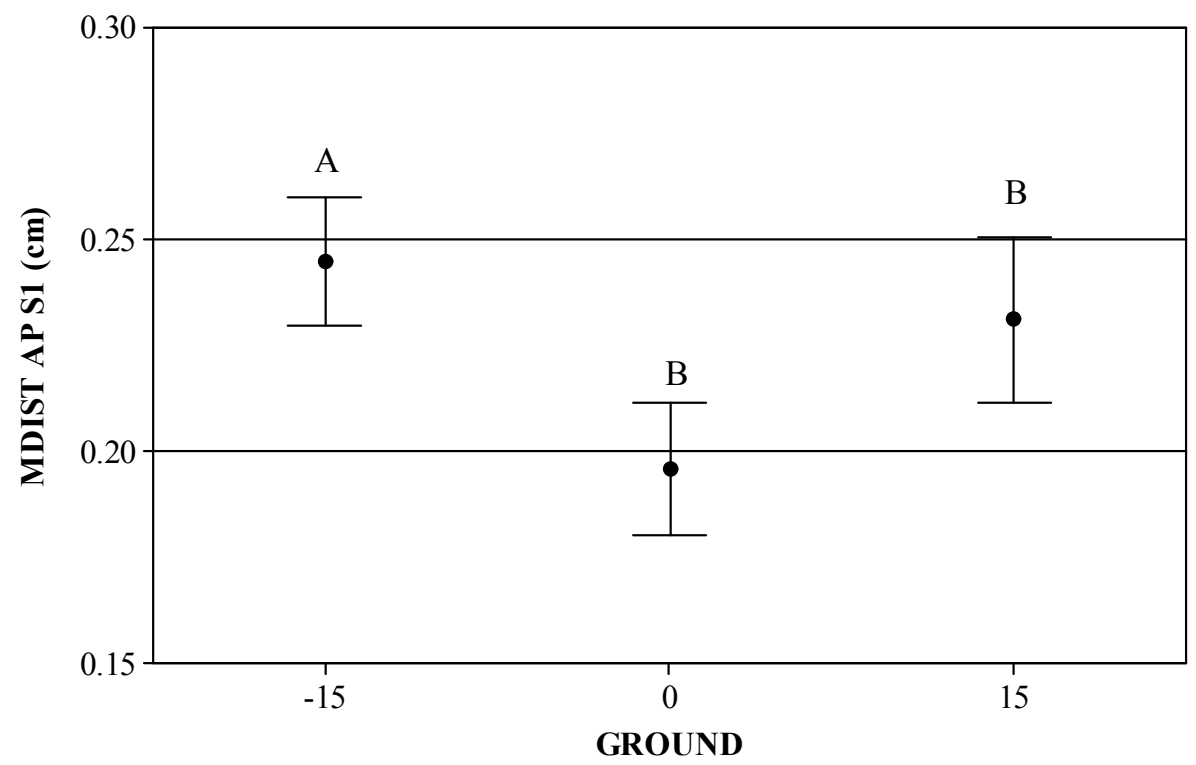

Figure 57: Effects of GROUND on S1 MDIST AP

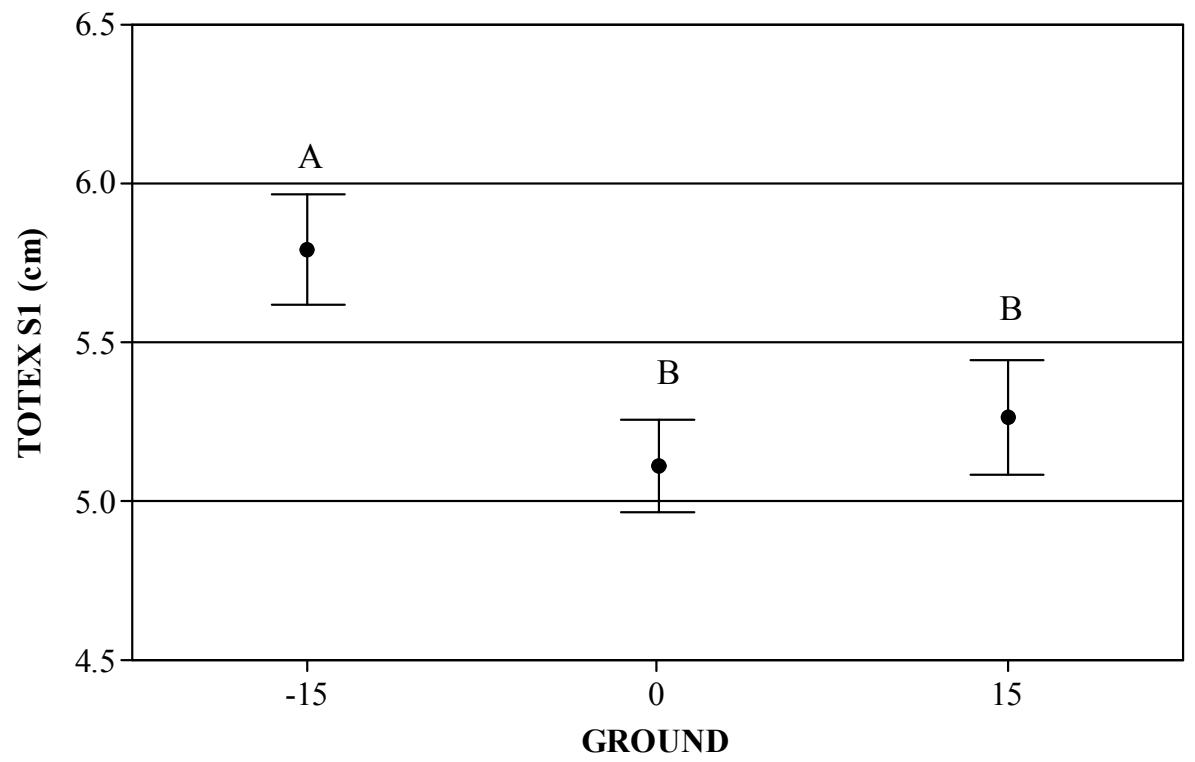

Figure 58: Effects of GROUND on S1 TOTEX 


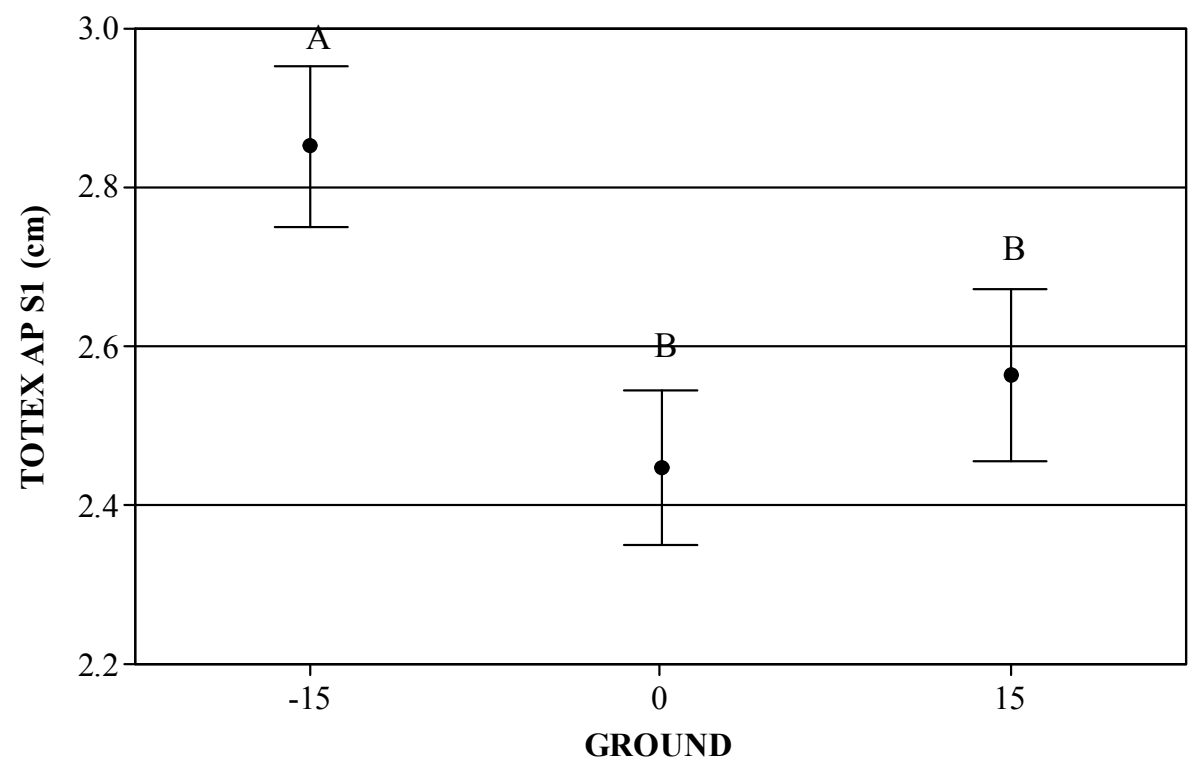

Figure 59: Effects of GROUND on S1 TOTEX AP

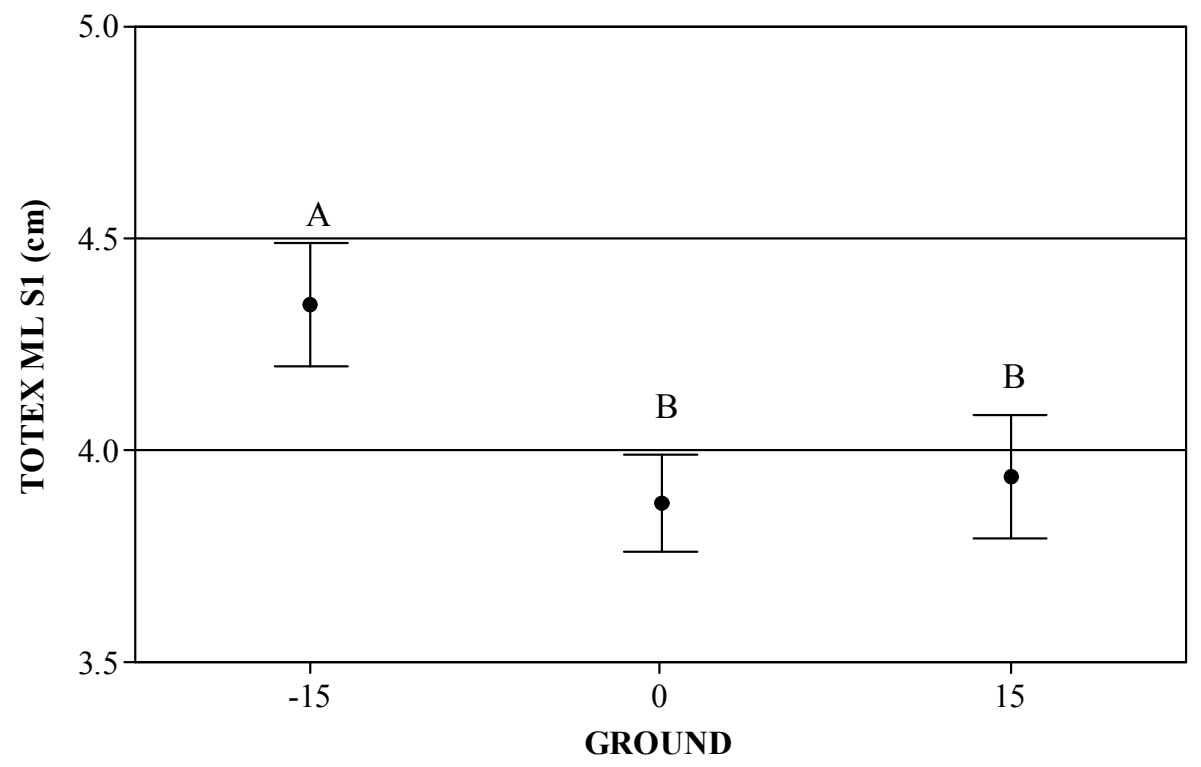

Figure 60: Effects of GROUND on S1 TOTEX ML 


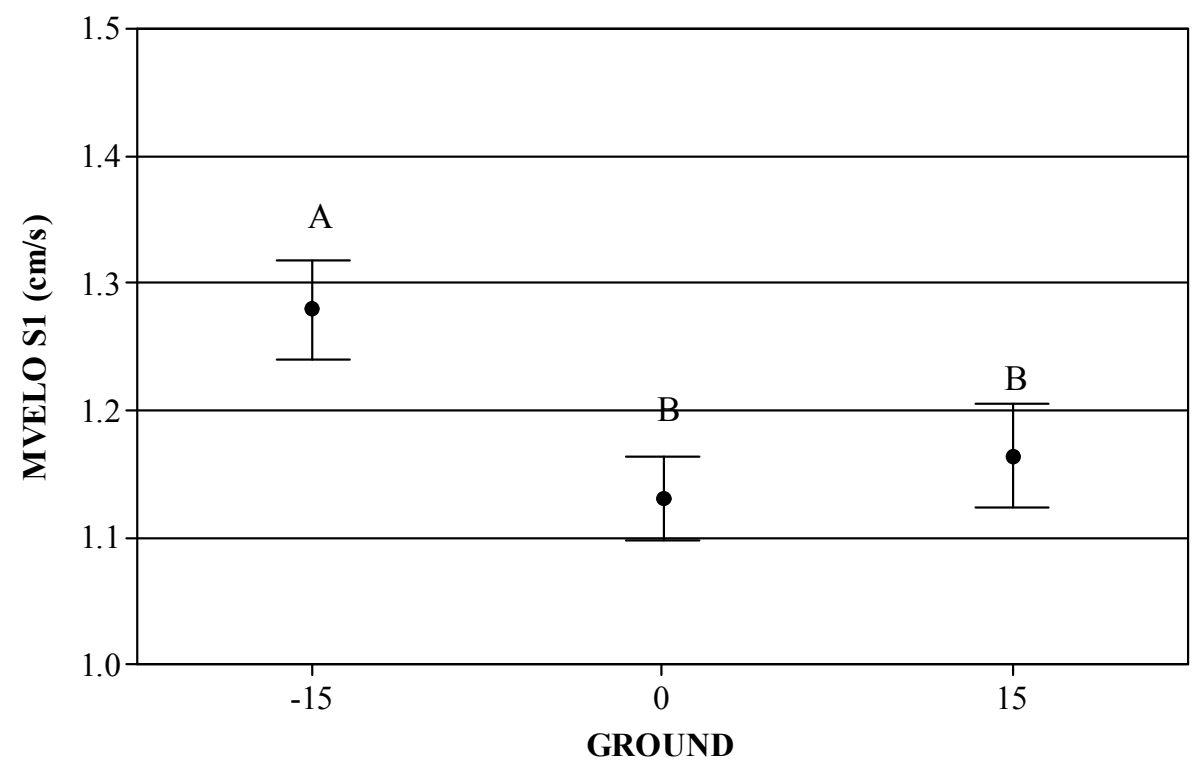

Figure 61: Effects of GROUND on S1 MVELO

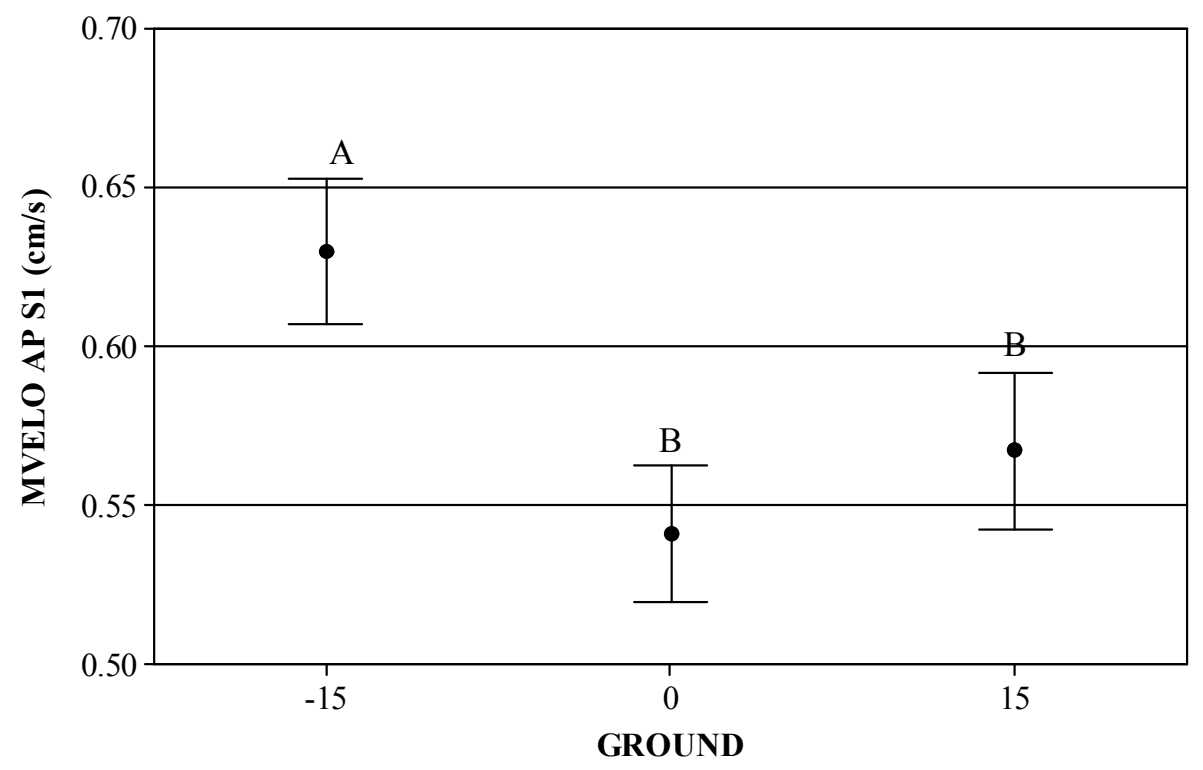

Figure 62: Effects of GROUND on S1 MVELO AP 


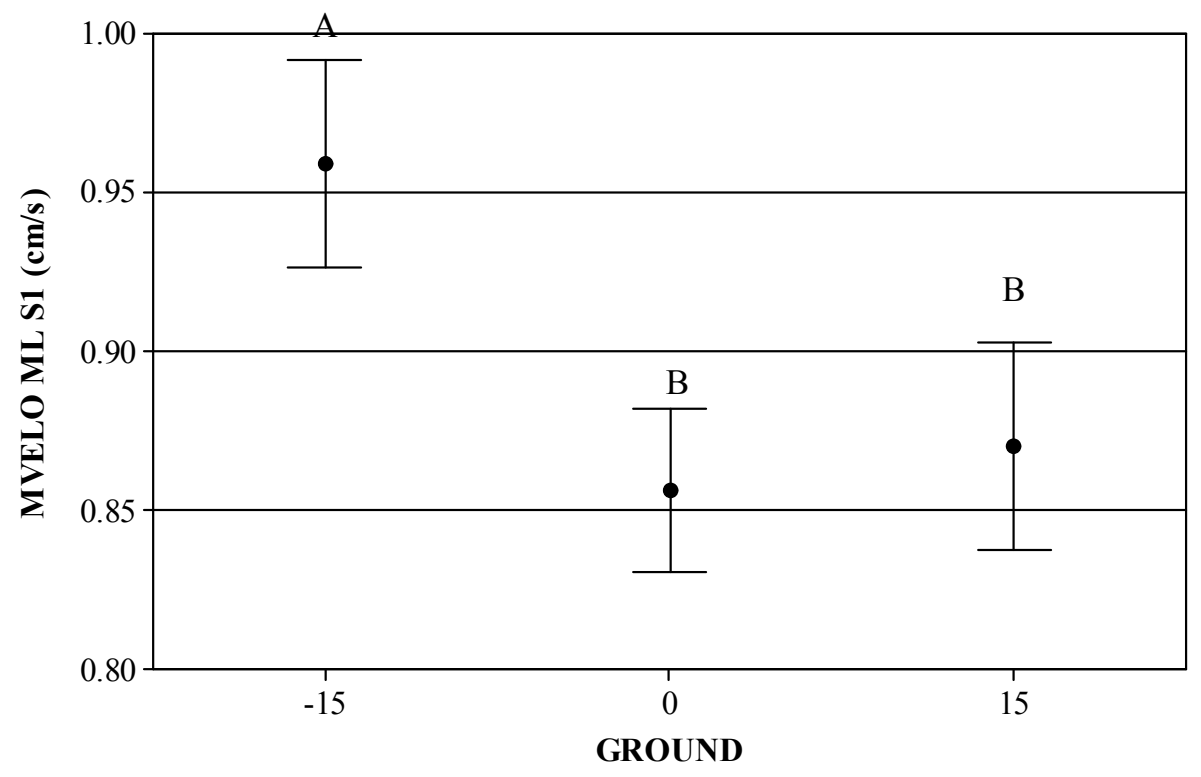

Figure 63: Effects of GROUND on S1 MVELO ML

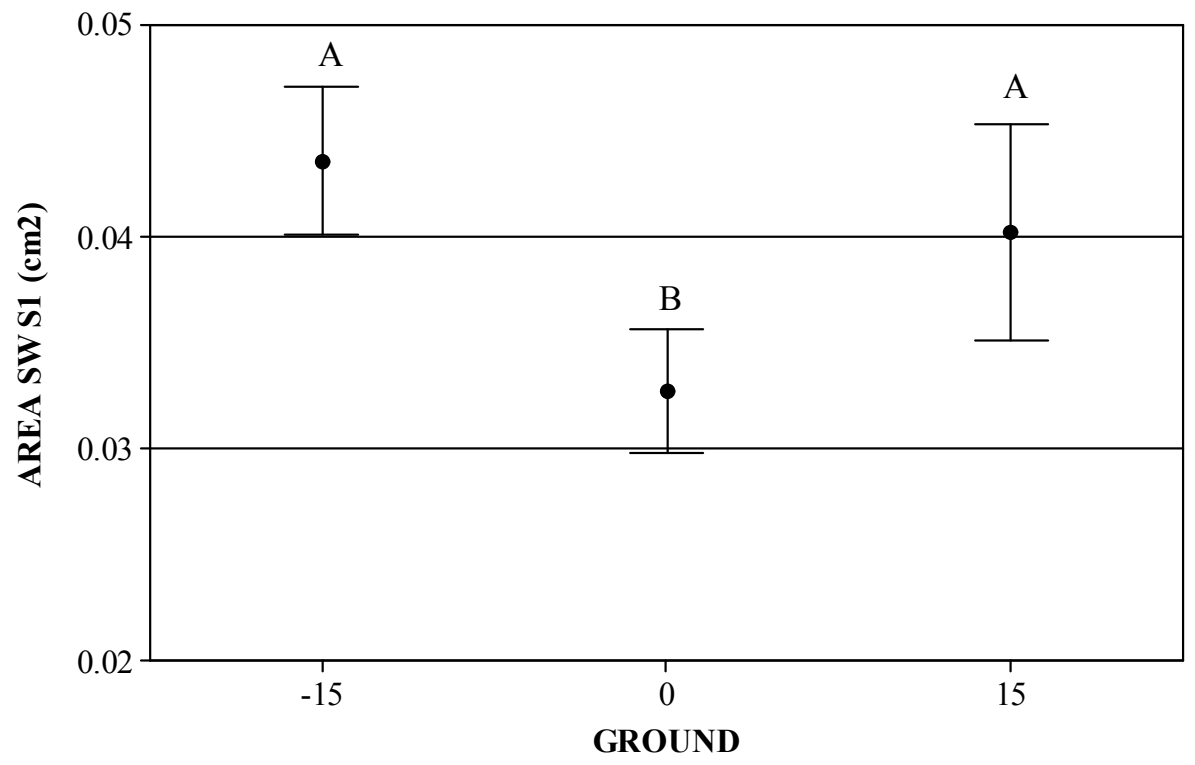

Figure 64: Effects of GROUND on S1 AREA SW 


\subsection{Discussion}

\subsubsection{Achievements}

The purpose of the current study was to investigate the effects of anterior-posteriorly slanted ground surface, trunk asymmetry and their interaction on spine biomechanical responses during the performance of trunk bending motions. Specifically, we measured the changes of lumbar extensor muscle FRP during trunk flexion-extension tasks. Based on previous literature, it was hypothesized that an uphill facing direction would reduce the EMG-off angles of lumbar extensor muscles, while a downhill facing direction would increase the onset angles. We also expected to observe reduced FRP onset angles on the contralateral side of lumbar extensor muscles during asymmetric bending. Results of the study supported our initial hypotheses and key finding has been published on Ergonomics (Hu et al., 2016). Regarding the lumbar segmental stability performance, we expected to observe that asymmetric condition and uphill facing direction could induce negative influences on participants' lumbar segmental stability performance. Analysis results supported our initial hypotheses.

Compared to the flat ground condition, performing trunk flexion on a $-15^{\circ}$ slanted surface resulted in an earlier onset of lumbar extensor muscle FRP and it was demonstrated by the reduction of lumbar and trunk EMG-off angles. Meanwhile, the $15^{\circ}$ slanted surface condition resulted in a delayed onset of lumbar extensor muscle FRP and it was demonstrated by the increase of lumbar and trunk EMG-off angles. When participants stand on the $-15^{\circ}$ slanted ground, the dorsiflexion on the ankle joints creates extra tension among Achilles tendons, the connected calf muscles and the hamstring muscles. A previous study showed that tension on the lower extremity (hamstring muscles, tendons, etc.) could affect the position of the pelvis 
(Dewberry et al., 2003). In the current study, tension on the hamstring muscles created by the ankle dorsiflexion could reduce magnitude of the pelvic rotation. The overall reduction in pelvis rotation posture may further lead to extra tension among lumbar passive tissues and generate an earlier onset of lumbar muscle FRP during trunk flexion. This argument is also supported by the significantly smaller maximum trunk inclination angle observed in the $-15^{\circ}$ condition. Through a similar mechanism, when standing on a $15^{\circ}$ slanted surface the plantar-flexion on the ankle joints reduces the tension on the Achilles tendon and the hamstring muscles, increases the pelvis rotation which induces smaller lumbar passive tissue loading. As a result, a delayed lumbar muscle FRP was observed.

From the trunk stability point of view, previous studies have discovered increased postural instability while standing on a downhill facing slanted surface (Lin \& Nussbaum, 2012). In the current study, when participants perform trunk full flexion motion while standing on the $-15^{\circ}$ slanted surface, the reduced trunk stability could trigger higher magnitude of trunk muscle cocontraction therefore delay the onset of lumbar muscle FRP. Such protective neuromuscular pattern has been previously identified as an important approach to maintain body balance (Granata et al., 2008). Next phase of data analysis will focus on stability related methods and will explore the influence of the independent variables on spinal stability in greater detail.

ASYM analysis indicated that when performing asymmetric flexion-extension task, FRP occurred much less frequently on the ipsilateral side of lumbar muscles and significantly smaller EMG-off lumbar flexion angle and trunk inclination angle were observed among the contralateral side of lumbar extensor muscles. These findings are in agreement with previous literature (Ning et al., 2011). During asymmetric bending, the flexion exertion of the lumbar 
spine is combined with multi-dimensional motions: anterior flexion, lateral bending and axial rotation are all involved. Specifically, trunk flexion using asymmetric postures induces angular lateral flexion motion which generates larger passive tissue tension on the other side and this tension is in direct proportion to the lateral distance of these passive tissues from the center of the lumbar (i.e. magnitude of the moment arm). With a longer moment arm the passive tissues on the contralateral side are placed in tension status earlier in the flexion motion than during the normal sagittally symmetric flexion tasks. This underlying mechanism is also supported by the results of the current study: both maximum lumbar flexion angle and maximum trunk inclination angle were considerably smaller with greater asymmetry condition.

The current results also showed that the maximum trunk inclination angle was significantly affected by the interaction effect of ASYM and GROUND: under symmetric bending condition subjects showed the largest trunk inclination angle on the flat ground. While, in the asymmetric bending condition the influence of GROUND was not significant. It is possible that, when standing on the $-15^{\circ}$ slanted ground, the elevated tension on hamstring reduced the magnitude of trunk bending, while in the $15^{\circ}$ slanted ground the decreased trunk stability increased muscle cocontraction and the associated joint stiffness, thereby reduced the maximum trunk inclination angle. On the other hand, such effect of GROUND was not significant in the asymmetric bending condition, possibly due that the strong influence of asymmetry on the maximum trunk angle (note the clear decrease of maximum trunk angles in the asymmetric bending condition) may have covered the influence of GROUND.

Human postural control is achieved through input from the vestibular, visual and proprioceptive systems and is organized by the central nervous system (CNS) (Brumagne et al., 2004). 
Traditionally, postural control and body sway can be assessed by measuring the deviation in the location of the center of pressure (COP) on the supporting surface by the means of a force platform. Over the past 20 years, the center of pressure (COP) has been used as an index of postural control in standing posture (Pline et al., 2006; Simeonov et al., 2009; Lin and Nussbaum 2012). However, COP is an indicator of the overall body stability, while local segmental stabilities are not able to be fully illustrated using COP. In the current study, we investigated spinal stability at three different motion segment levels, compare to COP, these motion based measurement are able to provide a clear view of how spinal stability was affected at different levels. To the best knowledge of author, no such investigation has been conducted before, and the exact effect of slanted ground surfaces and motion asymmetry on participants' lumbar segmental stability remains unclear.

Results of the current research demonstrated that ASYM significantly affected participants' lumbar segmental stability performance at all three segments (C7, T12 and S1). In asymmetric conditions, participants showed larger sway distance, sway velocity and sway area (Table 12 to Table 14). Specifically, in the asymmetric condition: C7 segment MDIST increased from 0.61 to $0.75 \mathrm{~cm}$ (p-value $<0.001)$ and RDIST increased from 14.67 to $17.86 \mathrm{~cm}$ (p-value $<0.001)$. Its AREA CC also increased significantly from 24.89 to $39.76 \mathrm{~cm}^{2}$ (p-value $\left.=0.001\right)$, AREA CE increased from 20.5 to $33.81 \mathrm{~cm}^{2}$ (p-value $<0.001$ ) and AREA SW increased from 0.061 to $0.075 \mathrm{~cm}^{2} /$ unit of time ( $\mathrm{p}$-value $\left.=0.012\right)$. However, sway velocity (i.e. MVELO) was not affected by ASYM significantly. In general, T12 and S1 segments showed a similar trend. A major difference between the symmetrical and asymmetrical posture was the non-typical weight distribution between the two legs. Although we did not measure the actual body weight distribution in the current research, it seems reasonable to assume that the asymmetric task 
induced the left leg to act as the supporting leg, bearing more body weight. The alternation of weight distribution may affect the performance of human proprioceptive systems and reduce the lumbar segmental stability.

Results also indicated that ASYM had more substantial effects on the AP direction compared with the ML direction. The current research required participants to maintain a deep sagittal bending posture which is more challenging for the proprioceptive acuity of the lumbar spine in the AP direction, due to drastic posture changes compared with the upright standing posture. One study has confirmed that impaired proprioception could delay stabilizing muscle activation and lead to decrements in postural control (Davidson et al., 2004). Although the CNS may reweight sensory inputs to achieve effective control of balance (Oie et al., 2002; Horak \& Macpherson, 1996), the overall correction effects in the AP direction may not be as good as in the ML direction which was demonstrated in the current study.

In addition, analyses results demonstrated that AREA CE is more robust and appropriate to be used in investigating the influence of ASYM on lumbar segmental stability, as the other two sway area variables (AREA CC and AREA SW) failed to detect significant changes between different conditions. One mechanism may be responsible for the differential effect: the influence of ASYM on lumbar segmental stability in the ML direction was much less noticeable than in the AP direction. Moreover, the algorithm of AREA CC calculation is not capable of addressing the unbalanced changes in the two axes. In other words, the algorithm would obtain a circle with a radius that covers $95 \%$ of the sway profile; and, due to the unbalanced changes in the two axes, this circle would include more empty space. Therefore, AREA CC could possibly fail to detect changes unless ASYM had major effects on both axes. On the other hand, the AREA CE has two 
radii that need to be calculated, and thus is more capable of addressing this issue. In the current study, changes in the AP direction would be quantified by the length of one radii (either major radii or minor radii, depends on the definition of the ellipse) while changes in the ML direction would be quantified by the length of another radii. As a result, AREA CE would be able to detect changes even if ASYM only had significant effect in one axis.

Our results indicated that uphill facing ground condition generated the most unstable lumbar segmental stability performance. More specifically, C7 segment showed much larger sway velocity (AP, ML and overall) and total excursion when participants stepped on the uphill facing slanted surface. However, even though the magnitude of MDIST and RDIST were physically larger in uphill facing condition, such differences failed to achieve any statistical significance, potentially due to the mild slanted surfaces used in the current research. T12 and S1 segments showed similar patterns in general. This result is in agreement with previous literature (Mezzarane \& Kohn, 2007; Lin \& Nassbaum, 2012). One study found that the uphill facing direction generated a higher instability, which was reflected in the higher spectral amplitudes of $\mathrm{COP}$ at lower frequencies (i.e. $<0.3 \mathrm{~Hz}$ ). However, in that study authors also concluded that the downhill facing direction had lower spectral amplitudes of COP at lower frequency than the flat ground condition, suggesting that the downhill facing direction was more desirable. Here, uphill inclination induced postural instability, while the positive effects of the downhill facing direction were not observed. This discrepancy related to downhill facing direction may be attributed to the different experimental designs between studies. In the current research, participants were required to maintain a deep flexion posture which forced their COP to move forward and induced a higher tendency of falling. This change would generate negative effects on the lumbar segmental stability and may counterbalance the influences of lower spectral amplitudes. 
Several mechanisms may be responsible for the differential effects found between the uphill and downhill facing directions. Uneven surfaces could be either lengthening or shortening the ankle plantar flexors, and this alternation could change ankle muscle activity level. One study demonstrated that soleus activity increased in the downhill facing direction but was unchanged in the uphill facing direction (Mezzarane \& Kohn, 2007). Another study reported increased soleus activity in the downhill facing direction but decreased activity in the uphill facing direction (Sasagawa et al., 2009). The ability to avoid undesirable responses triggered by internal or external perturbations is known to be an important property of the human postural control system (Gurfinkel et al., 1995). The changes of muscle activation pattern could potentially alter the ability to avoid the perturbation and decrease the lumbar segmental stability. However, further work is needed to confirm the above mechanism. Another explanation came from the discomfort inhibition point of view. Previous literature has reported that dysfunction in trunk muscle control (Hodeges and Richardson 1996; Hides et al., 1996) could affect human stability performance. In the current research when participants stepped on the uphill facing surface, the dorsiflexion on the ankle joints creates extra tension among Achilles tendons, the connected calf muscles, and other lower extremity muscles. Discomfort in these regions could cause an increased presynaptic inhibition of muscle afferents (Sibley et al., 2007) and prolonged latencies and decreased muscle control and increased postural sway (Capra and Ro 2000).

Another important finding from the current research was that $\mathrm{C} 7$ segment demonstrated the best stability performance, as compared to $\mathrm{S} 1$ and $\mathrm{T} 12$ segments. This result could be attributed to the fact that when a deep trunk bending posture was maintained, C7 segment became the most proximal segment among the three, while S1 became the most dismal segment relative to the ground and therefore had the worst segmental stability performance. Another possible 
explanation is that one of the core components of the human balance control system, vestibular sub-system, is located close to the C7 segment, and the CNS has higher priority to stabilize this region to achieve the optimized overall system level performance.

\subsubsection{Potential implementation and future work}

A number of ergonomic risk assessment tools have been developed which allow practitioners to evaluate the risk level of certain tasks based on a set of structured factors. The first is the Ovako Working Posture Analyzing System (OWAS) which was originally developed by a company based in Finland (Karhu et al., 1977). OWAS uses factors include the back, arms and legs posture, and the weight of the load handled. Another example is the Rapid Upper Limb Assessment (RULA) (McAtameny \& Corlett, 1993). This evaluation is based on an estimation of the main upper-body, trunk and neck joint angles. Additional scores (muscle usage and forces score) were added to obtain a final grand score which divides the task into four action levels. Rapid Entire Body Assessment (REBA) uses similar structure and is primarily used to analyze the whole body risk (Hignett \& McAtamney, 2000). In addition, the original NIOSH lifting equation was published by the National Institute for Occupational Safety and Health (NIOSH) in 1981, with a newer iteration released in 1991 (NIOSH 1981, Waters et al., 1993). The equation includes seven parameters: Horizontal Multiplier (HM), Vertical Multiplier (VM), Distance Multiplier (DM), Asymmetric Multiplier (AM), Frequency Multiplier (FM), Load Constant (LC)

and Coupling Multiplier (CM). The overall score is rendered by combining the effects of all the seven parameters. 
Above ergonomics assessment tools are generally accepted and used widely with the advantages of high cost effectiveness in field investigations and minimal disturbance to worker task performance. However, neither of the above assessment tools have taken working surfaces into consideration, nor included working surface as a modification factor. For example, the NIOSH lifting equation could add the $8^{\text {th }}$ factor and take the magnitude of the slanted ground surface into determining the recommended lifting limit. REBA and RULA already consider the posture of the lower extremity when evaluating the overall risk level. However, these methods still overlook the influence of the working surface since workers could still maintain the same lower extremity posture and adjusting the ankle flexion or extension angles when stepping on slanted surfaces. Therefore, it would be beneficial to modify the current assessment tools and add the working surface parameter to the next iteration of those tools. Results from the current research could serve as the first step of quantifying the design of those parameters.

The current study confirmed uneven ground surfaces could significantly affect human spine biomechanics and segmental stability. In the future, follow up experiments will be required to obtain the functions that describe all of the relationships between ground surfaces and human spine biomechanics. One possible way of obtaining this information is to design an experiment with more levels of slanted surfaces. The current research only considered slanted surfaces in three levels. From the results, it is difficult to verify whether the relationship between uneven ground surfaces and segmental stability is linear. 


\subsubsection{Limitations}

Several limitations restrict the generalizability of this study. First, due to safety considerations to reduce the risk of falling, only moderate slanted surfaces (i.e. $+/-15^{\circ}$ ) were tested. Steeper slanted ground surfaces may result in the absence of lumbar muscle FRP due to further reductions in trunk stability and the possibly elevated trunk co-contraction.

Second, only male participants were recruited for the current data collection. Previous literature has shown significant tissue property differences between males and females. This difference may affect general spinal biomechanical responses. Further investigation is needed to analyze the influence of gender on spinal biomechanical responses when standing on the antero-posteriorly slanted ground surfaces.

Third, all participants were young college students. This sample group was overall lacking long term real world occupational experience of working on uneven ground surfaces. Therefore, their biomechanical responses (kinematics, foot-weight distributions, muscle activation patterns, etc.) could be different from workers who have multiple year experiences of working on laterally slanted surfaces.

Fourth, the neuro component responses of the stability control system due to the changes of working surfaces were not investigated in the current research. Follow up experiment may collect these data accompanied with the kinematic stability data in order to investigate the coordination between different components. 


\section{CHAPTER 5. CONCLUSION}

Our results showed that standing on a downhill facing slanted surface could delay the onset of lumbar muscle flexion relaxation phenomenon, while standing on an uphill facing ground would cause lumbar muscle flexion relaxation to occur earlier. Compared to symmetric bending, when performing asymmetric bending, flexion relaxation occurred earlier on the contralateral side of lumbar muscles and significantly smaller maximum lumbar flexion and trunk inclination angles were observed. In addition, when performing trunk flexion-extension motions on laterally slanted ground surfaces, the ipsilateral lumbar muscles will cease activity earlier than the contralateral side. The increase in laterally slanted ground angle enlarges this difference and reduces the maximum trunk flexion angle. Furthermore, laterally located lumbar muscles tend to have larger bilateral EMG-Off angular differences compared with medially located lumbar muscles.

In terms of segmental stability performance, our results showed that asymmetric posture significantly increased C7, T12 and S1 segments' sway distance, sway velocity and sway area. Uphill facing surface induced negative influences on lumbar segmental stability and is the least desirable working condition. In addition, when conducting tasks on slanted surfaces with a deep bending posture, $\mathrm{S} 1$ segment reported the worst segmental stability, compared to $\mathrm{C} 7$ and $\mathrm{T} 12$ segments.

In conclusion, our results confirmed that the uneven ground surfaces significantly influence trunk biomechanical performance and stability; these changes may elevate the risk of LBP. Results from the current research could be used to improve ergonomic assessment tools. 


\section{REFERENCES}

Alschuler, K.N., Neblett, R., Wiggert, E., Haig, A.J., Geisser, M.E., 2009. Flexion relaxation and clinical features associated with chronic low back pain: a comparison of different methods of quantifying flexion relaxation. The Clinical Journal of Pain 25: 760-766.

Andres, R.O., Holt, K.G., Kubo, M., 2005. Impact of railroad ballast type on frontal plane ankle kinematics during walking. Applied Ergonomics 36: 529-534.

Bazrgari, B., Hendershot, B., Muslim, K., Toosizadeh, N., Nussbaum, M.A., Madigan, M.L., 2011. Disturbance and recovery of trunk mechanical and neuromuscular behaviours following prolonged trunk flexion: influences of duration and external load on creep-induced effects.

Battie, M.C., Haynor, D.R., Fisher, L.D., Gill, K., Gibboons, L.E., Videman, T., 1995. Similarities in degenerative findings on magnetic resonance images of the lumbar spines of identical twins. The Journal of Bone \& Joint Surgery 77: 1662-1670.

Bergmark, A., 1989. Stability of the lumbar spine: a study in mechanical engineering. Acta Orthopaedica Scandinavica 230: 2301-2354.

BLS, 1999. National census of fatal occupational injuries. US Department of Labor, Bureau of Labor Statistics, Washington, DC.

BLS, 2007a. Census of fatal occupational injuries charts, 1992-2006. US Department of Labor, Bureau of Labor Statistics, Washington, DC.

BLS, 2007b. Number of nonfatal occupational injuries and illnesses involving days away from work by event or exposure leading to injury or illness and industry sector, 2006. US Department of Labor, Bureau of Labor Statistics, Washington, DC.

Bogduk, N., 1980. A reappraisal of the anatomy of the human lumbar erector spinae. Journal of Anatomy 131: 525-540. 
Bogduk, N., Johnson, G., Spalding, D., 1998. The morphology and biomechanics of latissimus dorsi. Clinical Biomechanics 13: 377-385.

Bogduk, N., Macintosh, J.E., Pearcy, M.J., 1991. A universal model of the lumbar back muscles in the upright position. Spine 17: 897-913.

Bongers, P.M., De Winter, C.R., Kompier, M.A.J., Hildebrandt, V.H., 1993. Psychosocial factors at work and musculoskeletal disease. Scandinavian Journal of Work 19: 297-312.

Boschman, J.S., van der Molen, H.F., Sluiter, J.K., Frings-Dresen, H.W.F., 2011. Occupational demands and health effects for bricklayers and construction supervisors: a systematic review. American Journal of Industrial Medicine 54: 55-77.

Carpa, N.F., Ro, J.Y., 2000. Experimental muscle pain produces central modulation of proprioceptive signals arising from jaw muscle spindles. Pain 86: 151-162.

Cassidy, J.D., Carroll, L.J., Cote, P., 1998. The Saskatchewan health and back pain survey. The prevalence of low back pain and related disability in Saskatchewan adults. Spine 23: 1860-1867.

Cecchi, F., Debolini, P., Lova, R.M., Macchi, C., Bandinellli, S., Bartali, B., Lauretani, F., Benvenuti, E., Hicks, G., Ferrucci, L., 2006. Epidemiology of back pain in a representative cohort of Italian persons 65 years of age and older: The InCHIANTI study. Spine 31: 1149-1155.

Chaffin, D.B., Andersson, G.B.J., Martin, B.J., 2006. Occupational Biomehcanics (4 ${ }^{\text {th }}$ ed.). New York: Wiley-Interscience.

Cholewicki, J., Juluru, K., McGill, S.M., 1999. Intra-abdominal pressure mechanism for stabilizing the lumbar spine. Journal of Biomechanics 32: 13-17.

Coenen, P., Kingma, I., Boot, C.R., 2012. The contribution of load magnitude and number of load cycles to cumulative low back load estimations: a study based on in-vitro compression data. Clinical Biomechanics 27: 1083-1086. 
Cresswell, A.G., Grundstrom, H., Thorstensson, A., 1992. Observations on intra-abdominal pressure and patterns of abdominal intra-muscular activity in man. Acta Physiologica Scandinavica 144: 409-418.

Davidson, B.S., Madigan, M,I., Nussbaum, M.A., 2004. Effects of lumbar extensor fatigue and fatigue rate on postural sway. European Journal of Applied Physiology 93: 183-189.

De Beeck, R.O., Hermans, V., 2000. Work-related low back disorders. European Agency for Safety and Health at Work.

Dewberry, M.J., Bohannon, R.W., Tiberio, D., Murray, R., Zannotti, C.M., 2003. Pelvic and femoral contributions to bilateral hip flexion by subjects suspended from a bar. Clinical Biomechanics 18: 494-499.

Deyo, R.A., Mirza, S.K., Martin, B.I., 2006. Back pain prevalence and visit rates. Estimates from U.S. national surveys, 2002. Spine 31: 2724-2727.

Dickey, J.P., McNorton, S., Potvin, J.R., 2003. Repeated spinal flexion modulates the flexion relaxation phenomenon. Clinical Biomechanics 18: 783-789.

Dionne, C., Dunn, K.M., Croft, P.R., 2006. Does back pain prevalence really decrease with increasing age? A systematic review. Age and Ageing 35: 229-234.

Dolan, P., Mannion, A.F., Adams, M.A., 1994. Passive tissues help the back muscles to generate extensor moments during lifting. Journal of Biomechanics 27: 1077-1085.

Dorland, W.A.N., 2011. Dorland's illustrated medical dictionary (32th ed.). Amsterdam, Netherlands: Elsevier and Saunders.

Dyhre-Poulsen, P., Krogsgaard, M.R., 2000. Muscular reflexes elicited by electrical stimulation of the anterior cruciate ligament in humans. Journal of Applied Physiology 89: 2191-2195. 
Fathallah, F.A., 2010. Musculoskeletal disorders in labor-intensive agriculture. Applied Ergonomics 41: 738-743.

Floyd, W.F., Silver, P.H.S., 1955. The function of erectores spinae muscles in certain movements and postures in man. Journal of Physiology 129: 184-203.

Freburger, J.K., Holmes, G.M., Agans, R.P., Jackman, A.M., Darter, J.D., Wallace, A.S., Castel, L.D., Kalsbeek, W.D., Carey, T.S., 2009. The rising prevalence of chronic low back pain. JAMA Internal Medicine 169: 251-258.

Gallagher, S., 2008. Reducing low back pain and disability in mining. Department of Health and Human Services. Centers for Disease Control and Prevention, National Institute for Occupational Safety and Health (NIOSH), Pittsburgh Research Laboratory.

Gallagher, K.M., Wong, A., Callaghan, J.P., 2012. Possible mechanisms for the reduction of low back pain associated with standing on a sloped surface. Gait \& Posture 37: 313-318.

Gardner-Morse, M., Stokes, I.A.F., Laible, J.P., 1995. Role of muscles in lumbar stability in maximum extension efforts. Journal of Orthopaedic Research 13: 802-808.

Gaskin, D.J., Richard, P., 2012. The economic costs of pain in the United States. The Journal of Pain 13: 715-724.

Gillen, M., Faucett, J.A., Beaumont, J.J., McLoughlin, E., 1997. Injury severity associated with nonfatal construction falls. American Journal of Industrial Medicine 32: 647-655.

Gollhoffer, A., Komi, P.V., Miyashita, M., Aura, O., 1987. Fatigue during stretch-shortening cycle exercises: changes in mechanical performance of human skeletal muscle. International Journal of Sports Medicine 8: 71-78. 
Gore, M., Sadosky, A., Stacey, B.R., Tai, K., Leslie, D., 2012. The burden of chronic low back pain: clinical comorbidities, treatment patterns, and health care costs in usual care settings. Spine 37: e668-e677.i

Gurfinkel, V.S., Ivanenko, Y., Levik, Y., Babakova, I.A., 1995. Kinesthetic reference for human orthograde posture. Neuroscience 68: 229-243.

Granata, K.P., Marras, W.S., 1995. The influence of trunk muscle coactivity upon dynamic spinal loads. Spine 20: 913-919.

Granata, K.P., Marras, W.S., 2000. Cost-benefit of muscle cocontraction in protecting against spinal instability. Spine 25: 1398-1404.

Granata, K.P., Orishimo, K.F., 2001. Response of trunk muscle coactivation to changes in spinal stability. Journal of Biomechanics 34: 1117-1123.

Granata, K.P., Rogers, E., Moorhouse, K., 2005. Effects of static flexion relaxation on paraspinal reflex behavior. Clinical Biomechanics 20: 16-24.

Granata, K.P., Slota, G.P., Wilson, S.E., 2004. Influence of fatigue in neuromuscular control of spinal stability. Human Factor 46: 81-91.

Gupta.A., 2001. Analyses of myoelectrical silence of erectors spinae. Journal of Biomechanics 34: 491-496.

Heuch, I., Hagen, K., Heuch, I., Nygaard, O., Zwart, J.A.., 2010. The impact of body mass index on prevalence of low back pain. Spine 35: 764-768.

Hignett, S., McAtamney, L., 2000. Rapid Entire Body Assessment: REBA. Applied Ergonomics 31: 201-205. 
Hlavacka, F., Mergner, T., Krizkova, M., 1996. Control of the body vertical by vestibular and proprioceptive inputs. Brain Research Bulletin 40: 431-435.

Hodges, P., Gandevia, S.C., 2000. Changes in intra-abdominal pressure during postural and respiratory activation of the human diaphragm. Journal of Applied Physiology 89: 967-979.

Hides, J.A., Richardson, C.A., Jull, G.A., 1996. Multifidus muscle recovery is not automatic after resolution of acute, first-episode low back pain. Spine 21: 2763-2769.

Hodges, P.W., Richardson, C.A., 1996. Inefficient muscular stabilization of the lumbar spine associated with low back pain: a motor control evaluation of transversus abodminis. Spine 21: 2640-2650.

Hoogendoorn, W.E., Bongers, P., Henrica, C.W., Douwes, M., Koes, B.W., Miedema, M.C., Geertje, A.M., Bouter, L.M., 2000. Flexion and rotation of the trunk and lifting at work are risk factors for low back pain. Spine 25: 3087-3092.

Hoy, D., Bain, C., Williams, G., March, L., Brooks, P., Blyth, F., Woolf, A., Vos, T., Buchbinder, R., 2012. A systematic review of the global prevalence of low back pain. Arthritis \& Rheumatism 64: 2028-2037.

Hu, B., Ning, X., Nimbarte, A.D., 2013. The changes of lumbar muscle flexion-relaxation response due to laterally slanted ground surfaces. Ergonomics 56: 1295-1303.

Hu, B., Ning, X., Dai, F., Almuhaidib, I., 2015. The changes of lumbar muscle flexion-relaxation phenomenon due to antero-posteriorly slanted ground surfaces. Ergonomics, DOI: 10.1080/00140139.2015.1124146.

Huang, Q.M., Thorstensson, A., 2000. Trunk muscle strength in eccentric and concentric lateral flexion. European Journal of Applied Physiology 83: 573-577. 
Hughes, R.E., Bean, J.C., Chaffin, D.B., 1995. Evaluating the effect of co-contraction in optimization models. Journal of Biomechanics 28: 875-878.

Jin, S., Ning, X., Mirka, G.A., 2012. An algorithm for defining the onset and cessation of the flexion relaxation phenomenon in the low back musculature. Journal of Electromyography and Kinesiology 22: 376-382.

Karhu, O., Kansi, P., Kuorinka, I., 1977. Correcting working postures in industry: A practical method for analysis. Applied Ergonomics 8: 199-201.

Kerr, M.S., Frank, J.W., Shannon, H.S., Norman, R.W.K., Wells, R.P., Neumann, W.P., 2001. Biomechanical and psychosocial risk factors for low back pain at work. American Journal of Public Health 91: 1069-1075.

Koch, H., Smith, M.C., 1985. Office based ambulatory care for patients 75 years old and over: national ambulatory medical care survey, 1980 and 1981. Advance data from vital and health statistics. Public Health Service 110: 1-14.

Kristiansson, P., Svardsudd, K., 1996. Back pain during pregnancy. A prospective study. Spine 21: 702-709.

Leboeuf-Yde, C., 1999. Smoking and low back pain: a systematic literature review of 41 journal articles reporting 47 epidemiologic studies. Spine 24: 1463-1470.

Leboeuf-Yde, C., 2000. Body weight and low back pain: a systematic literature review of 56 journal articles reporting on 65 epidemiologic studies. Spine 25: 226-237.

Lee, P.J., Rogers, E.L., Granata, K.P., 2006. Active trunk stiffness increases with co-contraction. Journal of Electromyography and Kinesiology 16: 51-57.

Leigh, J.P., Miller, T.R., 1997. Ranking occupations based upon the costs of job-related injuries and diseases. Journal of Occupational and Environmental Medicine 39: 1170-1182. 
Leigh, J.P., Robbins, J.A., 2004. Occupational disease and workers' compensation: coverage, costs, and consequences. The Milbank Quarterly 82: 689-721.

Leigh, J.P., 2011. Economic burden of occupational injury and illness in the United States. Milbank Quarterly 89: 728-772.

Leigh, J.P., Sheetz, R.M., 1989. Prevalence of back pain among fulltime United States workers. British Journal of Industrial Medicine 46: 651-657.

Liira, J.P., Shannon, H.S., Chambers, L.W., Haines, T.A., 1996. Long-term back problems and physical work exposures in the 1990 Ontario health survey. American Journal of Public Health 86: $382-387$.

Lin, D., Nussbaum, M.A., 2012. Effects of lumbar extensor fatigue and surface inclination on postural control during quiet stance. Applied Ergonomics 43: 1008-1015.

Luo, X., Pietrobon, R., Sun, S.X., Liu, G.G., 2003. Estimates and patterns of direct health care expenditures among individuals with back pain in the United States. Spine 29: 79-86.

Manchikanti, L., Singh, V., Datta, S., Cohen, S.P., Hirsch, J.A., 2009. Comprehensive review of epidemiology, scope, and impact of spinal pain. Pain Physician 12: e35-370.

Manchikanti, L., Helm, S., Fellows, B., janata, J.W., Pampati, V., Grider, J.S., Boswell, M.V., 2012. Pain Physician 15: es9-es38.

Mannion, A.F., Dolan, P., Adams, M.A., 1996. Psychological questionnaires: do "abnormal" scores precede or follow first-time low back pain? Spine 21: 2603-2611.

Marras, W.S., Lavender, S.A., Leurgans, S.E., Rajulu, S.L., Allread, W.G., Fathallah, F.A., Ferguson, S.A., 1993. The role of dynamic three-dimensional trunk motion in occupationallyrelated low back pain. The effects of workplace factors, trunk position, and trunk motion characteristics on risk of injury. Spine 18: 617-628. 
Marras, W.S., Granata, K.P., 1996. Spine loading during trunk lateral bending motions. Journal of Biomechanics 30: 697-703.

Marras, W.S., Jorgensen, M.J., Granata, K.P., Wiand, B., 2001. Female and male trunk geometry: size and prediction of the spine loading trunk muscles derived from MRI. Clinical Biomechanics 16: $38-46$.

Martin, B.I., Deyo, R.A., Mirza, S.K., Turner, J.A., Comstock, B.A., Hollingworth, W., Sullivan, S.D., 2012. Expenditures and health status among adults with back and neck problems. JAMA 13: $656-664$.

Martin, B.I., Turner, J.A., Mirza, S.K., Lee, M.J., Comstock, B.A., Deyo, R.A., 2009. Trends in health care expenditures, utilization, and health status among US adults with spine problems, 1997-2006. Spine 34: 2077-2084.

Mathiassen, S.E., Winkel, J., Hagg, G.M., 1995. Normalization of surface EMG amplitude from the upper trapezius muscle in ergonomics studies - a review. Journal of Electromyography and Kinesiology 5: 197-226.

Mathieu, P.A., Fortin, M., 2000. EMG and kinematics of normal subjects performing trunk flexion/extensions freely in space. Journal of Electromyography and Kinesiology 10: 197-209.

McAtamney, L., Corlett, E.N., 1993. RULA: a survey method for the investigation of workrelated upper limb disorders. Applied Ergonomics 24: 91-99.

Mezzarane, R.A., Kohn, A.F., 2007. Control of upright stance over inclined surfaces. Experimental brain research 180: 377-388.

McCully, K.K., Faulkner, J.A., 1985. Injury to skeletal muscle fibers of mice following lengthening contractions. Journal of Applied Physiology 59: 119-126. 
McGill, S.M., Kippers, V., 1994. Transfer of loads between lumbar tissues during the flexionrelaxation phenomenon. Spine 19: 2190-2196.

Mezzarane, R.A., Kohn, A.F., 2007. Control of upright stance over inclined surfaces. Experimental Brain Research 180: 377-388.

Mirka, G.A., Ning, X., Jin, S., Haddad, O., Kucera, K.L., 2011. Ergonomic interventions for commercial crab fishermen. International Journal of Industrial Ergonomics 41: 481-487.

Montgomery, D.C., 2012. Design and Analysis of Experiments. $7^{\text {th }}$ ed. New York: John Wiley and Sons.

Murray, R., Bohannon, R.W., Tiberio, D., Dewberry, M.J., Zannotti, C., 2002. Pelvifemoral rhythm during unilateral hip flexion in standing. Clinical Biomechanics 17: 147-151.

Muslim, K., Bazrgari, B., Hendershot, B., Toosizadeh, N., Nussbaum, M. A., Madigan, M. L. 2013. Disturbance and recovery of trunk mechanical and neuromuscular behaviors following repeated static trunk flexion: influences of duration and duty cycle on creep-induced effects. Applied Ergonomics 44: 643-651.

Nachemson, A.L., 1992. Newest knowledge of low back pain. A critical look. Cllinical Orthopaedics and Related Research 279: 8-20.

Patrick, N., Emanski, E., Knaub, M.A., 2016. Acute and Chronic Low Back Pain. Medical Clinics of North America 100: 169-181.

Neumann, W.P., Wells, R.P., Norman, R.W., Andrews, D.M., Frank, J., Shannon, H.S., Kerr, M.S., 1999. Comparison of four peak spinal loading exposure measurement methods and their association with low back pain. Scandinavian Journal of Work, Environment \& Health 25: 404409. 
Ning, X., Jin, S., Haddad, O., Mirka, G.A., 2011. Influence of asymmetry on the flexion relaxation response of the low back musculature. Clinical Biomechanics 26: 35-39.

Ning, X., Mirka, G.A., 2010. The Effect of Sinusoidal Rolling Ground Motion on Lifting Biomechanics. Applied Ergonomics 42:131-137.

NIOSH 1981. Work practices guide for manual lifting. NIOSH technical report No. 81-122, US Department of Health and Human Services, National Institute for Occupational Safety and Health, Cincinnati, OH.

Norman, R., Wells, R., Neumann, P., 1998. A comparison of peak vs cumulative physical work exposure risk factors for the reporting of low back pain in the automotive industry. Clinical Biomechanics 13: 561-573.

Oie, K.S., Kiemel, T., Jeka, J.J., 2002. Multisensory fusion: simultaneous re-weighting of vision and touch for the control of human posture. Cognitive Brain Research 14: 164-176.

Olson, M., Li, L., Solomonow, M., 2004. Flexion relaxation response to cyclic lumbar flexion. Clinical Biomechanics 19: 769-776.

Olson, M., Solomonow, M., Li, L., 2006. Flexion relaxation responses to gravity. Journal of Biomechanics 39: 2545-2554.

Panjabi, M.M., 1992a. The stabilizing system of the spine. Part I. Function, dysfunction, adaptation, and enhancement. Journal of Spinal Disorders 5: 383-389.

Panjabi, M.M., 1992b. The stabilizing system of the spine. Part ii. Neutral zone and instability hypothesis. Journal of Spinal Disorders 5: 390-396.

Patrick, N., Emanski, E., Knaub, M.A., 2016. Acute and chronic low back pain. Medical Clinics of North America 100: 169-181. 
Proske, U., Morgan, D.L., 2001. Muscle damage from eccentric exercise: mechanism, mechanical signs, adaptation and clinical application. Journal of Physiology 537: 333-345.

Punnett, L., Pruss-Ustun, A., Nelson, D.I., Fingerhut, M.A., Leigh, J., Tak, S., Phillips, S., 2005. Estimating the global burden of low back pain attributable to combined occupational exposures.

Reeves, N.P., Narendra, K.S., Cholewicki, J., 2007. Spine stability: the six blind men and the elephant. Clinical Biomechanics 22: 266-274.

Ricci, J.A., Stewart, W.F., Chee, E., Leotta, C., Foley, K., Hochberg, M.C., 2006. Spine 31: 3052-3060.

Ritzwoller, D.P., Crounse, L., Shetterly, S., Rublee, D., 2006. The association of comorbidities, utilization and costs for patients identified with low back pain. BMC Musculoskeletal Disorders 18: 7:72.

Sasagawa, S., Ushiyama, J., 2009. Balance control under different passive contributions of the ankle extensors: quiet standing on inclined surfaces. Experimental Brain Research 196: 537-544.

Schultz, A.B., Haderspeck-Grib, K., Sinkora, G., Warwick, D.N., 1985. Quantitative studies of the flexion-relaxation phenomenon in the back muscles. Journal of Orthopaedic Research 3: 189197.

Shin, G., Mirka, G., 2004. The effects of a sloped ground surface on trunk kinematics and L5/S1 moment during lifting. Ergonomics 15: 646-659.

Shmagel, A., Foley, R., Ibrahim, H., 2016. Epidemiology of chronic low back pain in US adults: National Health and Nutrition Examination Survey 2009-2010. Arthritis Care \& Research. DOI 10.1002/acr.22890

Sibley, K.M., Carpenter, M.G., Perry, J.C., Frank, J.S., 2007. Effects of postural anxiety on the soleus H-reflex. Human Movement Science 26: 103-112. 
Simeonov, P., Hsiao, H., Hendricks, S., 2009. Effectiveness of vertical visual reference for reducing postural instability on inclined and compliant surfaces at elevation. Applied Ergonomics 40: 353-361.

Simeonov, P.I., Hsiao, H., Dotson, B.W., Ammons, D.E., 2003. Control and perception of balance at elevated and sloped surfaces. Human Factors 45: 136-147.

Sitthipornvorakul, E., Janwantanakul, P., Purepong, N., Pensri, P., van der Beek, A.J., 2011. The association between physical activity and neck and low back pain: a systematic review. European Spine Journal 20: 677-689.

Social Security Administration, 2011. Annual Statistical Report on the Social Security Disability Insurance Program, 2011. Baltimore, MD: Office of Research Evaluation and Statistics. http://www.ssa.gov/policy/docs/statcomps/di asr/2011/di-asr11.pdf.

Sokal, R.R., Rohlf, F.J., 1982. Biometry: the principles and practice of statistics in biological research, $2^{\text {nd }}$ ed. Now York: Freeman.

Solomonow, M., Baratta, R.V., Banks, A., Freudenberger, C., Zhou, B.H., 2003. Flexion relaxation response to static lumbar flexion in males and females. Clinical Biomechanics 18 : 273-279.

Stanton, T.R., Henschke, N., Maher, C.G., Refshauge, K.M., Latimer, J., McAuley, J.H., 2008. After an episode of acute low back pain, recurrence is unpredictable and not as common as previously thought. Spine 33: 2923-2928.

Standring, S., 2015. Gray’s Anatomy (41th ed.). New York: Churchill Livingstone.

Taimela, S., Kankaanpaa, M., Luoto, S., 1999. The effect of lumbar fatigue on the ability to sense a change in lumbar position. A controlled study. Spine 24: 1322-1327. 
Tesch, P.A., Dudley, G.A., Duvoisin, M.R., hather, B.M., Harris, R.T., 1990. Force and EMG signal patterns during repeated bouts of concentric or eccentric muscle actions. Acta Physiologica Scandinavica Journal 138: 263-271.

Toussaint, H.M., de Winter, A.F., de Haas, Y., de Looze, M.P., Van Dieen, J.H., Kingma, I., 1995. Flexion relaxation during lifting: implications for torque production by muscle activity and tissue strain at the lumbo-sacral joint. Journal of Biomechanics 28: 199-210.

Urquhart, D.M., Barker, P.J., Hodges, P.W., Story, I.H., Briggs, C.A., 2005. Regional morphology of the transversus abdominis and obliquus internus and externus abdominis muscles. Clinical Biomechanics 20: 233-241.

Vleeming, A., Pool-Goudzwaard, A.I., Stoeckart, R., Wingerden, J.P., Snijders, C.J., 1995. The posterior layer of the thoracolumbar fascia: its function in load transfer from spine to legs. Spine 20: 753-758.

Vleeming, A., Pool-Goudzwaard, A.L., Hammudoghlu, D., Stoeckart, R., Snijders, C.J., Mens, J.M.A., 1996. The function of the long dorsal sacroiliac ligament. Its implication for understanding low back pain. Spine 21: 556-562.

Walker, B.F., Muller, R., Grant, W.D., 2003. Low back pain in Australian adults: the economic burden. Asia-Pacific Journal of Public Health 15: 79-87.

Wang, D., Dai, F., Ning, X., 2015. Risk assessment of work-related musculoskeletal disorders in construction: a state of the art review. Journal of Construction Engineering and Management. 10.1061/(ASCE)CO.1943-7862.0000979.

Ward, S.R., Kim, C.W., Eng, C.M., Gottschalk, L.J., Tomiya, A., Garfin, S.R., Lieber, R.L., 2009. Architectural analysis and intraoperative measurements demonstrate the unique design of the multifidus muscle for lumbar spine stability. The Journal of Bone and Joint Surgery 91: 176185. 
Waters, T.R., Putz-Anderson, V., Grag, A., Fine, L.J., 1993. Revised NIOSH equation for the design and evaluation of manual lifting tasks. Ergonomics 36: 749-776.

Wickel, E.E., Reiser, R.F., 2008. The effect of floor slope on sub-maximal lifting capacity and technique. Applied Ergonomics 39: 296-304.

Wingerden, J.P., Vleeming, A., Snijderes, C.J., Stoeckart, R., 1993. A functional anatomical approach to the spine-pelvis mechanism: interaction between the biceps femoris muscle and the sacrotuberous ligament. European Spine Journal 2: 140-144.

Leboeuf-Yde, C., Kyvik, K.O., Bruun, N.H., 1998. Low back pain and lifestyle part I: smoking, information from a population based sample of 29424 twins. Spine 23: 2207-2214.

Zhao, Y., Upadhyaya, S.K., Kaminaka, M.S., 1987. Foot-ground forces on sloping ground when lifting. Ergonomics 30: 1671-1678. 


\section{APPENDIX A. COMPLETE ANOVA TABLES}

\section{General Linear Model: MDIST C7, MDIST AP C7, ... versus Subject, GROUND, ...}

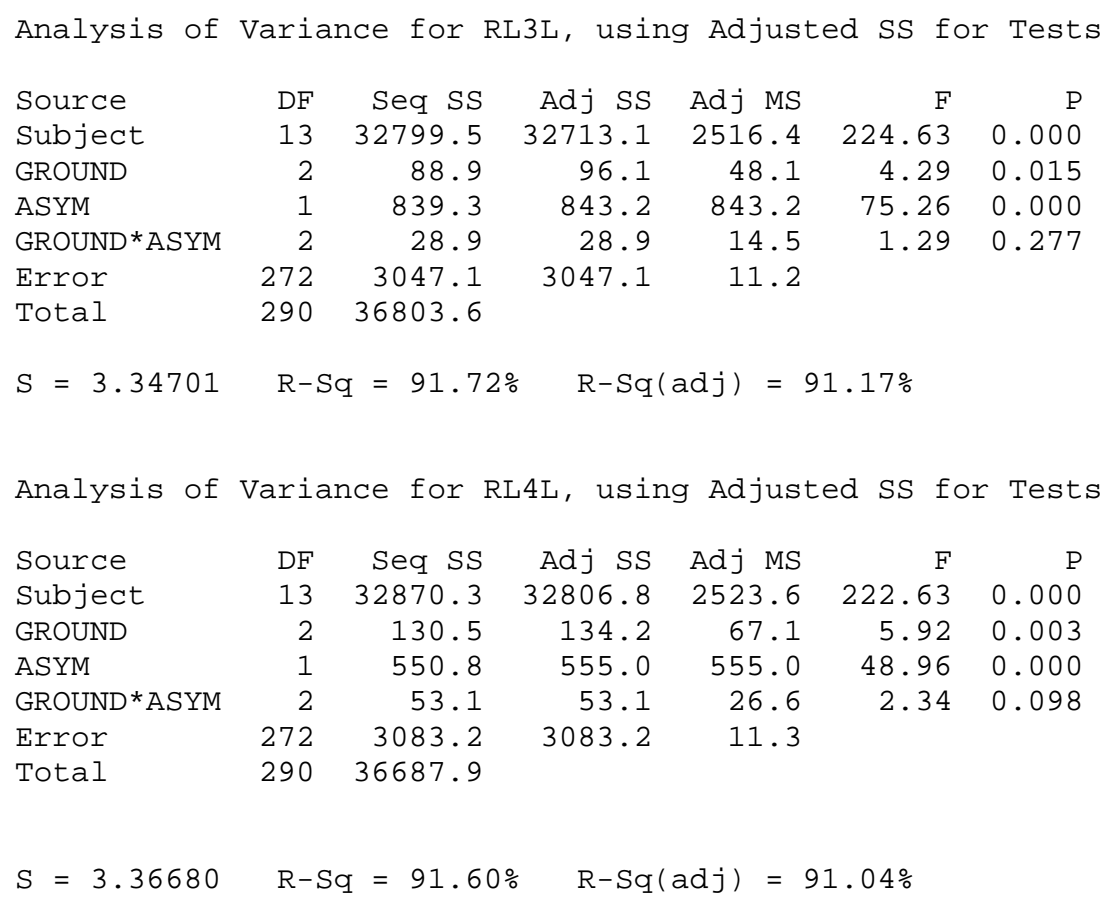




$\begin{array}{lrrrrrr}\text { Source } & \text { DF } & \text { Seq SS } & \text { Adj SS } & \text { Adj MS } & \text { F } & \text { P } \\ \text { Subject } & 13 & 30184.2 & 30109.3 & 2316.1 & 1209.64 & 0.000 \\ \text { GROUND } & 2 & 14.1 & 13.7 & 6.9 & 3.59 & 0.029 \\ \text { ASYM } & 1 & 35.0 & 34.9 & 34.9 & 18.21 & 0.000 \\ \text { GROUND*ASYM } & 2 & 8.5 & 8.5 & 4.2 & 2.22 & 0.111 \\ \text { Error } & 272 & 520.8 & 520.8 & 1.9 & & \\ \text { Total } & 290 & 30762.6 & & & & \\ \text { S }=1.38373 & \text { R-Sq }=98.31 \% & \text { R-Sq (adj) }=98.20 \% & \end{array}$

Analysis of Variance for MTI, using Adjusted SS for Tests

$\begin{array}{lrrrrrr}\text { Source } & \text { DF } & \text { Seq SS } & \text { Adj SS } & \text { Adj MS } & \text { F } & \text { P } \\ \text { Subject } & 13 & 15763.50 & 15246.93 & 1172.84 & 116.42 & 0.000 \\ \text { GROUND } & 2 & 14.96 & 22.54 & 11.27 & 1.12 & 0.328 \\ \text { ASYM } & 1 & 1255.47 & 1238.95 & 1238.95 & 122.99 & 0.000 \\ \text { GROUND*ASYM } & 2 & 61.47 & 61.47 & 30.74 & 3.05 & 0.049 \\ \text { Error } & 272 & 2740.09 & 2740.09 & 10.07 & & \\ \text { Total } & 290 & 19835.48 & & & & \\ \text { S } 3.17393 & \text { R-Sq }=86.19 \% & \text { R-Sq(adj) }=85.27 \% & & \end{array}$

$\begin{array}{lrrrrrr}\text { Analysis of Variance for MDIST C7, using Adjusted SS for } & \text { Tests } \\ \text { Source } & \text { DF } & \text { Seq SS } & \text { Adj SS } & \text { Adj MS } & \text { F } & \text { P } \\ \text { Subject } & 13 & 0.0022720 & 0.0022720 & 0.0001748 & 15.16 & 0.000 \\ \text { GROUND } & 2 & 0.0000447 & 0.0000447 & 0.0000223 & 1.94 & 0.146 \\ \text { ASYM } & 1 & 0.0001511 & 0.0001511 & 0.0001511 & 13.11 & 0.000 \\ \text { GROUND*ASYM } & 2 & 0.0000156 & 0.0000156 & 0.0000078 & 0.68 & 0.509 \\ \text { Error } & 317 & 0.0036548 & 0.0036548 & 0.0000115 & & \\ \text { Total } & 335 & 0.0061382 & & & & \\ \text { S }=0.00339547 & \text { R-Sq }=40.46 \% & R-S q(\operatorname{adj})=37.08 \% & \end{array}$

\begin{tabular}{|c|c|c|c|c|c|c|}
\hline Source & $\mathrm{DF}$ & Seq SS & Adj $S S$ & Adj MS & $\mathrm{F}$ & P \\
\hline Subject & 13 & 0.0012075 & 0.0012075 & 0.0000929 & 10.81 & 0.000 \\
\hline GROUND & 2 & 0.0000249 & 0.0000249 & 0.0000124 & 1.45 & 0.237 \\
\hline ASYM & 1 & 0.0001570 & 0.0001570 & 0.0001570 & 18.27 & 0.000 \\
\hline GROUND *ASYM & 2 & 0.0000204 & 0.0000204 & 0.0000102 & 1.19 & 0.307 \\
\hline Error & 317 & 0.0027235 & 0.0027235 & 0.0000086 & & \\
\hline Total & 335 & 0.0041332 & & & & \\
\hline
\end{tabular}

Analysis of Variance for MDIST ML C7, using Adjusted SS for Tests

$\begin{array}{lrrrrrr}\text { Source } & \text { DF } & \text { Seq SS } & \text { Adj SS } & \text { Adj MS } & \text { F } & \text { P } \\ \text { Subject } & 13 & 0.0008149 & 0.0008149 & 0.0000627 & 9.29 & 0.000 \\ \text { GROUND } & 2 & 0.0000286 & 0.0000286 & 0.0000143 & 2.12 & 0.122 \\ \text { ASYM } & 1 & 0.0000134 & 0.0000134 & 0.0000134 & 1.99 & 0.160 \\ \text { GROUND*ASYM } & 2 & 0.0000074 & 0.0000074 & 0.0000037 & 0.55 & 0.578 \\ \text { Error } & 317 & 0.0021384 & 0.0021384 & 0.0000067 & & \\ \text { Total } & 335 & 0.0030027 & & & & \end{array}$

$\mathrm{S}=0.00259727 \quad \mathrm{R}-\mathrm{Sq}=28.78 \% \quad \mathrm{R}-\mathrm{Sq}(\mathrm{adj})=24.74 \%$ 
Analysis of Variance for RDIST C7, using Adjusted SS for Tests

$\begin{array}{lrrrrrr}\text { Source } & \text { DF } & \text { Seq SS } & \text { Adj SS } & \text { Adj MS } & \text { F } & P \\ \text { Subject } & 13 & 1.201071 & 1.201071 & 0.092390 & 14.92 & 0.000 \\ \text { GROUND } & 2 & 0.023528 & 0.023528 & 0.011764 & 1.90 & 0.151 \\ \text { ASYM } & 1 & 0.085670 & 0.085670 & 0.085670 & 13.83 & 0.000 \\ \text { GROUND ASYM } & 2 & 0.005598 & 0.005598 & 0.002799 & 0.45 & 0.637 \\ \begin{array}{l}\text { Error } \\ \text { Total }\end{array} & 317 & 1.963202 & 1.963202 & 0.006193 & & \\ \text { S }=0.0786960 & \text { R-Sq }=40.13 \% & 3.279069 & & & & \\ \end{array}$

Analysis of Variance for RDIST AP C7, using Adjusted SS for Tests

$\begin{array}{lrrrrrr}\text { Source } & \text { DF } & \text { Seq SS } & \text { Adj SS } & \text { Adj MS } & \text { F } & \text { P } \\ \text { Subject } & 13 & 0.688807 & 0.688807 & 0.052985 & 11.43 & 0.000 \\ \text { GROUND } & 2 & 0.011881 & 0.011881 & 0.005941 & 1.28 & 0.279 \\ \text { ASYM } & 1 & 0.097672 & 0.097672 & 0.097672 & 21.07 & 0.000 \\ \text { GROUND*ASYM } & 2 & 0.011291 & 0.011291 & 0.005645 & 1.22 & 0.297 \\ \text { Error } & 317 & 1.469375 & 1.469375 & 0.004635 & & \\ \text { Total } & 335 & 2.279026 & & & & \\ \text { S }=0.0680827 & \text { R-Sq }=35.53 \% & R-S q(\text { adj) }=31.87 \% & \end{array}$

Analysis of Variance for RDIST ML C7, using Adjusted SS for Tests

$\begin{array}{lrrrrrr}\text { Source } & \text { DF } & \text { Seq SS } & \text { Adj SS } & \text { Adj MS } & F & P \\ \text { Subject } & 13 & 0.485060 & 0.485060 & 0.037312 & 9.17 & 0.000 \\ \text { GROUND } & 2 & 0.018452 & 0.018452 & 0.009226 & 2.27 & 0.105 \\ \text { ASYM } & 1 & 0.008435 & 0.008435 & 0.008435 & 2.07 & 0.151 \\ \text { GROUND*ASYM } & 2 & 0.002639 & 0.002639 & 0.001320 & 0.32 & 0.723 \\ \text { Error } & 317 & 1.289307 & 1.289307 & 0.004067 & & \\ \text { Total } & 335 & 1.803894 & & & & \\ \text { S }=0.0637747 & \mathrm{R}-\mathrm{Sq}=28.53 \% & \mathrm{R}-\mathrm{Sq}(\text { adj) }=24.47 \% & \end{array}$

$\begin{array}{lrrrrrrr}\text { Analysis of } & \text { Variance for TOTEX C7, using Adjusted SS for Tests } \\ \text { Source } & \text { DF } & \text { Seq SS } & \text { Adj SS } & \text { Adj MS } & \text { F } & \text { P } \\ \text { Subject } & 13 & 0.0729850 & 0.0729850 & 0.0056142 & 27.41 & 0.000 \\ \text { GROUND } & 2 & 0.0049861 & 0.0049861 & 0.0024930 & 12.17 & 0.000 \\ \text { ASYM } & 1 & 0.0000014 & 0.0000014 & 0.0000014 & 0.01 & 0.933 \\ \text { GROUND*ASYM } & 2 & 0.0005632 & 0.0005632 & 0.0002816 & 1.37 & 0.254 \\ \text { Error } & 317 & 0.0649371 & 0.0649371 & 0.0002048 & & \\ \text { Total } & 335 & 0.1434728 & & & & \end{array}$

$$
S=0.0143125 \quad R-S q=54.74 \% \quad R-S q(\operatorname{adj})=52.17 \%
$$

Analysis of Variance for TOTEX AP C7, using Adjusted SS for Tests

$\begin{array}{lrrrrrr}\text { Source } & \text { DF } & \text { Seq SS } & \text { Adj SS } & \text { Adj MS } & \text { F } & \text { P } \\ \text { Subject } & 13 & 0.0338738 & 0.0338738 & 0.0026057 & 21.25 & 0.000 \\ \text { GROUND } & 2 & 0.0013526 & 0.0013526 & 0.0006763 & 5.51 & 0.004 \\ \text { ASYM } & 1 & 0.0005981 & 0.0005981 & 0.0005981 & 4.88 & 0.028 \\ \text { GROUND*ASYM } & 2 & 0.0003985 & 0.0003985 & 0.0001992 & 1.62 & 0.199 \\ \text { Error } & 317 & 0.0388731 & 0.0388731 & 0.0001226 & & \\ \text { Total } & 335 & 0.0750961 & & & & \end{array}$


$S=0.0110738 \quad R-S q=48.24 \% \quad R-S q(\operatorname{adj})=45.30 \%$

Analysis of Variance for TOTEX ML C7, using Adjusted SS for Tests

\begin{tabular}{|c|c|c|c|c|c|c|}
\hline Source & $\mathrm{DF}$ & Seq SS & Adj $S S$ & Adj MS & $\mathrm{F}$ & $\mathrm{P}$ \\
\hline Subject & 13 & 0.0296961 & 0.0296961 & 0.0022843 & 17.79 & 0.000 \\
\hline GROUND & 2 & 0.0030884 & 0.0030884 & 0.0015442 & 12.02 & 0.000 \\
\hline ASYM & 1 & 0.0006531 & 0.0006531 & 0.0006531 & 5.09 & 0.025 \\
\hline GROUND *ASYM & 2 & 0.0002343 & 0.0002343 & 0.0001172 & 0.91 & 0.403 \\
\hline Error & 317 & 0.0407111 & 0.0407111 & 0.0001284 & & \\
\hline Total & 335 & 0.0743830 & & & & \\
\hline
\end{tabular}

Analysis of Variance for MVELO C7, using Adjusted SS for Tests

$\begin{array}{lrrrrrr}\text { Source } & \text { DF } & \text { Seq SS } & \text { Adj SS } & \text { Adj MS } & \text { F } & P \\ \text { Subject } & 13 & 0.0038117 & 0.0038117 & 0.0002932 & 29.04 & 0.000 \\ \text { GROUND } & 2 & 0.0002408 & 0.0002408 & 0.0001204 & 11.93 & 0.000 \\ \text { ASYM } & 1 & 0.0000001 & 0.0000001 & 0.0000001 & 0.01 & 0.937 \\ \text { GROUND*ASYM } & 2 & 0.0000284 & 0.0000284 & 0.0000142 & 1.40 & 0.247 \\ \text { Error } & 317 & 0.0032002 & 0.0032002 & 0.0000101 & & \\ \text { Total } & 335 & 0.0072811 & & & & \\ \text { S }=0.00317732 & \text { R-Sq }=56.05 \% & R-S q(\operatorname{adj})=53.55 \% & \end{array}$

Analysis of Variance for MVELO AP C7, using Adjusted SS for Tests

$\begin{array}{lrrrrrr}\text { Source } & \text { DF } & \text { Seq SS } & \text { Adj SS } & \text { Adj MS } & \text { F } & \text { P } \\ \text { Subject } & 13 & 0.0017431 & 0.0017431 & 0.0001341 & 22.18 & 0.000 \\ \text { GROUND } & 2 & 0.0000656 & 0.0000656 & 0.0000328 & 5.43 & 0.005 \\ \text { ASYM } & 1 & 0.0000292 & 0.0000292 & 0.0000292 & 4.83 & 0.029 \\ \text { GROUND*ASYM } & 2 & 0.0000202 & 0.0000202 & 0.0000101 & 1.67 & 0.190 \\ \text { Error } & 317 & 0.0019162 & 0.0019162 & 0.0000060 & & \\ \text { Total } & 335 & 0.0037742 & & & & \end{array}$

$$
\mathrm{S}=0.00245861 \quad \mathrm{R}-\mathrm{Sq}=49.23 \% \mathrm{R}-\mathrm{Sq}(\operatorname{adj})=46.35 \%
$$

Analysis of Variance for MVELO ML C7, using Adjusted SS for Tests

$\begin{array}{lrrrrrr}\text { Source } & \text { DF } & \text { Seq SS } & \text { Adj SS } & \text { Adj MS } & \text { F } & P \\ \text { Subject } & 13 & 0.0015645 & 0.0015645 & 0.0001203 & 19.02 & 0.000 \\ \text { GROUND } & 2 & 0.0001490 & 0.0001490 & 0.0000745 & 11.77 & 0.000 \\ \text { ASYM } & 1 & 0.0000320 & 0.0000320 & 0.0000320 & 5.06 & 0.025 \\ \text { GROUND*ASYM } & 2 & 0.0000117 & 0.0000117 & 0.0000059 & 0.92 & 0.398 \\ \text { Error } & 317 & 0.0020061 & 0.0020061 & 0.0000063 & & \\ \text { Total } & 335 & 0.0037633 & & & & \\ \text { S }=0.00251564 & \text { R-Sq }=46.69 \% & R-S q(\text { adj })=43.67 \% & & \end{array}$

Analysis of Variance for AREA CC C7, using Adjusted SS for Tests

$\begin{array}{lrrrrrr}\text { Source } & \text { DF } & \text { Seq SS } & \text { Adj SS } & \text { Adj MS } & F & P \\ \text { Subject } & 13 & 21.8469 & 21.8469 & 1.6805 & 9.43 & 0.000 \\ \text { GROUND } & 2 & 0.5302 & 0.5302 & 0.2651 & 1.49 & 0.228 \\ \text { ASYM } & 1 & 1.8568 & 1.8568 & 1.8568 & 10.42 & 0.001 \\ \text { GROUND*ASYM } & 2 & 0.6517 & 0.6517 & 0.3258 & 1.83 & 0.162 \\ \text { Error } & 317 & 56.4984 & 56.4984 & 0.1782 & & \end{array}$




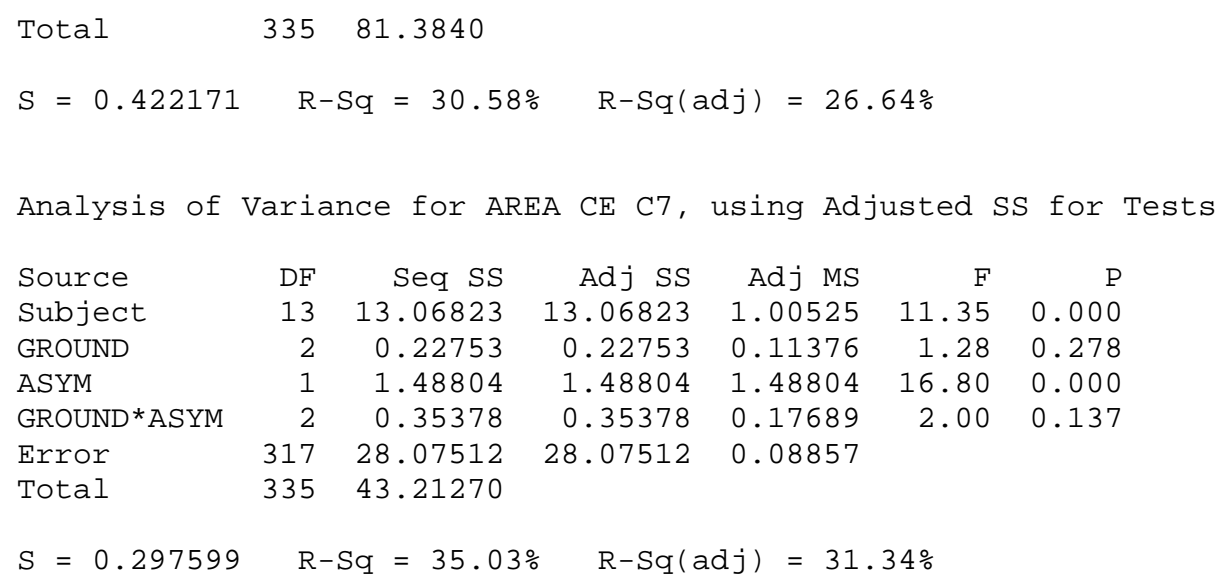

Analysis of Variance for MDIST ML T12, using Adjusted SS for Tests

$\begin{array}{lrrrrrr}\text { Source } & \text { DF } & \text { Seq SS } & \text { Adj SS } & \text { Adj MS } & \text { F } & \text { P } \\ \text { Subject } & 13 & 0.0012029 & 0.0012029 & 0.0000925 & 12.69 & 0.000 \\ \text { GROUND } & 2 & 0.0000298 & 0.0000298 & 0.0000149 & 2.04 & 0.131 \\ \text { ASYM } & 1 & 0.0000052 & 0.0000052 & 0.0000052 & 0.72 & 0.397\end{array}$




$\begin{array}{lrrrrrr}\text { GROUND*ASYM } & 2 & 0.0000067 & 0.0000067 & 0.0000033 & 0.46 & 0.633 \\ \begin{array}{l}\text { Error } \\ \text { Total }\end{array} & 317 & 0.0023120 & 0.0023120 & 0.0000073 & & \\ \text { S }=0.00270062 & \text { R-Sq }=34.99 \% & 0.0035566 & \text { R-Sq (adj) }=31.30 \%\end{array}$

Analysis of Variance for RDIST T12, using Adjusted SS for Tests

$\begin{array}{lrrrrrr}\text { Source } & \text { DF } & \text { Seq SS } & \text { Adj SS } & \text { Adj MS } & F & P \\ \text { Subject } & 13 & 2.28601 & 2.28601 & 0.17585 & 17.34 & 0.000 \\ \text { GROUND } & 2 & 0.03674 & 0.03674 & 0.01837 & 1.81 & 0.165 \\ \text { ASYM } & 1 & 0.06937 & 0.06937 & 0.06937 & 6.84 & 0.009 \\ \text { GROUND*ASYM } & 2 & 0.00101 & 0.00101 & 0.00051 & 0.05 & 0.951 \\ \text { Error } & 317 & 3.21505 & 3.21505 & 0.01014 & & \\ \text { Total } & 335 & 5.60819 & & & & \end{array}$

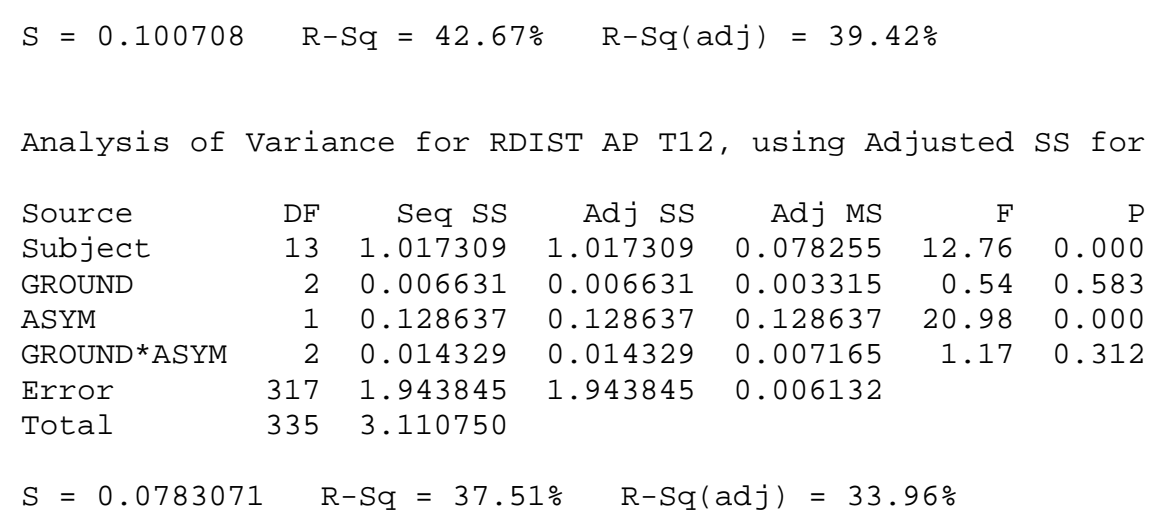

Analysis of Variance for RDIST ML T12, using Adjusted SS for Tests

$\begin{array}{lrrrrrr}\text { Source } & \text { DF } & \text { Seq SS } & \text { Adj SS } & \text { Adj MS } & F & P \\ \text { Subject } & 13 & 1.244135 & 1.244135 & 0.095703 & 11.82 & 0.000 \\ \text { GROUND } & 2 & 0.037955 & 0.037955 & 0.018978 & 2.34 & 0.098 \\ \text { ASYM } & 1 & 0.001672 & 0.001672 & 0.001672 & 0.21 & 0.650 \\ \text { GROUND*ASYM } & 2 & 0.004717 & 0.004717 & 0.002359 & 0.29 & 0.747 \\ \text { Error } & 317 & 2.566369 & 2.566369 & 0.008096 & & \\ \text { Total } & 335 & 3.854849 & & & & \\ \text { S }=0.0899767 & \text { R-Sq }=33.42 \% & R-S q(\text { adj) }=29.64 \% & \end{array}$

Analysis of Variance for TOTEX T12, using Adjusted SS for Tests

$\begin{array}{lrrrrrr}\text { Source } & \text { DF } & \text { Seq SS } & \text { Adj SS } & \text { Adj MS } & \text { F } & P \\ \text { Subject } & 13 & 0.0876592 & 0.0876592 & 0.0067430 & 31.88 & 0.000 \\ \text { GROUND } & 2 & 0.0040704 & 0.0040704 & 0.0020352 & 9.62 & 0.000 \\ \text { ASYM } & 1 & 0.0000050 & 0.0000050 & 0.0000050 & 0.02 & 0.878 \\ \text { GROUND*ASYM } & 2 & 0.0009637 & 0.0009637 & 0.0004819 & 2.28 & 0.104 \\ \begin{array}{l}\text { Error } \\ \text { Total }\end{array} & 317 & 0.0670545 & 0.0670545 & 0.0002115 & & \\ \text { S }=0.0145440 & \text { R-Sq }=58.03 \% & 0.1597528 & & & & \\ & & & & & & \end{array}$

Analysis of Variance for TOTEX AP T12, using Adjusted SS for Tests

$\begin{array}{lllllll}\text { Source } & \text { DF } & \text { Seq SS } & \text { Adj SS } & \text { Adj MS } & \text { F }\end{array}$

$\begin{array}{lllllll}\text { Subject } & 13 & 0.0246112 & 0.0246112 & 0.0018932 & 25.62 & 0.000\end{array}$ 


$\begin{array}{lrrrrrr}\text { GROUND } & 2 & 0.0009425 & 0.0009425 & 0.0004712 & 6.38 & 0.002 \\ \text { ASYM } & 1 & 0.0012351 & 0.0012351 & 0.0012351 & 16.71 & 0.000 \\ \text { GROUND*ASYM } & 2 & 0.0002247 & 0.0002247 & 0.0001123 & 1.52 & 0.220 \\ \text { Error } & 317 & 0.0234253 & 0.0234253 & 0.0000739 & & \\ \text { Total } & 335 & 0.0504388 & & & & \end{array}$

$$
S=0.00859633 \quad R-S q=53.56 \% \quad R-S q(\operatorname{adj})=50.92 \%
$$

Analysis of Variance for TOTEX ML T12, using Adjusted SS for Tests

$\begin{array}{lrrrrrr}\text { Source } & \text { DF } & \text { Seq SS } & \text { Adj SS } & \text { Adj MS } & F & P \\ \text { Subject } & 13 & 0.0565423 & 0.0565423 & 0.0043494 & 25.56 & 0.000 \\ \text { GROUND } & 2 & 0.0027223 & 0.0027223 & 0.0013612 & 8.00 & 0.000 \\ \text { ASYM } & 1 & 0.0006088 & 0.0006088 & 0.0006088 & 3.58 & 0.059 \\ \text { GROUND ASYM } & 2 & 0.0006349 & 0.0006349 & 0.0003175 & 1.87 & 0.156 \\ \text { Error } & 317 & 0.0539318 & 0.0539318 & 0.0001701 & & \\ \text { Total } & 335 & 0.1144403 & & & & \\ \text { S }=0.0130435 & \text { R-Sq }=52.87 \% & R-S q(\text { adj })=50.20 \% & & \end{array}$

$\begin{array}{lrrrrrr}\text { Analysis of Variance for MVELO T12, using Adjusted SS for } & \text { Tests } \\ \text { Source } & \text { DF } & \text { Seq SS } & \text { Adj SS } & \text { Adj MS } & \text { F } & \text { P } \\ \text { Subject } & 13 & 0.0044913 & 0.0044913 & 0.0003455 & 33.19 & 0.000 \\ \text { GROUND } & 2 & 0.0001961 & 0.0001961 & 0.0000980 & 9.42 & 0.000 \\ \text { ASYM } & 1 & 0.0000002 & 0.0000002 & 0.0000002 & 0.02 & 0.886 \\ \text { GROUND*ASYM } & 2 & 0.0000476 & 0.0000476 & 0.0000238 & 2.28 & 0.104 \\ \text { Error } & 317 & 0.0032998 & 0.0032998 & 0.0000104 & & \\ \text { Total } & 335 & 0.0080350 & & & & \\ \text { S }=0.00322637 & \text { R-Sq }=58.93 \% & R-S q(\text { adj })=56.60 \% & \end{array}$

Analysis of Variance for MVELO AP T12, using Adjusted SS for Tests

$\begin{array}{lrrrrrr}\text { Source } & \text { DF } & \text { Seq SS } & \text { Adj SS } & \text { Adj MS } & \text { F } & P \\ \text { Subject } & 13 & 0.0012569 & 0.0012569 & 0.0000967 & 26.59 & 0.000 \\ \text { GROUND } & 2 & 0.0000457 & 0.0000457 & 0.0000228 & 6.28 & 0.002 \\ \text { ASYM } & 1 & 0.0000600 & 0.0000600 & 0.0000600 & 16.51 & 0.000 \\ \text { GROUND*ASYM } & 2 & 0.0000111 & 0.0000111 & 0.0000056 & 1.53 & 0.218 \\ \text { Error } & 317 & 0.0011526 & 0.0011526 & 0.0000036 & & \\ \text { Total } & 335 & 0.0025263 & & & & \\ \text { S }=0.00190685 & \text { R-Sq }=54.37 \% & R-S q(\text { adj })=51.78 \% & \end{array}$

Analysis of Variance for MVELO ML T12, using Adjusted SS for Tests

$\begin{array}{lrrrrrr}\text { Source } & \text { DF } & \text { Seq SS } & \text { Adj SS } & \text { Adj MS } & \text { F } & \text { P } \\ \text { Subject } & 13 & 0.0028830 & 0.0028830 & 0.0002218 & 26.49 & 0.000 \\ \text { GROUND } & 2 & 0.0001310 & 0.0001310 & 0.0000655 & 7.82 & 0.000 \\ \text { ASYM } & 1 & 0.0000299 & 0.0000299 & 0.0000299 & 3.58 & 0.059 \\ \text { GROUND*ASYM } & 2 & 0.0000313 & 0.0000313 & 0.0000157 & 1.87 & 0.156 \\ \text { Error } & 317 & 0.0026536 & 0.0026536 & 0.0000084 & & \\ \text { Total } & 335 & 0.0057289 & & & & \end{array}$

$$
S=0.00289328 \quad R-S q=53.68 \% \quad R-S q(\operatorname{adj})=51.05 \%
$$

Analysis of Variance for AREA CC T12, using Adjusted SS for Tests 


$\begin{array}{lrrrrrr}\text { Source } & \text { DF } & \text { Seq SS } & \text { Adj SS } & \text { Adj MS } & F & P \\ \text { Subject } & 13 & 67.4314 & 67.4314 & 5.1870 & 11.59 & 0.000 \\ \text { GROUND } & 2 & 0.4967 & 0.4967 & 0.2483 & 0.55 & 0.575 \\ \text { ASYM } & 1 & 2.2850 & 2.2850 & 2.2850 & 5.10 & 0.025 \\ \text { GROUND*ASYM } & 2 & 0.4849 & 0.4849 & 0.2424 & 0.54 & 0.582 \\ \text { Error } & 317 & 141.8882 & 141.8882 & 0.4476 & & \\ \text { Total } & 335 & 212.5861 & & & & \\ \text { S }=0.669027 & \text { R-Sq }=33.26 \% & \text { R-Sq(adj) }=29.47 \% & \end{array}$

\begin{tabular}{|c|c|c|c|c|c|c|c|}
\hline Source & $\mathrm{DF}$ & Seq SS & Adj $S S$ & dj $\mathrm{MS}$ & $\mathrm{F}$ & & P \\
\hline Subject & 13 & 41.9762 & 41.9762 & 3.2289 & 13.34 & 0.000 & \\
\hline GROUND & 2 & 0.0829 & 0.0829 & .0415 & 0.17 & 0.843 & \\
\hline ASYM & 1 & 2.7420 & 2.7420 & .7420 & 11.33 & 0.001 & \\
\hline GROUND *ASYM & 2 & 0.2996 & 0.2996 & .1498 & 0.62 & 0.539 & \\
\hline Error & 317 & 76.7205 & 76.7205 & .2420 & & & \\
\hline Total & 335 & 121.8212 & & & & & \\
\hline$S=0.491956$ & $\mathrm{R}-$ & $\mathrm{Sq}=37.02 \%$ & $\mathrm{R}-\mathrm{Sq}(\mathrm{ad})$ & $(j)=3$ & $3.45 \%$ & & \\
\hline Analysis of & Varia & nce for AREP & $\mathrm{A}$ SW T12, & using & Adjuste & ed SS $f$ & for $T \in$ \\
\hline Source & $\mathrm{DF}$ & Seq SS & $\operatorname{Adj} S S$ & & $d j \mathrm{MS}$ & $F$ & $\mathrm{~F}$ \\
\hline Subject & 13 & 0.0000443 & 0.0000443 & 0.00 & 00034 & 15.22 & 0.000 \\
\hline GROUND & 2 & 0.0000006 & 0.0000006 & 0.00 & 00003 & 1.29 & 0.27 \\
\hline ASYM & 1 & 0.0000008 & 0.0000008 & 0.00 & 00008 & 3.37 & 0.06 \\
\hline GROUND *ASYM & 2 & 0.0000002 & 0.0000002 & 0.00 & 00001 & 0.36 & 0.69 \\
\hline Error & 317 & 0.0000709 & 0.0000709 & 0.00 & 00002 & & \\
\hline Total & 335 & 0.0001167 & & & & & \\
\hline
\end{tabular}

$\begin{array}{lrrrrrr}\text { Analysis of Variance for MDIST } & \text { S1, using Adjusted SS for } & \text { Tests } \\ \text { Source } & \text { DF } & \text { Seq SS } & \text { Adj SS } & \text { Adj MS } & \text { F } & \text { P } \\ \text { Subject } & 13 & 0.0010909 & 0.0010909 & 0.0000839 & 15.98 & 0.000 \\ \text { GROUND } & 2 & 0.0000308 & 0.0000308 & 0.0000154 & 2.93 & 0.055 \\ \text { ASYM } & 1 & 0.0000001 & 0.0000001 & 0.0000001 & 0.01 & 0.904 \\ \text { GROUND*ASYM } & 2 & 0.0000027 & 0.0000027 & 0.0000014 & 0.26 & 0.773 \\ \text { Error } & 317 & 0.0016650 & 0.0016650 & 0.0000053 & & \\ \text { Total } & 335 & 0.0027895 & & & & \\ \text { S }=0.00229182 & \text { R-Sq }=40.31 \% & R-S q(\operatorname{adj})=36.92 \%\end{array}$

Analysis of Variance for MDIST AP S1, using Adjusted SS for Tests

$\begin{array}{lrrrrrr}\text { Source } & \text { DF } & \text { Seq SS } & \text { Adj SS } & \text { Adj MS } & \text { F } & P \\ \text { Subject } & 13 & 0.0003985 & 0.0003985 & 0.0000307 & 14.99 & 0.000 \\ \text { GROUND } & 2 & 0.0000143 & 0.0000143 & 0.0000072 & 3.50 & 0.031 \\ \text { ASYM } & 1 & 0.0000145 & 0.0000145 & 0.0000145 & 7.07 & 0.008 \\ \text { GROUND*ASYM } & 2 & 0.0000002 & 0.0000002 & 0.0000001 & 0.05 & 0.947 \\ \text { Error } & 317 & 0.0006481 & 0.0006481 & 0.0000020 & & \\ \text { Total } & 335 & 0.0010756 & & & & \\ \text { S }=0.00142981 & \text { R-Sq }=39.75 \% & R-S q(\text { adj })=36.33 \% & & \end{array}$


Analysis of Variance for MDIST ML S1, using Adjusted SS for Tests

$\begin{array}{lrrrrrr}\text { Source } & \text { DF } & \text { Seq SS } & \text { Adj SS } & \text { Adj MS } & \text { F } & \text { P } \\ \text { Subject } & 13 & 0.0006894 & 0.0006894 & 0.0000530 & 11.76 & 0.000 \\ \text { GROUND } & 2 & 0.0000094 & 0.0000094 & 0.0000047 & 1.04 & 0.353 \\ \text { ASYM } & 1 & 0.0000088 & 0.0000088 & 0.0000088 & 1.95 & 0.164 \\ \text { GROUND*ASYM } & 2 & 0.0000043 & 0.0000043 & 0.0000021 & 0.47 & 0.624 \\ \text { Error } & 317 & 0.0014291 & 0.0014291 & 0.0000045 & & \\ \text { Total } & 335 & 0.0021410 & & & & \\ \text { S }=0.00212322 & \text { R-Sq }=33.25 \% & \text { R-Sq(adj) }=29.46 \%\end{array}$

Analysis of Variance for RDIST S1, using Adjusted SS for Tests

$\begin{array}{lrrrrrr}\text { Source } & \text { DF } & \text { Seq SS } & \text { Adj SS } & \text { Adj MS } & \text { F } & P \\ \text { Subject } & 13 & 2.88919 & 2.88919 & 0.22225 & 17.79 & 0.000 \\ \text { GROUND } & 2 & 0.03989 & 0.03989 & 0.01994 & 1.60 & 0.204 \\ \text { ASYM } & 1 & 0.05806 & 0.05806 & 0.05806 & 4.65 & 0.032 \\ \text { GROUND*ASYM } & 2 & 0.00023 & 0.00023 & 0.00011 & 0.01 & 0.991 \\ \text { Error } & 317 & 3.96085 & 3.96085 & 0.01249 & & \\ \text { Total } & 335 & 6.94821 & & & & \\ \text { S }=0.111780 & \mathrm{R}-\mathrm{Sq}=42.99 \% & \mathrm{R}-\mathrm{Sq}(\operatorname{adj})=39.76 \% & \end{array}$

Analysis of Variance for RDIST AP S1, using Adjusted SS for Tests

$\begin{array}{lrrrrrr}\text { Source } & \text { DF } & \text { Seq SS } & \text { Adj SS } & \text { Adj MS } & \text { F } & \text { P } \\ \text { Subject } & 13 & 1.248979 & 1.248979 & 0.096075 & 14.04 & 0.000 \\ \text { GROUND } & 2 & 0.006275 & 0.006275 & 0.003137 & 0.46 & 0.633 \\ \text { ASYM } & 1 & 0.140888 & 0.140888 & 0.140888 & 20.58 & 0.000 \\ \text { GROUND*ASYM } & 2 & 0.013755 & 0.013755 & 0.006878 & 1.00 & 0.367 \\ \text { Error } & 317 & 2.169883 & 2.169883 & 0.006845 & & \\ \text { Total } & 335 & 3.579781 & & & & \end{array}$

$S=0.0827349 \quad R-S q=39.39 \% \quad R-S q(\operatorname{adj})=35.94 \%$

Analysis of Variance for RDIST ML S1, using Adjusted SS for Tests

$\begin{array}{lrrrrrr}\text { Source } & \text { DF } & \text { Seq SS } & \text { Adj SS } & \text { Adj MS } & F & P \\ \text { Subject } & 13 & 1.65170 & 1.65170 & 0.12705 & 12.23 & 0.000 \\ \text { GROUND } & 2 & 0.04092 & 0.04092 & 0.02046 & 1.97 & 0.141 \\ \text { ASYM } & 1 & 0.00013 & 0.00013 & 0.00013 & 0.01 & 0.912 \\ \text { GROUND*ASYM } & 2 & 0.00722 & 0.00722 & 0.00361 & 0.35 & 0.707 \\ \text { Error } & 317 & 3.29254 & 3.29254 & 0.01039 & & \\ \text { Total } & 335 & 4.99252 & & & & \\ \text { S }=0.101915 & \mathrm{R}-\mathrm{Sq}=34.05 \% & \mathrm{R}-\mathrm{Sq}(\operatorname{adj})=30.31 \% & \end{array}$

Analysis of Variance for TOTEX S1, using Adjusted SS for Tests

$\begin{array}{lrrrrrr}\text { Source } & \text { DF } & \text { Seq SS } & \text { Adj SS } & \text { Adj MS } & \text { F } & \text { P } \\ \text { Subject } & 13 & 0.0590909 & 0.0590909 & 0.0045455 & 31.71 & 0.000 \\ \text { GROUND } & 2 & 0.0028461 & 0.0028461 & 0.0014231 & 9.93 & 0.000 \\ \text { ASYM } & 1 & 0.0002214 & 0.0002214 & 0.0002214 & 1.54 & 0.215 \\ \text { GROUND*ASYM } & 2 & 0.0006536 & 0.0006536 & 0.0003268 & 2.28 & 0.104 \\ \text { Error } & 317 & 0.0454377 & 0.0454377 & 0.0001433 & & \\ \text { Total } & 335 & 0.1082498 & & & & \\ \text { S }=0.0119723 & \text { R-Sq }=58.03 \% & R-S q(\text { adj) }=55.64 \% & & \end{array}$


Analysis of Variance for TOTEX AP S1, using Adjusted SS for Tests

$\begin{array}{lrrrrrr}\text { Source } & \text { DF } & \text { Seq SS } & \text { Adj SS } & \text { Adj MS } & \text { F } & P \\ \text { Subject } & 13 & 0.0199473 & 0.0199473 & 0.0015344 & 26.52 & 0.000 \\ \text { GROUND } & 2 & 0.0009726 & 0.0009726 & 0.0004863 & 8.41 & 0.000 \\ \text { ASYM } & 1 & 0.0008097 & 0.0008097 & 0.0008097 & 14.00 & 0.000 \\ \text { GROUND*ASYM } & 2 & 0.0002513 & 0.0002513 & 0.0001257 & 2.17 & 0.116 \\ \text { Error } & 317 & 0.0183396 & 0.0183396 & 0.0000579 & & \\ \text { Total } & 335 & 0.0403206 & & & & \\ \text { S }=0.00760615 & \text { R-Sq }=54.52 \% & \text { R-Sq(adj) }=51.93 \%\end{array}$

Analysis of Variance for TOTEX ML S1, using Adjusted SS for Tests

$\begin{array}{lrrrrrr}\text { Source } & \text { DF } & \text { Seq SS } & \text { Adj SS } & \text { Adj MS } & F & P \\ \text { Subject } & 13 & 0.0355204 & 0.0355204 & 0.0027323 & 26.45 & 0.000 \\ \text { GROUND } & 2 & 0.0014626 & 0.0014626 & 0.0007313 & 7.08 & 0.001 \\ \text { ASYM } & 1 & 0.0000335 & 0.0000335 & 0.0000335 & 0.32 & 0.570 \\ \text { GROUND*ASYM } & 2 & 0.0002888 & 0.0002888 & 0.0001444 & 1.40 & 0.249 \\ \text { Error } & 317 & 0.0327500 & 0.0327500 & 0.0001033 & & \\ \text { Total } & 335 & 0.0700553 & & & & \end{array}$

$\mathrm{S}=0.0101643 \quad \mathrm{R}-\mathrm{Sq}=53.25 \% \mathrm{R}-\mathrm{Sq}(\operatorname{adj})=50.60 \%$

Analysis of Variance for MVELO S1, using Adjusted SS for Tests

$\begin{array}{lrrrrrr}\text { Source } & \text { DF } & \text { Seq SS } & \text { Adj SS } & \text { Adj MS } & \text { F } & P \\ \text { Subject } & 13 & 0.0030441 & 0.0030441 & 0.0002342 & 33.21 & 0.000 \\ \text { GROUND } & 2 & 0.0001366 & 0.0001366 & 0.0000683 & 9.69 & 0.000 \\ \text { ASYM } & 1 & 0.0000108 & 0.0000108 & 0.0000108 & 1.53 & 0.217 \\ \text { GROUND*ASYM } & 2 & 0.0000323 & 0.0000323 & 0.0000162 & 2.29 & 0.103 \\ \text { Error } & 317 & 0.0022350 & 0.0022350 & 0.0000071 & & \\ \text { Total } & 335 & 0.0054588 & & & & \\ \text { S }=0.00265528 & \text { R-Sq }=59.06 \% & R-S q(\text { adj) }=56.73 \% & \end{array}$

Analysis of Variance for MVELO AP S1, using Adjusted SS for Tests

$\begin{array}{lrrrrrr}\text { Source } & \text { DF } & \text { Seq SS } & \text { Adj SS } & \text { Adj MS } & \text { F } & \text { P } \\ \text { Subject } & 13 & 0.0010198 & 0.0010198 & 0.0000784 & 27.62 & 0.000 \\ \text { GROUND } & 2 & 0.0000467 & 0.0000467 & 0.0000234 & 8.23 & 0.000 \\ \text { ASYM } & 1 & 0.0000394 & 0.0000394 & 0.0000394 & 13.88 & 0.000 \\ \text { GROUND*ASYM } & 2 & 0.0000124 & 0.0000124 & 0.0000062 & 2.18 & 0.114 \\ \text { Error } & 317 & 0.0009003 & 0.0009003 & 0.0000028 & & \\ \text { Total } & 335 & 0.0020186 & & & & \\ \text { S }=0.00168526 & \text { R-Sq }=55.40 \% & R-S q(\text { adj })=52.87 \% & & \end{array}$

Analysis of Variance for MVELO ML S1, using Adjusted SS for Tests

$\begin{array}{lrrrrrr}\text { Source } & \text { DF } & \text { Seq SS } & \text { Adj SS } & \text { Adj MS } & \text { F } & \text { P } \\ \text { Subject } & 13 & 0.0018220 & 0.0018220 & 0.0001402 & 27.54 & 0.000 \\ \text { GROUND } & 2 & 0.0000703 & 0.0000703 & 0.0000351 & 6.91 & 0.001 \\ \text { ASYM } & 1 & 0.0000016 & 0.0000016 & 0.0000016 & 0.32 & 0.572 \\ \text { GROUND*ASYM } & 2 & 0.0000143 & 0.0000143 & 0.0000072 & 1.41 & 0.246 \\ \text { Error } & 317 & 0.0016130 & 0.0016130 & 0.0000051 & & \\ \text { Total } & 335 & 0.0035212 & & & & \end{array}$




\begin{tabular}{|c|c|c|c|c|c|c|c|}
\hline \multicolumn{2}{|c|}{$S=0.00225573$} & $\mathrm{R}-\mathrm{Sq}=54.19$ & \multicolumn{3}{|c|}{$R-S q(\operatorname{adj})=51.59 \%$} & \multirow{2}{*}{\multicolumn{2}{|c|}{ for Test: }} \\
\hline Analysis of & Varia & nce for ARE? & A CC S1, us & ising Ad & justed & & \\
\hline Source & $\mathrm{DF}$ & Seq SS & Adj $S S$ & Adj MS & $\mathrm{F}$ & $\mathrm{P}$ & $P$ \\
\hline Subject & 13 & 105.8541 & 105.8541 & 8.1426 & 11.98 & 0.000 & \\
\hline GROUND & 2 & 0.3979 & 0.3979 & 0.1989 & 0.29 & 0.746 & \\
\hline ASYM & 1 & 2.1324 & 2.1324 & 2.1324 & 3.14 & 0.077 & \\
\hline GROUND *ASYM & 2 & 0.3897 & 0.3897 & 0.1948 & 0.29 & 0.751 & \\
\hline Error & 317 & 215.4223 & 215.4223 & 0.6796 & & & \\
\hline Total & 335 & 324.1964 & & & & & \\
\hline$S=0.824358$ & \multicolumn{2}{|c|}{$\mathrm{R}-\mathrm{Sq}=33.55 \%$} & \multicolumn{3}{|c|}{$R-S q(\operatorname{adj})=29.78 \%$} & & \\
\hline Analysis of & \multicolumn{2}{|c|}{ Variance for $A R E$} & $\mathrm{~A}$ CE S1, us & Ising Ad & justed & SS for & $r$ Tests \\
\hline Source & $\mathrm{DF}$ & Seq SS & $\operatorname{Adj} S S$ & Adj MS & $\mathrm{F}$ & $\mathrm{P}$ & $P$ \\
\hline Subject & 13 & 69.5664 & 69.5664 & 5.3513 & 13.73 & 0.000 & \\
\hline GROUND & 2 & 0.0985 & 0.0985 & 0.0492 & 0.13 & 0.881 & \\
\hline ASYM & 1 & 3.6332 & 3.6332 & 3.6332 & 9.32 & 0.002 & \\
\hline GROUND *ASYM & 2 & 0.3109 & 0.3109 & 0.1554 & 0.40 & 0.671 & \\
\hline Error & 317 & 123.5731 & 123.5731 & 0.3898 & & & \\
\hline Total & 335 & 197.1820 & & & & & \\
\hline$S=0.624356$ & & $\mathrm{Sq}=37.33 \%$ & $\mathrm{R}-\mathrm{Sq}(\mathrm{adj}$ & $(j)=33$ & $.77 \%$ & & \\
\hline Analysis of & Varia & nce for ARE? & A SW S1, us & Ising Ad & justed & SS for & $r$ Tests \\
\hline Source & $\mathrm{DF}$ & Seq SS & Adj $S S$ & Ad & j MS & F & $\mathrm{P}$ \\
\hline Subject & 13 & 0.0000216 & 0.0000216 & 0.000 & 0017 & 14.56 & 0.000 \\
\hline GROUND & 2 & 0.0000007 & 0.0000007 & 0.000 & 0003 & 3.03 & 0.050 \\
\hline ASYM & 1 & 0.0000003 & 0.0000003 & 0.000 & 0003 & 2.52 & 0.113 \\
\hline GROUND *A.SYM & 2 & 0.0000003 & 0.0000003 & 0.000 & 0001 & 1.17 & 0.312 \\
\hline Error & 317 & 0.0000363 & 0.0000363 & 0.000 & 0001 & & \\
\hline Total & 335 & 0.0000592 & & & & & \\
\hline$S=0.000338$ & 3198 & $\mathrm{R}-\mathrm{Sq}=38$ & $\mathrm{R}-\mathrm{Sq}$ & $(\operatorname{adj})=$ & 35.22 & & \\
\hline
\end{tabular}




\section{APPENDIX B. STATISTICAL MODEL ADEQUECY CHECKING}

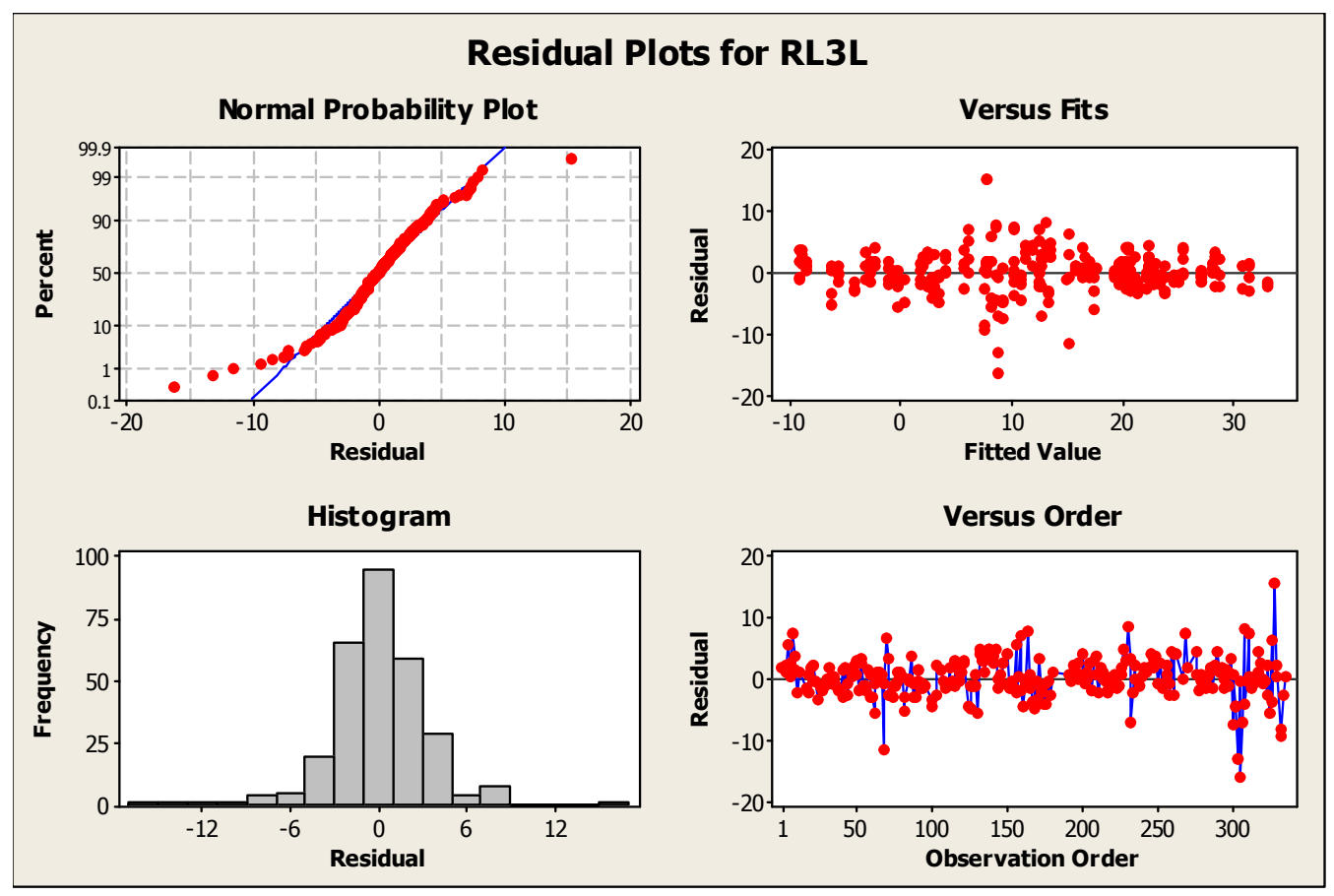

Figure 65: Residual plots for RL3L

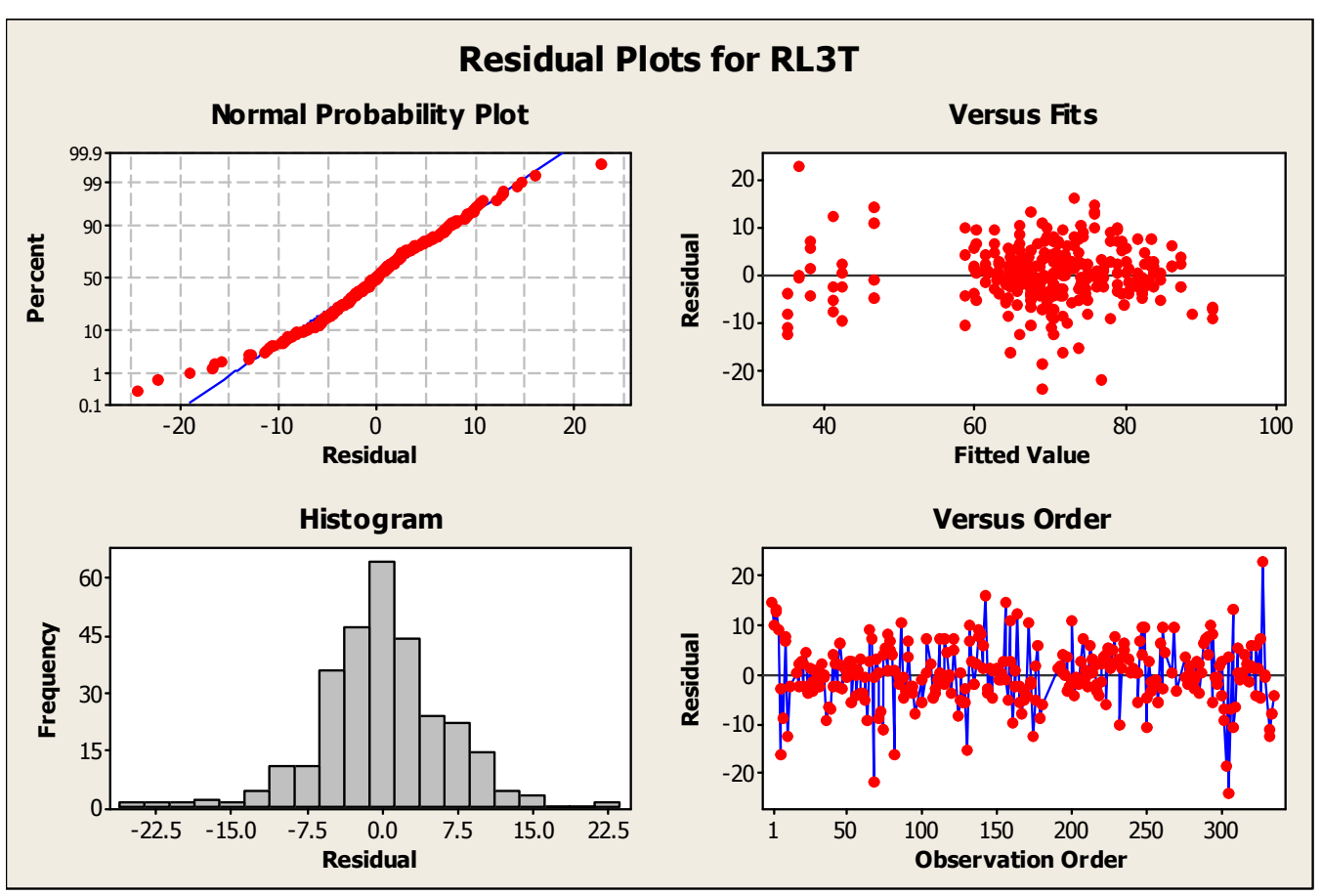

Figure 66: Residual plots for RL3T 


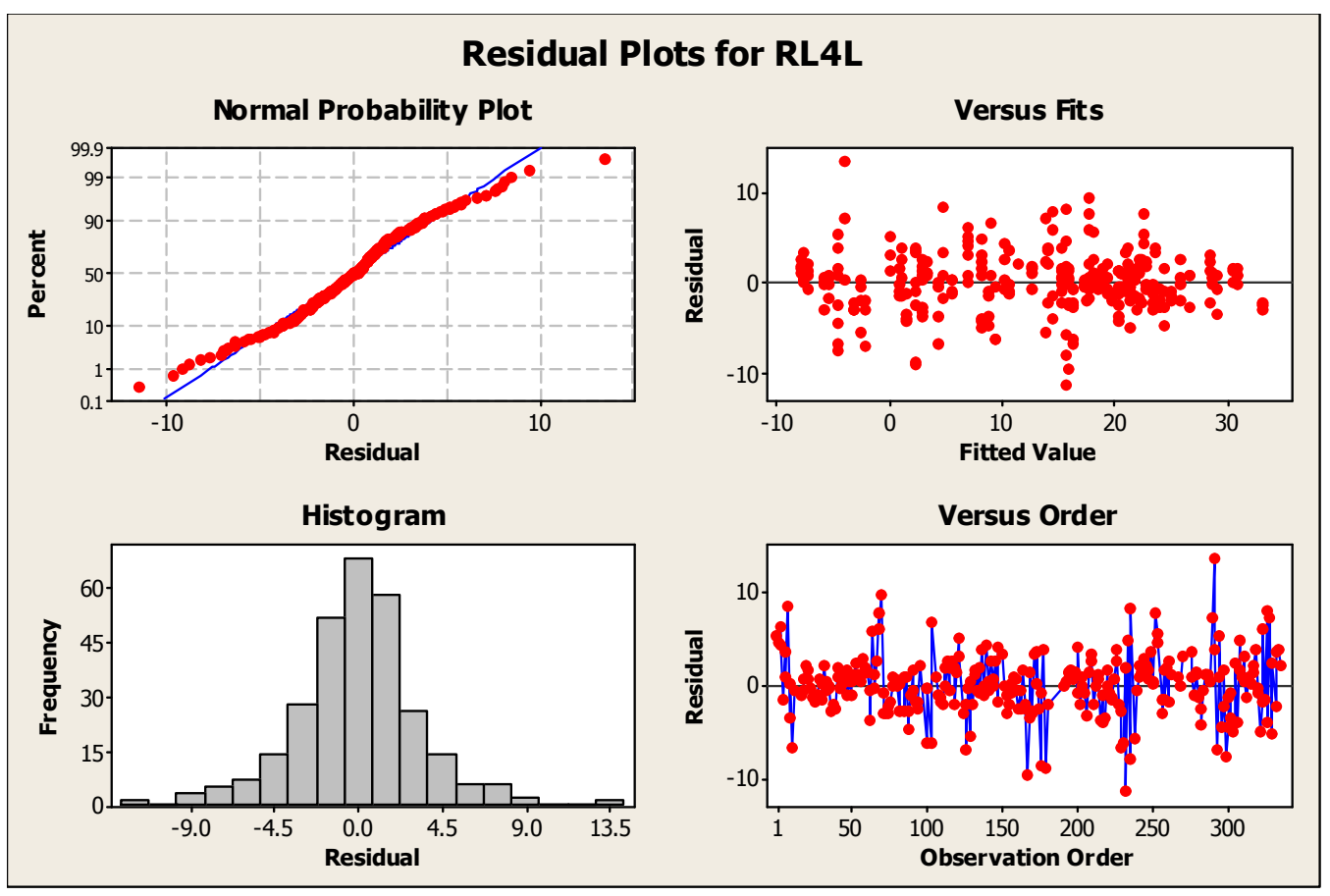

Figure 67: Residual plots for RL4L

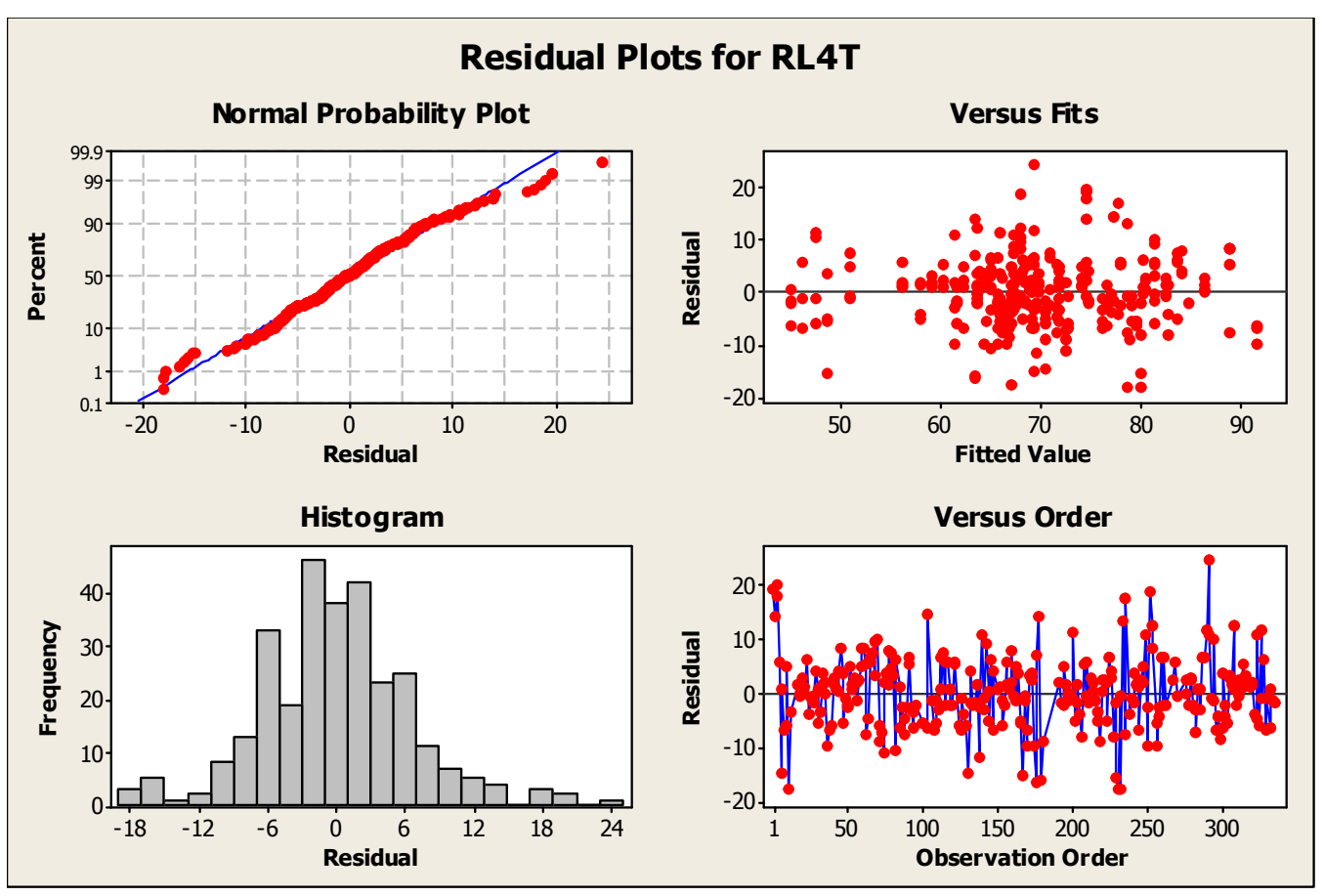

Figure 68: Residual plots for RL4T 


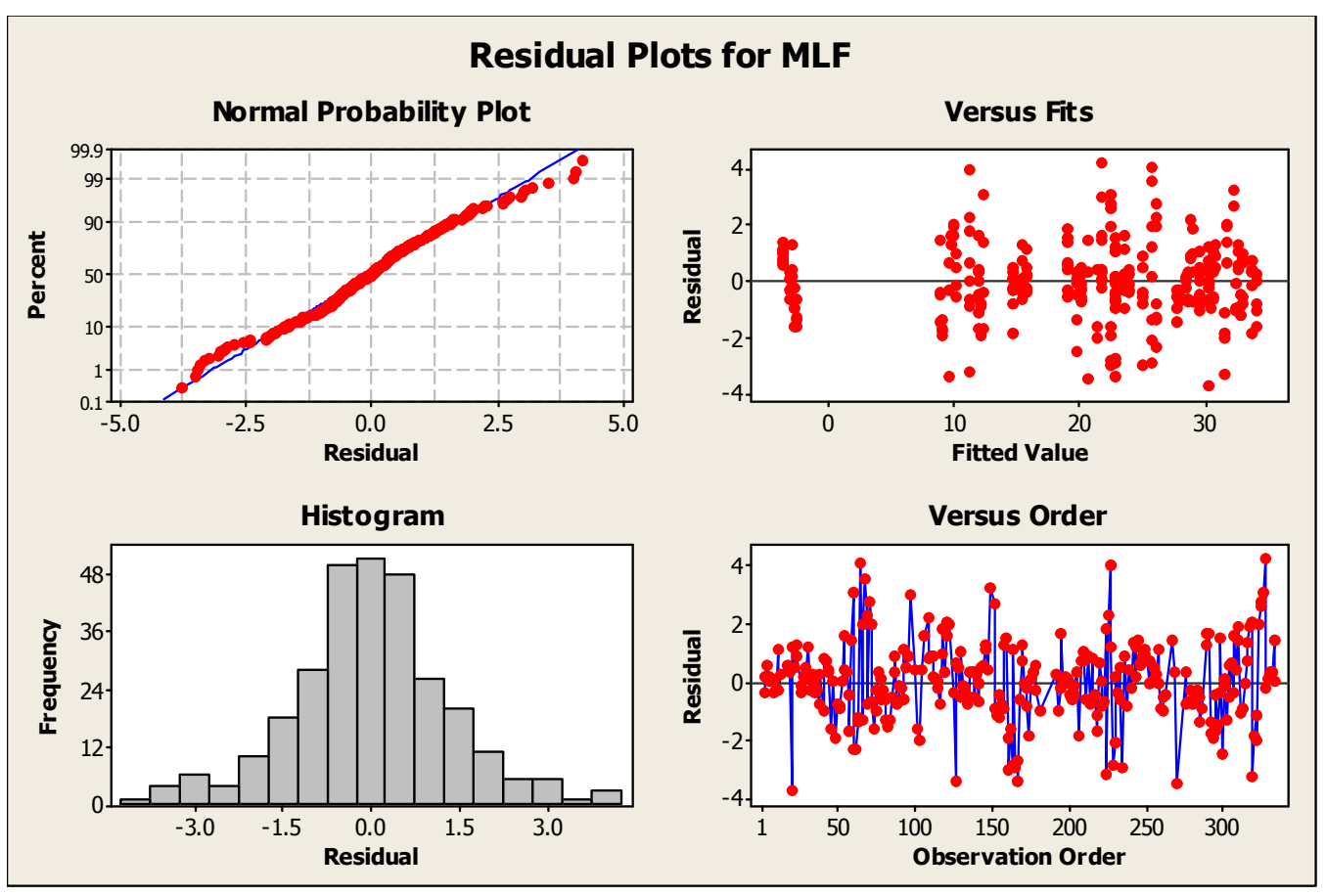

Figure 69: Residual plots for MLF

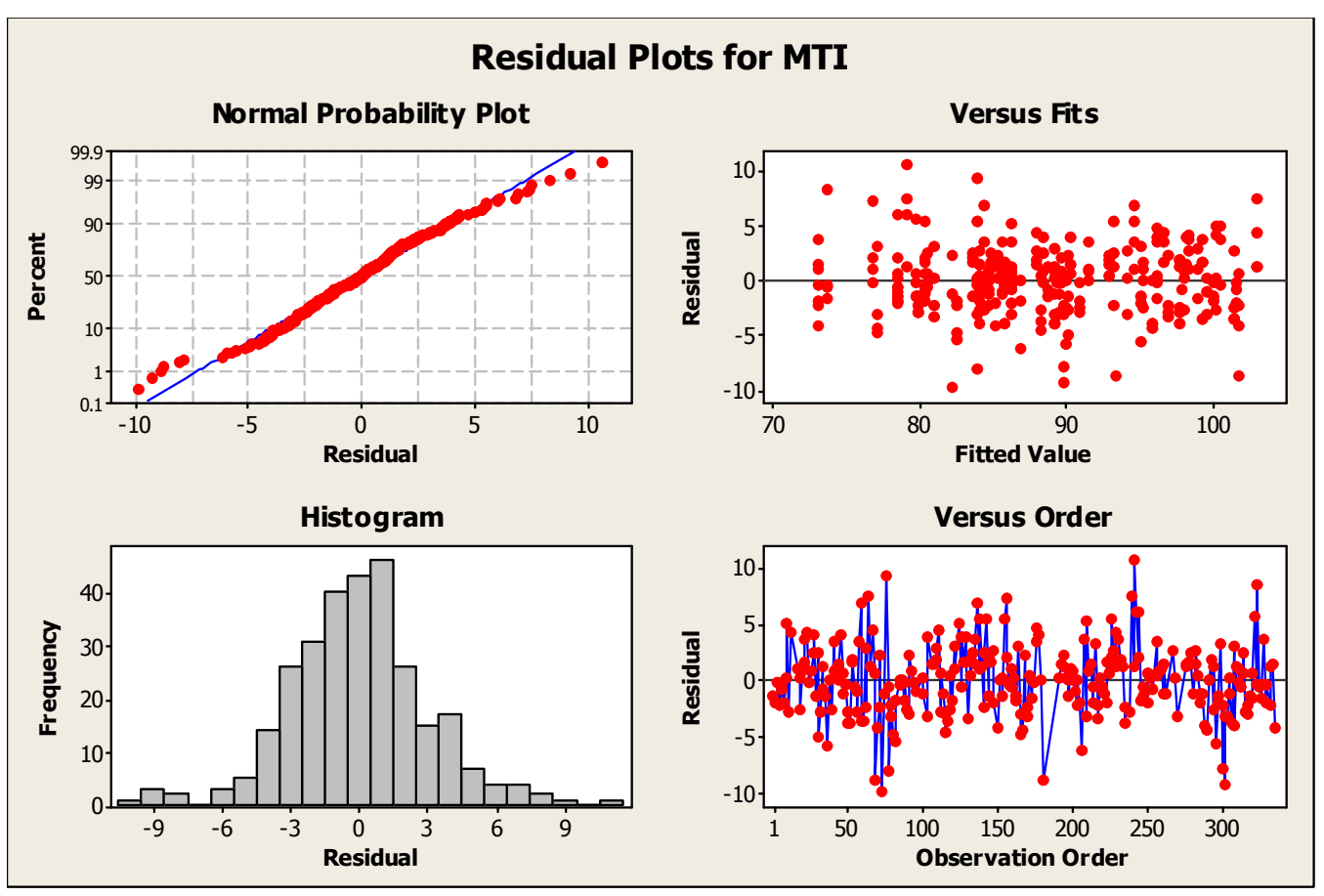

Figure 70: Residual plots for MTI 


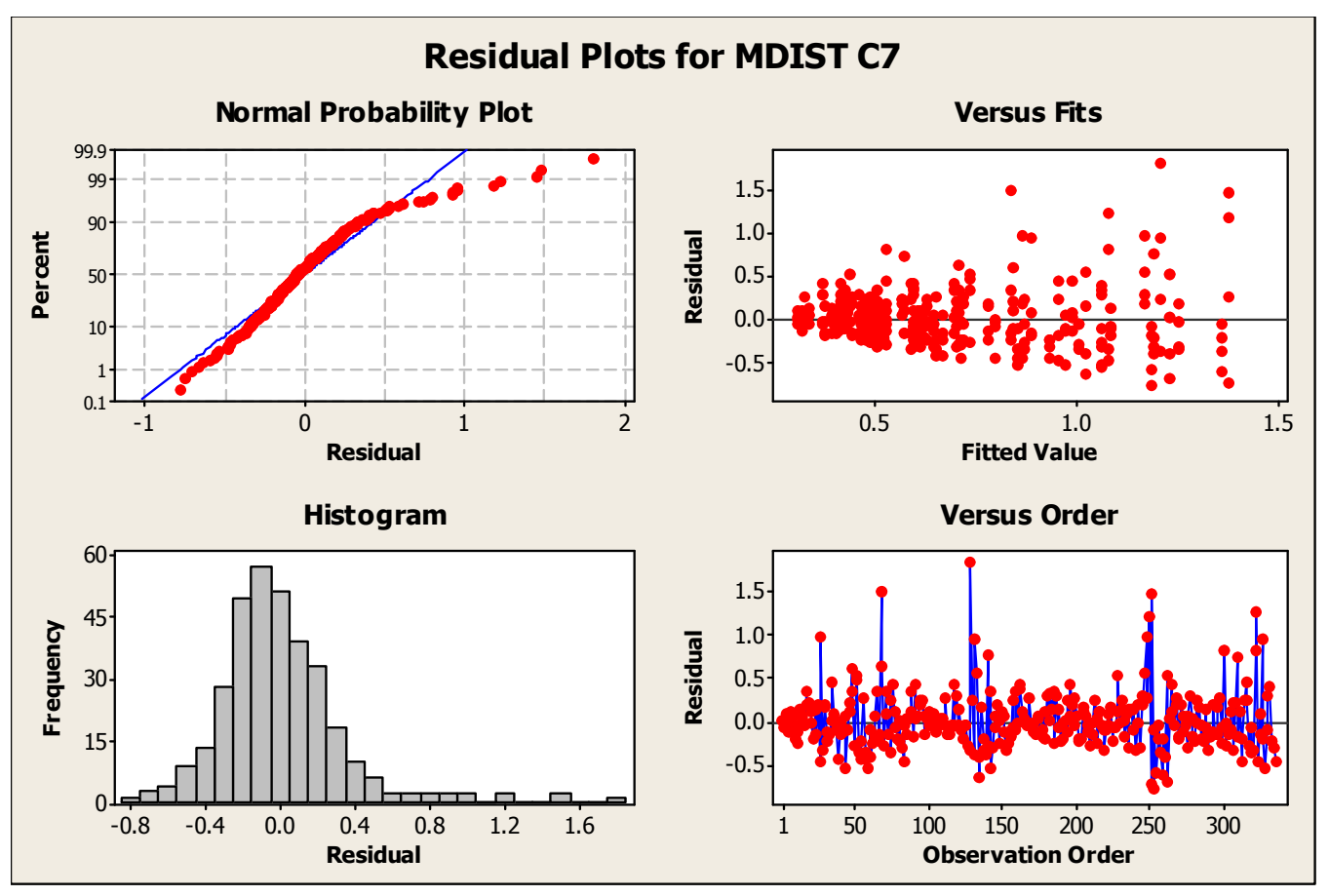

Figure 71: Residual plots for MDIST C7

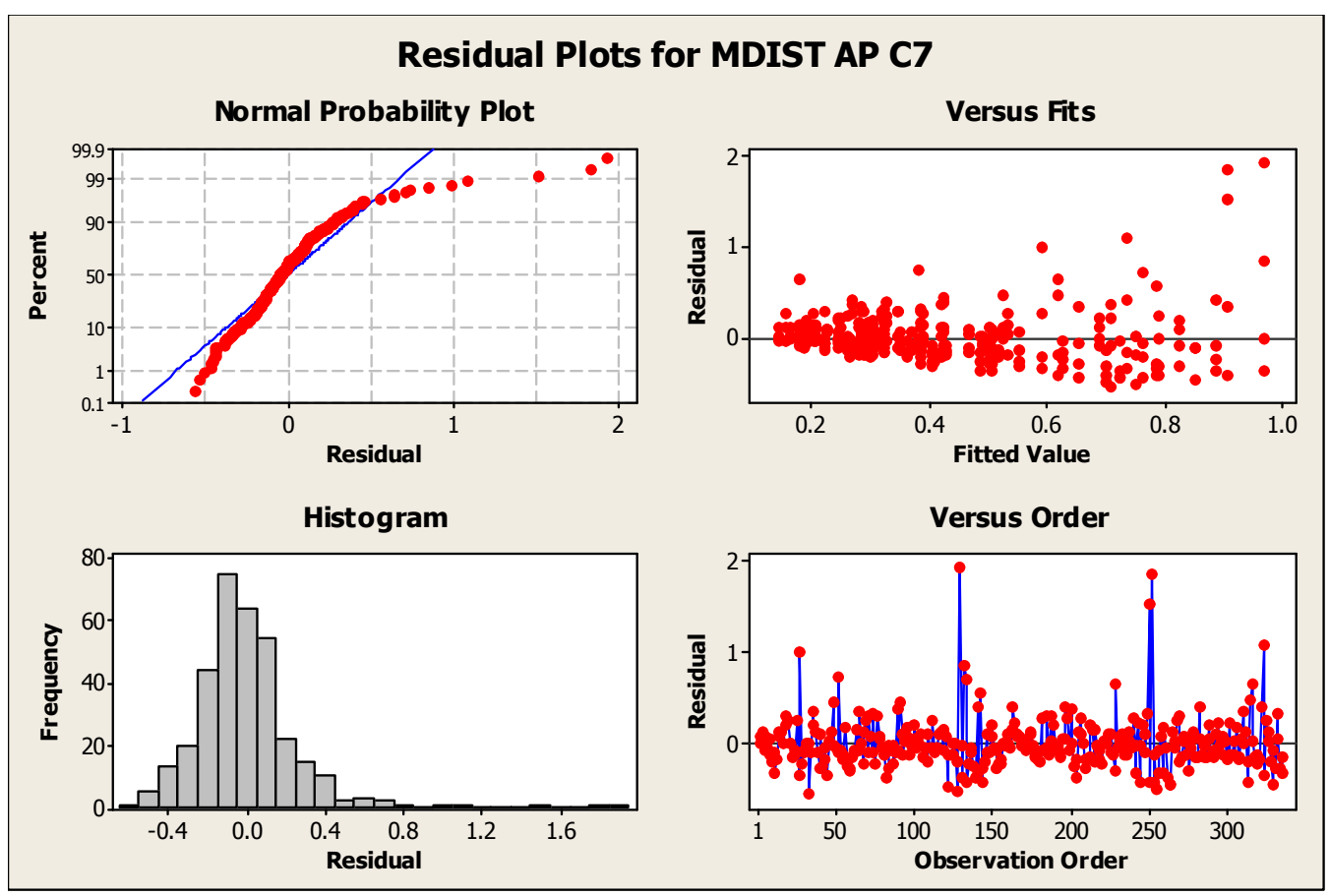

Figure 72: Residual plots for MDIST AP C7 


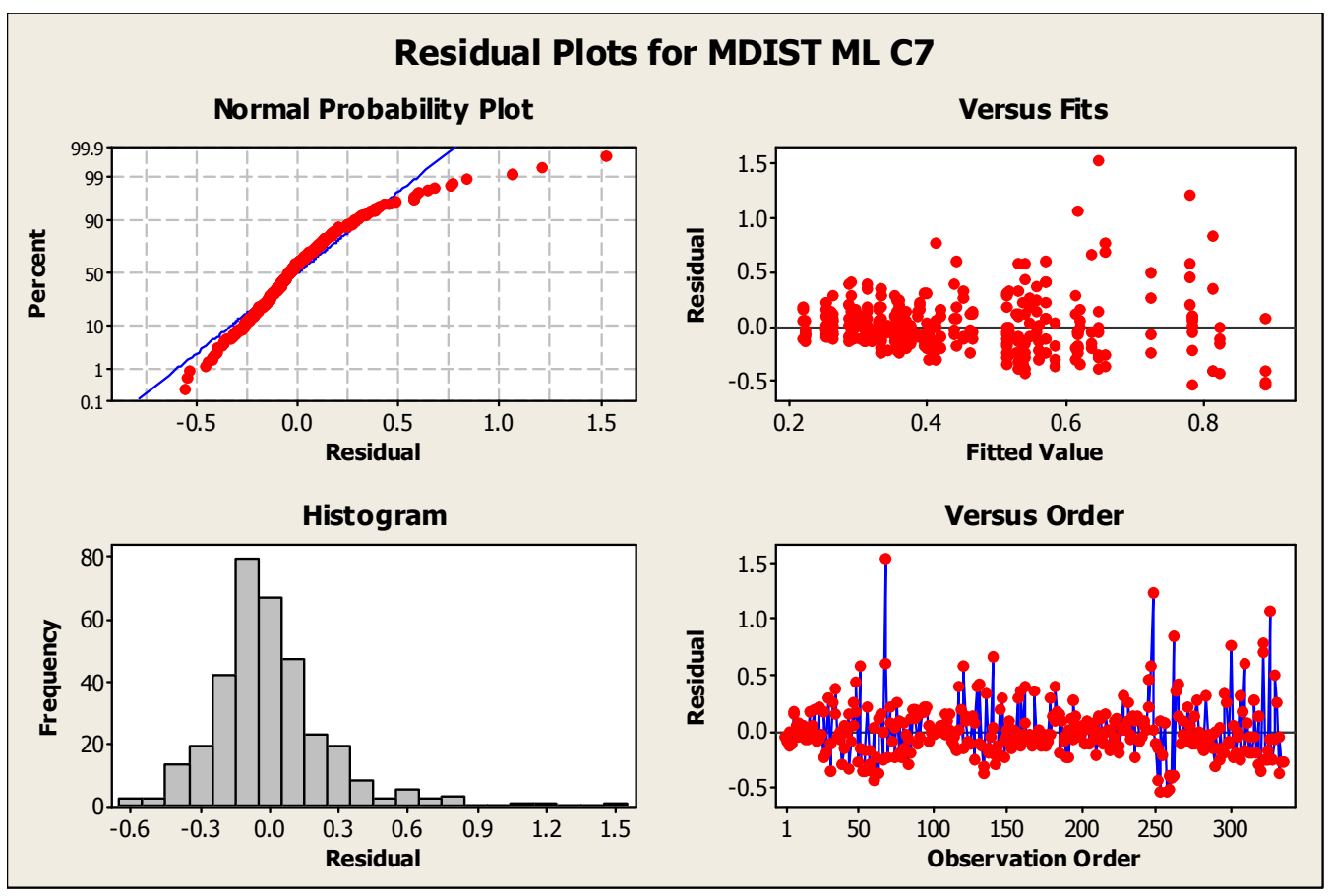

Figure 73: Residual plots for MDIST ML C7

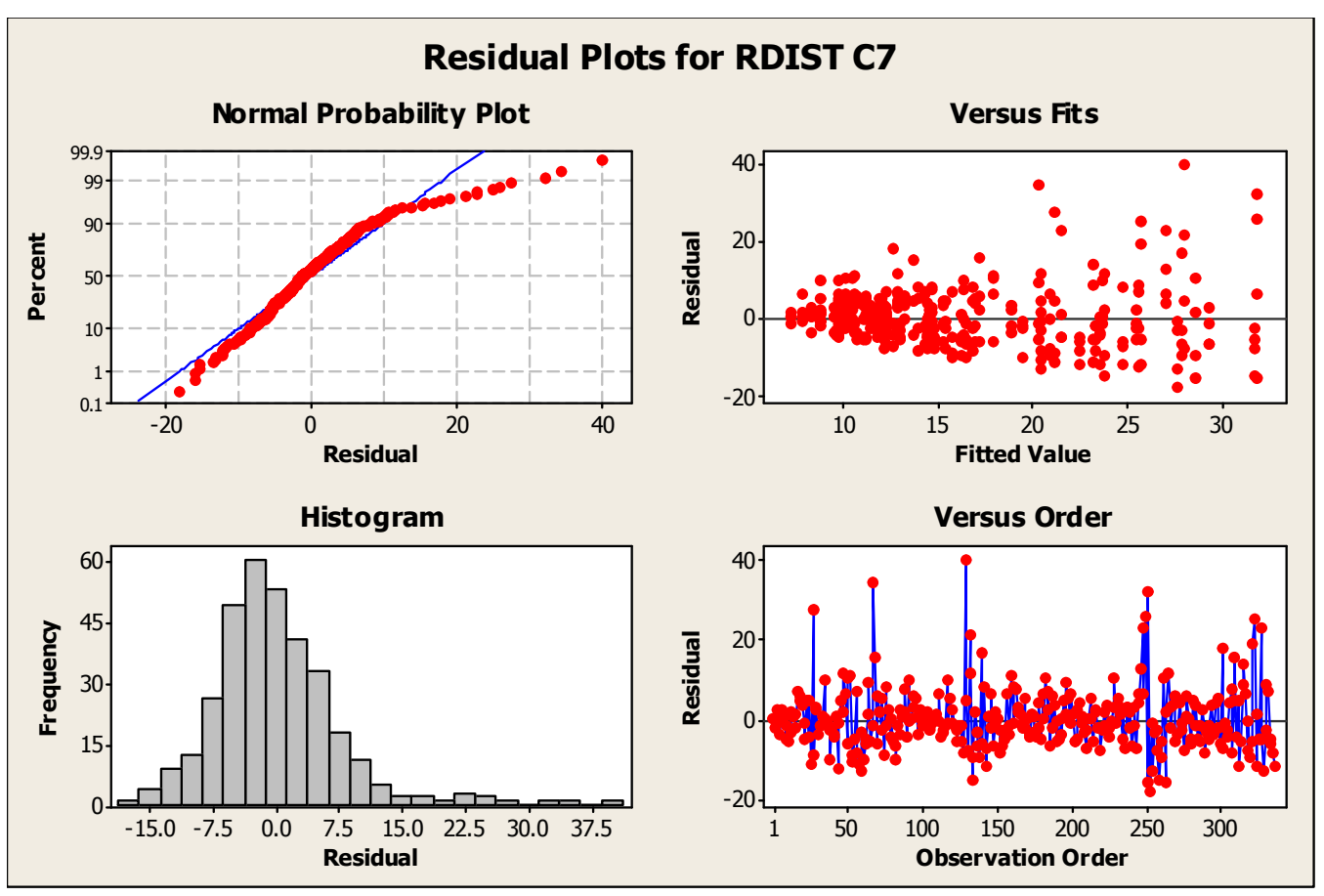

Figure 74: Residual plots for RDIST C7 


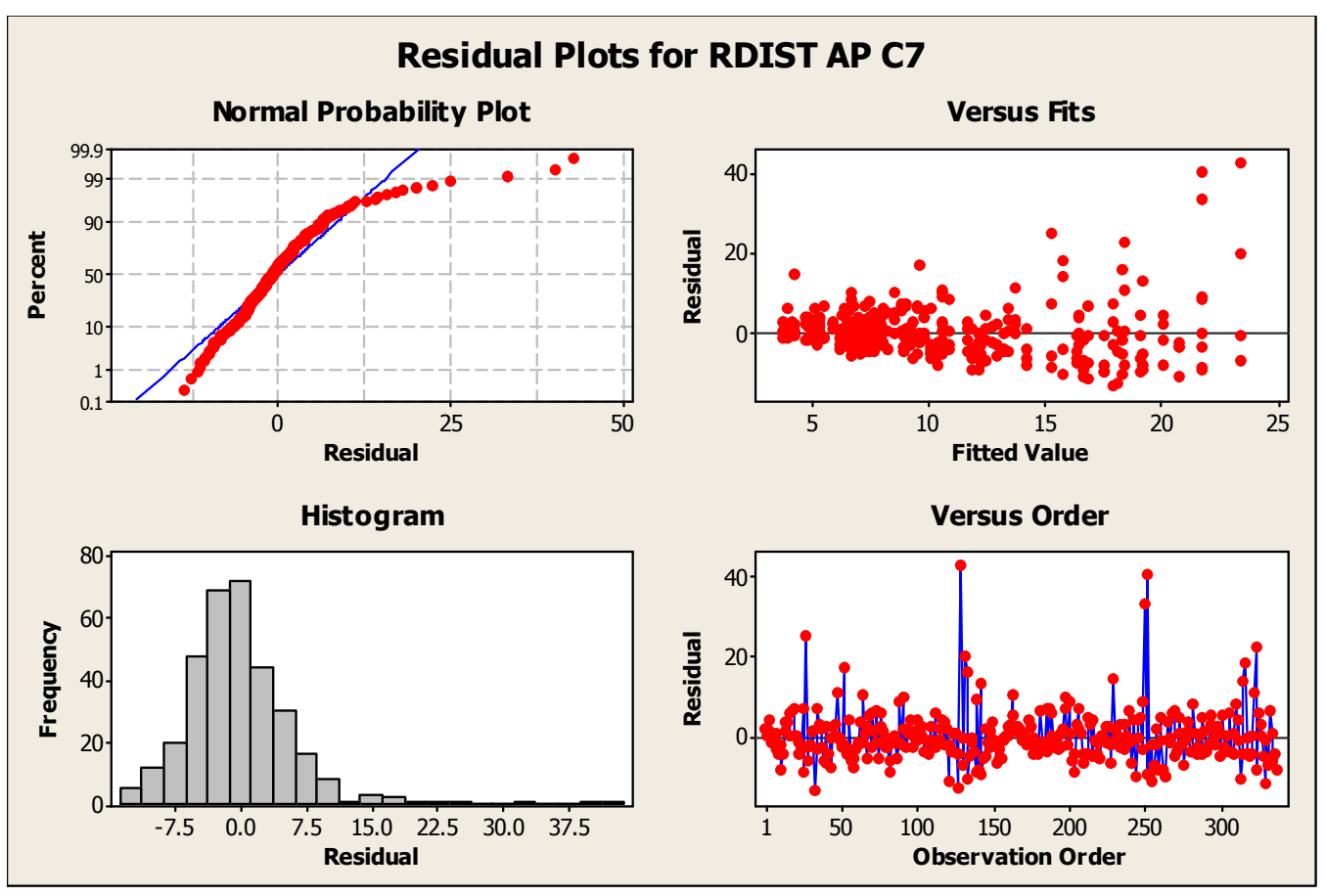

Figure 75: Residual plots for RDIST AP C7

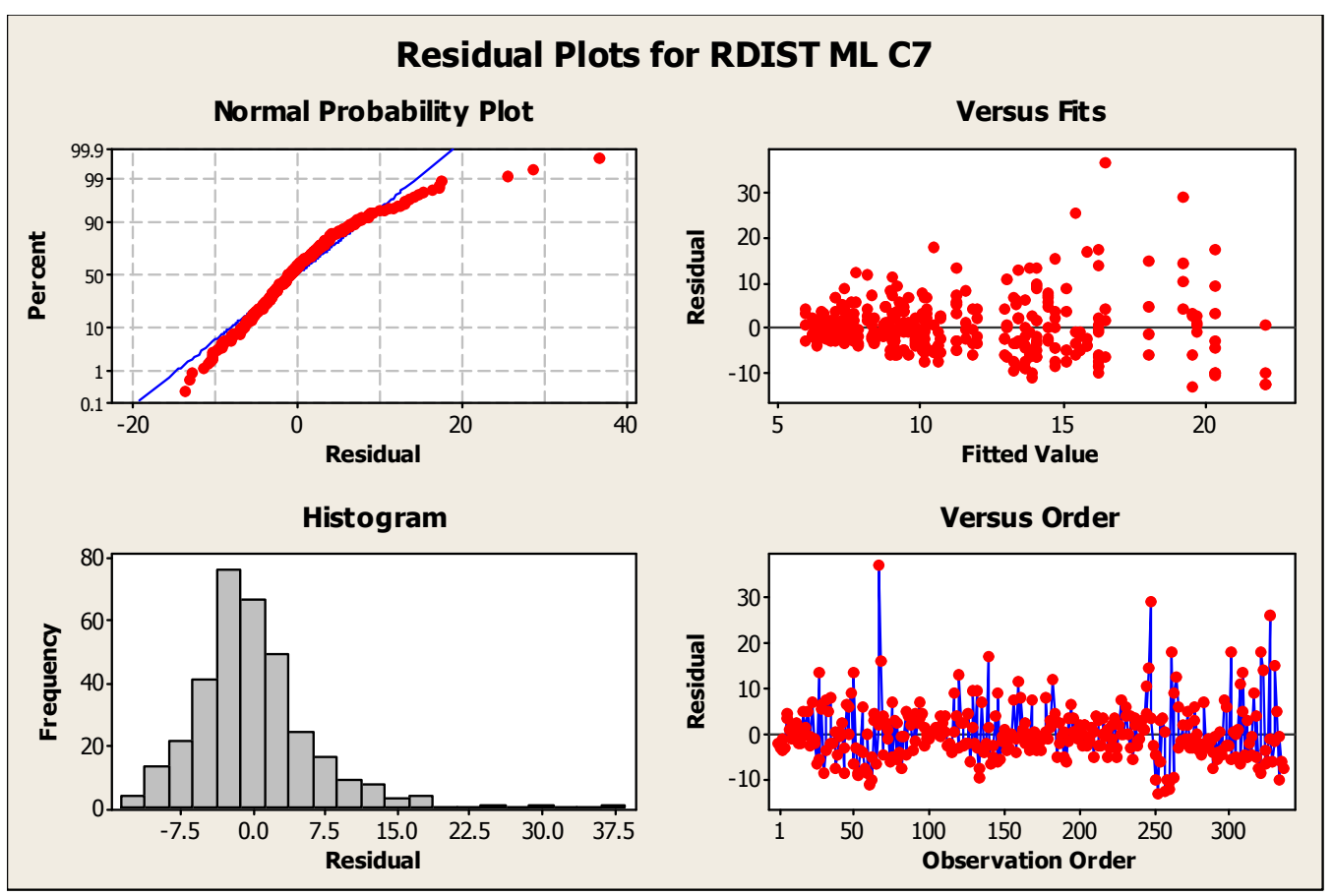

Figure 76: Residual plots for RDIST ML C7 


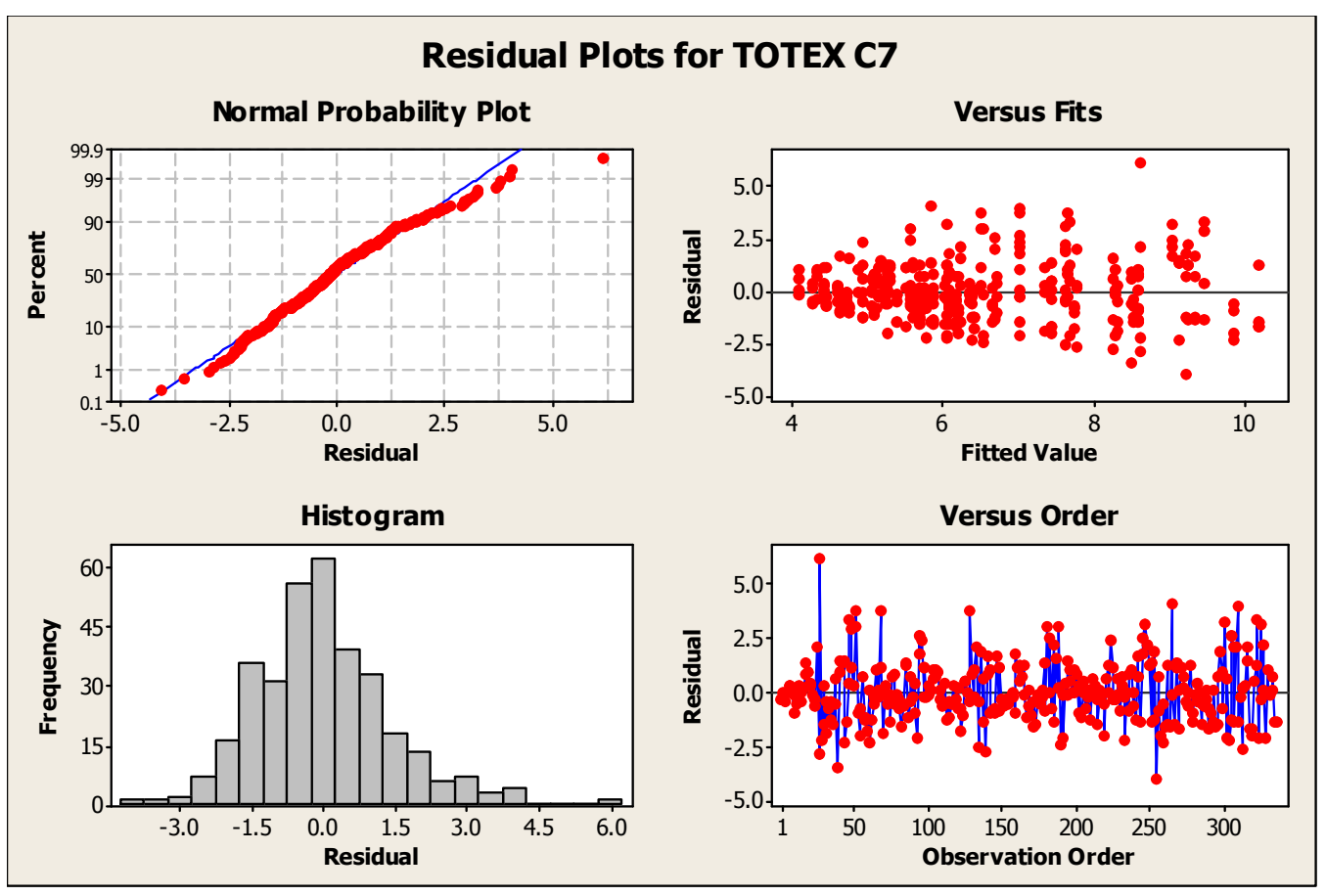

Figure 77: Residual plots for TOTEX C7

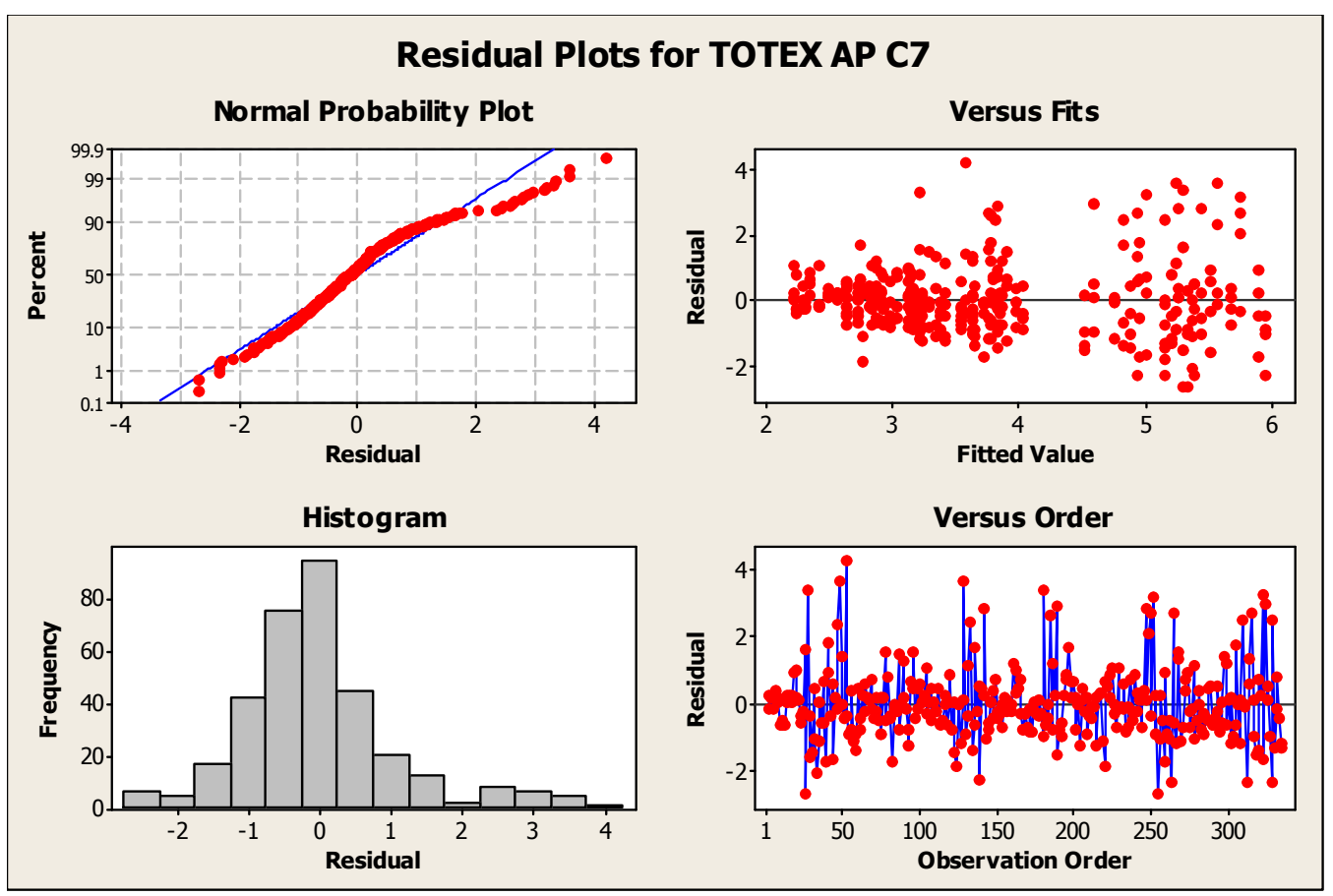

Figure 78: Residual plots for TOTEX AP C7 


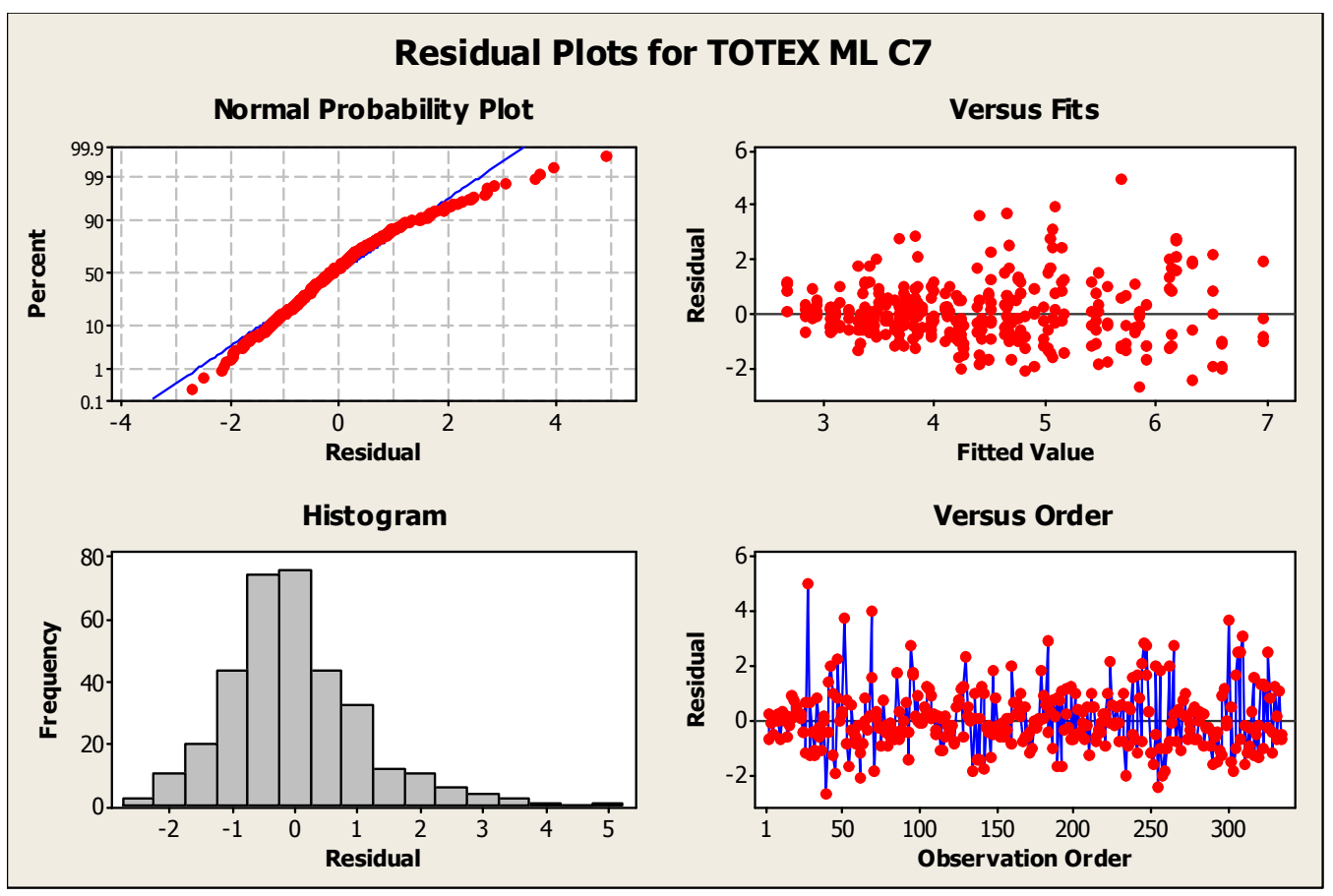

Figure 79: Residual plots for TOTEX ML C7

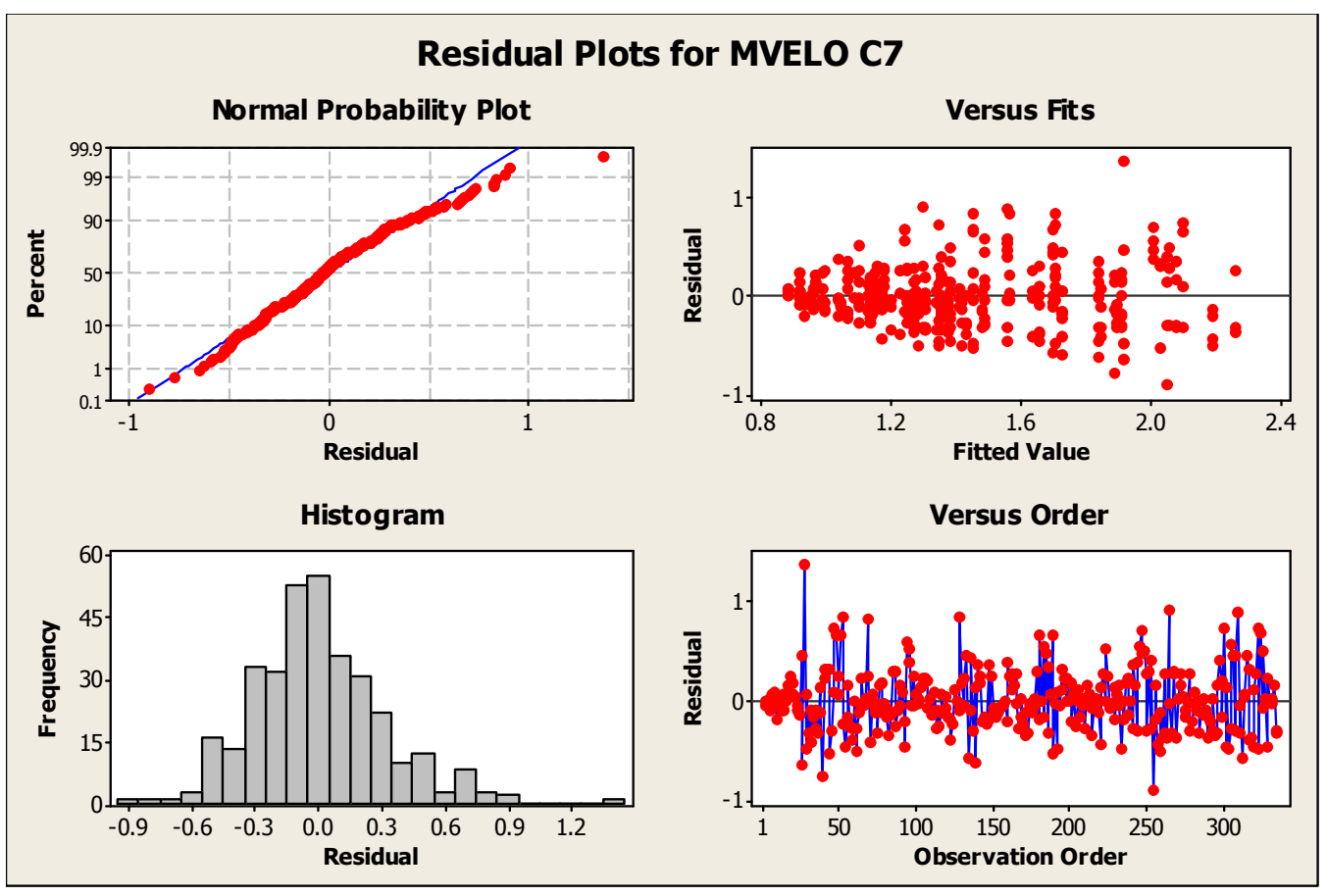

Figure 80: Residual plots for MVELO C7 


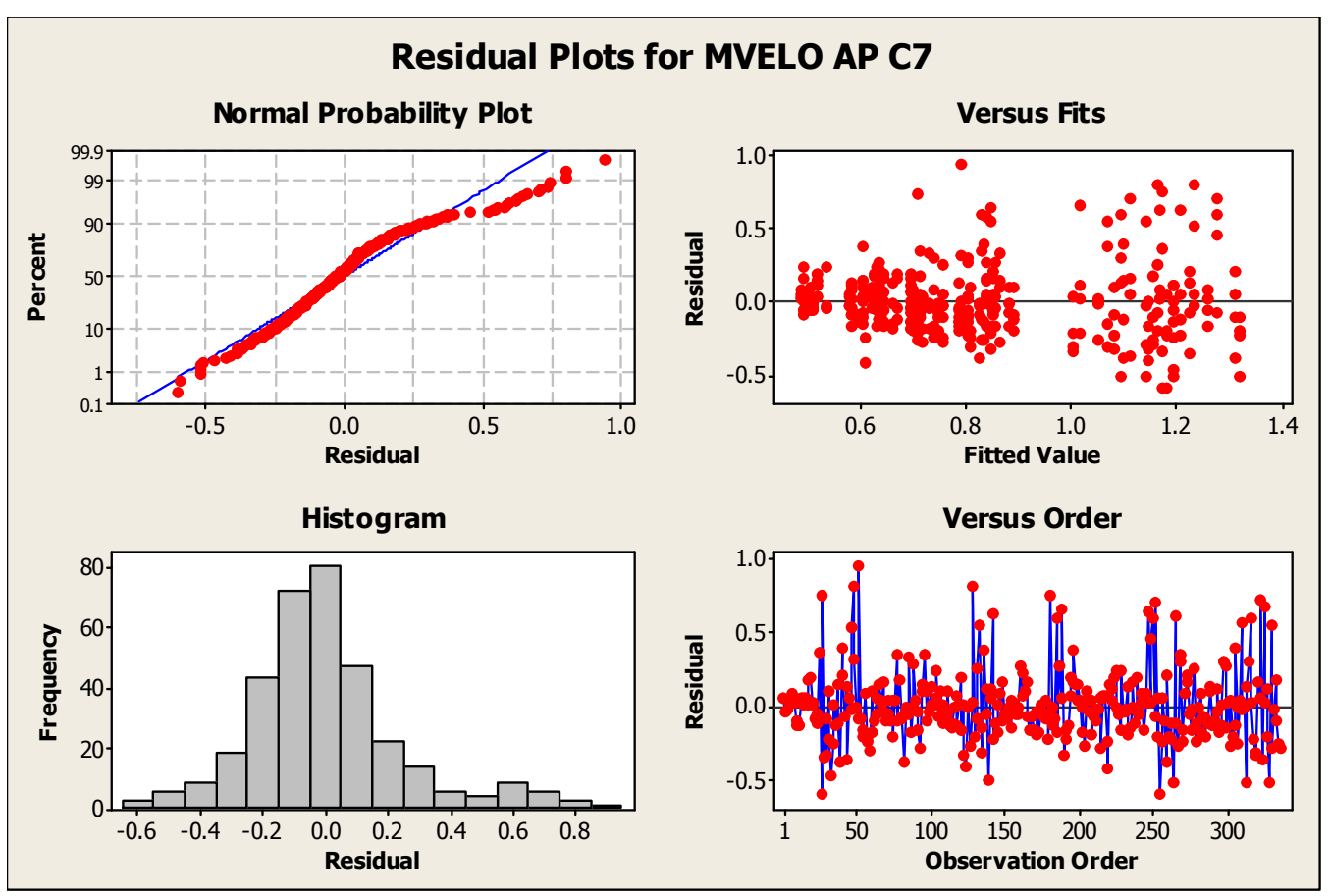

Figure 81: Residual plots for MVELO AP C7

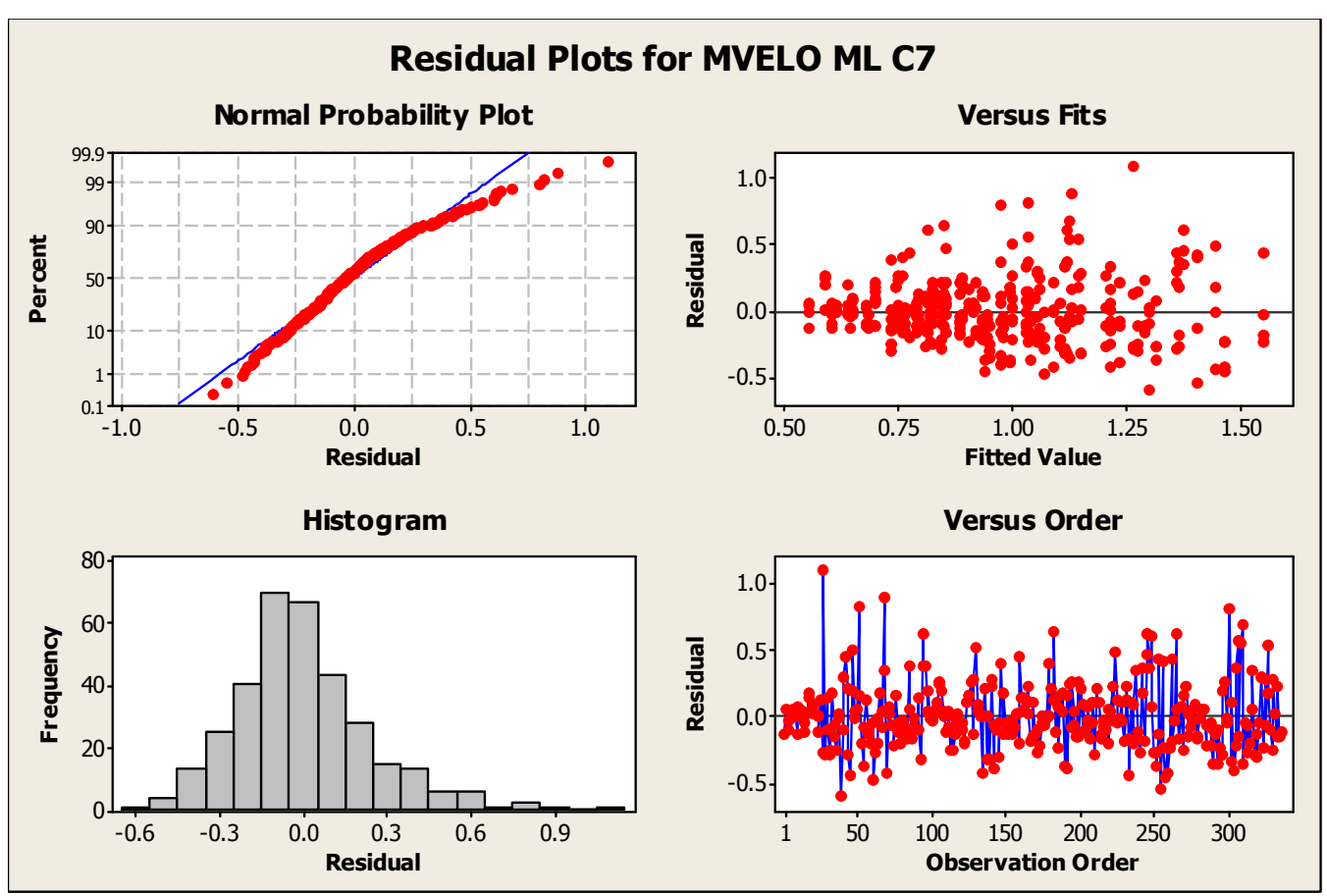

Figure 82: Residual plots for MVELO ML C7 


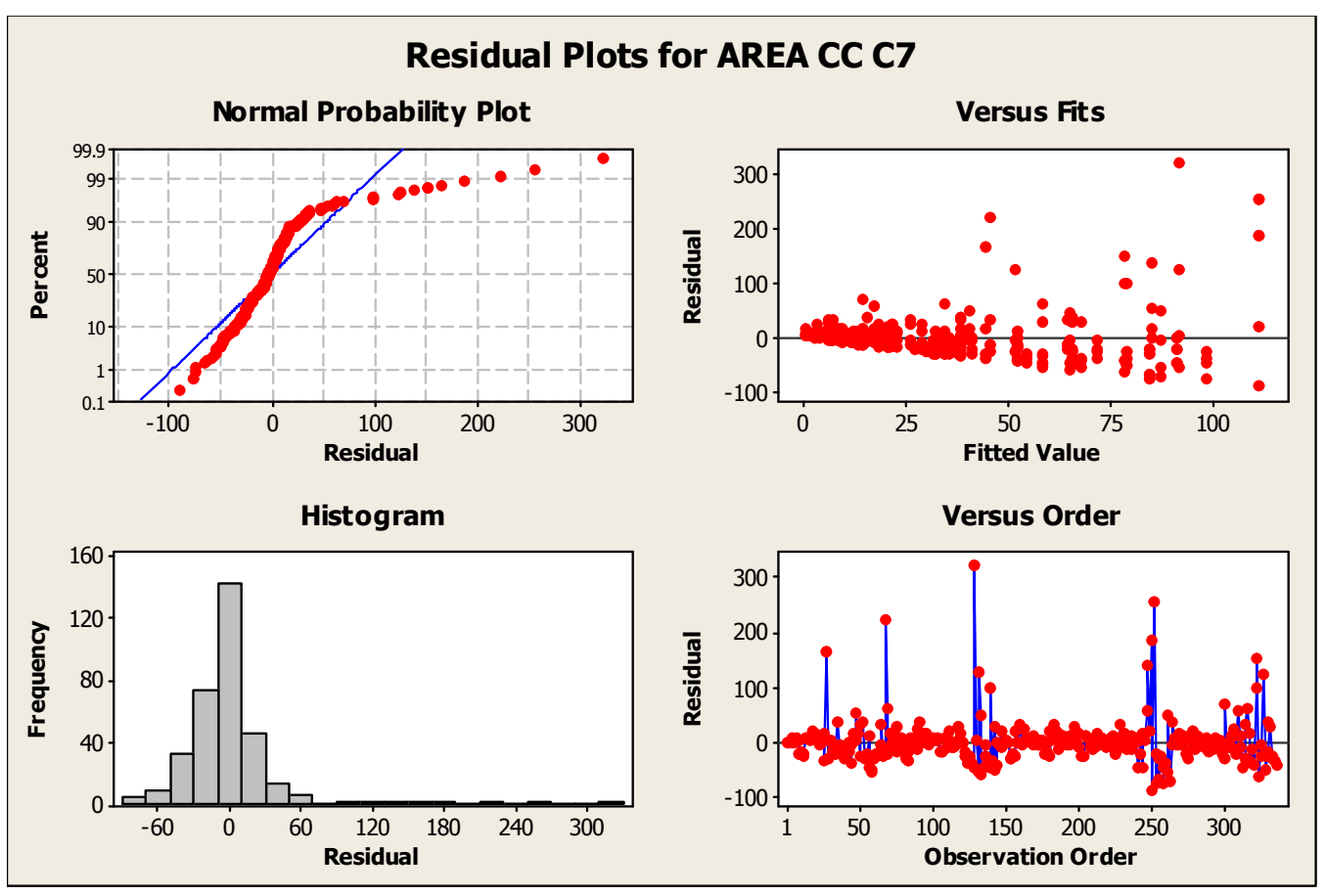

Figure 83: Residual plots for AREA CC C7

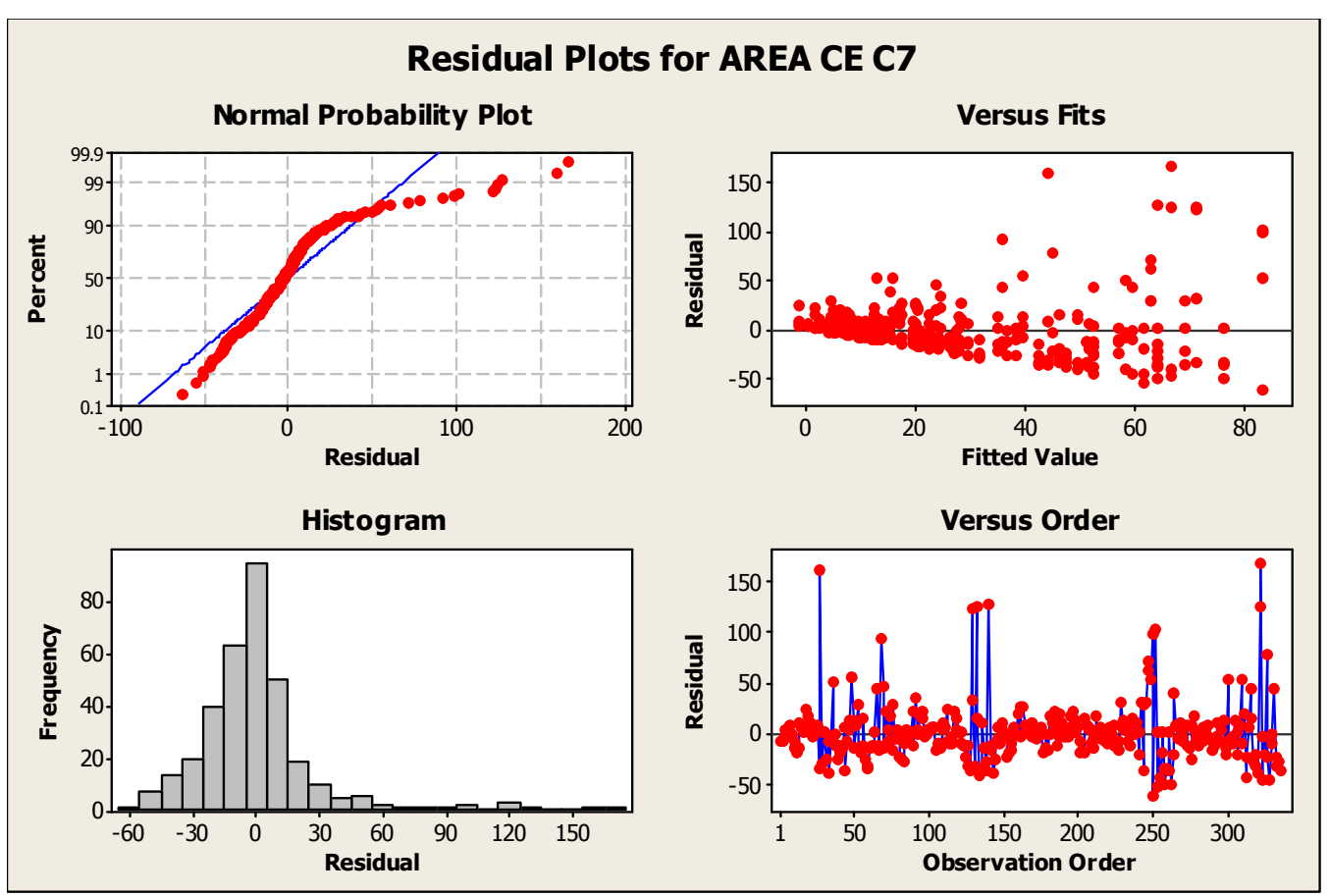

Figure 84: Residual plots for AREA CE C7 


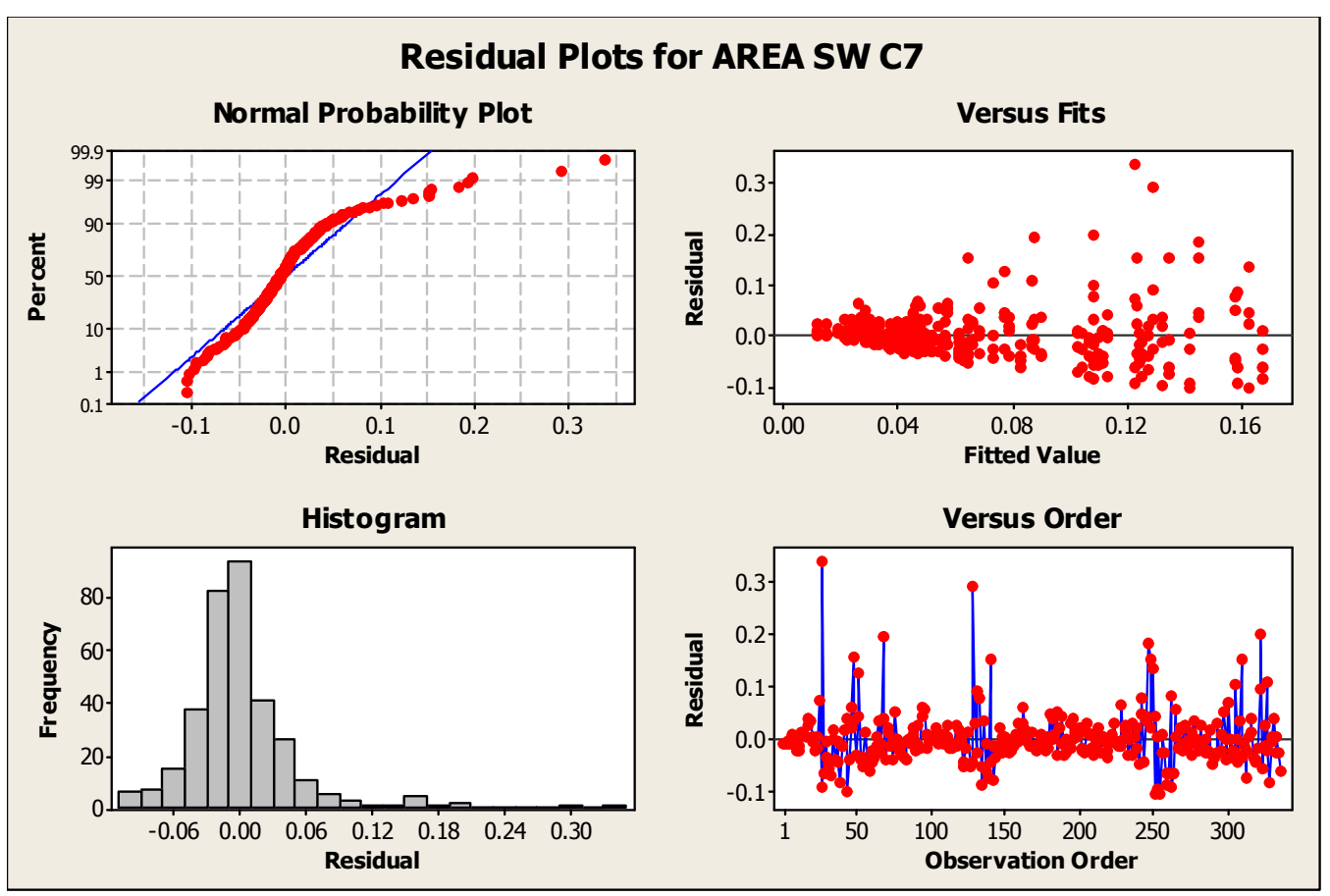

Figure 85: Residual plots for AREA SW C7

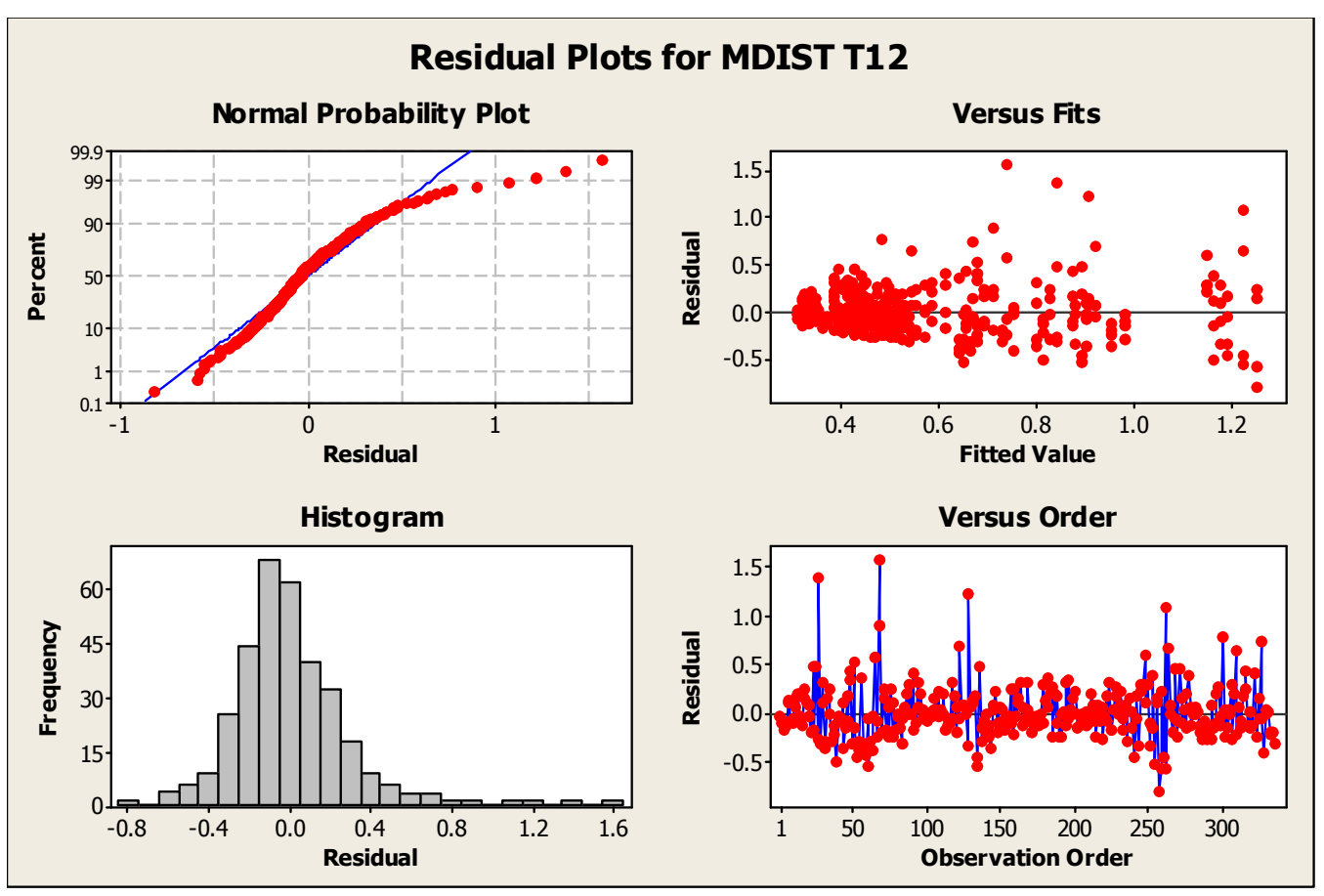

Figure 86: Residual plots for MDIST T12 


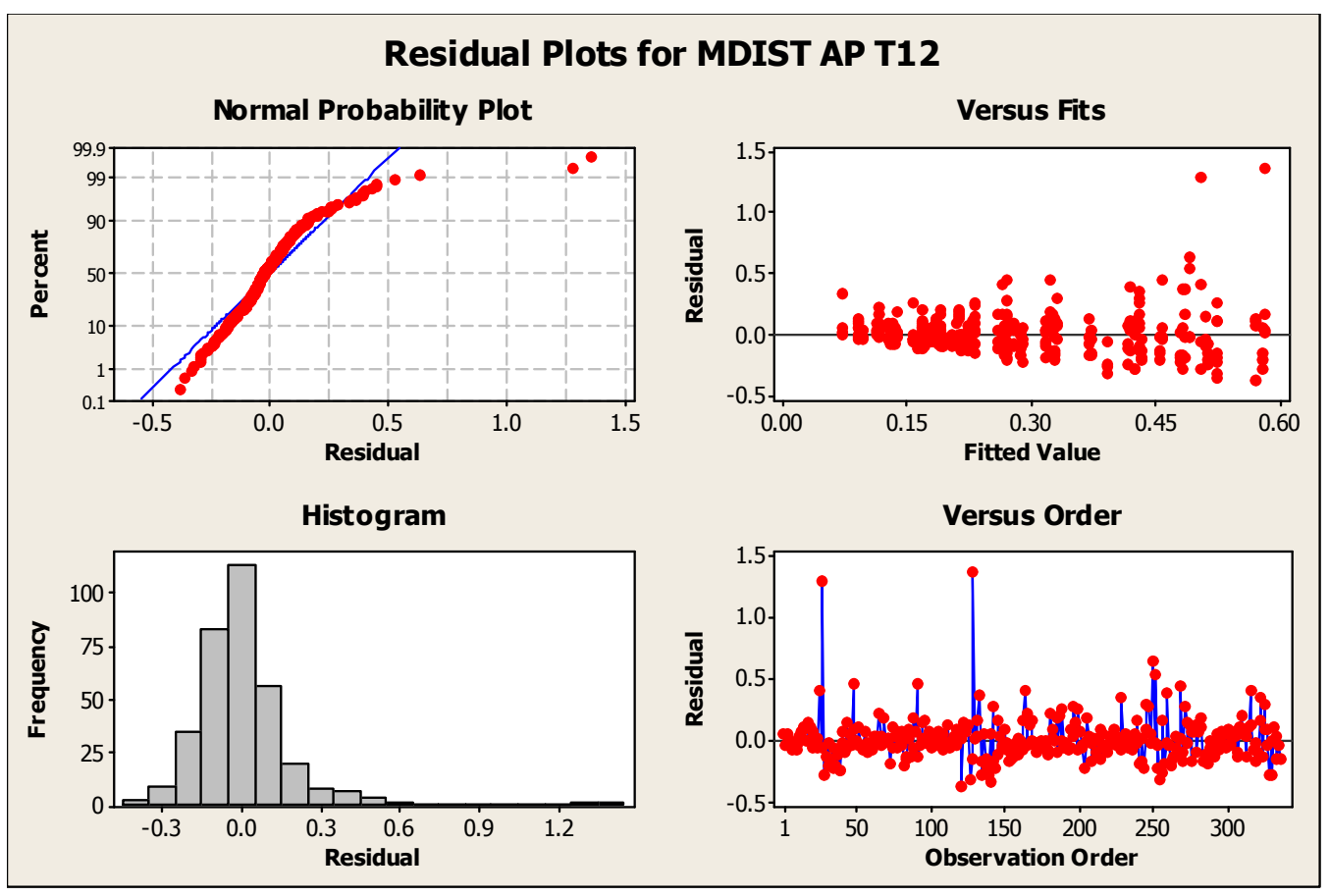

Figure 87: Residual plots for MDIST AP T12

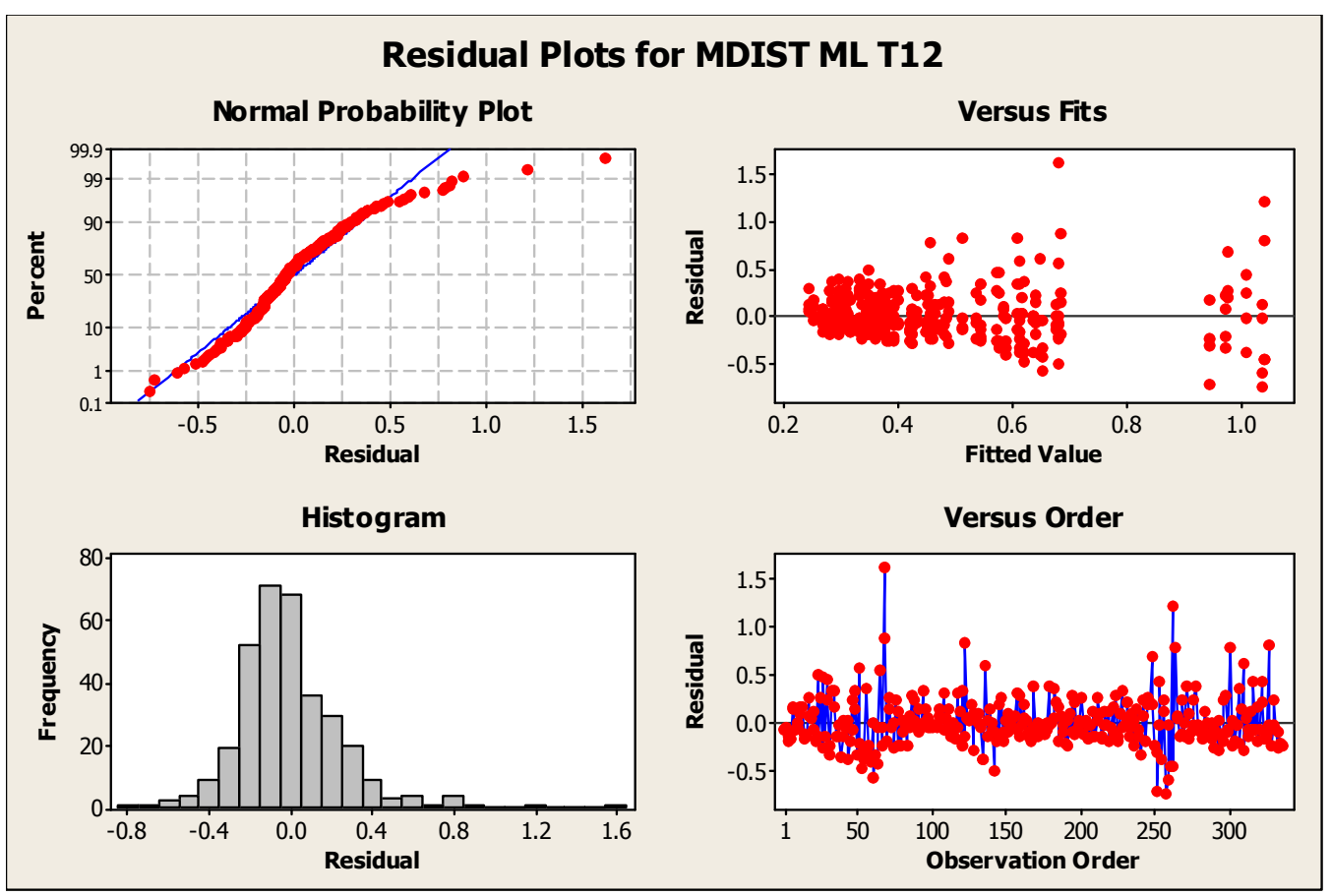

Figure 88: Residual plots for MDIST ML T12 


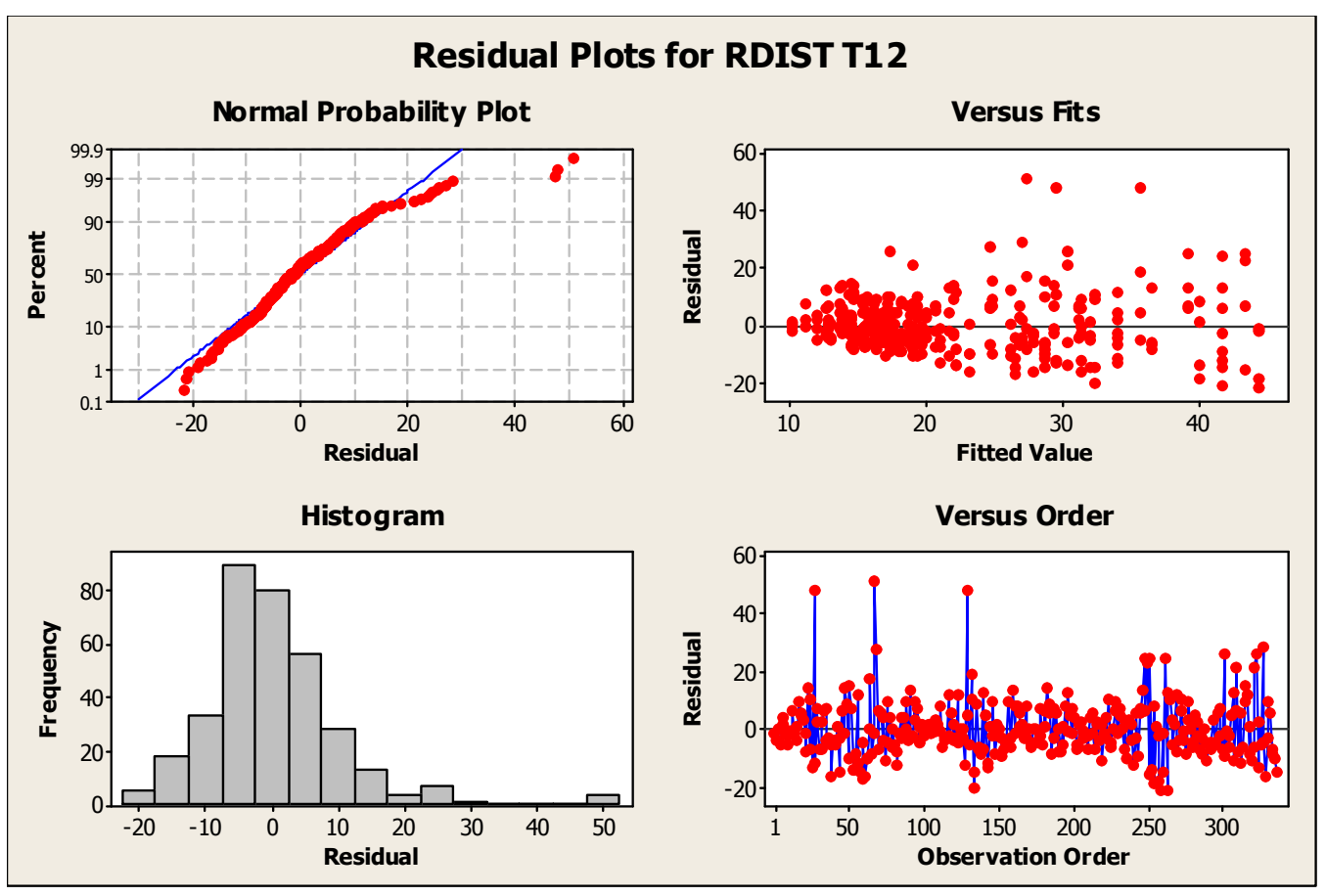

Figure 89: Residual plots for RDIST T12

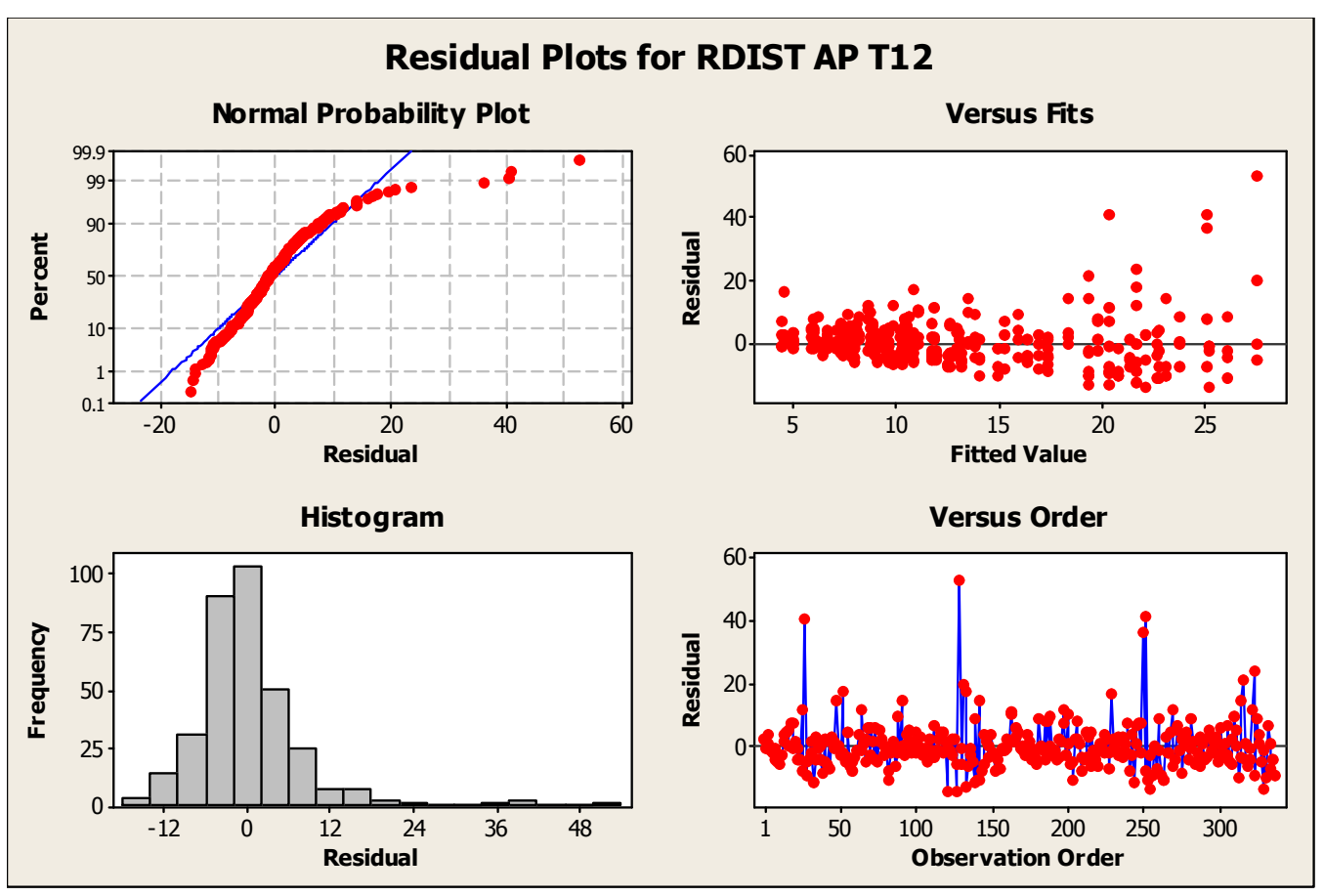

Figure 90: Residual plots for RDIST AP T12 


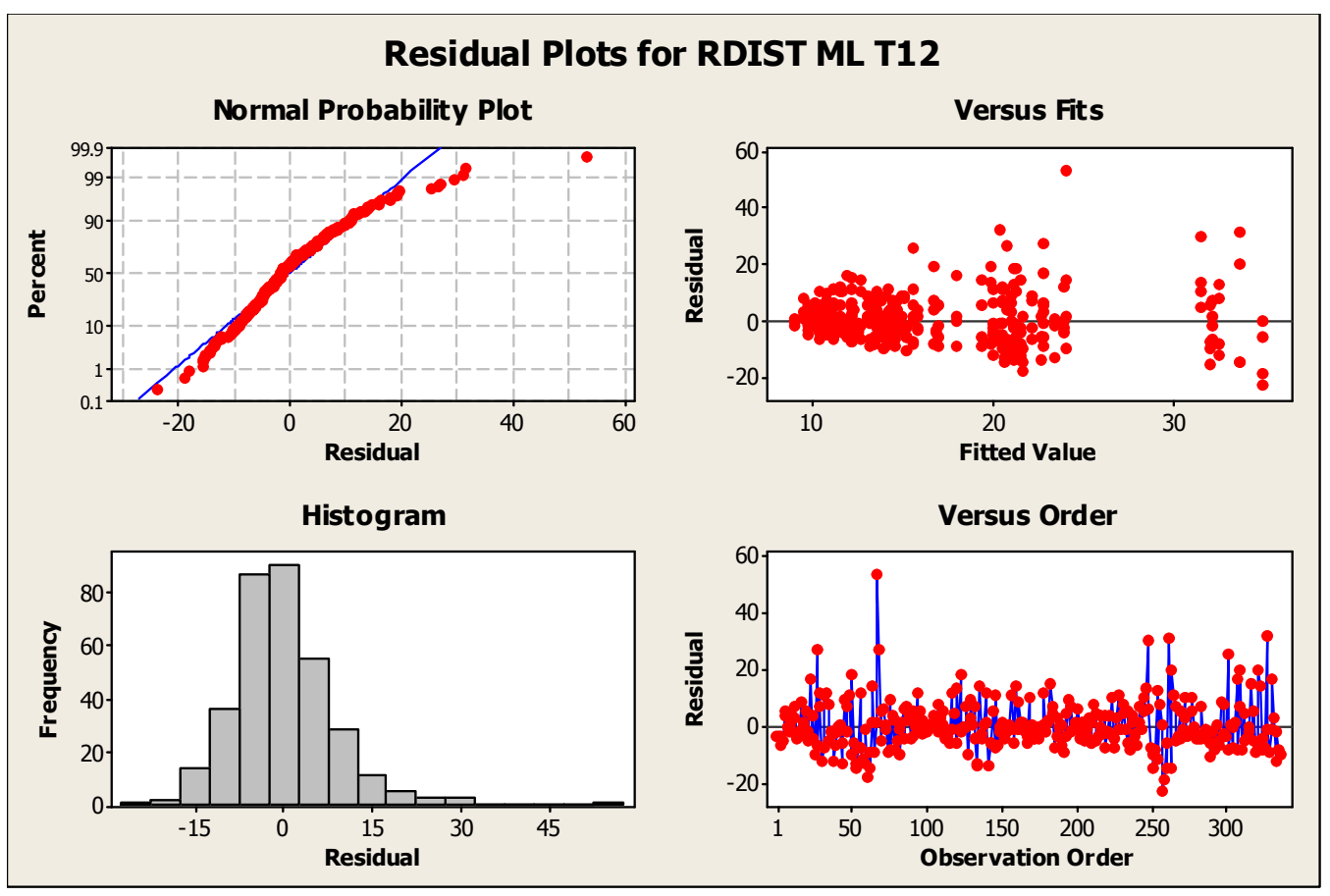

Figure 91: Residual plots for RDIST ML T12

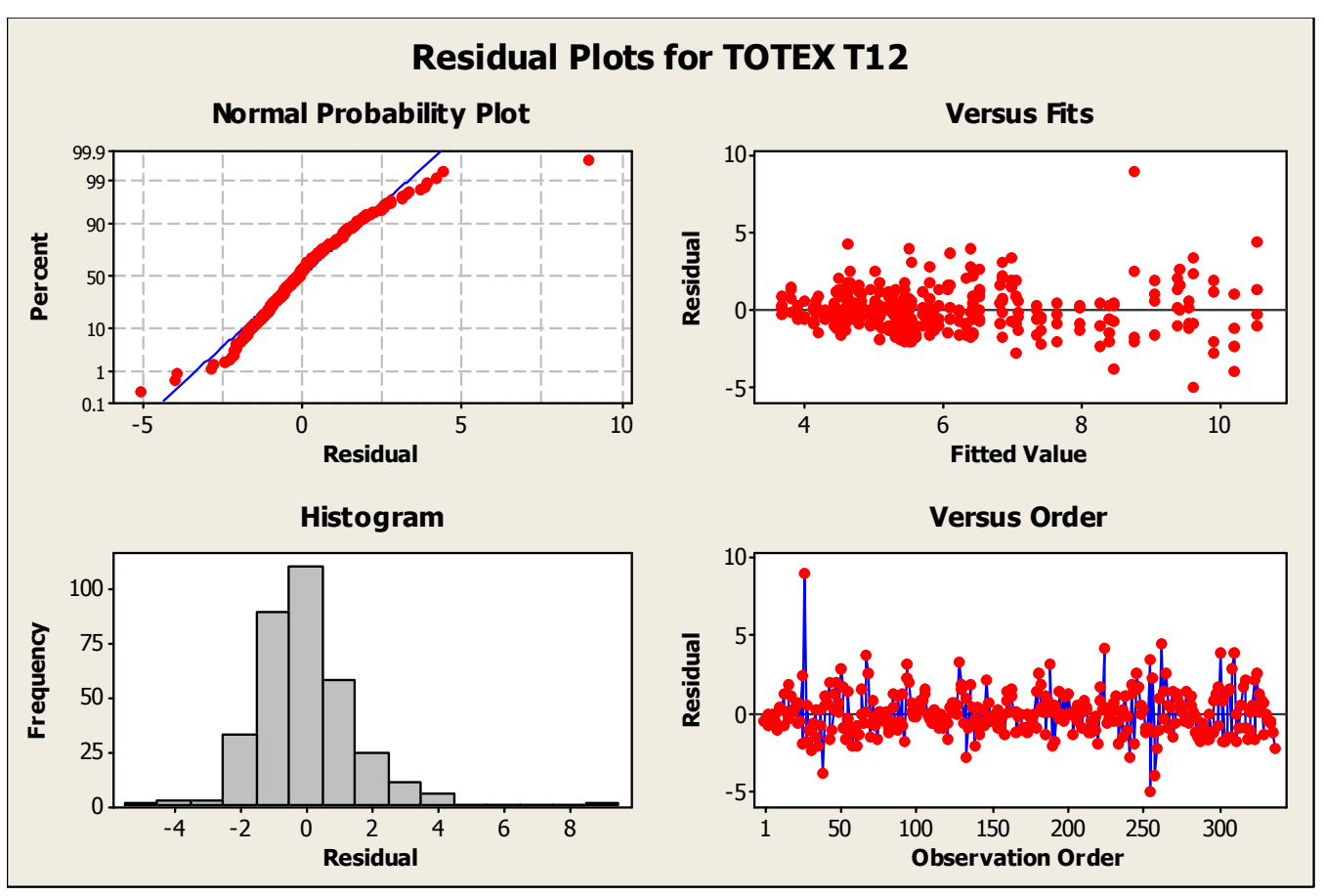

Figure 92: Residual plots for TOTEX T12 


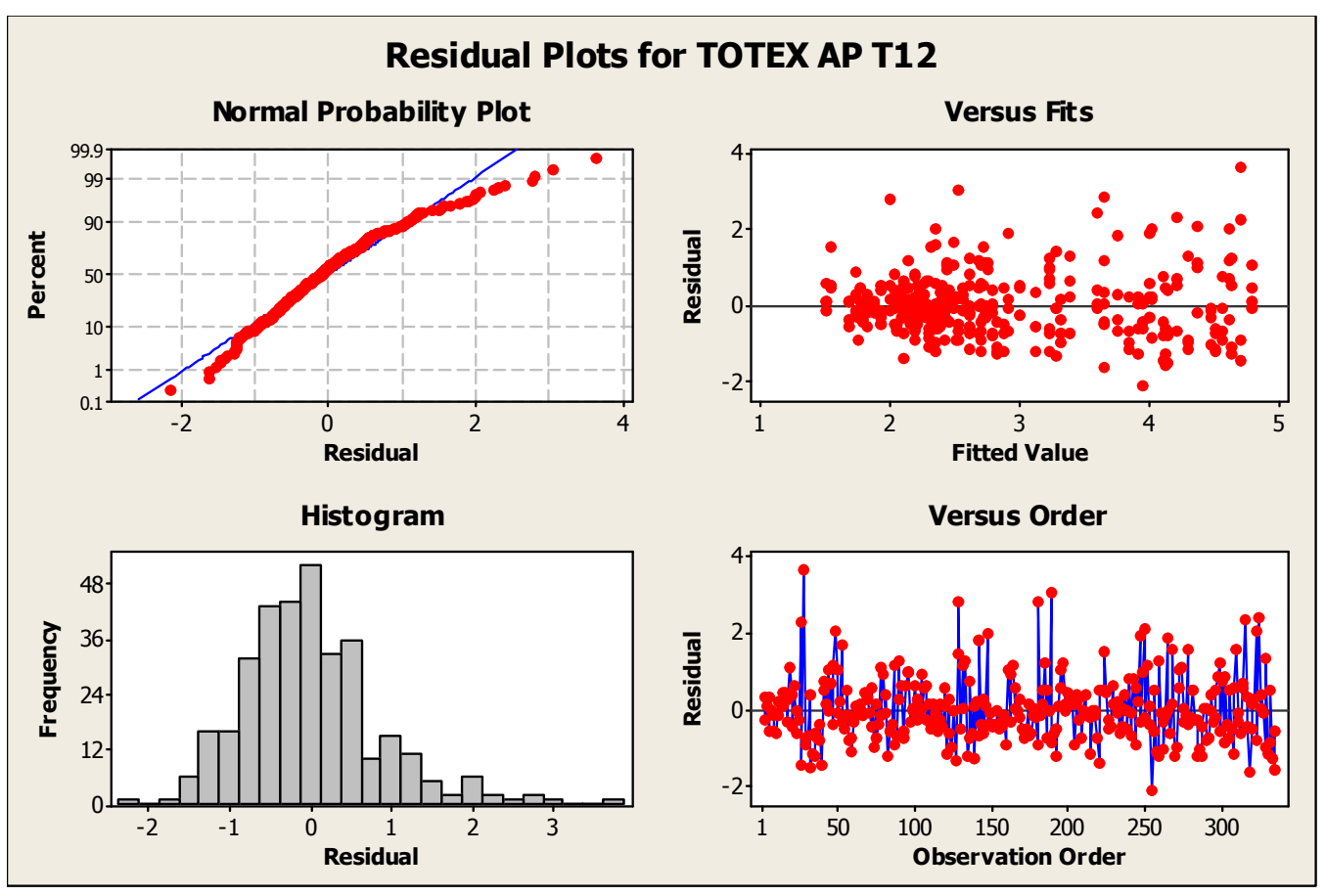

Figure 93: Residual plots for TOTEX AP T12

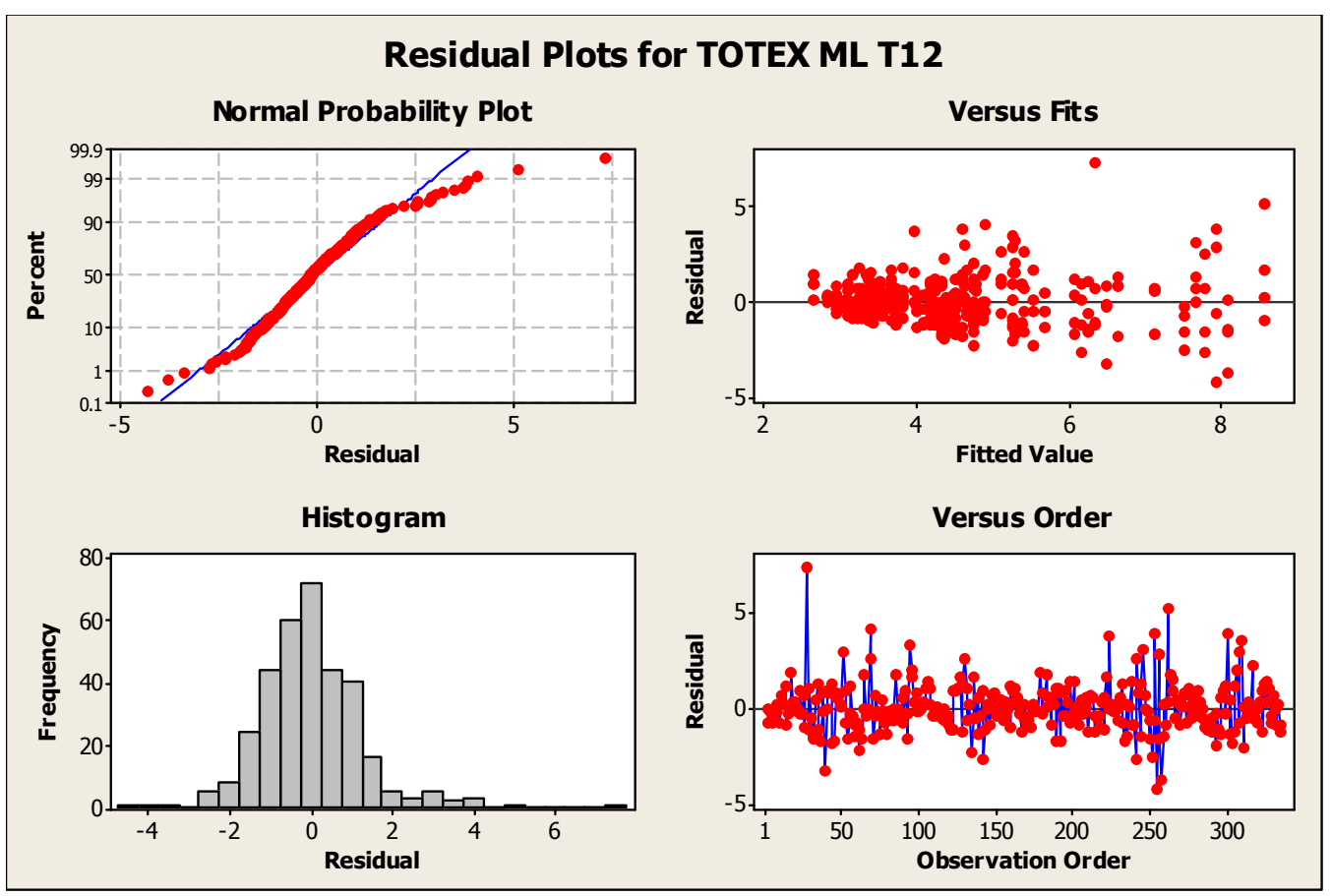

Figure 94: Residual plots for TOTEX ML T12 


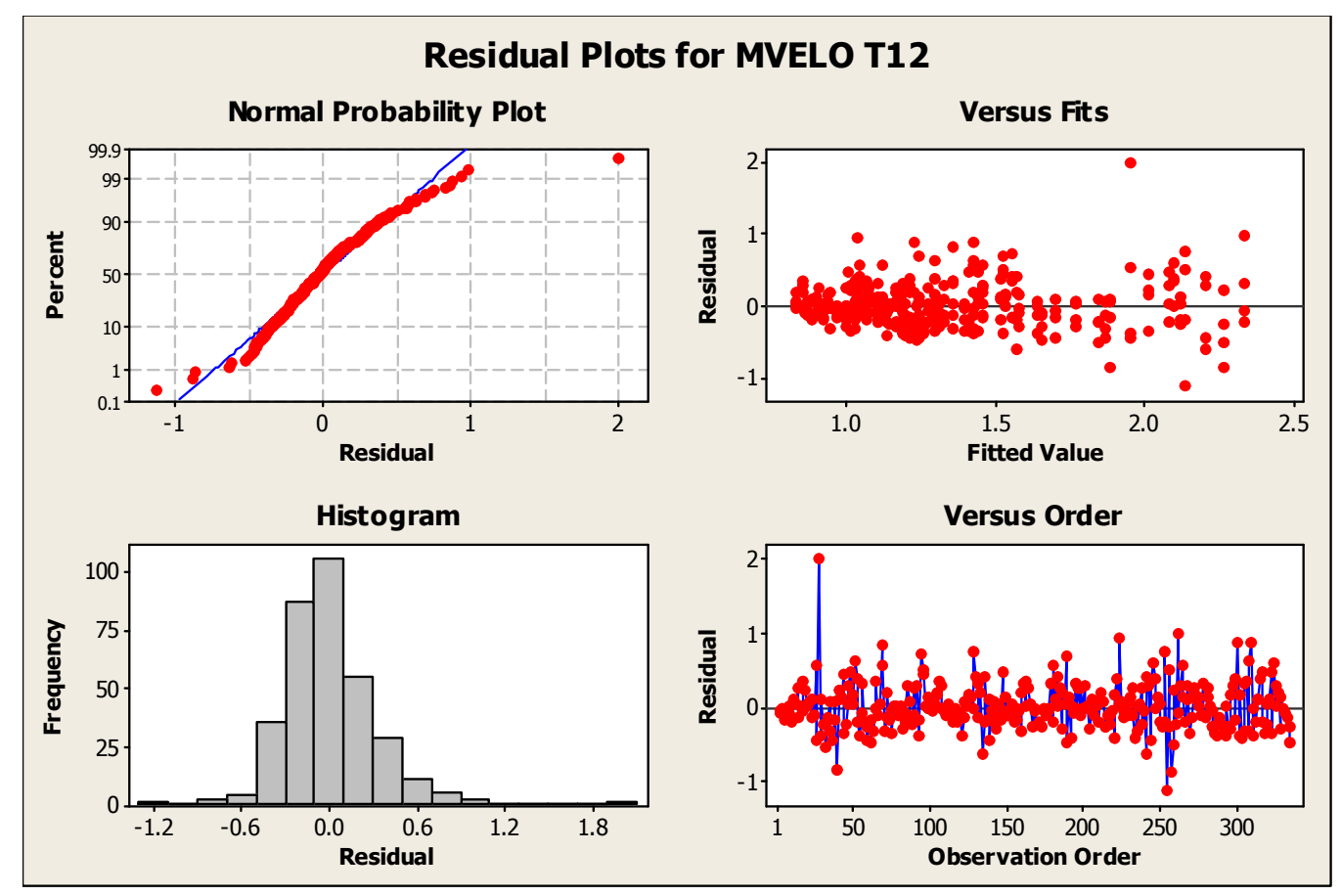

Figure 95: Residual plots for MVELO T12

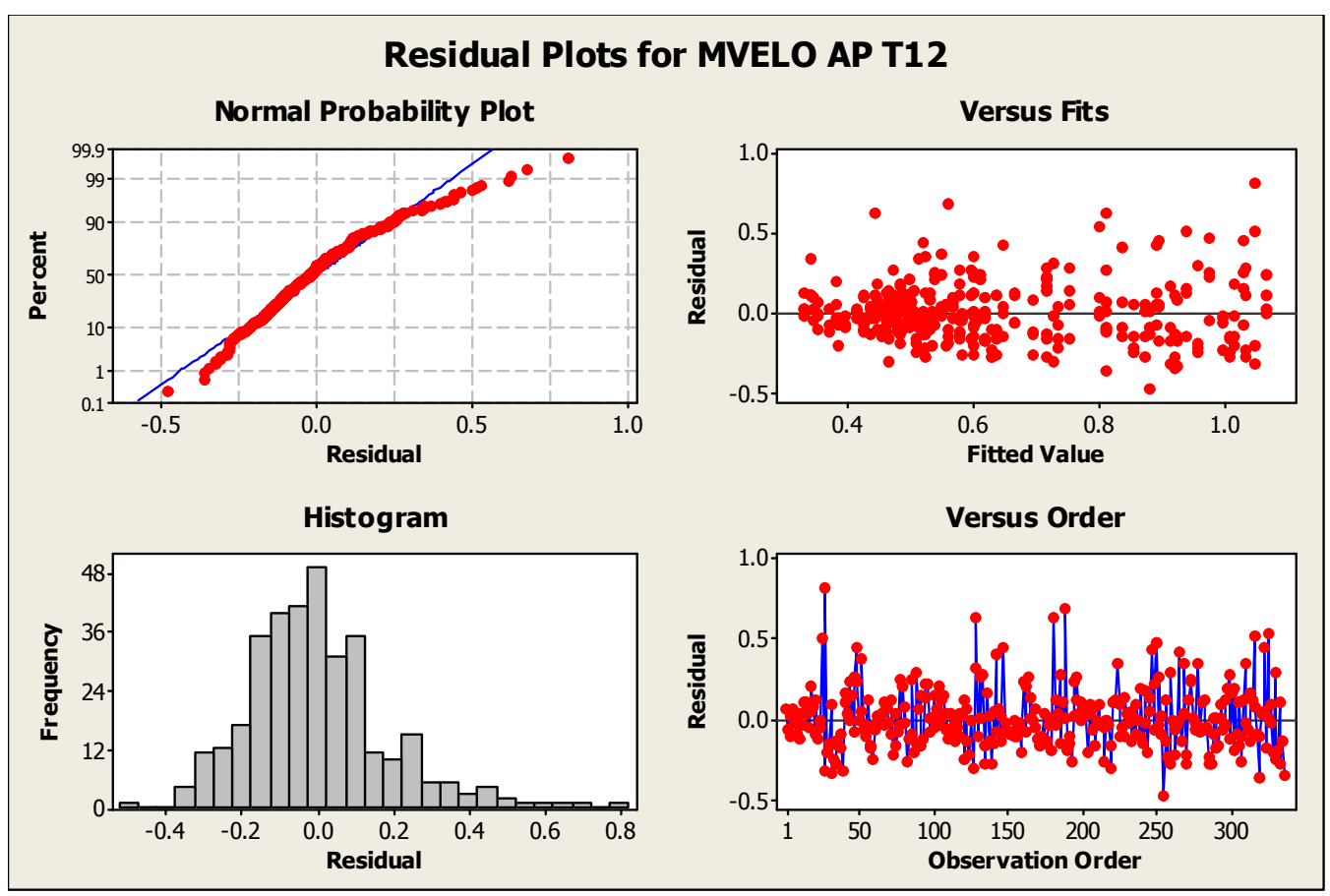

Figure 96: Residual plots for MVELO AP T12 


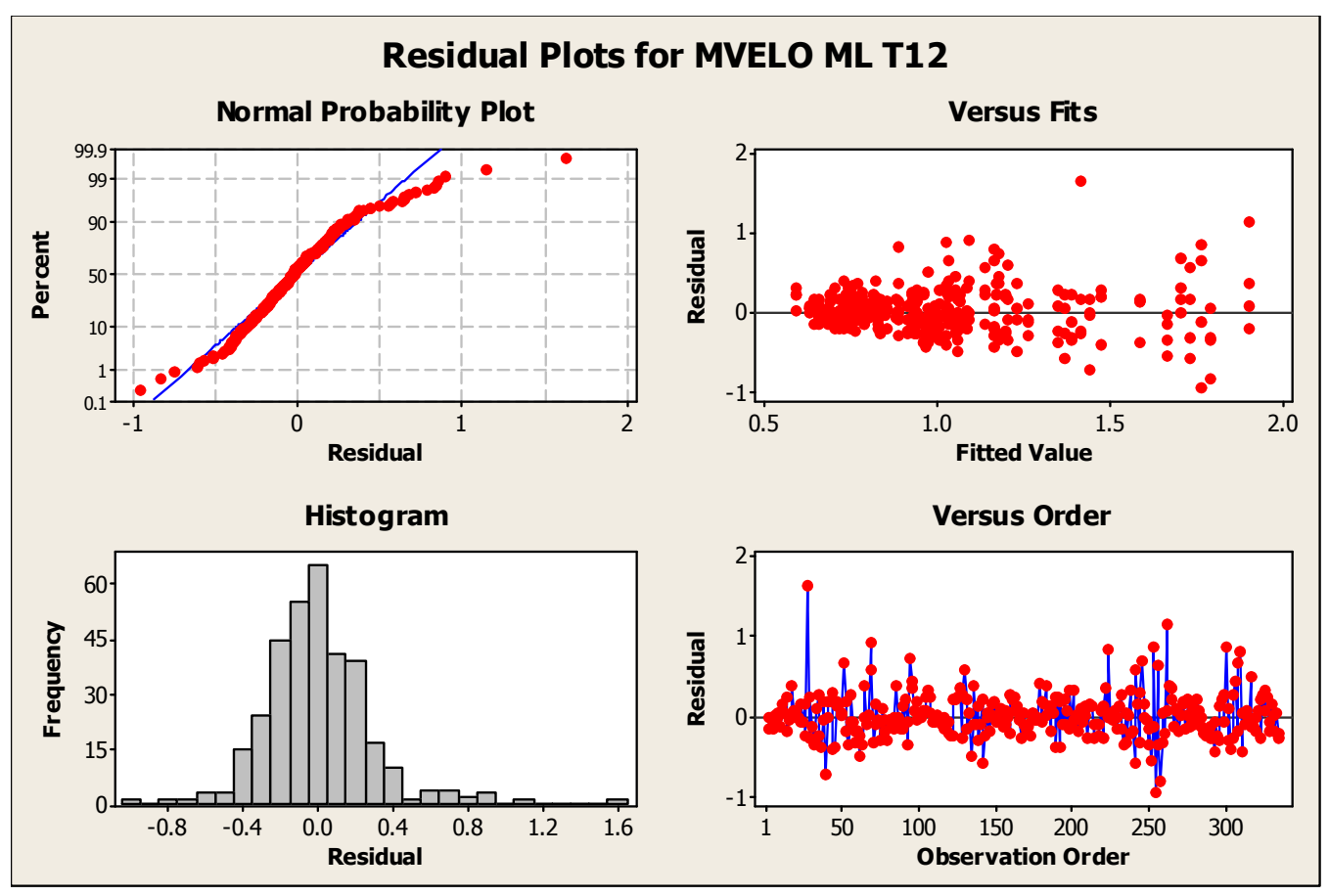

Figure 97: Residual plots for MVELO ML T12

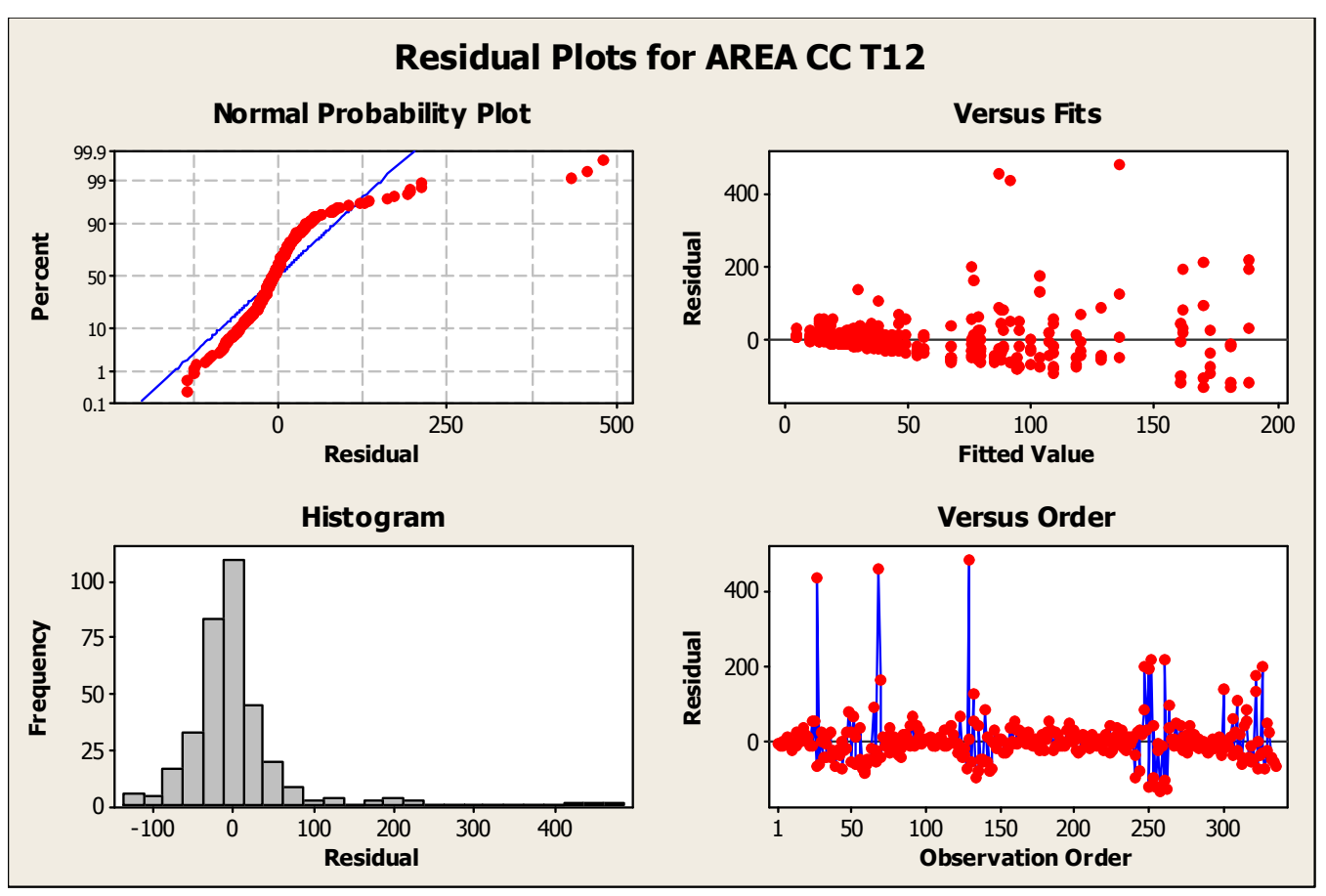

Figure 98: Residual plots for AREA CC T12 


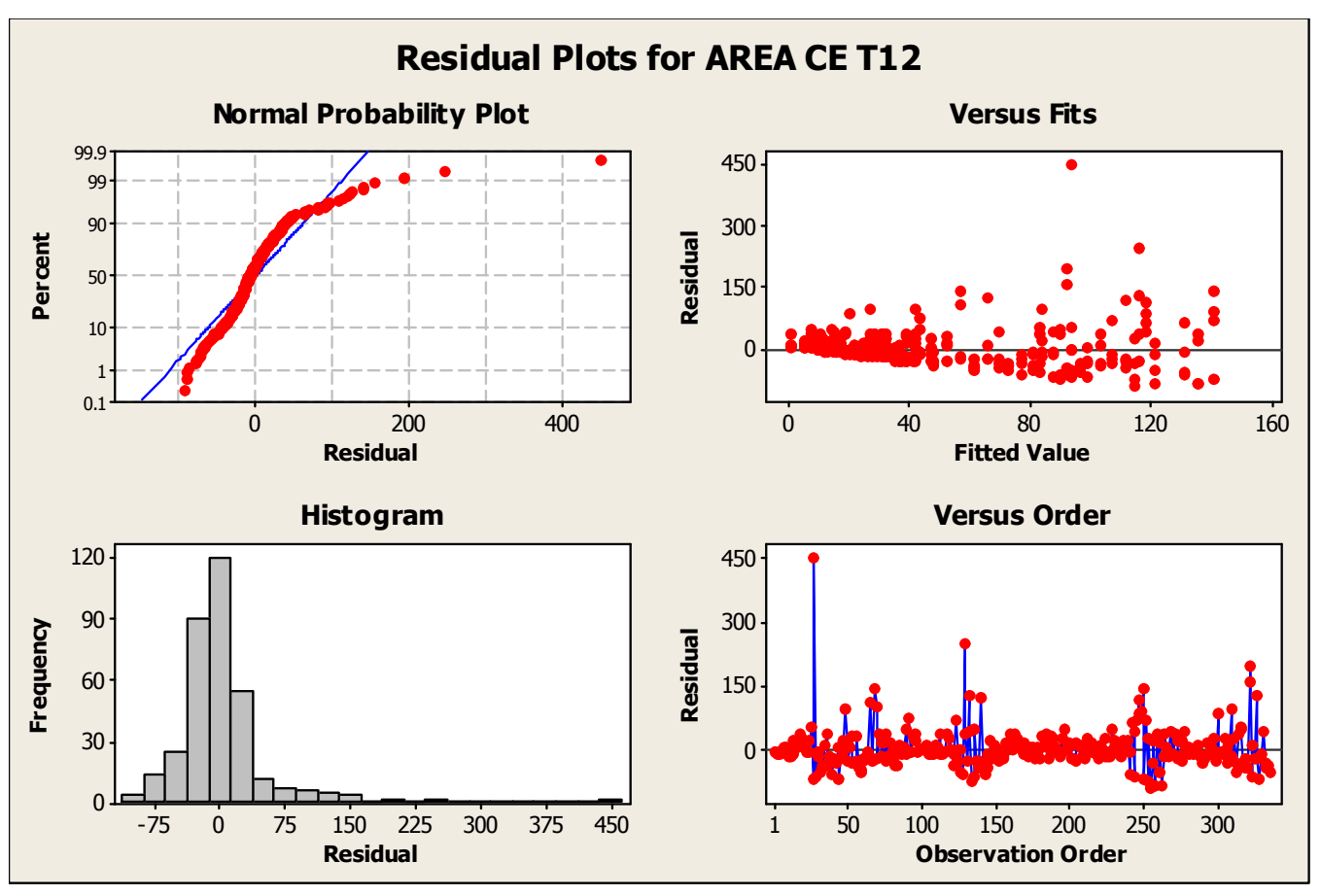

Figure 99: Residual plots for AREA CE T12

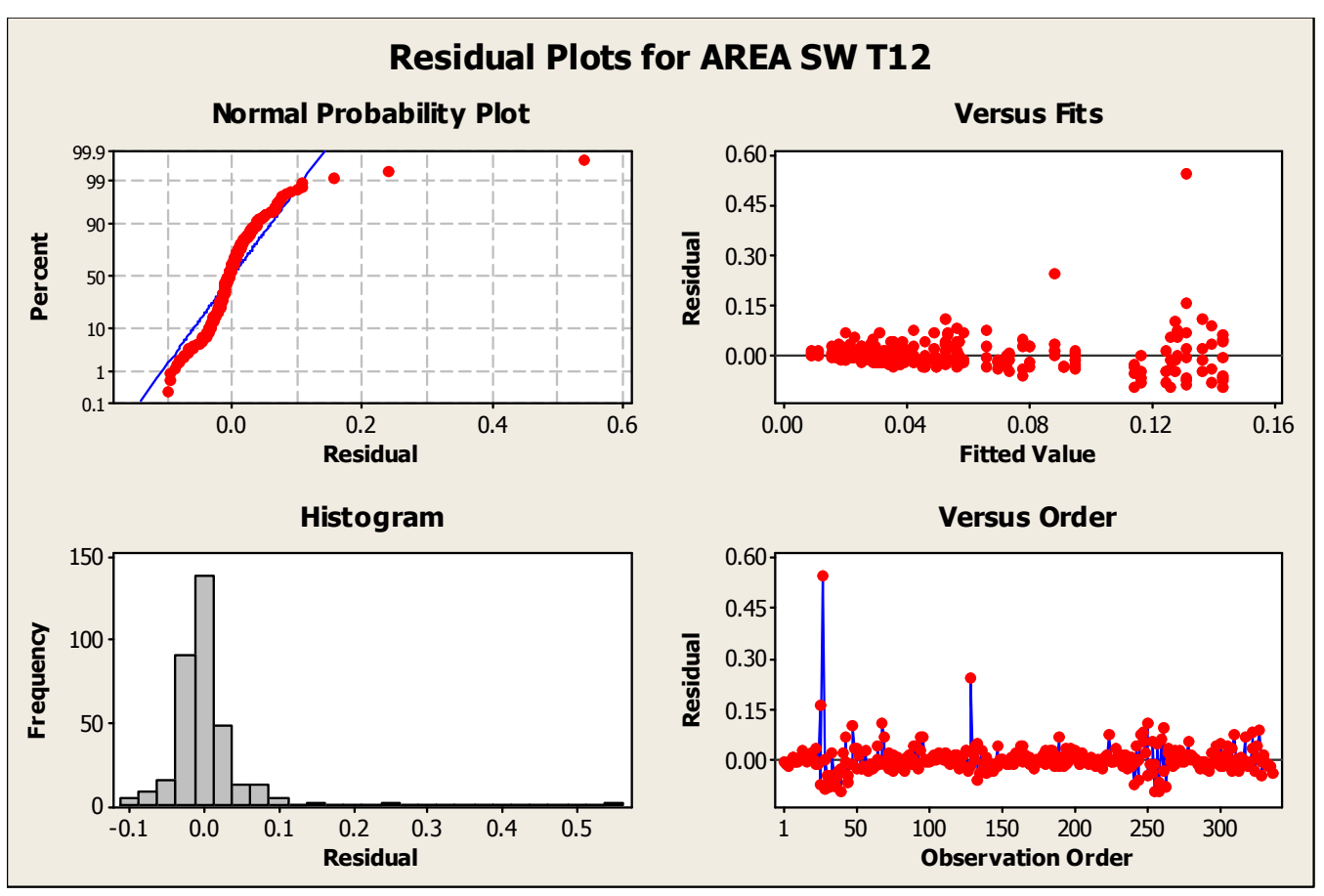

Figure 100: Residual plots for AREA SW T12 


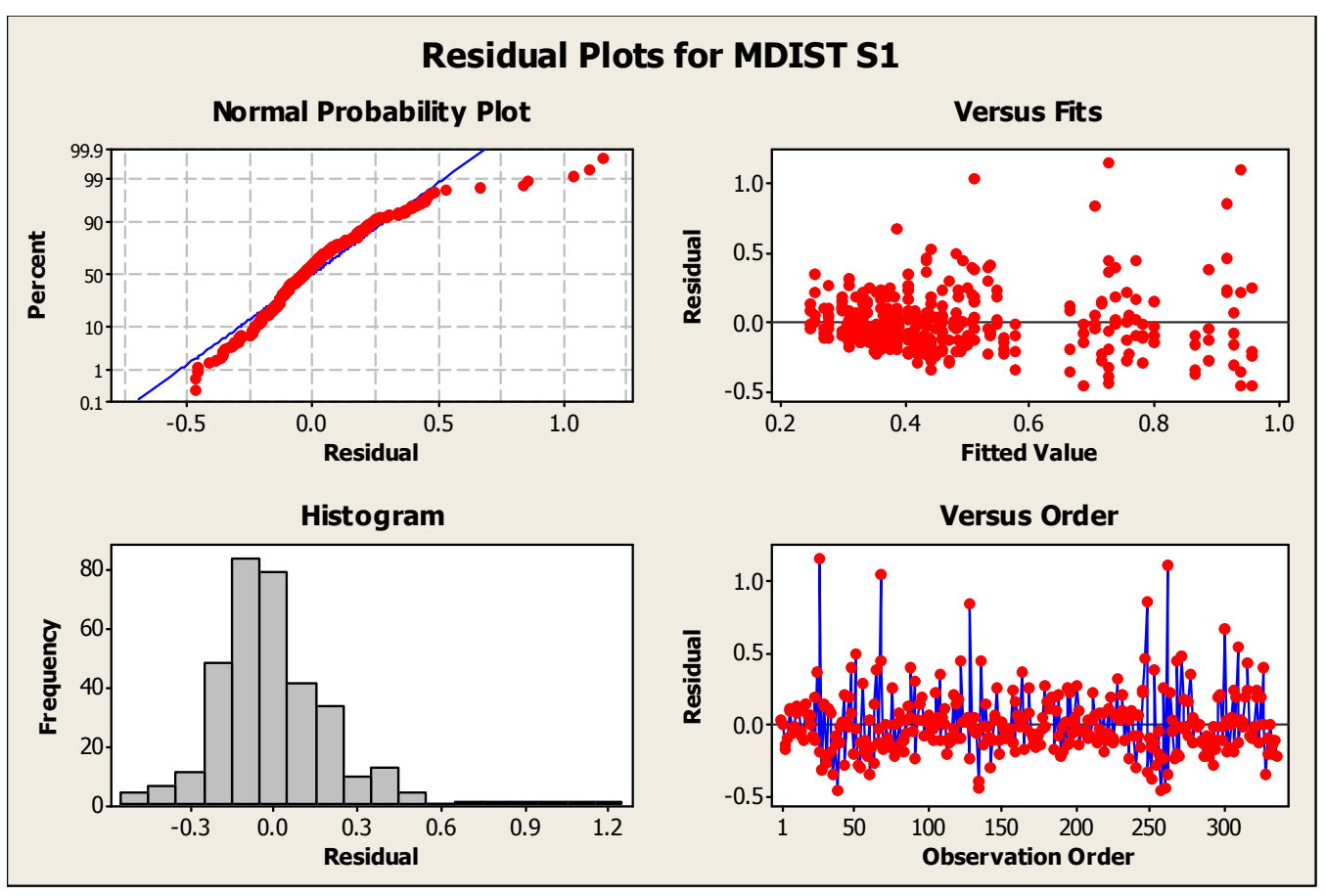

Figure 101: Residual plots for MDIST S1

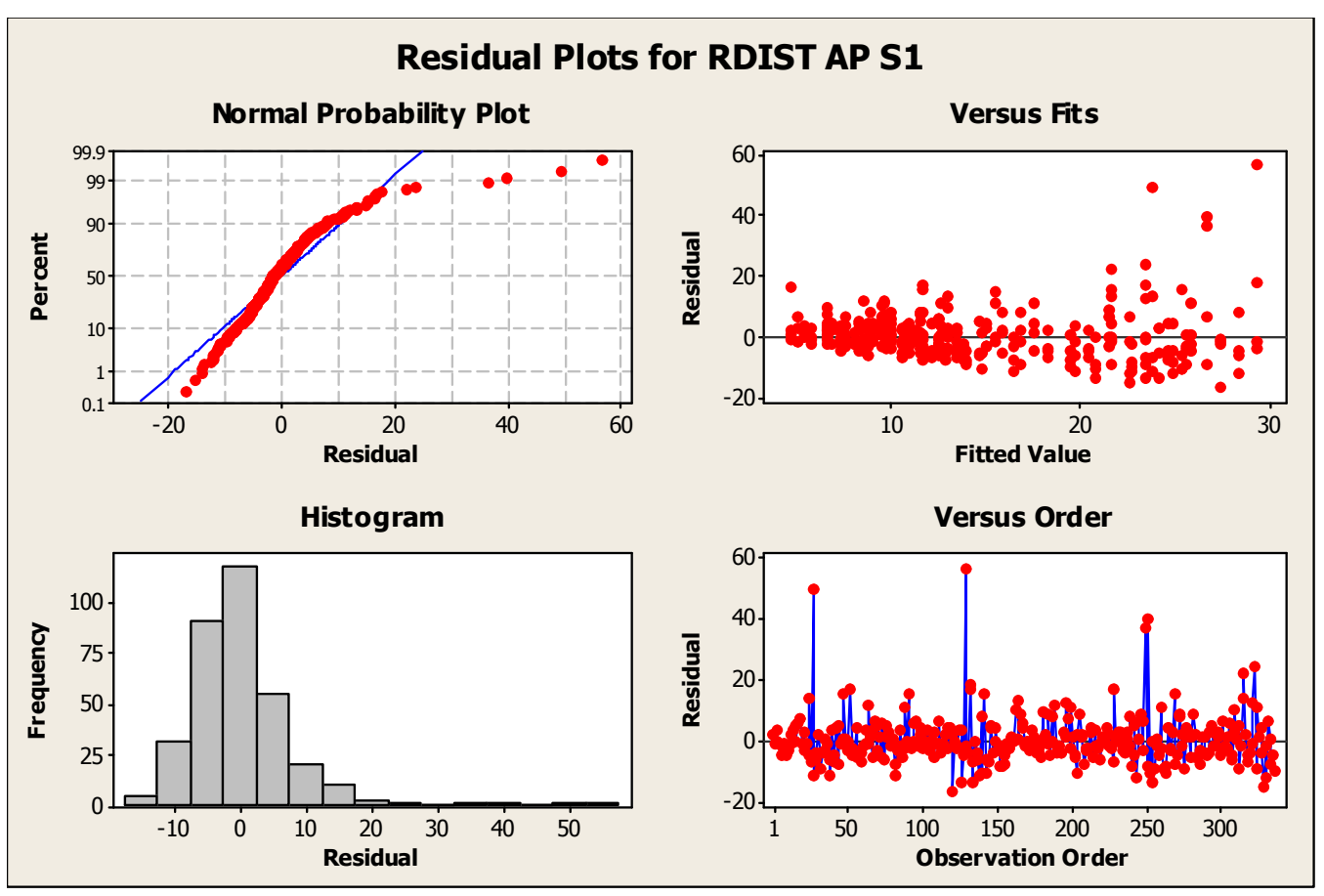

Figure 102: Residual plots for MDIST AP S1 


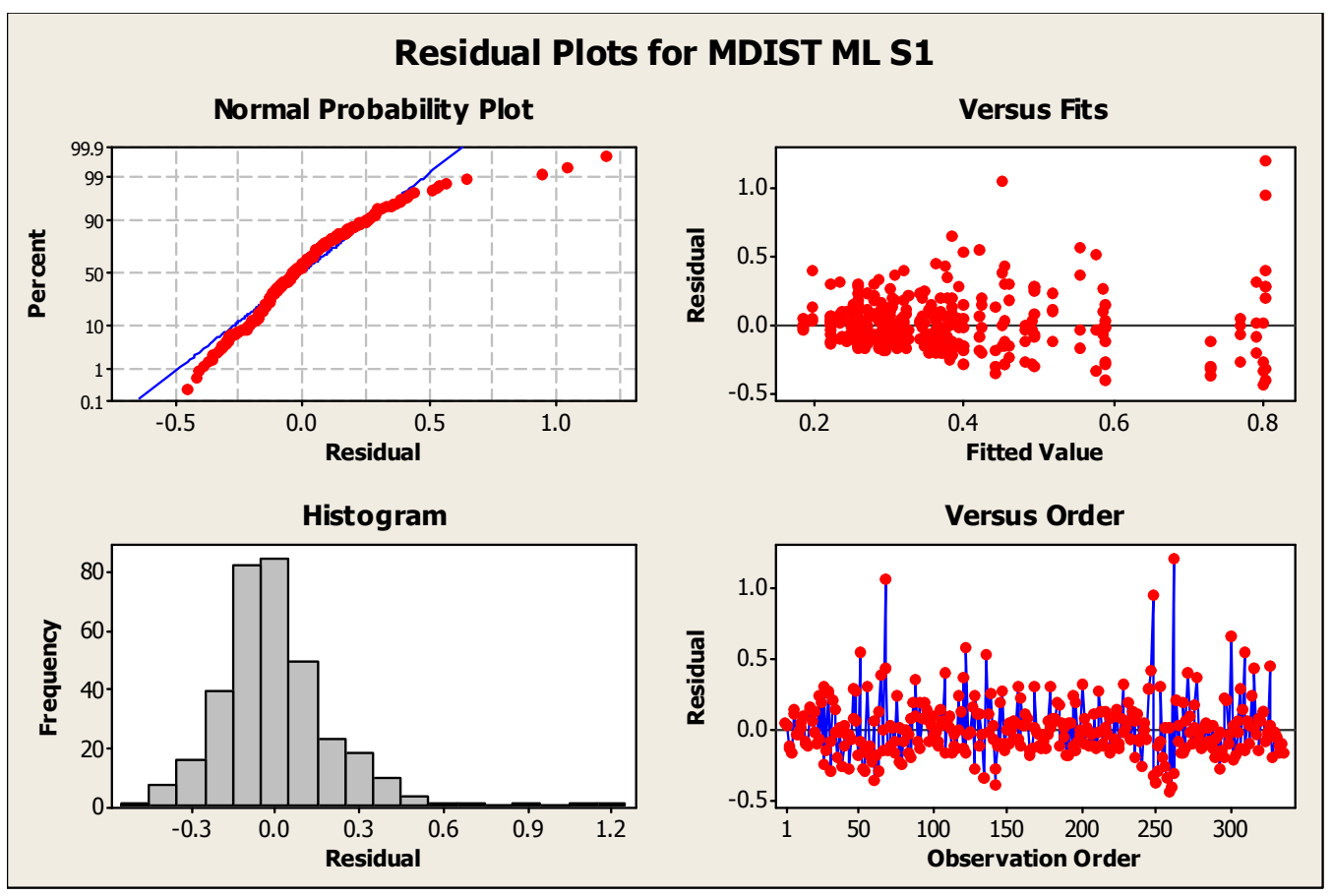

Figure 103: Residual plots for MDIST ML S1

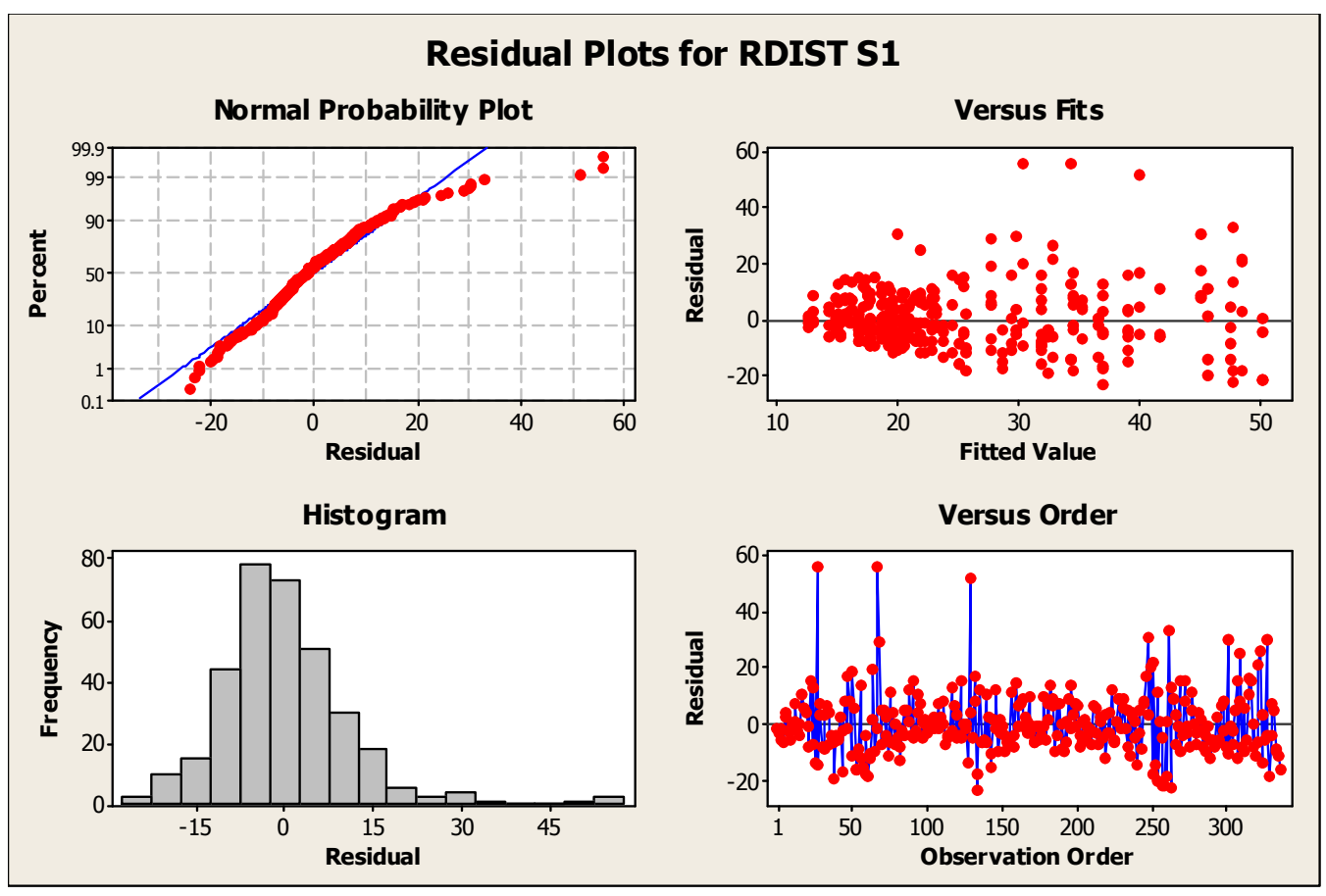

Figure 104: Residual plots for RDIST S1 


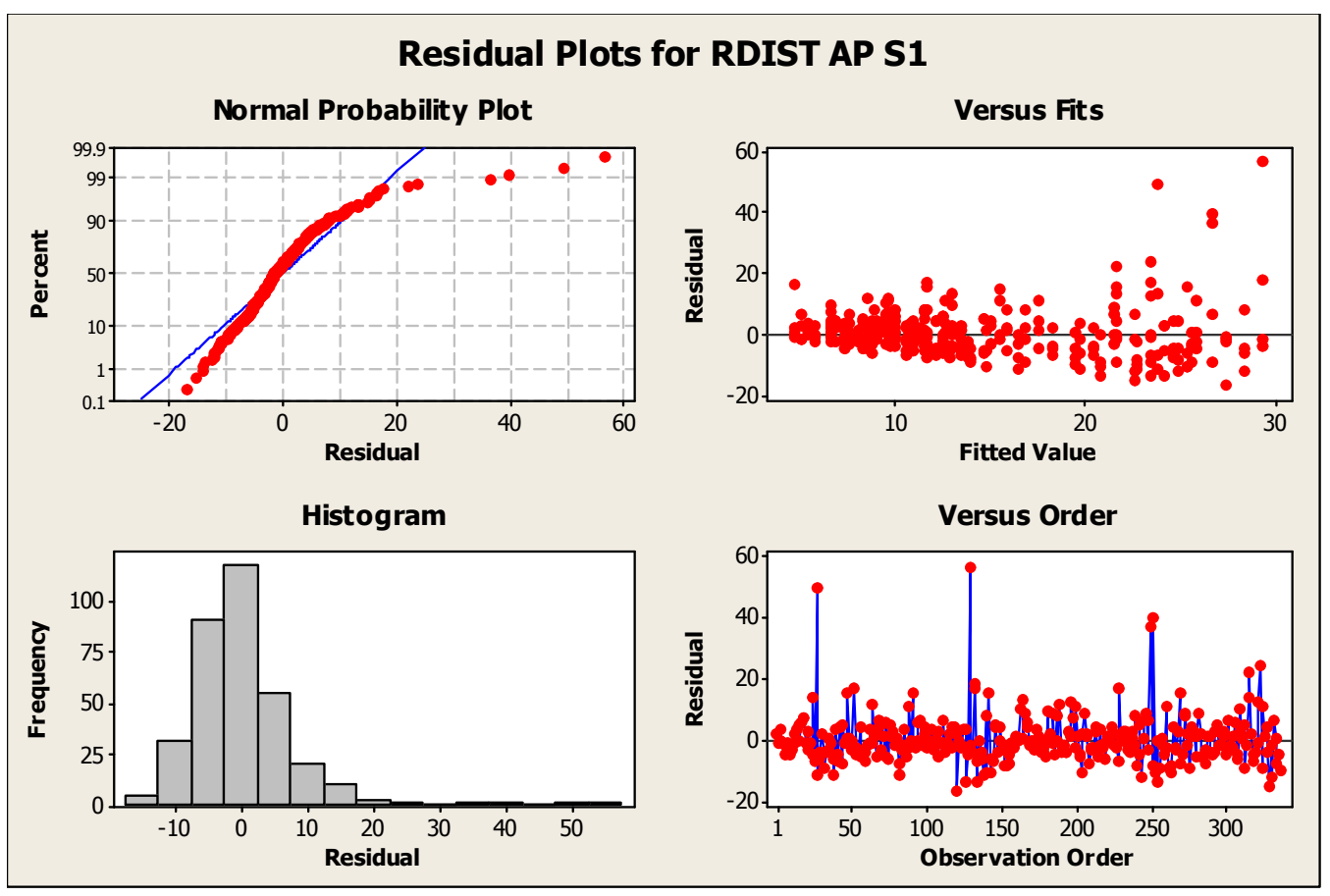

Figure 105: Residual plots for RDIST AP S1

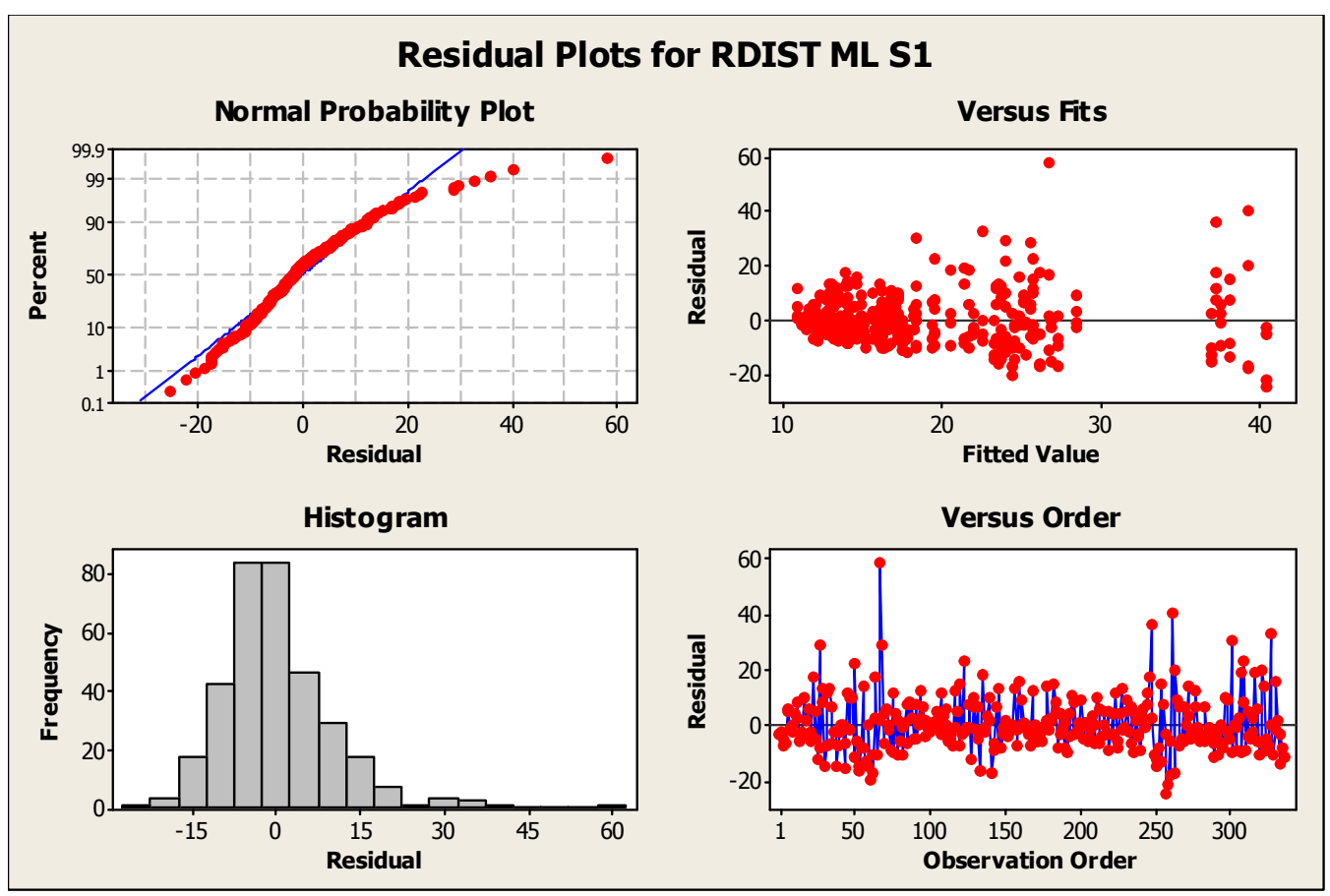

Figure 106: Residual plots for RDIST ML S1 


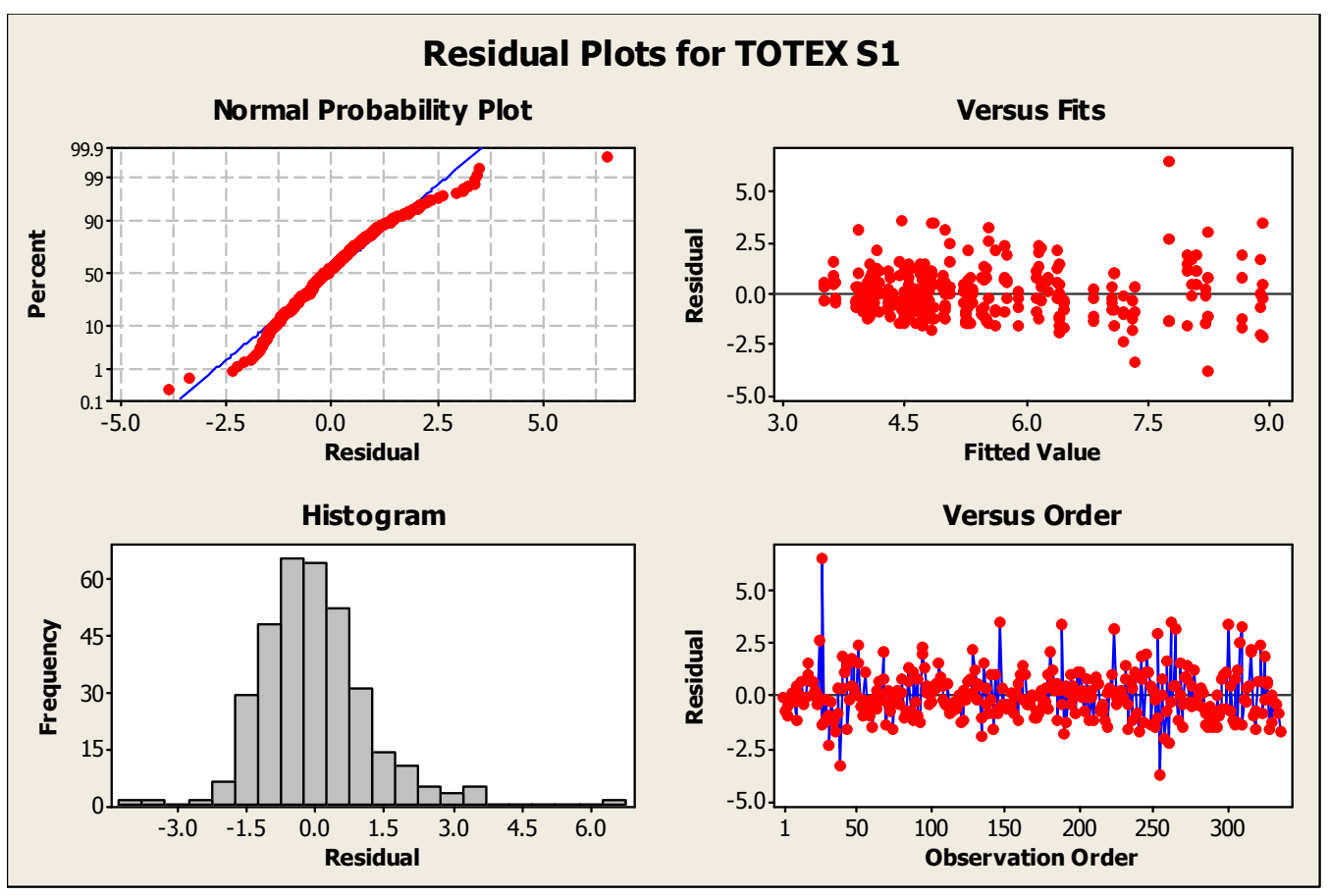

Figure 107: Residual plots for TOTEX S1

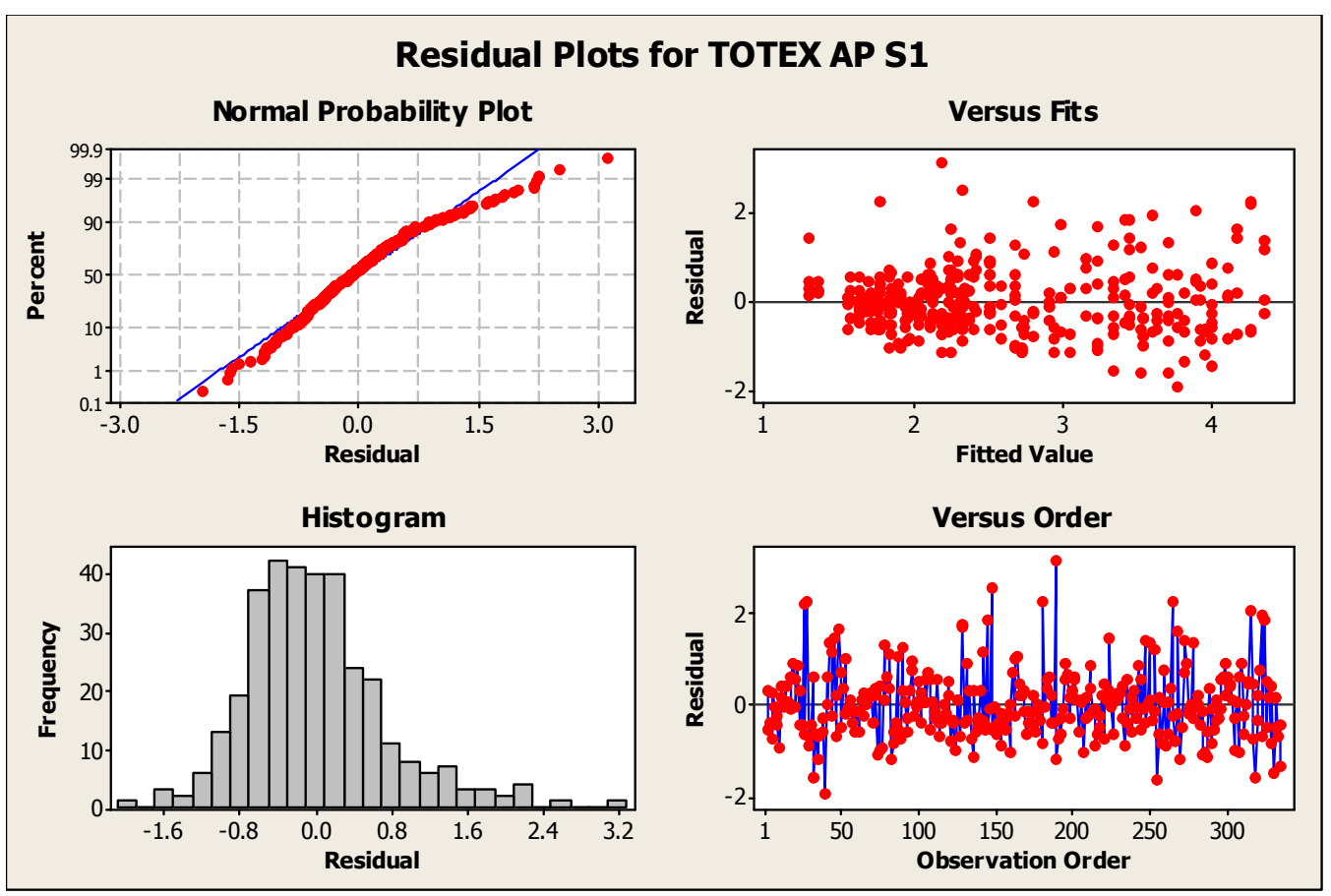

Figure 108: Residual plots for TOTEX AP S1 


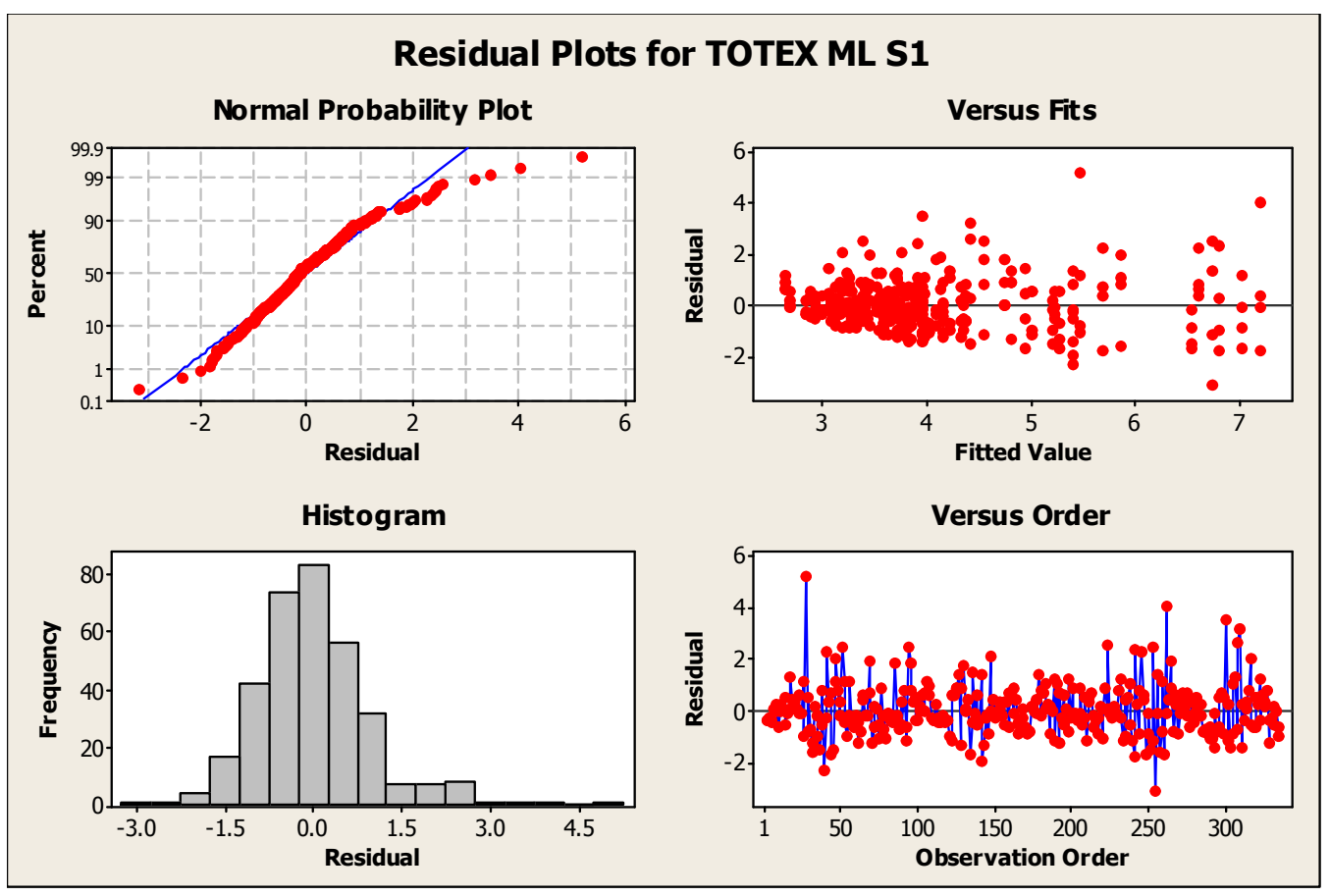

Figure 109: Residual plots for TOTEX ML S1

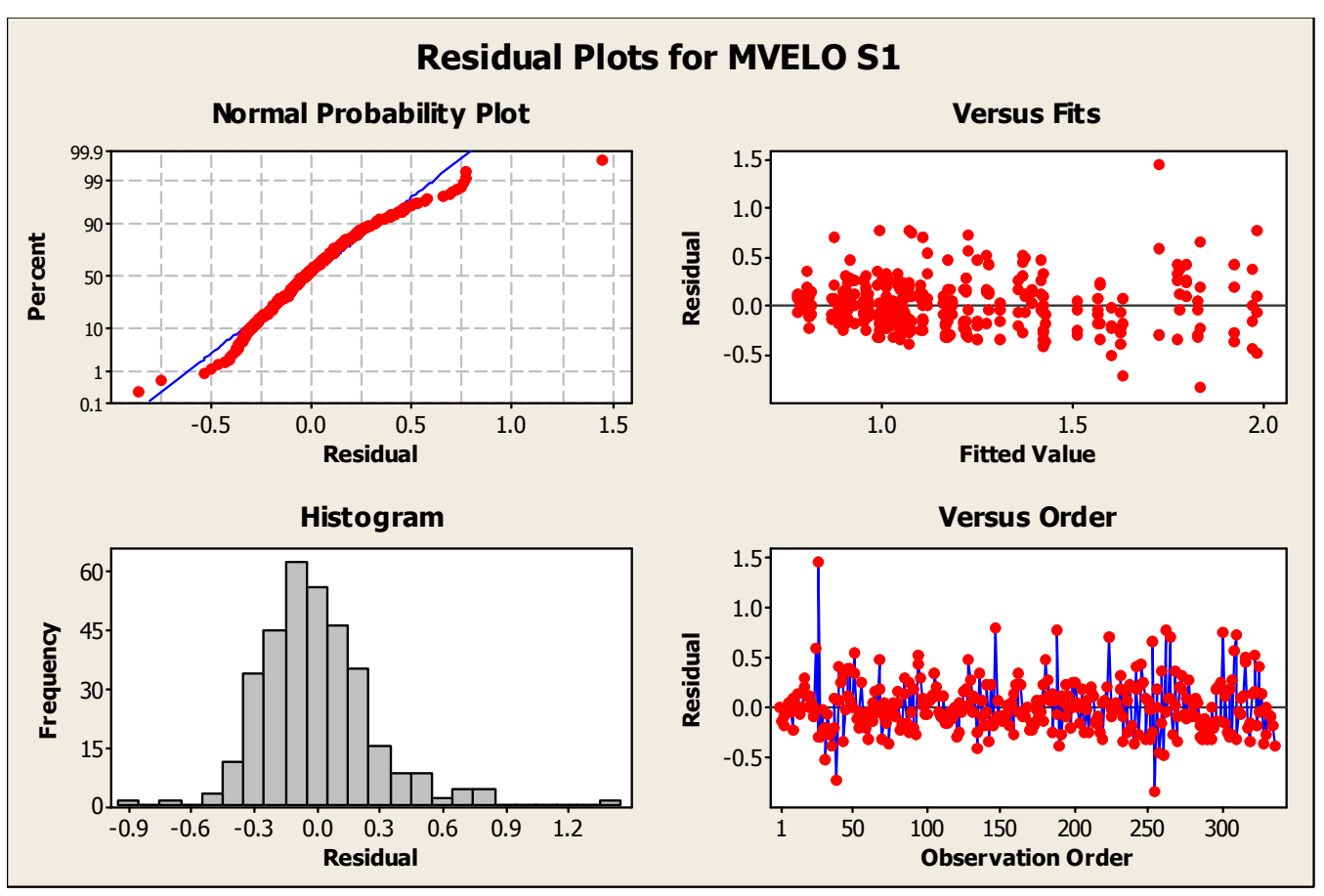

Figure 110: Residual plots for MVELO S1 


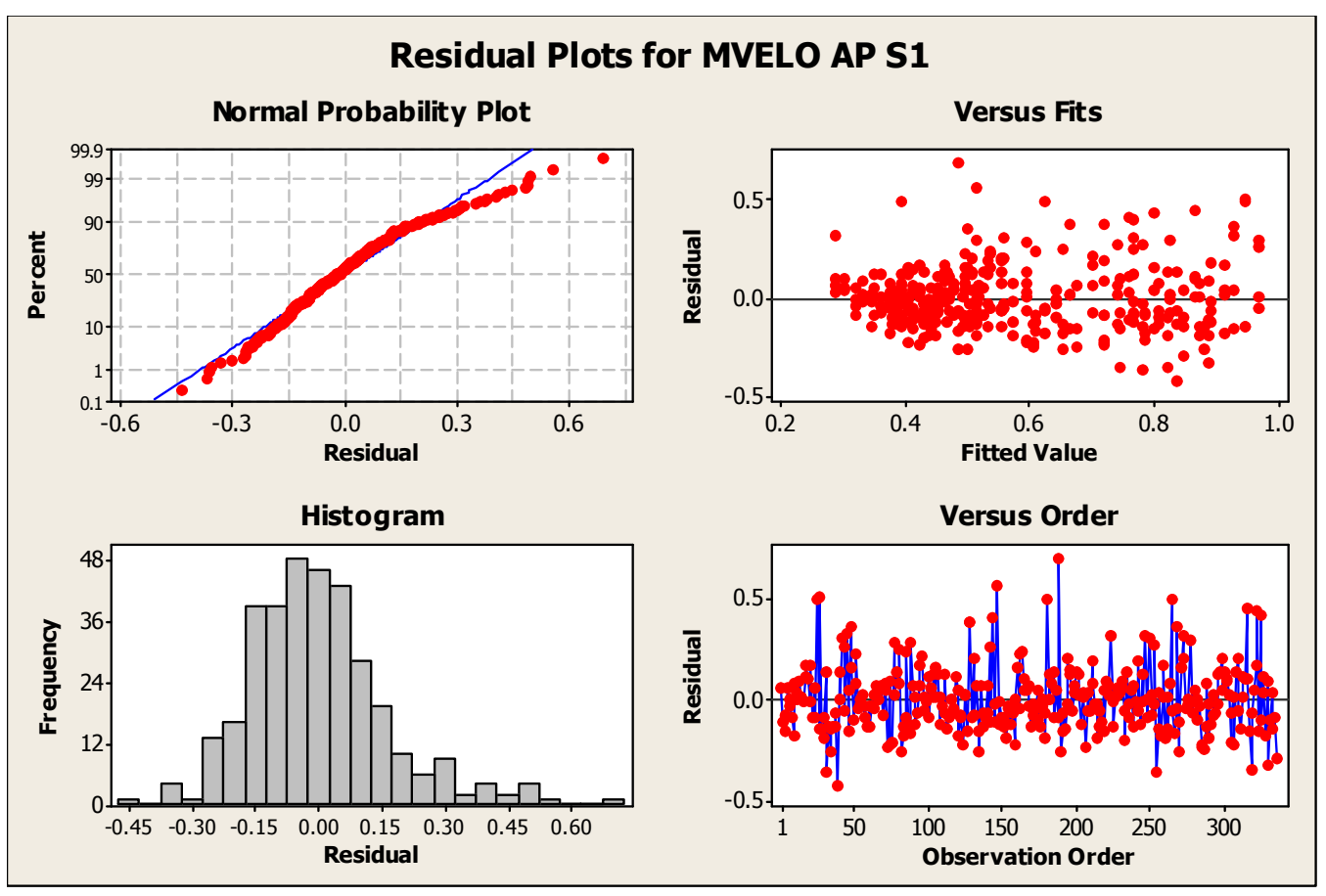

Figure 111: Residual plots for MVELO AP S1

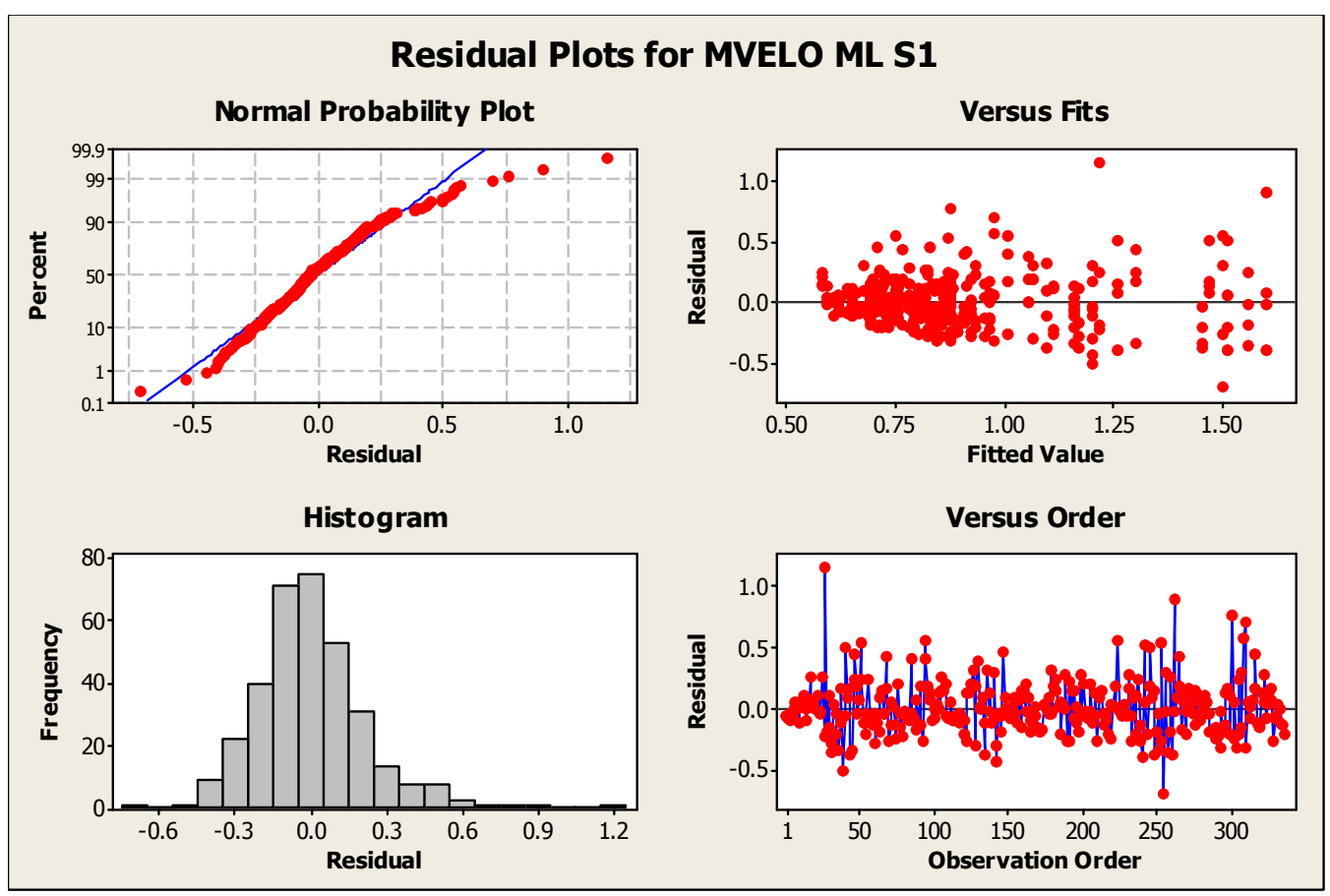

Figure 112: Residual plots for MVELO ML S1 


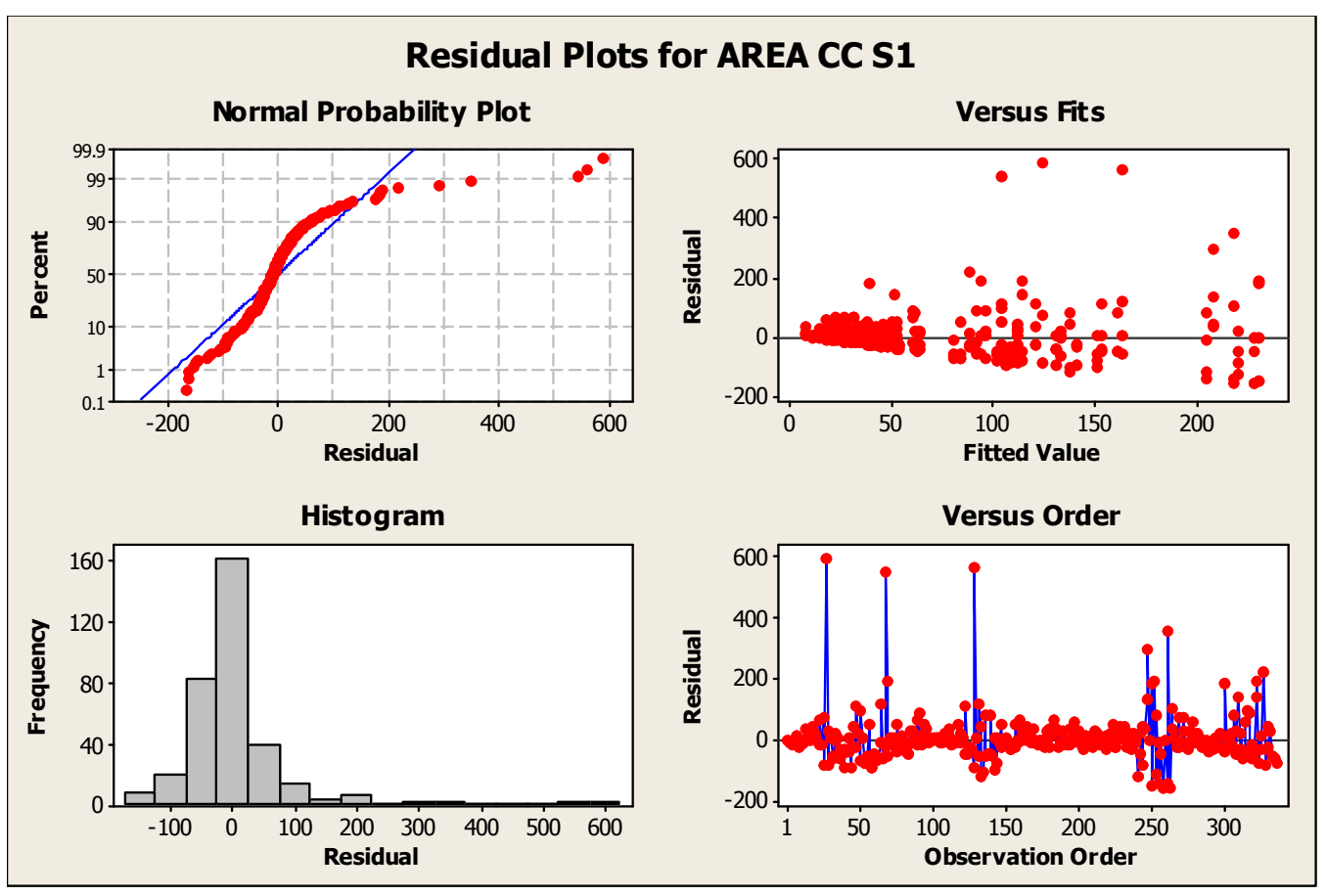

Figure 113: Residual plots for AREA CC S1

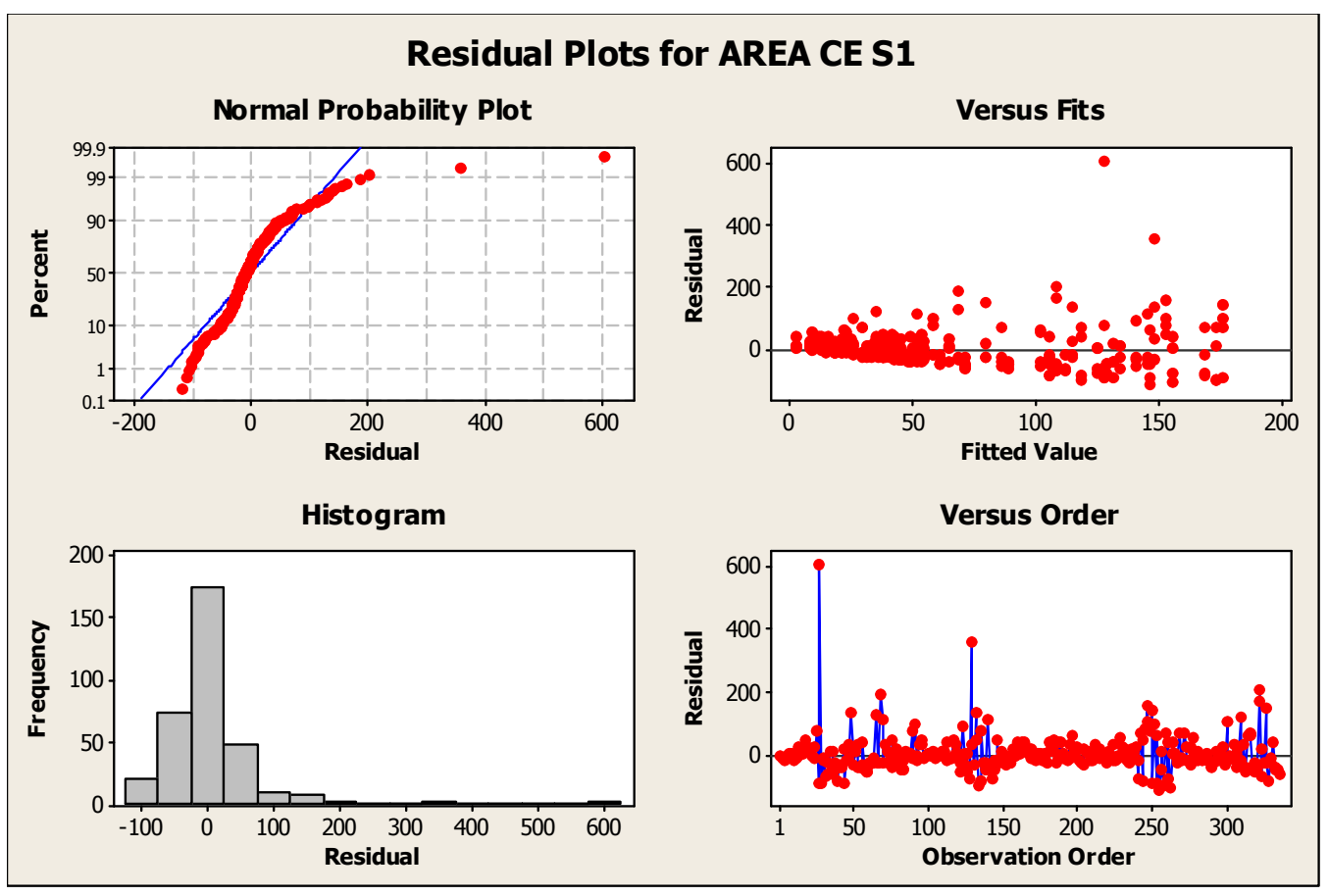

Figure 114: Residual plots for AREA CE S1 


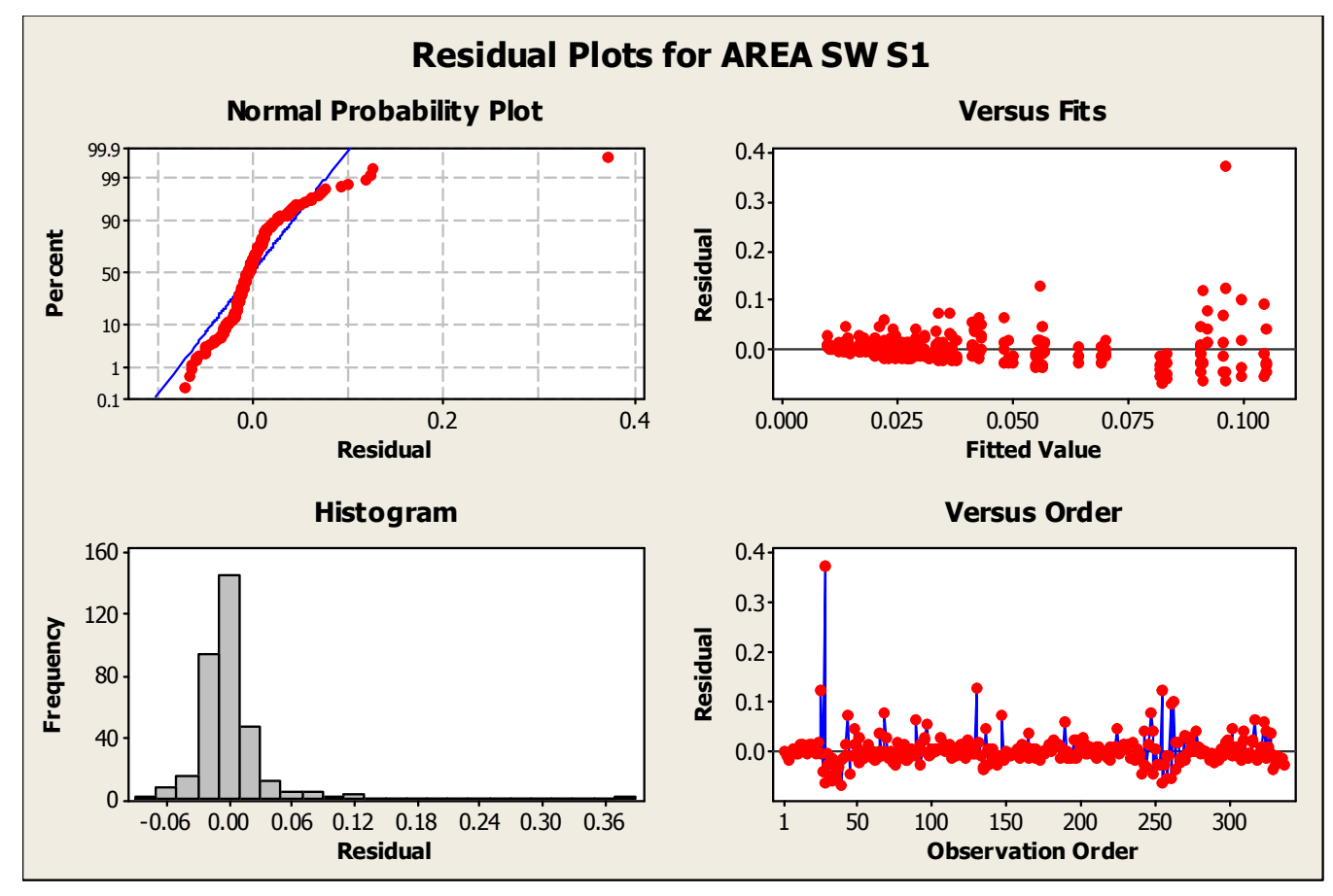

Figure 115: Residual plots for AREA SW S1 


\section{APPENDIX C. EFFECTS OF GROUND ON SEGMENTAL STABILITY (SUPPLEMENT)}

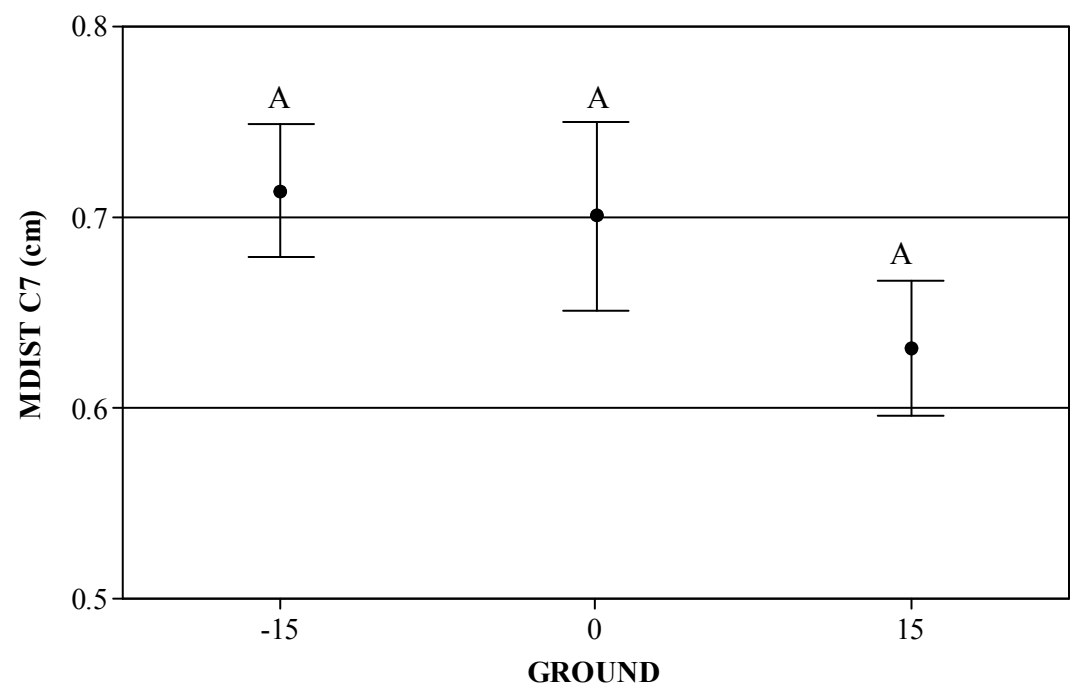

Figure 116: Effects of GROUND on C7 MDIST

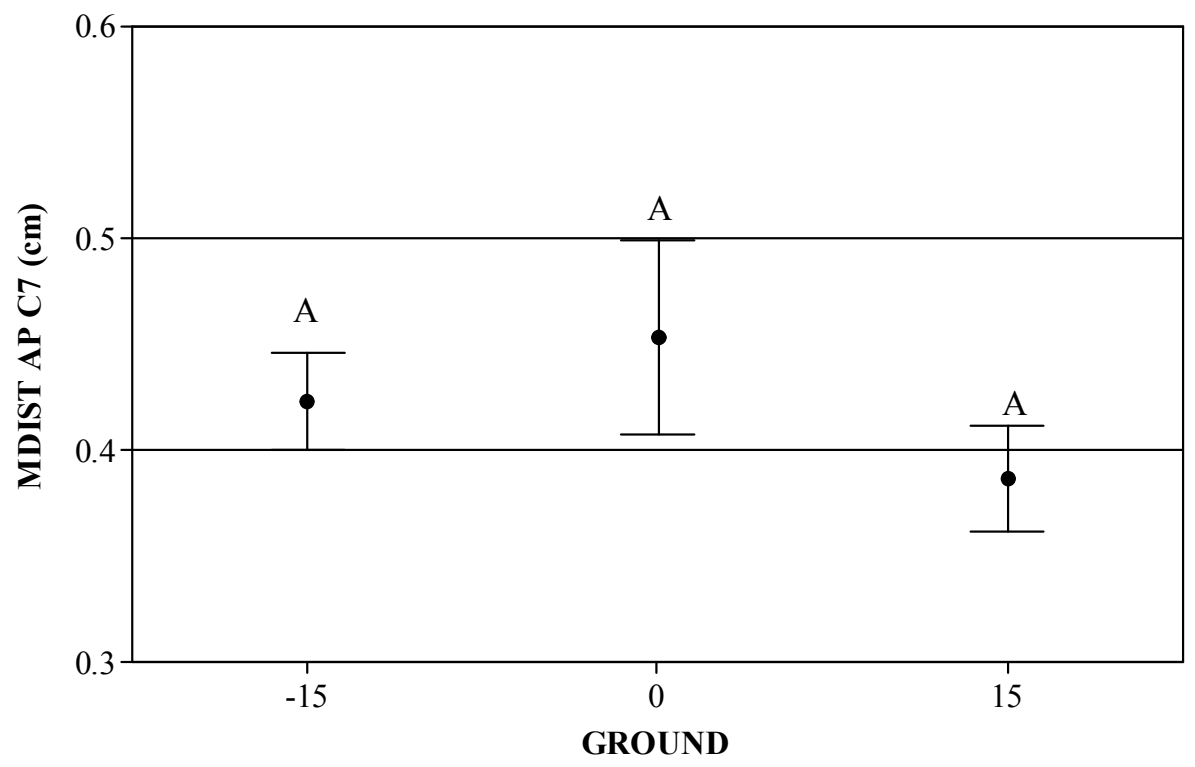

Figure 117: Effects of GROUND on C7 MDIST AP 


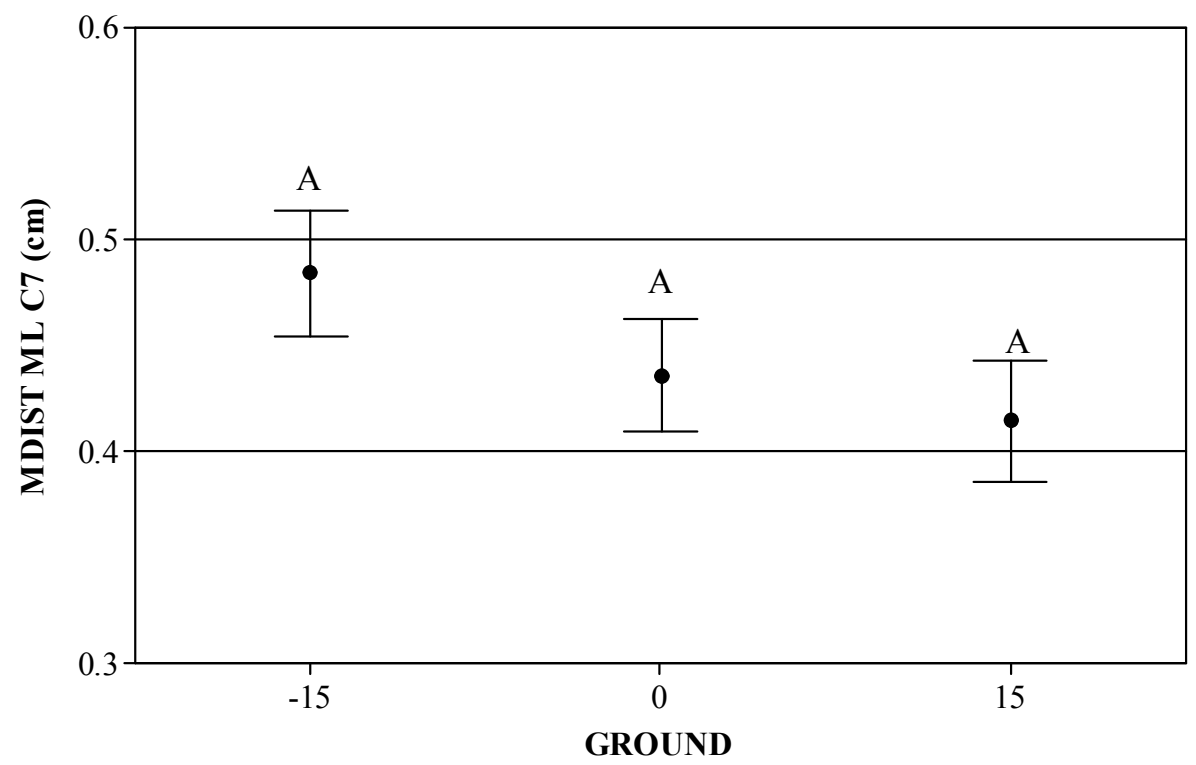

Figure 118: Effects of GROUND on C7 MDIST ML

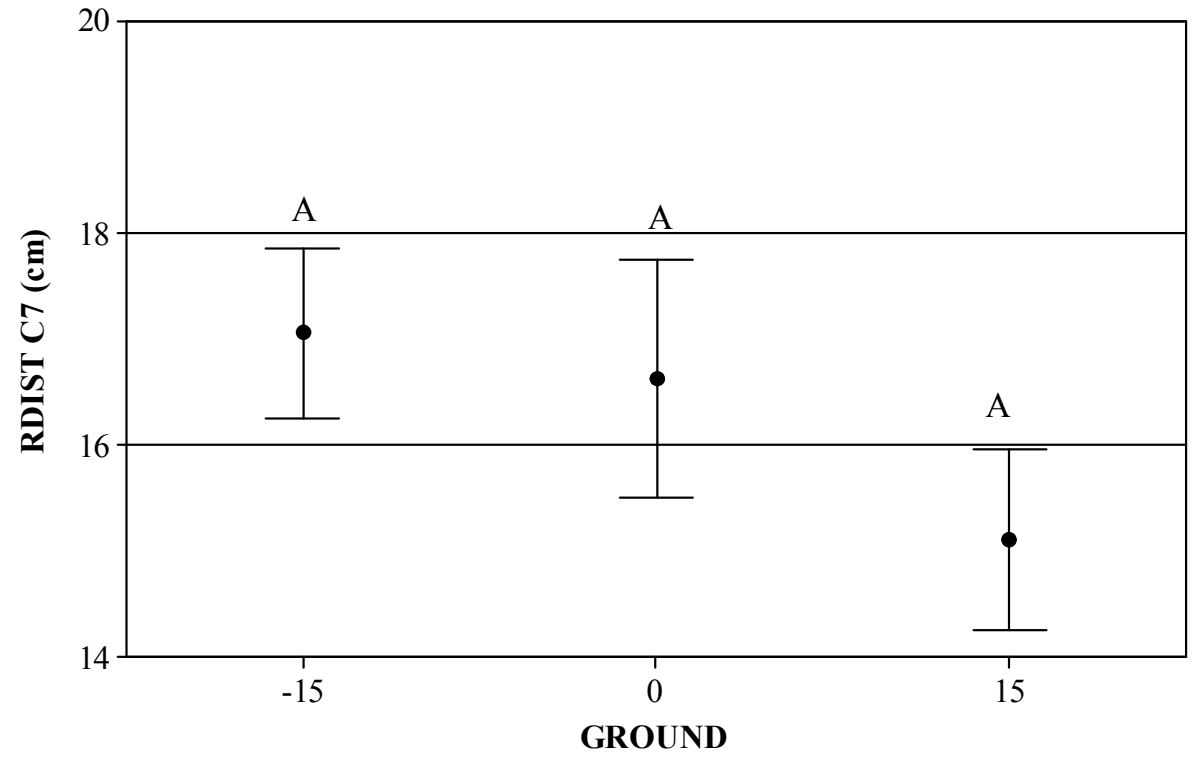

Figure 119: Effects of GROUND on C7 RDIST 


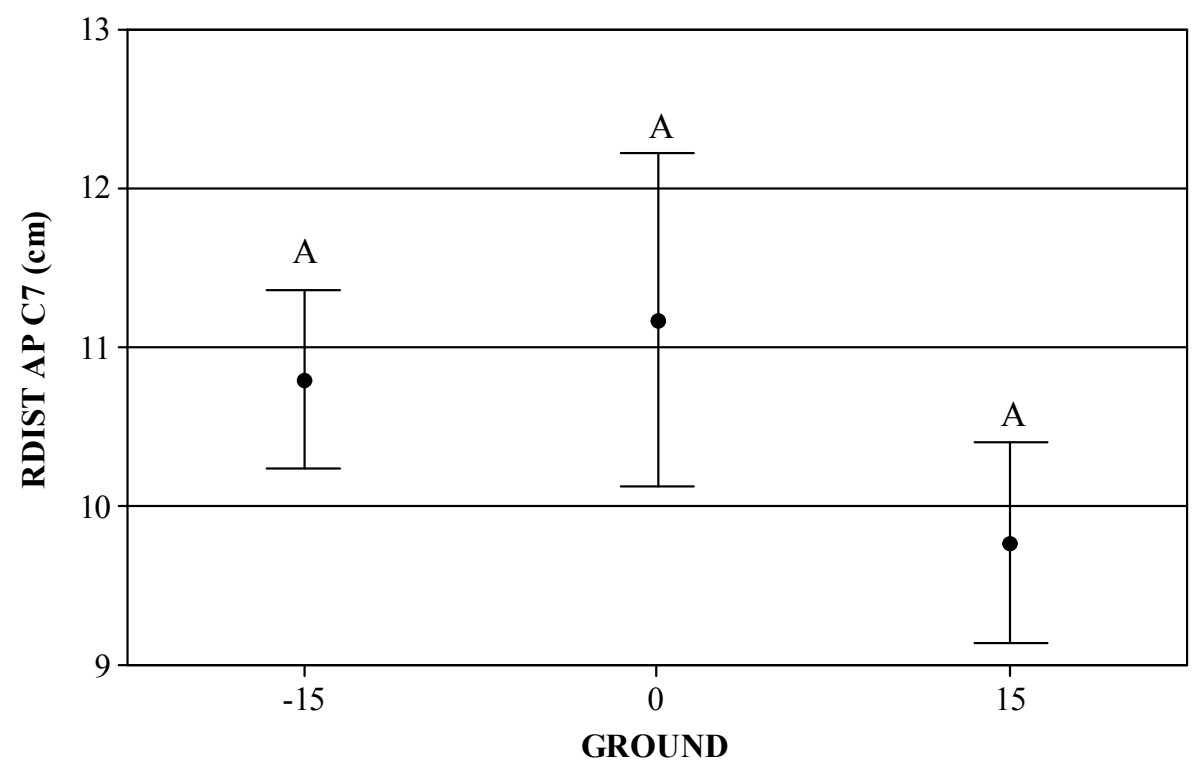

Figure 120: Effects of GROUND on C7 RDIST AP

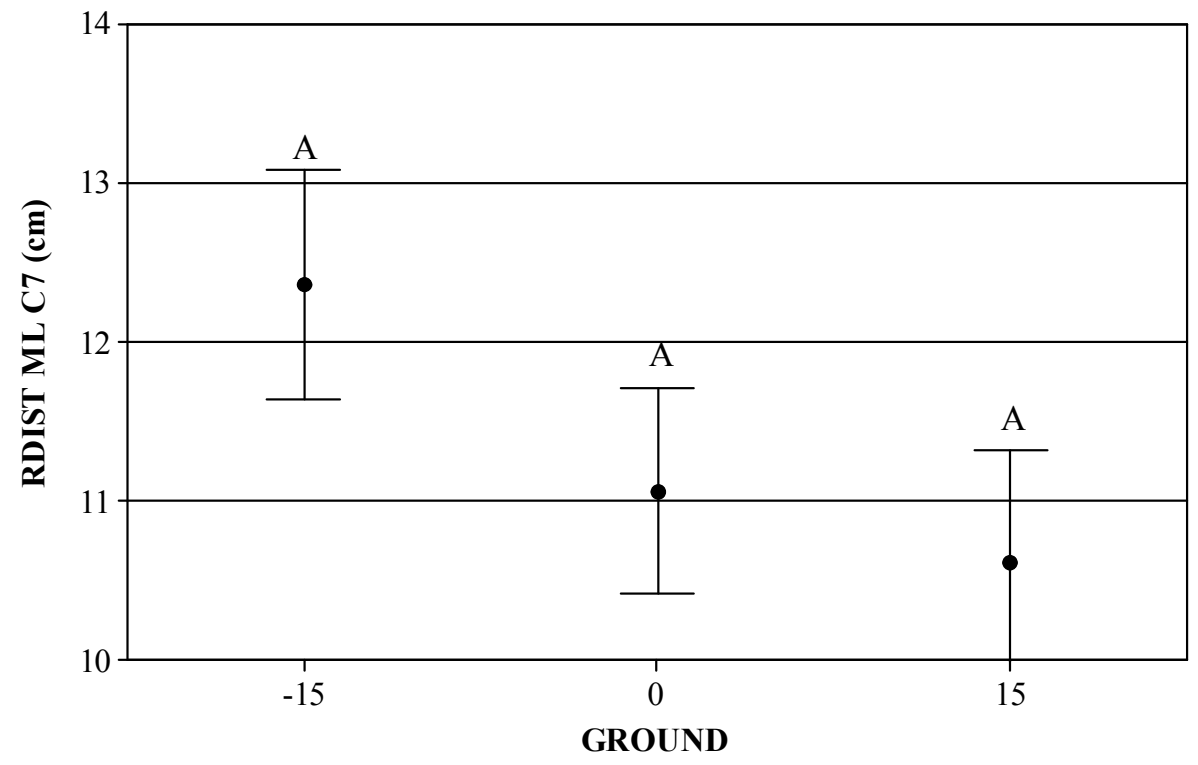

Figure 121: Effects of GROUND on C7 RDIST ML 


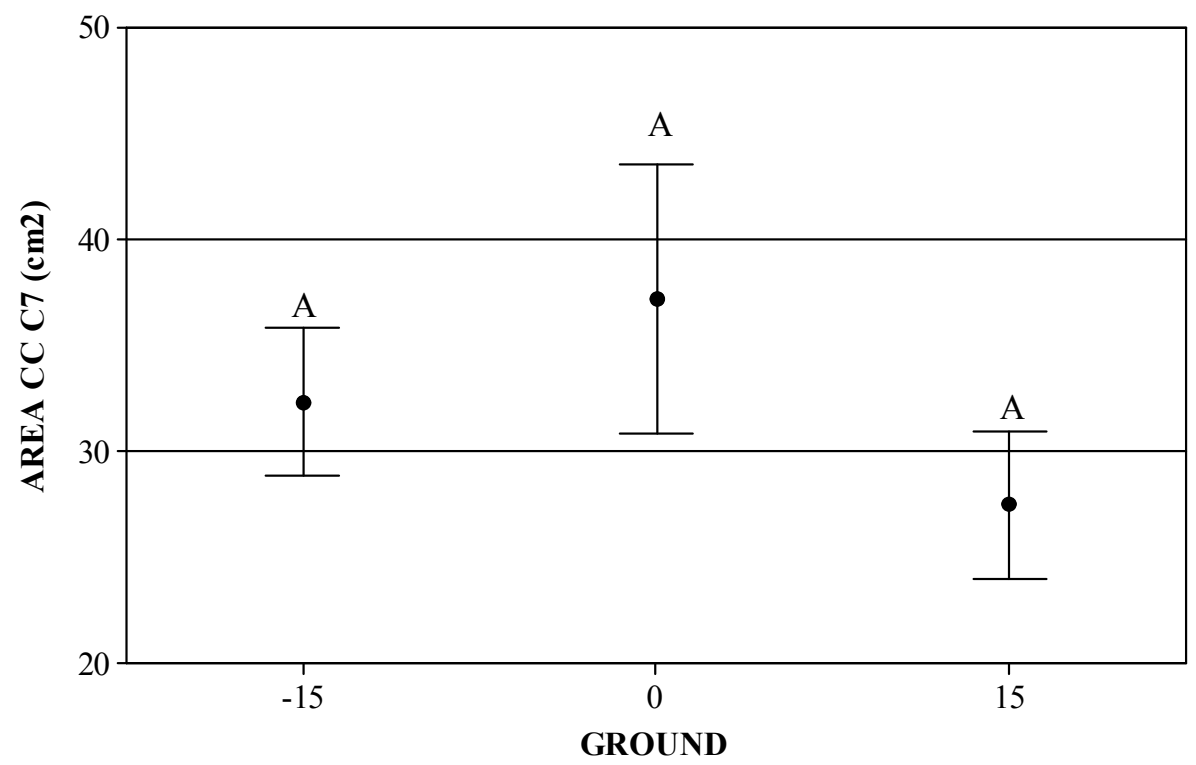

Figure 122: Effects of GROUND on C7 AREA CC

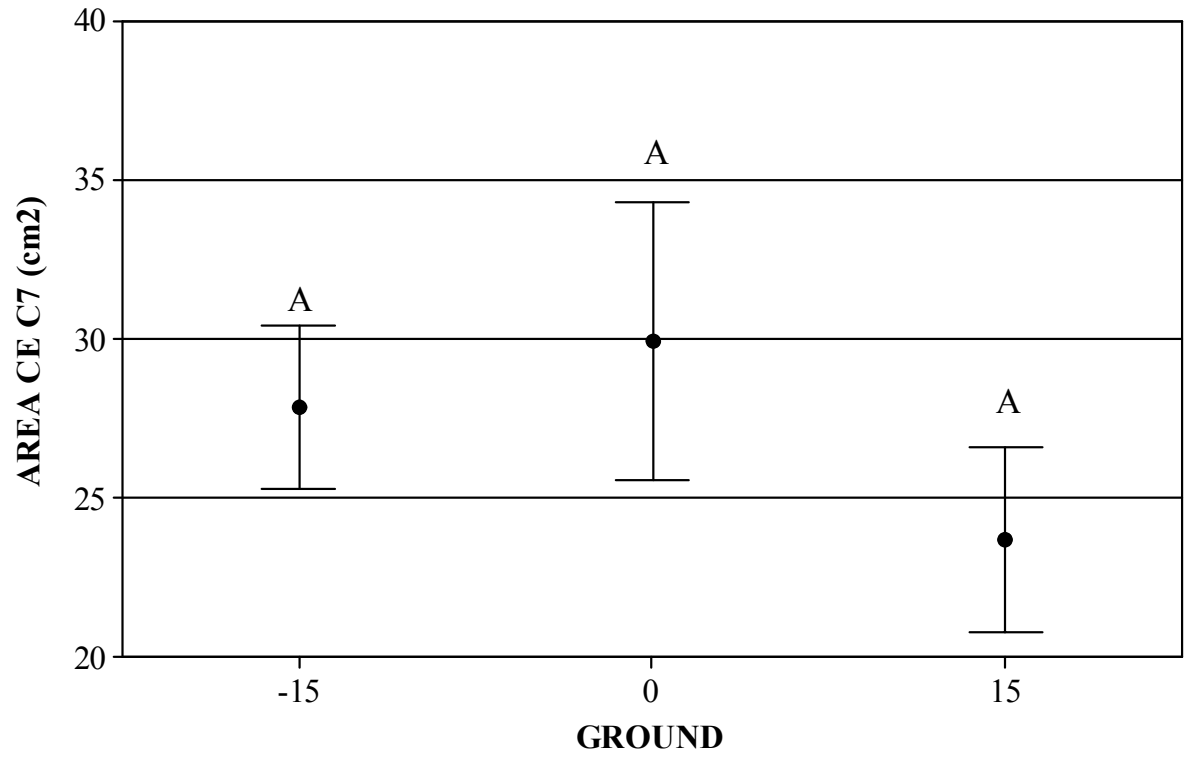

Figure 123: Effects of GROUND on C7 AREA CE 


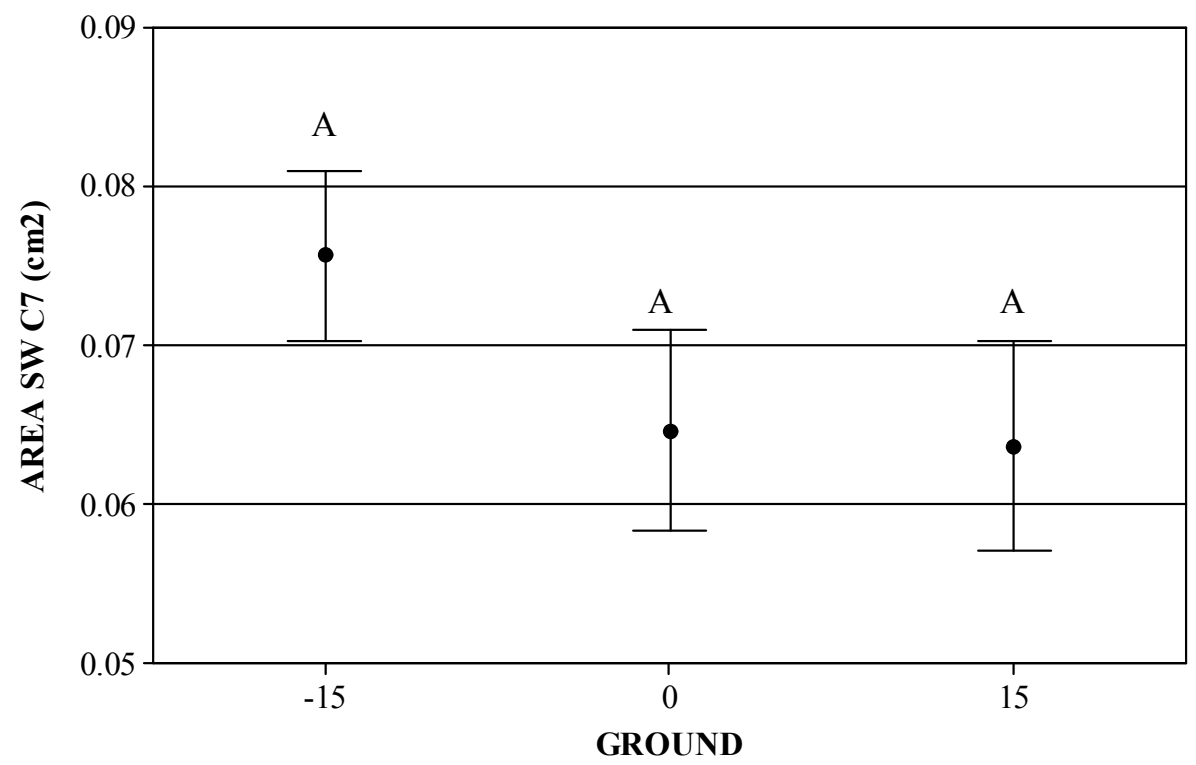

Figure 124: Effects of GROUND on C7 AREA SW

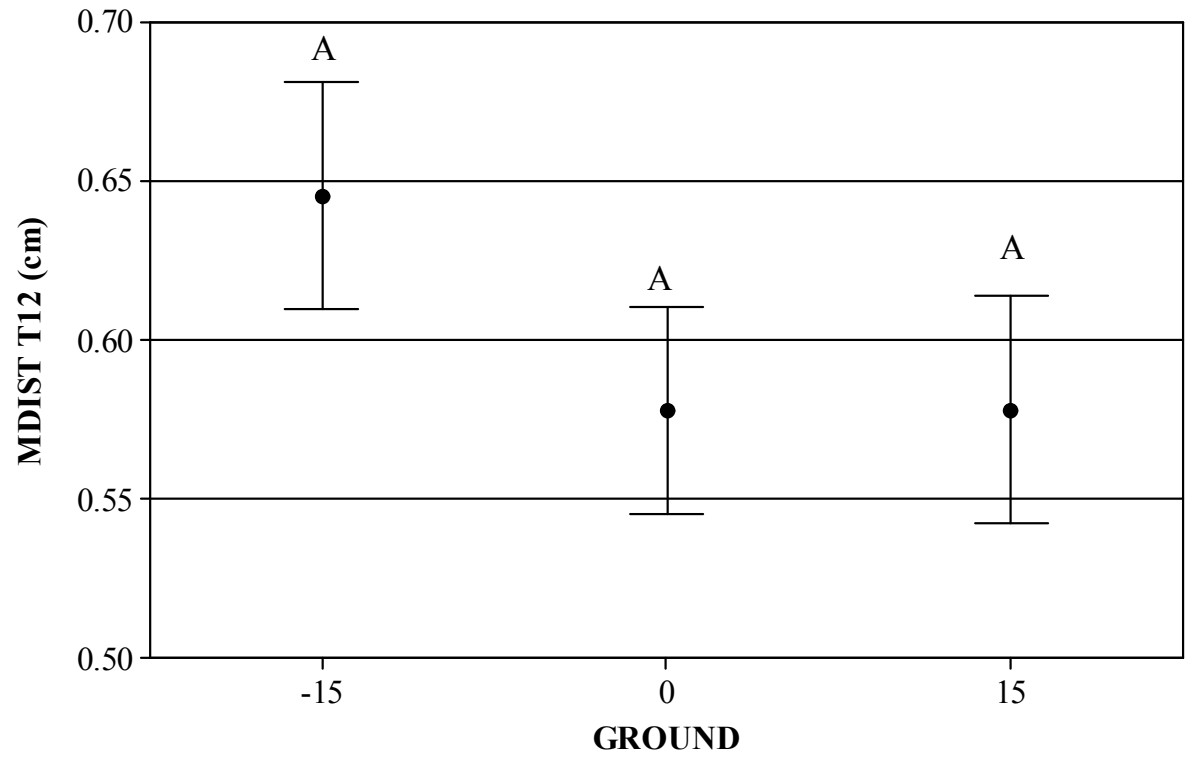

Figure 125: Effects of GROUND on T12 MDIST 


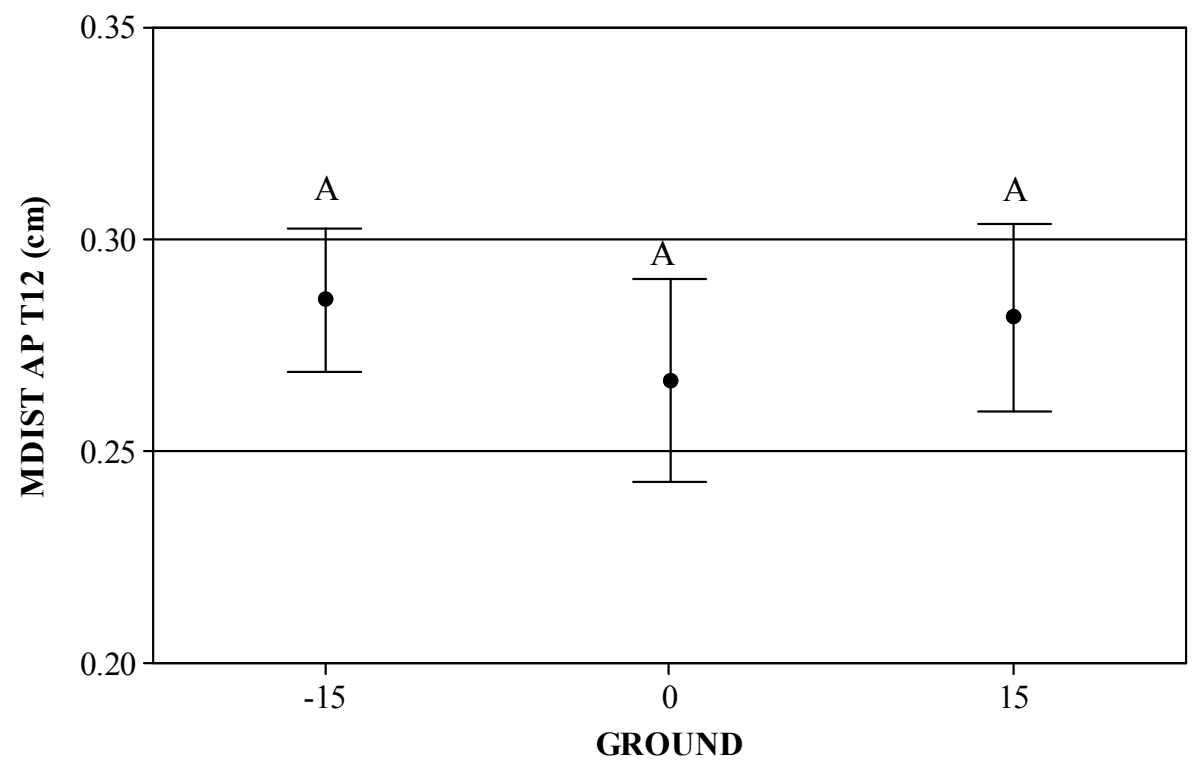

Figure 126: Effects of GROUND on T12 MDIST AP

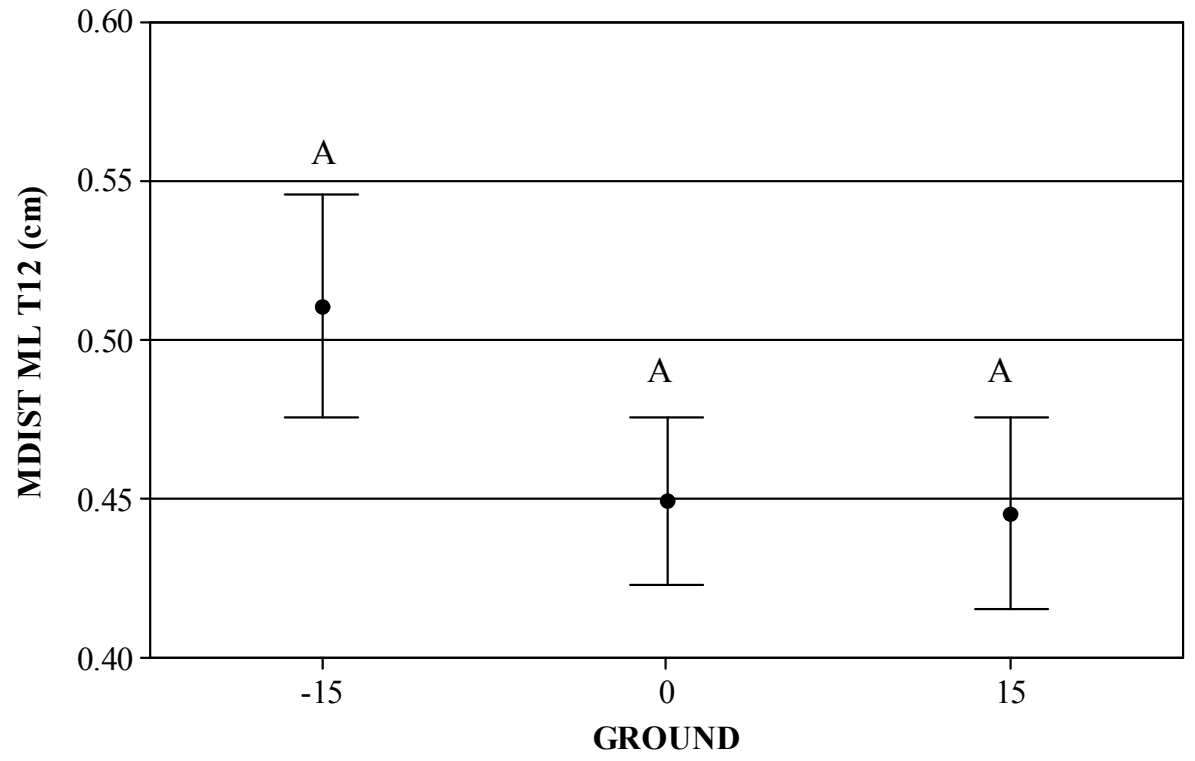

Figure 127: Effects of GROUND on T12 MDIST ML 


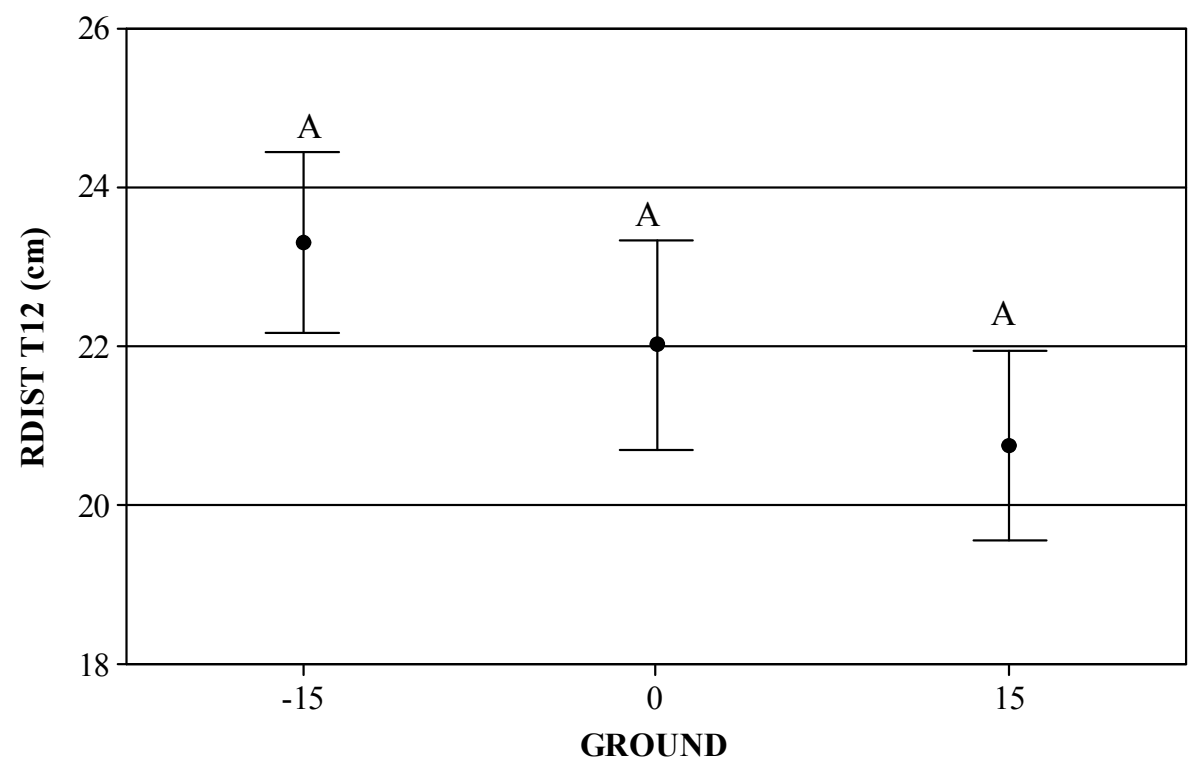

Figure 128: Effects of GROUND on T12 RDIST

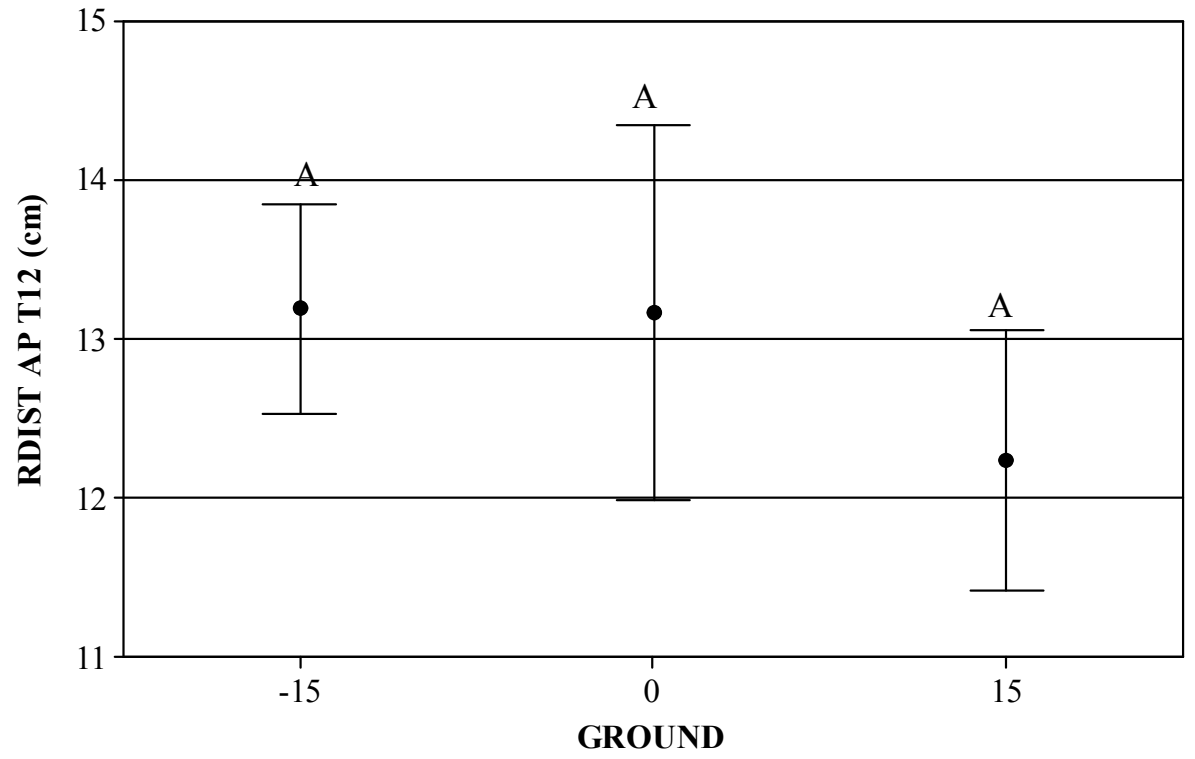

Figure 129: Effects of GROUND on T12 RDIST AP 


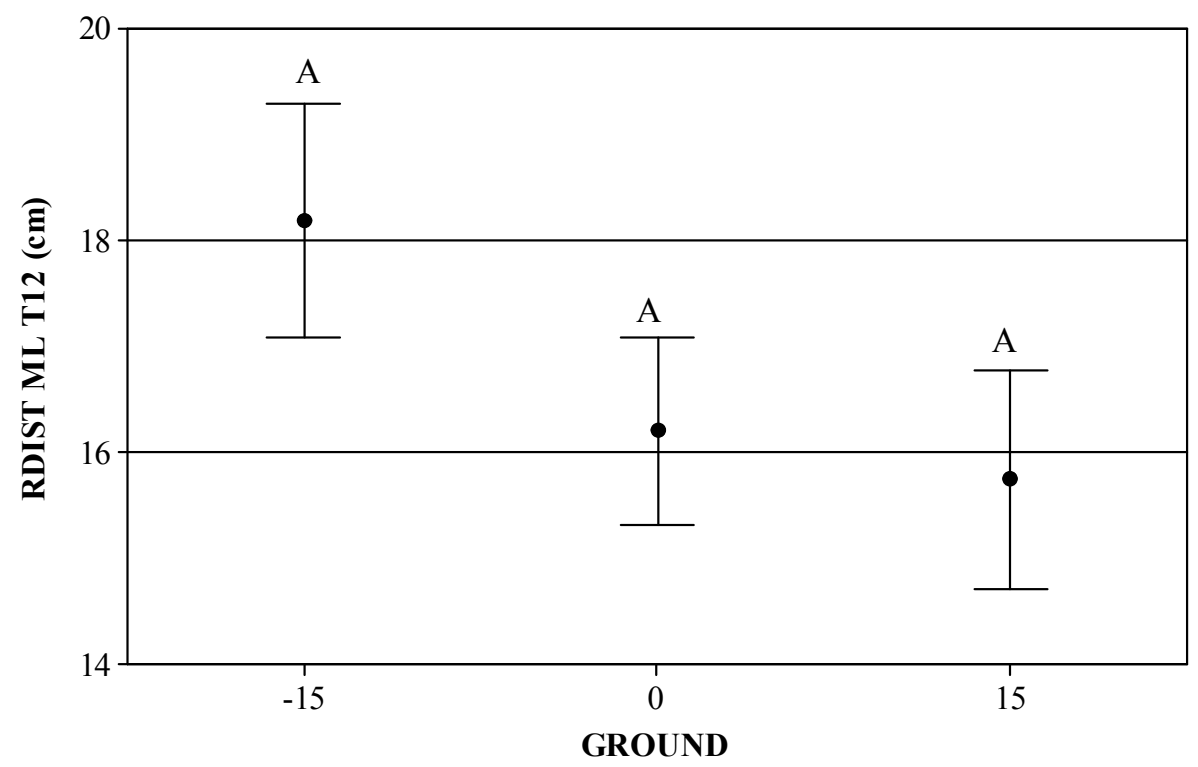

Figure 130: Effects of GROUND on T12 RDIST ML

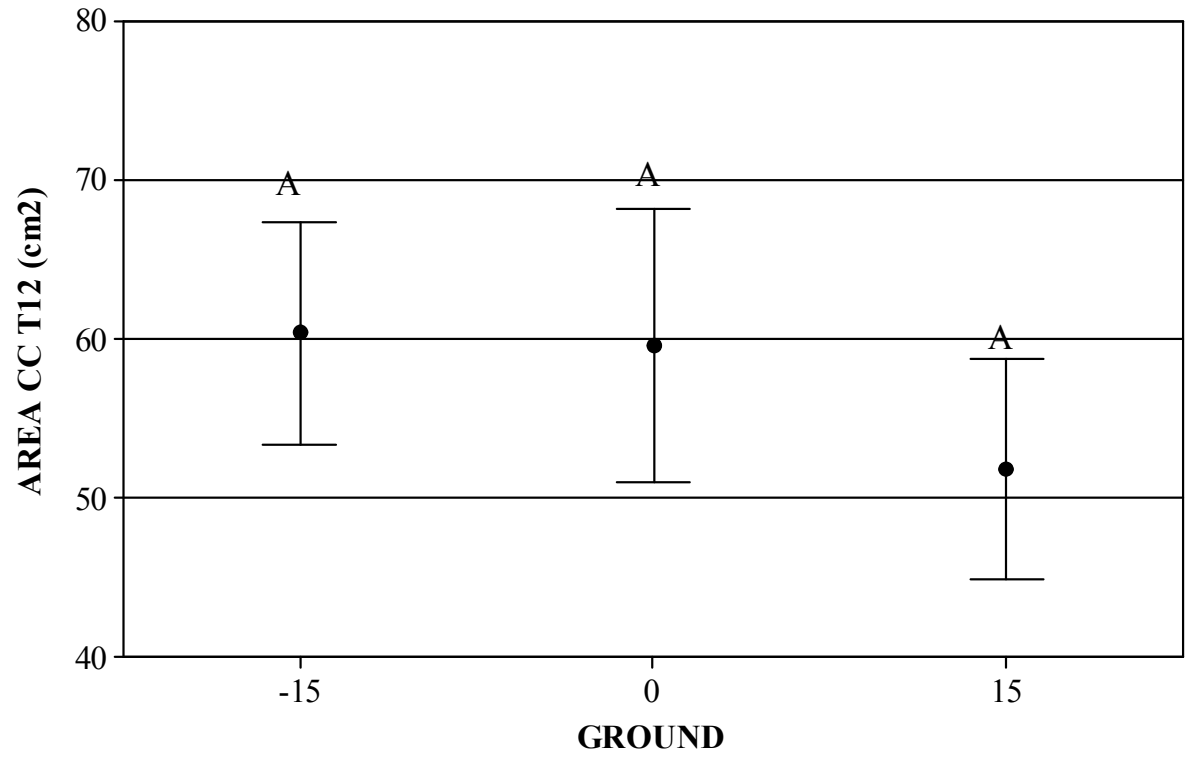

Figure 131: Effects of GROUND on T12 AREA CC 


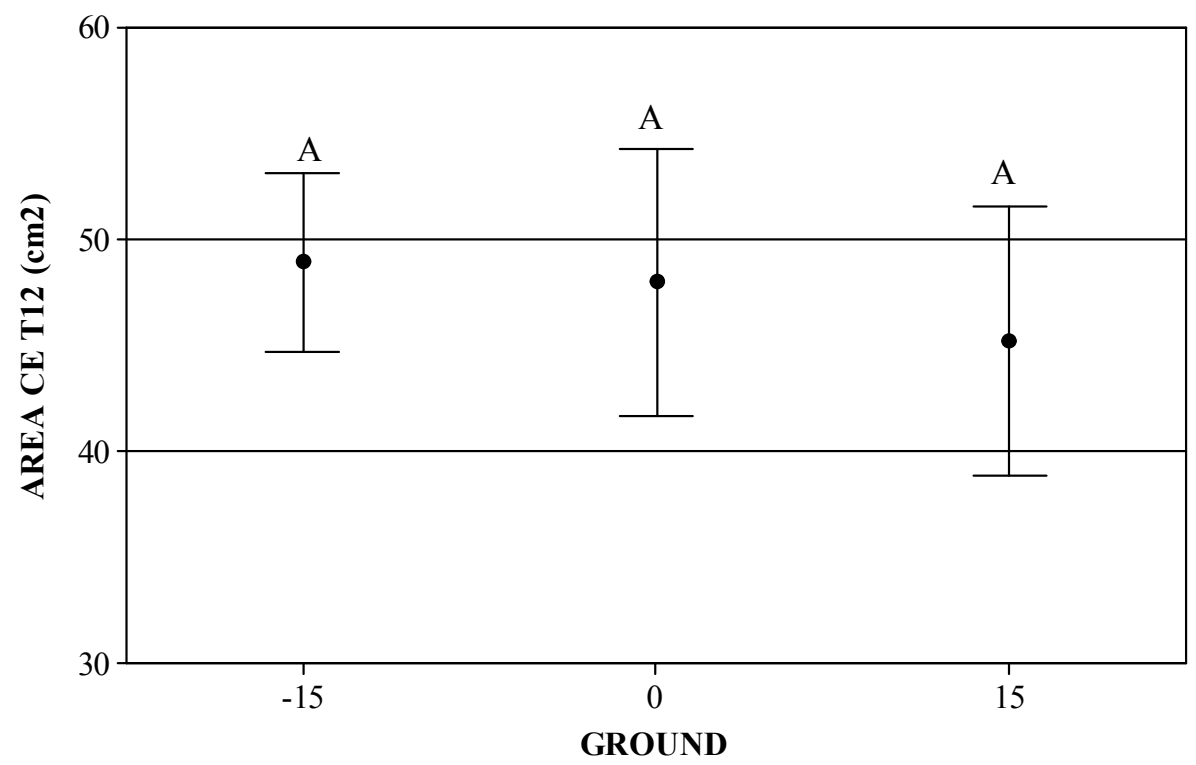

Figure 132: Effects of GROUND on T12 AREA CE

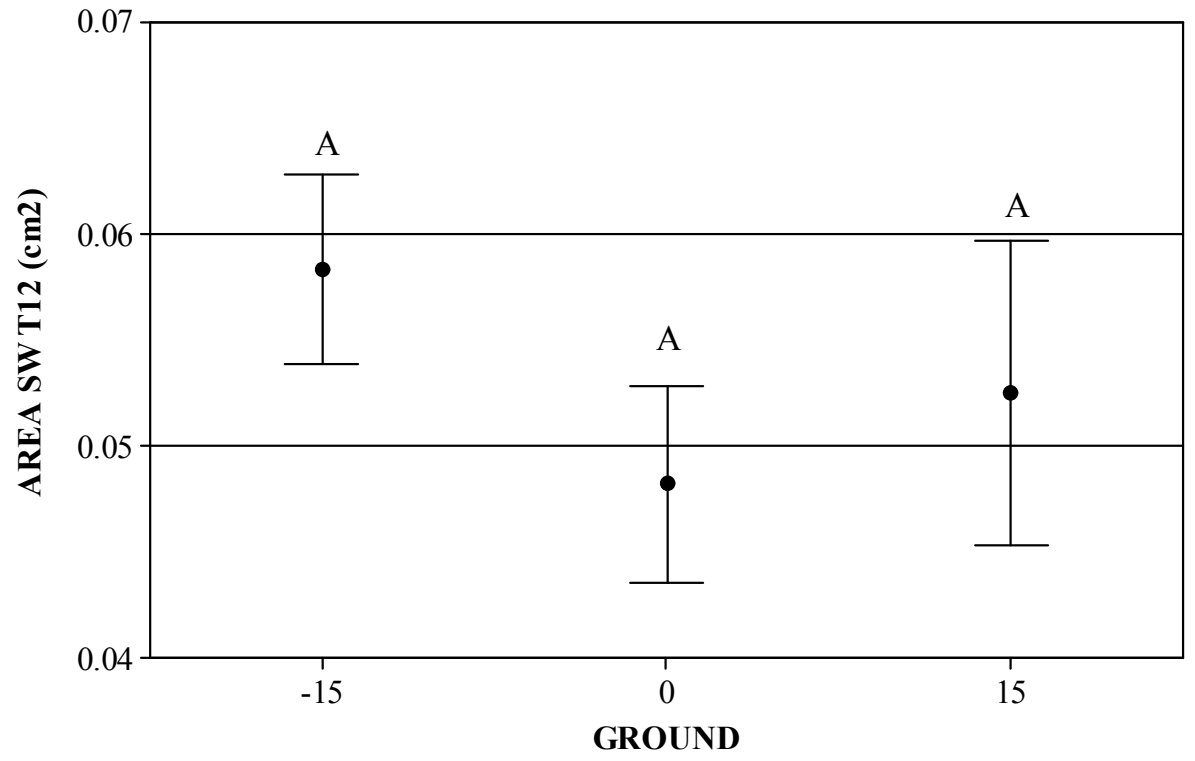

Figure 133: Effects of GROUND on T12 AREA SW 


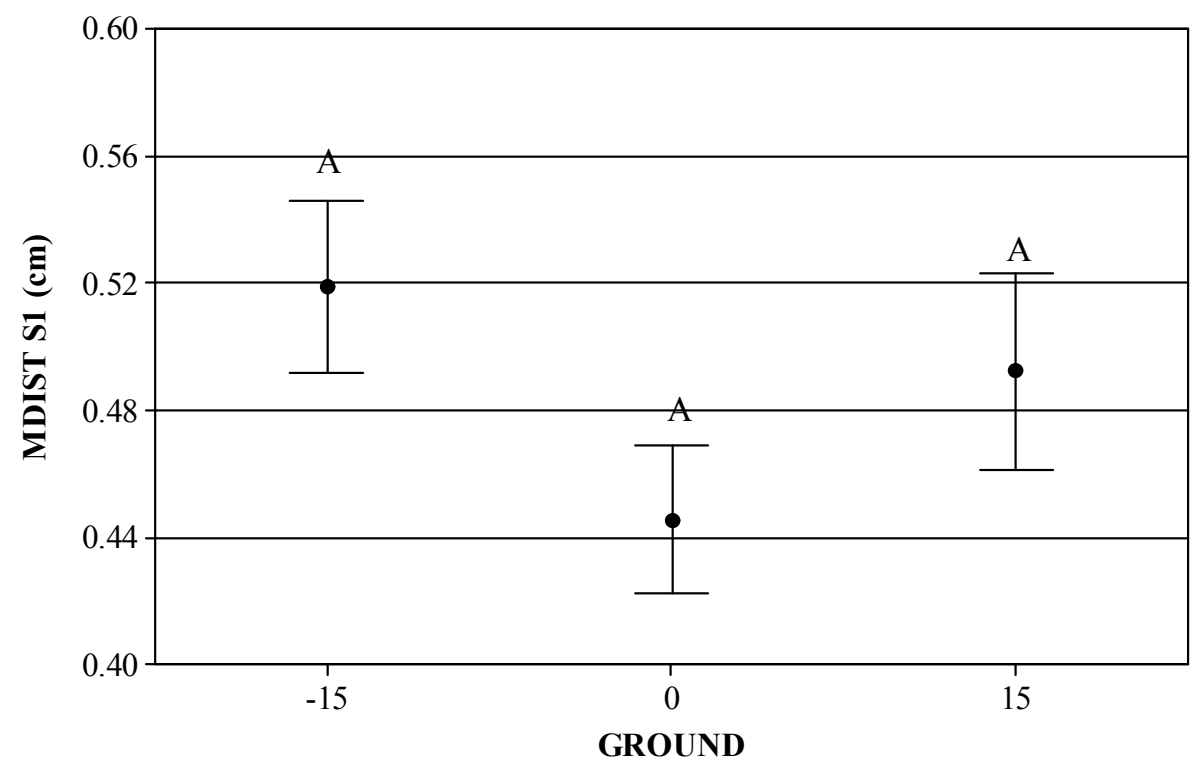

Figure 134: Effects of GROUND on S1 MDIST

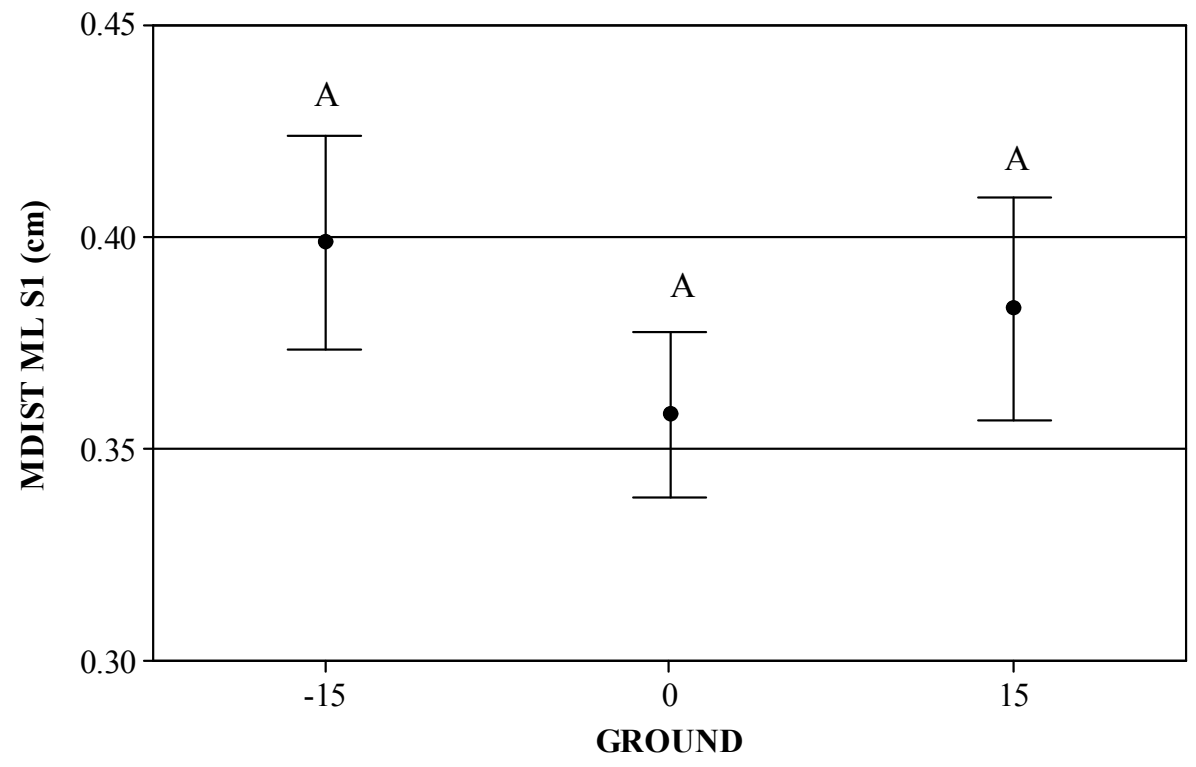

Figure 135: Effects of GROUND on S1 MDIST ML 


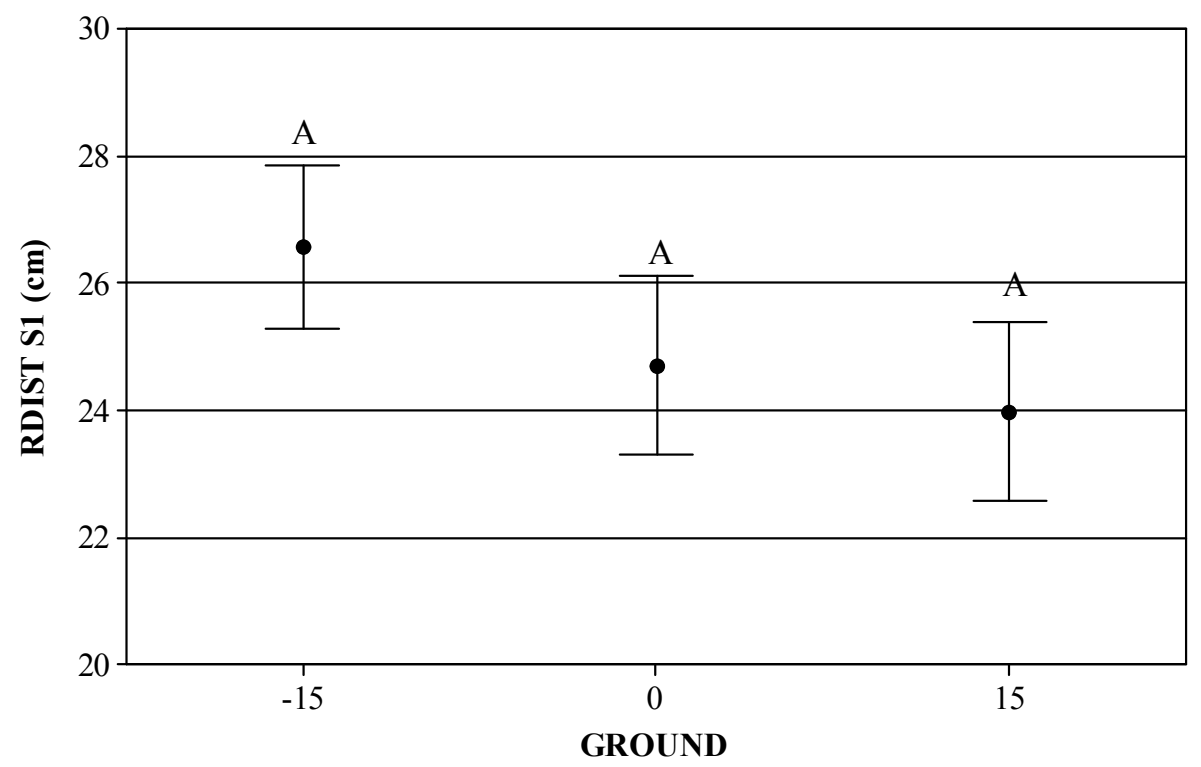

Figure 136: Effects of GROUND on S1 RDIST

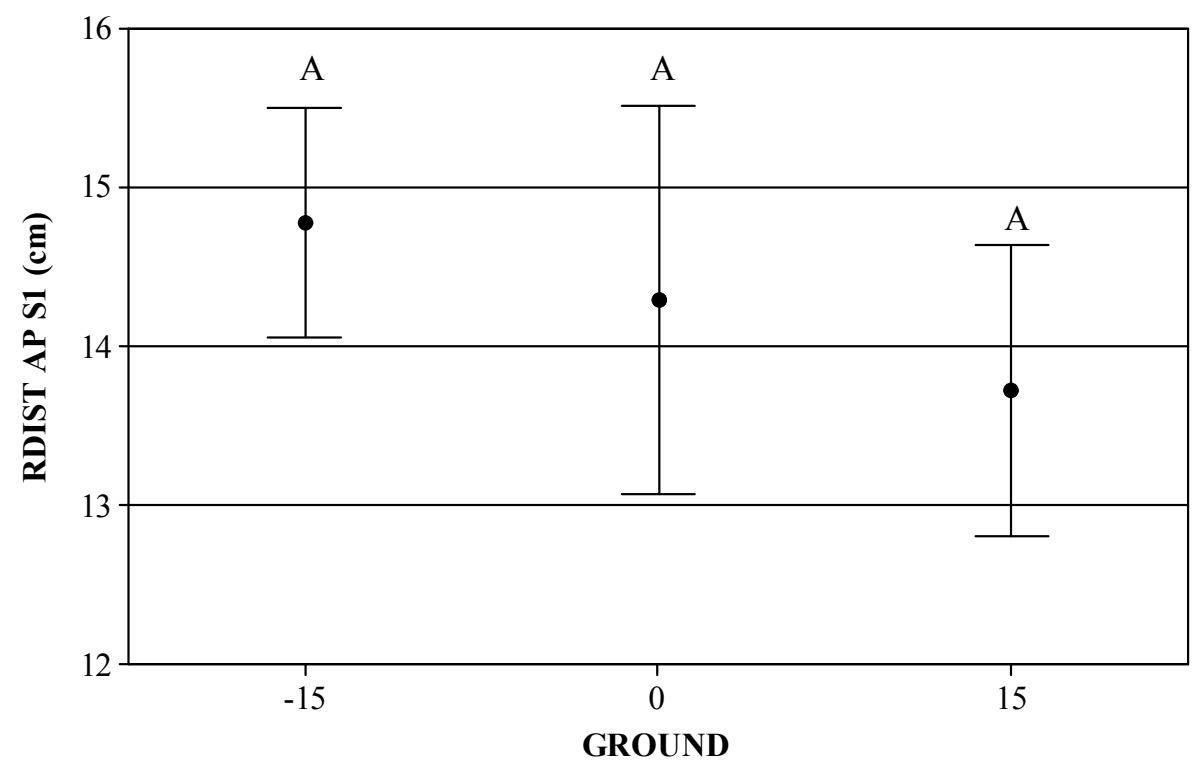

Figure 137: Effects of GROUND on S1 RDIST AP 


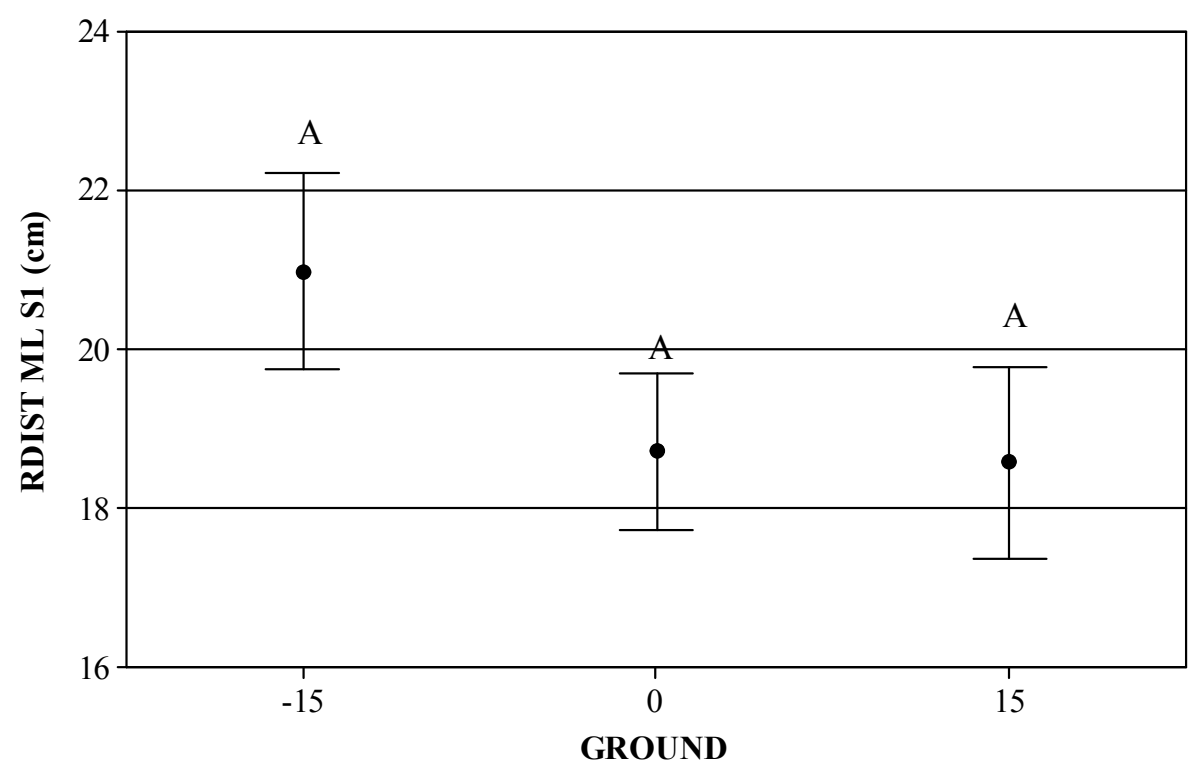

Figure 138: Effects of GROUND on S1 RDIST ML

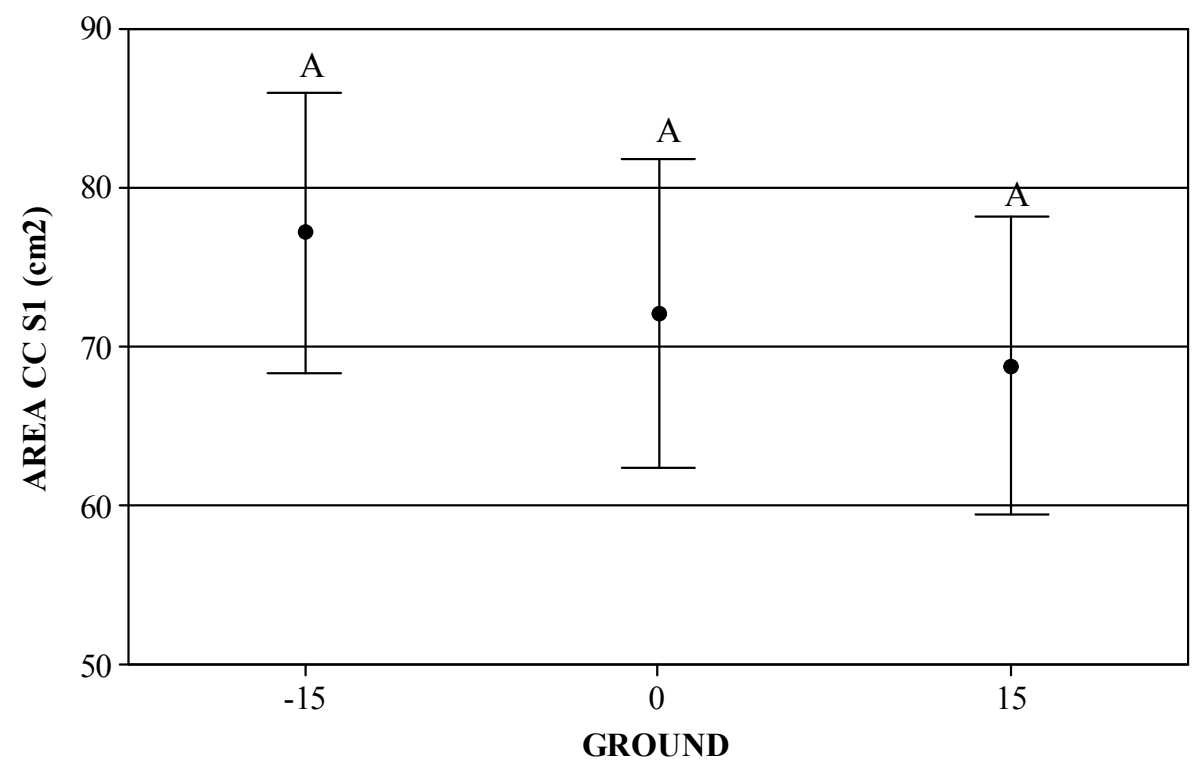

Figure 139: Effects of GROUND on S1 AREA CC 


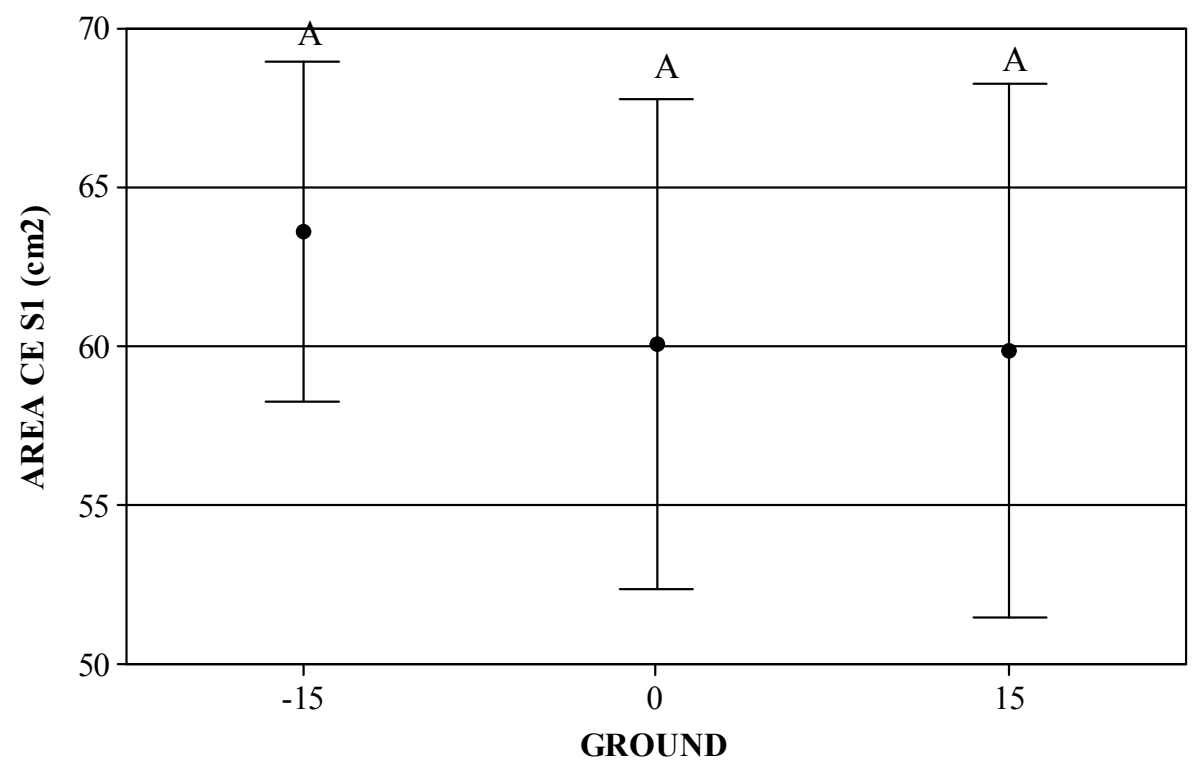

Figure 140: Effects of GROUND on S1 AREA CE 


\title{
APPENDIX D. INFORMED CONSENT DOCUMENT \\ W. WestVirginiaUniversity. \\ Office of Research Compliance \\ CONSENT AND INFORMATION FORM
}

\author{
OMR ICF
}

\section{Principal Investigator: Ning, Xiaopeng}

Department: ENGINEERING - Ind./Mgt. Sys. Engineering Tracking Number: $\mathrm{H}-$ 24367

\section{Study Title:}

The effect of uneven ground surface on the back muscle activation patterns during trunk flexionextension motion.

\section{Co-Investigator(s):}

Hu, Boyi, Zhou, Jie,

\section{Sponsor}

\section{Contact Persons}

In the event you experience any side effects or injury related to this research, you should contact Dr.Xiaopeng Ning at 304/294-9474. (After hours contact Dr.Xiaopeng Ning at 515/520-1951.) If you have any questions, concerns, or complaints about this research, you can contact Dr. Xiaopeng Ning at 304/294-9474

For information regarding your rights as a research subject, you may contact the Office of Research Compliance at 304/293-7073.

\section{Introduction}

In addition if you would like to discuss problems, concerns, have suggestions related to research, or would like to offer input about the research, contact the Office of Research Integrity and Compliance at 304293-7073.

You, , have been asked to participate in this research study, which has been explained to you by Boyi Hu. This study is being conducted by Xiaopeng Ning (PhD), Boyi Hu and Jie Zhou in the Department of Industrial and Management System Engineering at West Virginia University 


\section{Purposes of the Study}

The purpose of this study is to understand the low back muscle activation patterns during the performance of trunk bending tasks on slanted ground surface.

\section{Description of Procedures}

When experiment starts some measurement will be performed to measure your body dimensions such as your weight, height, trunk width. After that, 5 minutes warm up section will be provided to let you stretch and warm up the muscles of low back and other part of the body. You will then be fitted with a set of sensors designed to capture muscle activation levels (EMG) and 3mensional positions and angles (Motion sensors). EMG sensors will be placed over the skin of your abdominal and back muscles. Motion sensors will be place over the back of your neck and lower back region. You will then stand on an exercise device with your trunk; pelvis points and lower extremities secured and perform three trials of maximum lumbar extension tasks against a stationary resistance in a 20 degree of forward trunk flexion posture. Each maximum voluntary contraction trial will be five seconds long and a one minute rest period will be provided between exertions. Then you will be moved to the testing area and perform a total of 45 trunk bending tasks. You will be standing on two kinds of surfaces (slanted ground and stairs) to perform these tasks. Each trunk bending task will be completed in about 20 seconds. Between each trial, you will have a rest of 15 seconds. More rest will be provided if you feel tired. When finish the motion sensors and EMG surface electrodes will be removed and you will be free to go.

\section{Risks and Discomforts}

There is a risk for low back muscle strain and fatigue while performing the maximum exertions and other tasks. Therefore, you will be required to complete a warm up before these tasks and sufficient rest between trials.

\section{Alternatives}

You do not have to participate in this study.

The only alternative is to withdraw from this study.

\section{Benefits}

You may not receive any direct benefit from this study. The knowledge gained from this study may eventually benefit others. 


\section{Financial Considerations}

Subjects will not receive any compensation for participation in the study. It is very important for you to understand that neither the investigators nor WVU or its associated affiliations have the funds set aside to pay for the cost of lost work wages or any care or treatment that might be necessary because you get hurt or sick by taking part in this study. Any injuries that may result from this study would not be eligible for Workers'

Compensation as it will not be considered as work related injury. Understand that any treatments necessary will be billed to your health insurance, thus you may wish to consult your insurance provider before participating in this study.

\section{Confidentiality}

Any information about you that is obtained as a result of your participation in this research will be kept as confidential as legally possible. Your research records and test results, just like hospital records, may be subpoenaed by court order or may be inspected byfederal regulatory authorities without your additional consent. In any publications that result from this research, neither your name nor any information from which you might be identified will be published without your consent.

\section{Voluntary Participation}

Participation in this study is voluntary. You are free to withdraw your consent to participate in this study at any time. Refusal to participate or withdrawal will not affect your future care, [or your employee status at West Virginia University or your class standing or grades, as appropriate] and will involve no penalty to you. In the event new information becomes available that may affect your willingness to participate in this study, this information will be given to you so that you can make an informed decision about whether or not to continue your participation. You have been given the opportunity to ask questions about the research, and you have received answers concerning areas you did not understand.For WVU students, your involvement in or declination not to participate in the study will not impact your standing at WVU and/or your grades. 
Upon signing this form, you will receive a copy.

I willingly consent to participate in this research.

Signature of Subject or

Printed Name

Date

Time

Subjects Legal Representative

The participant has had the opportunity to have questions addressed. The participant willingly agrees to be in the study.

Signature of Investigator or

Printed Name

Date

Time

Co-Investigator 